\title{
Chern-Simons Theory with Vector Fermion Matter
}

\author{
Simone Giombi ${ }^{1, a}$, Shiraz Minwalla ${ }^{2, b}$, Shiroman Prakash ${ }^{2, c}$, \\ Sandip P. Trivedi ${ }^{2, d}$, Spenta R. Wadia ${ }^{2,3, e}$ and Xi Yin ${ }^{4, f}$ \\ ${ }^{1}$ Perimeter Institute for Theoretical Physics, Waterloo, Ontario, N2L 2Y5, Canada \\ ${ }^{2}$ Dept. of Theoretical Physics, Tata Institute of Fundamental Research, \\ Homi Bhabha Rd, Mumbai 400005, India. \\ ${ }^{3}$ International Centre for Theoretical Sciences, Tata Institute of Fundamental \\ Research, Homi Bhabha Rd, Mumbai 400005 and \\ TIFR Centre Building, Indian Institute of Science, Bangalore 560012, India \\ ${ }^{4}$ Center for the Fundamental Laws of Nature, Jefferson Physical Laboratory, \\ Harvard University, Cambridge, MA 02138 USA \\ ${ }^{a}$ sgiombi@pitp.ca, ${ }^{b}$ minwalla@theory.tifr.res.in, ${ }^{c}$ shiroman@gmail.com,

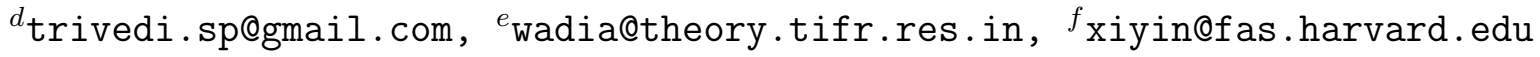

\begin{abstract}
We study three dimensional conformal field theories described by $U(N)$ ChernSimons theory at level $k$ coupled to massless fermions in the fundamental representation. By solving a Schwinger-Dyson equation in lightcone gauge, we compute the exact planar free energy of the theory at finite temperature on $\mathbb{R}^{2}$ as a function of the 't Hooft coupling $\lambda=N / k$. Employing a dimensional reduction regularization scheme, we find that the free energy vanishes at $|\lambda|=1$; the conformal theory does not exist for $|\lambda|>1$. We analyze the operator spectrum via the anomalous conservation relation for higher spin currents, and in particular show that the higher spin currents do not develop anomalous dimensions at leading order in $1 / N$. We present an integral equation whose solution in principle determines all correlators of these currents at leading order in $1 / N$ and present explicit perturbative results for all three point functions up to two loops. We also discuss a lightcone Hamiltonian formulation of this theory where a $W_{\infty}$ algebra arises. The maximally supersymmetric version of our theory is ABJ model with one gauge group taken to be $U(1)$, demonstrating that a pure higher spin gauge theory arises as a limit of string theory.
\end{abstract}




\section{Contents}

1 Introduction 4

1.1 Outline of the paper and summary of results . . . . . . . . 6 6

2 Free Energy on $\mathbb{R}^{2}$ at Finite Temperature $\quad 10$

2.1 Exact fermion propagator on $\mathbb{R}^{3} \ldots \ldots \ldots \ldots$

2.1.1 Exact solution of the gap equation . . . . . . . . . . 12

2.1.2 Rederivation of the gap equation as a Schwinger-Dyson equation 16

2.1.3 Rewriting the field theory as a path integral over singlet fields . 18

2.1.4 Diagrammatic expansion for the vacuum energy . . . . . . . 22

2.2 The finite temperature theory . . . . . . . . . . . . . . . 23

2.2.1 The finite temperature gap equation . . . . . . . . . . 24

2.2.2 The exact solution ................ 26 26

2.2.3 Free energy as a function of temperature . . . . . . . . 28

2.2.4 Explicit evaluation of the free energy . . . . . . . . . . . 29

2.3 Consistency of our gauge and regularization scheme . . . . . . . . . . 32

3 Operator Spectrum

3.1 The free limit . . . . . . . . . . . . . . . . . . 34

3.1.1 "Single trace" conformal representation content of the free theory 34

3.1.2 Explicit form of the primary operators . . . . . . . . . 35

3.1.3 The full multitrace partition function . . . . . . . . . . . 37

3.2 Non-renormalization of the scaling dimension of the current operators . 39

3.3 Explicit form of the current operators . . . . . . . . . . . . . . . 40

3.3.1 $J^{(3)}$ as an example ........................ 42

3.4 Anomalous dimensions of the current operators . . . . . . . . . . 43

3.5 Anomalous current conservation relations within correlation functions . 45

3.6 Non-renormalization of the scalar operator $J^{(0)}$. . . . . . . . . . . 46

4 Three Point Functions 47 
4.1 Quantum corrected operators and their 3-point functions . . . . . . 47

4.2 Three point functions in perturbation theory . . . . . . . . . . . 48

4.3 Three point functions in the free fermion theory . . . . . . . . . 49

4.4 A few comments on the general structure of three point functions . . . 51

4.5 One-loop and two-loop results . . . . . . . . . . . . . 54

5 The Formal Solution at Large $N$

5.1 Exact effective action for $\psi$ and $\Sigma \ldots \ldots . . \ldots \ldots$

5.2 Integral equation for the four Fermi vertex . . . . . . . . . . . 57

5.3 Effective action for $\psi$ fields alone . . . . . . . . . . . . 58

5.4 Exact evaluation of correlators . . . . . . . . . . . . . 59

6 Light-Cone Hamiltonian and $W_{\infty}$ Algebra $\quad 60$

6.1 Bilocal variables satisfying a $W_{\infty}$ algebra . . . . . . . . . . 63

6.1.1 Constraint on the bilocal fields . . . . . . . . . . . . 64

6.2 The path integral and the large- $N$ classical solution . . . . . . . . . 65

6.3 Large $N$ perturbation theory and a formal large $N$ solution . . . . . . . 67

7 Comments on the Holographic Dual 68

7.1 Vector models are dual to higher spin gauge theories . . . . . . . . 68

7.2 Parity violating Vasiliev theory . . . . . . . . . . . . 69

7.3 A higher spin gauge theory as a string theory . . . . . . . . . 72

8 Discussion $\quad 73$

A Conventions for Propagators and Gauge Conditions 78

A.1 Lightcone gauge . . . . . . . . . . . . . . . . . . 79

A.2 Temporal gauge . . . . . . . . . . . . . . . . 80

A.3 Feynman gauge ..................... 81

B Perturbative Analysis of Fermion Self Energy 81

B.1 Feynman gauge . . . . . . . . . . . . . . . . . 81 
B.2 Temporal gauge . . . . . . . . . . . . . . . . . . 82

B.3 Light cone gauge . . . . . . . . . . . . . . . . . . 83

C Lorentz invariance of the Wilson line at one loop 84

D Unitary Representations of the $d=3$ Conformal Group 86

E Primary Operators in Free and Interacting Fermion Theories 87

E.1 The generating function of conserved currents for free fermions . . . . . 87

E.2 Two-point functions of primary operators in the free theory . . . . . . 89

E.2.1 Simple examples . . . . . . . . . . . . . . . . . . . 90

E.2.2 Results for all spins . . . . . . . . . . . . . . 90

E.3 Explicit computation of the divergence of $J^{(3)} \ldots \ldots \ldots \ldots$

E.4 Higher spin examples . . . . . . . . . . . . . . . . . . . 93

F Perturbative Analysis of Anomalous Dimensions 94

F.1 Vanishing anomalous dimension of the scalar primary at planar level . . 94

F.2 Anomalous dimension of currents at order $1 / N \ldots \ldots \ldots \ldots$

F.3 Two-loop non-planar anomalous dimension of the scalar primary . . . 97

F.3.1 Feynman gauge . . . . . . . . . . . . . . . . . . 97

F.3.2 Temporal gauge . . . . . . . . . . . . . . . 97

F.3.3 Lightcone gauge . . . . . . . . . . . . . . . . . . . . . . 98

G Perturbative Analysis of Three Point Functions 100

G.1 Vanishing one-loop contribution to the three point functions of scalar operators . . . . . . . . . . . . . . . 100

G.1.1 Feynman gauge . . . . . . . . . . . . . . . . . . 101

G.1.2 Light cone gauge . . . . . . . . . . . . . . . . . 101

G.2 One-loop parity violating structure of $\langle J J J\rangle \ldots \ldots \ldots \ldots$

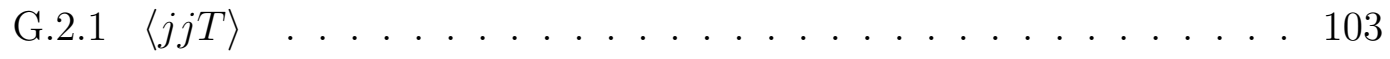

G.2.2 $\langle T T T\rangle \ldots \ldots \ldots \ldots \ldots \ldots \ldots$

G.3 Two-loop parity preserving structure of the three point functions . . . . 110 
G.3.1 $\langle T T T\rangle$ at two-loop . . . . . . . . . . . . . . . 110

G.3.2 General spins . . . . . . . . . . . . . . . . . . . . 114

\section{Introduction}

Chern-Simons theories are fascinating from several points of view. The attractive features of these theories, unique to three-dimensions, had been recognized early on, notably in [1] and [2]. They arise, for example, in the study of knot invariants [3], the classification of two-dimensional rational conformal field theories [4], and in the study of the quantum Hall effect [5]. More recently, superconformal Chern-Simons theories [6.7] have been shown to play a crucial role in the AdS/CFT correspondence [8].

In this paper we will be interested in non-supersymmetric, but conformally invariant, $U(N)$ Chern-Simons theories with matter. As we shall explain below, these theories have an important distinguishing feature; they effectively admit large $N$ nonsupersymmetric lines of fixed points of the renormalization group.

Lines of fixed points parameterized by a coupling constant are especially interesting from the viewpoint of the AdS/CFT correspondence. Such lines of $d$ dimensional CFTs have the potential of interpolating between a simple field theoretic description at weak coupling and a relatively simple bulk gravitational description at strong coupling, as demonstrated by the famous supersymmetric examples 9,8 .

Now when $d \geq 4$, lines of fixed points appear to be rather exotic. The examples we know of (like the large $N$ Banks-Zaks fixed line of QCD) involve theories with a parametrically large number of flavours of matter fields. It is interesting, on the other hand, that effective fixed lines of large $N$ Chern-Simons theories coupled to matter fields are plentiful and very easily constructed in $2+1$ dimensions even with very simple matter content!

Consider a level $k, U(N)$ Chern-Simons theory coupled to a single fermion in any representation of the gauge group. The only gauge invariant power counting relevant or marginal operators in such a theory are the fermion kinetic term and mass term. A continuum quantum theory built from such a Lagrangian depends on two discrete parameters, $k$ and $N$, and a single continuous parameter, the physical mass $m$ of the fermionic field. At energies $E \gg m$, the dynamics of this theory is scale invariant as well as nontrivial. Nontriviality is ensured by the fact that the discrete ChernSimons coupling, which induces interactions among the fermions, cannot run and so is nonvanishing even at arbitrarily high energy. The nontrivial CFT that controls the

\footnotetext{
${ }^{1}$ We thank O. Aharony for discussions on this point.
} 
high energy behaviour of this system is most directly constructed by choosing the bare mass to set the physical mass $m$ of this system to zero.

The parameters $k$ and $N$ labeling the CFT are discrete. However it is well known that the loop counting parameter in a $U(N)$ Chern-Simons theory is the 't Hooft coupling $\lambda=\frac{N}{k}$ whenever all matter fields transform in representations whose dimension does not grow faster with $N$ than $N^{2}$. In the large $N$ (and simultaneously large $k$ ) limit $\lambda$ is effectively a continuous parameter (exactly as in ABJM theory [8]). For this reason the discretum of CFTs described by integer values of $k$ and $N$ coalesces into a fixed line in the large $N$ limit.

We emphasize that a variety of such non-supersymmetric fixed lines exist in three dimensions. Choosing the fermions that transform in, say, the adjoint representation of $U(N)$ gives one such fixed line of theories. Choosing fermions that transform in the bifundamental of $U(N) \times U(M)$ yields another example - one that preserves parity when $N=M$ and the two Chern-Simons levels are equal and opposite 2 .

The study of fixed lines of large $N$ Chern-Simons theories with matter and their bulk duals appears to be an interesting programme. For examples with a large amount of supersymmetry this programme was spectacularly initiated by ABJM [8 and carried forward in several follow up papers $10-12$. In this paper we initiate a detailed study of perhaps the simplest of the non-supersymmetric fixed lines - the theory of a single fundamental fermion coupled to a $U(N)$ level $k$ Chern Simons theory turns out to be surprisingly tractable at all values of $\lambda$, for reasons we now explain.

The Chern-Simons coupled gauge field has $N^{2}$ components. Superficially, the large $N$ limit of these theories is governed by the summation over a complicated web of planar graphs. The complexity is illusory, as pure Chern-Simons theory has no propagating degrees of freedom - the only propagating degrees of freedom in our system are the fundamental fermions. Consequently, the theory we investigate is a vector model with $N$ degrees of freedom. Large $N$ limits of vector theories are much simpler than their matrix counterparts, and sometimes prove to be exactly solvable. Indeed, both in terms of diagrammatics and canonical structure, the theory we study bares a close resemblance to 't Hooft's solution of two-dimensional QCD in the large- $N$ limit using light-cone gauge 13$]]^{3}$

The simplicity of the vector model in the large $N$ limit, combined with the crucial choice of light-cone gauge, allows us to derive several exact results (valid to leading

\footnotetext{
${ }^{2}$ The ease of construction of conformal field theories in $d=3$ intriguingly suggests that it is particularly easy to construct non supersymmetric quantum theories of gravity in $d=4$.

${ }^{3}$ It has been conjectured recently 14 that the appropriate two dimensional analogs of large $N$ vector models are $W_{N}$ minimal models. Its duality with higher spin gauge theories and in particular Vasiliev's system in $A d S_{3}$ are further explored in $15,19$.
} 
order in $N$ but to all orders in $\lambda$.) One of our key results is the exact expression for the free energy of our theory as a function of $\lambda$ and temperature. In obtaining this result we find that our line of fixed points exists only if $0 \leq \lambda<1$. We also enumerate the spectrum of "single trace" primary operators and demonstrate that their scaling dimensions are not renormalized as a function of $\lambda$. We present an integral equation whose solution in principle determines all correlation functions of all these non renormalized operators - though we leave a detailed study of this integral equation and its solutions for future work. Based on the analysis of the spectrum of primary operators and explicit computation of some three point functions at low orders in perturbation theory, we also make several statements about the as-yet-unidentified holographic dual to our theory, which, as we discuss, must be a higher spin gauge theory. In the rest of this introduction we will summarize the key results of our paper in more detail.

\subsection{Outline of the paper and summary of results}

A summary of our main results is as follows.

In section 2, we first calculate the exact fermion propagator for the theory on $\mathbb{R}^{3}$ in light-cone gauge. Our result, which is valid to leading order in the $\frac{1}{N}$ expansion but is exact in $\lambda$ is listed in (2.23). We then turn to the finite temperature partition function of our theory, i.e., the partition function of the theory on $\mathbb{R}^{2} \times S^{1}$. We demonstrate that this partition function is completely determined by the self energy in the exact fermion propagator on $\mathbb{R}^{2} \times S^{1}$. This self energy obeys a nonlinear Schwinger-Dyson integral equation (2.66), which, by an apparent accident of numbers, turns out to admit a remarkably simple exact solution (2.80). Using this solution, we find that the free energy of our theory as a function of temperature and $\lambda$, in a box of volume $V_{2}$ (which is taken to be very large) is given by

$$
F=-\frac{N V_{2} T^{3}}{6 \pi}\left[\tilde{c}^{3} \frac{1-\lambda}{\lambda}+6 \int_{\tilde{c}}^{\infty} d y y \ln \left(1+e^{-y}\right)\right],
$$

where $\tilde{c}$ is the unique real solution to the equation $\bigsqcup^{4}$

$$
\tilde{c}=2 \lambda \ln \left(2 \cosh \frac{\tilde{c}}{2}\right) .
$$

(1.2) has no real solutions for $|\lambda|>1$; indeed, our fixed line of theories exists only in the interval $|\lambda| \in[0,1)$.

The fact that the free energy is proportional to $-N V_{2} T^{3}$. is an immediate consequence of the extensivity, conformality and large $N$ counting (disc diagrams dominate

\footnotetext{
${ }^{4}$ Though it is not manifest the free energy in eq.1.1) is an even function of $\lambda$, as is required by the invariance of the free energy under parity.
} 
the free energy at large $N$ ), and could have been asserted on general grounds. The nontrivial part of $(1.1)$ is the function of $\lambda$,

$$
h(\lambda)=\frac{1}{6 \pi}\left[\tilde{c}^{3} \frac{1-\lambda}{\lambda}+6 \int_{\tilde{c}}^{\infty} d y y \ln \left(1+e^{-y}\right)\right],
$$

that multiplies the factor $-V_{2} T^{3} N$. About $\lambda=0$ this function has an analytic expansion in even powers of $\lambda$. As $|\lambda|$ increases in $[0,1)$, this function decreases monotonically from the free value $\frac{3}{4 \pi} \zeta(3)$ to zero (see Fig 1 in section 2 ). $h(\lambda)$ is a measure of the number of available states in our system; the fact that it monotonically decreases implies a thinning of degrees of freedom at stronger coupling that is taken to an extreme as $\lambda \rightarrow 1$, when $h(\lambda)$ vanishes. Recall that the ABJM theory exhibits a similar phenomenon as $\lambda$ is scaled to $\infty$.

In the strict large $N$ limit we expect all transport properties of the finite temperature phase to be governed by a collision-less Boltzmann transport equation (the analogue of equation 9.66 of [22] in the critical $O(N)$ sigma model in 3 dimensions, see Section 85 5 . In other words the finite temperature system appears to behave like a collection of free fermions with a $\lambda$ and temperature dependent dispersion relation. This conclusion is also suggested by the fact that the exact finite temperature fermion two point function 2.80 has an extremely simple analytic structure as a function the Lorentzian frequency $\omega$. The propagator has no cuts on the real $\omega$ axis The only singularity in this propagator in the complex $\omega$ plane, are the poles located at

$$
\omega= \pm \sqrt{k^{2}+\tilde{c}^{2} T^{2}}
$$

In section 3, we study the spectrum of those operators whose dimension stays fixed in the large $N$ limit. All such operators are constructed as a product of "single trace" operators, where by "single trace" we mean operators such as $\bar{\psi}^{i} \psi_{i}$, which are formed out of the contraction of a single fundamental fermionic index with a single antifundamental fermionic index. We demonstrate that the spectrum of single trace operators is not renormalized as a function of $\lambda$ at leading order in $N$. The set of single trace primaries is given, as in the free theory [28, by a single "current" $J_{\mu_{1} \ldots \mu_{s}}^{(s)}$ of dimension $s+1$ at every spin $s=1,2, \ldots, \infty$ together with the single dimension two scalar $\bar{\psi} \psi$.

In the strict large $N$ limit, all the operators listed above except for the scalar are primaries of short representations of the conformal algebra as we now explain. Short representations always have null states: the null states for a dimension $s+1$, spin $s$ current are simply the states formed out of the divergence of the current. While the

\footnotetext{
${ }^{5}$ We thank K. Damle and S. Dutta for discussions on this point.
} 
divergence of $J_{\mu_{1} \ldots \mu_{s}}^{(s)}$ does vanish in the free theory, at nonzero coupling the currents obey an equation of the schematic form

$$
\partial \cdot J^{(s)} \sim \frac{1}{k} J J+\frac{1}{k^{2}} J J J
$$

Although the RHS of $(1.3)$ is nonvanishing, it is a multitrace contribution, and so contributes only at subleading order in $\frac{1}{N}$ when inserted into a two point function. In other words the currents $J^{(s)}$ are effectively conserved, hence protected, within two point functions, at leading order in large $N$. The operators $J^{(s)}$ develop anomalous dimensions at first subleading order in $\frac{1}{N}$ (see subsection 3.4 for details).

A complete solution of our theory requires an algorithm to compute all correlation functions of $J^{(s)}$ at every value of $\lambda$. In section 4 we demonstrate that the three point functions of the spin operators, with all free indices chosen to lie in the $x^{-}$direction ${ }^{6}$, are determined once we solve the integral equation (4.4) that determines "quantum corrected" versions of the corresponding operators. In section 5 we also derive the integral equation (5.2) whose solution determines the full quantum effective action of the theory in terms of the fermionic fields. This effective action may be used to compute arbitrary correlators of the spin operators with all polarizations in the $x^{-}$direction. We leave a study of the relevant integral equations, and of the correlation functions of currents with arbitrary polarizations, to future work. In this paper (see section 4) we have contented ourselves with a study of three point functions of the current operators at tree, one-loop and two-loop level obtained using perturbative techniques. In particular, we demonstrate that certain parity odd tensor structures of three point functions recently found in 35,36 arise at one-loop order.

In section 6, motivated by the natural appearance of bilocal variables in the large$N$ limit (see, e.g., [46]), we briefly discuss a light-cone Hamiltonian formulation that enables one to express the theory entirely in terms of bilocal variables, $M(x, y)$, that satisfy a $W_{\infty}$ algebra. Using the method of coadjoint orbits, we then derive an action for these bilocal variables and study its large- $N$ saddle point, which turns out to be particularly simple.

What is the bulk dual description of the line of CFTs studied in this paper? In order to address this question, we first note that it has been conjectured in [27], extending [25] (see also 26 for earlier related work), that our field theory in the free $\lambda \rightarrow 0$ limit admits a dual description as the parity-preserving "type-B" Vasiliev theory [24]. We will assume the correctness of this conjecture in what follows. Clearly, then, the bulk description of our finite $\lambda$ theory is some deformation of the type B Vasiliev theory. It is also clear that deformation must continue to preserve higher spin symmetry in the bulk

\footnotetext{
${ }^{6} x^{-}$refers to a null direction, and we are working in the light-cone gauge $A_{-}=0$.
} 
at the classical level; any bulk deformation that explicitly breaks Vasiliev's higher spin symmetry classically would require the introduction of new bulk fields (representing the longitudinal polarizations). The fact that the spectrum of single trace operators in our theory is not renormalized as a function of $\lambda$ implies that the dual theory does not have new fields corresponding to longitudinal polarizations, and so presumably does not break the higher spin invariance classically. It follows that the dual description is some higher spin theory in $A d S_{4}$ at every value of $\lambda$, in which higher spin symmetry is broken at loop level in the bulk.

Vasiliev has written down a family of higher spin theories in $A d S_{4}$, parameterized by a single function of one variable [24]. The cubic interactions in this family of theories, for instance, are governed by a phase (as well as the overall coupling constant). The parity preserving type $\mathrm{B}$ theory (dual to the parity preserving free fermion theory) is a special one in this family, where the phase is equal to $\pi / 2$ in some conventions, see e.g. 27. The only other parity preserving theory, known as type A model, is such that the phase vanishes, and is conjecturally dual to the free/critical bosonic vector model [25]. Theories with generic phase different from 0 or $\pi / 2$ do not preserve parity. It is natural to suggest that the dual description of the line of theories studied in this paper is given by a one parameter (the parameter is $\lambda$ ) set of such generalized Vasiliev theories with a $\lambda$ dependent phase. However, the evidence for this suggestion appears mixed (see section 7). A preliminary bulk study of 3 point functions of operators computed using the phase generalized Vasiliev theory, suggests that the result should be given by the schematic form

$$
\sin ^{2} \theta\langle J J J\rangle_{F}+\cos ^{2} \theta\langle J J J\rangle_{B}
$$

where $\langle J J J\rangle_{F}$ is the current-current-current 3 point function in the large $\mathrm{N}$ free Fermi theory and $\langle J J J\rangle_{B}$ is the corresponding 3 point function in the large $\mathrm{N}$ free boson theory. As we describe in section 4 below, the explicit perturbative 3 point functions described in the paragraph above appear to agree impressively with this prediction at two loops if we set $\theta=\frac{\pi}{2}(1-\lambda)+\mathcal{O}\left(\lambda^{3}\right) \cdot t^{7}$ On the other hand this identification, together with (1.4), implies that all one loop contributions to current-current-current three point functions vanish. As far as we can tell this is not the case. Conformal invariance allows parity violating structures for the three point functions of currents [36], and these structure appear to be generated, in field theory, with nonzero coefficient at order $\lambda$ (see section 4). We are not sure what to make of this disagreement. If we accept it

\footnotetext{
${ }^{7}$ One may speculate that this relation between $\theta$ and $\lambda$ is in fact exact, and that our theory in the $\lambda \rightarrow 1$ limit could be a theory of weakly coupled bosons! There is an analog in two dimensions: the $W_{N}$ minimal model 14 has a 't Hooft coupling $\lambda$, which also ranges from 0 to 1 . In the $\lambda \rightarrow 0$ limit it becomes a theory of free fermions, while in the $\lambda \rightarrow 1$ limit it becomes a theory of free bosons (modulo subtleties with twisted sectors).
} 
in the most straightforward way, it would suggest that our theory is not dual to the general phase Vasiliev model and that our line of Chern-Simons theory is dual to a yet-to-be-constructed higher spin theory that reduces, in the $\lambda \rightarrow 0$ limit, to the parity preserving Vasiliev theory. It would be especially interesting to identify the bulk dual to our field theory in the strong coupling limit $\lambda \rightarrow 1$. However we leave the resolution of these issues (and several others) to future work.

In subsection 7.3 we also note that the maximally supersymmetric extension of the theory studied in this paper is precisely the ABJ model [12] with one of the gauge groups taken to be $U(1)$. It follows that the higher spin bulk dual to the maximally supersymmetric version of the model studied in this paper is a particular limit of string theory.

In summary, in this paper we have initiated the task of determining an exact solution to a simple but nontrivial fixed line of quantum field theories, namely $U(N)$ Chern-Simons theories coupled to fermionic fundamental matter. We have been able to exactly compute the finite temperature partition function. It is possible that correlation functions in this theory are also exactly computable both at zero and at finite temperature. Exact results for current-current and stress tensor-stress tensor two point functions, as functions of temperature, would be particularly fascinating to obtain. Our work also suggests several obvious generalizations, for instance to the theory of fundamental bosons [37] or supersymmetric Chern-Simons theory. It also throws up a sharp question for the dual theory: what precise deformation of the parity-preserving Vasiliev theory is our system dual to? We hope to return to all these fascinating questions in the near future.

Note added: O. Aharony, G. Gur-Ari and R. Yacoby have recently demonstrated that massless scalar fundamental matter coupled to Chern-Simons theory also leads to a large $N$ fixed line of CFTs [37]. Using arguments very similar to those employed in part of section 3 of our paper, they have also argued that the higher spin currents in their theory are not renormalized as a function of the coupling constant. We thank O. Aharony for sharing his results with us prior to publication and also for several discussions on these issues over the last year.

\section{Free Energy on $\mathbb{R}^{2}$ at Finite Temperature}

In this section we will evaluate the free energy of our theory (a single species of massless fundamental fermions coupled to a Chern-Simons gauge field) in the t' Hooft large $N$ 
limit. The Euclidean action for our theory is

$$
S=\frac{i k}{4 \pi} \int \operatorname{Tr}\left(A d A+\frac{2}{3} A^{3}\right)+\int \bar{\psi} \gamma^{\mu} D_{\mu} \psi,
$$

Our theory is taken to be at temperature $T$, and lives on a spatial $\mathbb{R}^{2}$ whose regulated volume we denote as $V_{2}$.

In order to evaluate the free energy of our theory, we first fix a gauge. We work in the lightcone gauge $A_{-}=0$. This gauge is defined in terms of an analytic continuation from Lorentzian space. For this reason $A_{-}=0$ does not imply $A_{+}=0$. Alternatively one can work in Lorentzian space and analytically continue to Euclidean space (after integrating out the gauge fields) in (2.24). The gauge boson self interaction term vanishes in this gauge, a feature that enormously simplifies analysis. 8 Computations in pure Chern-Simons theory on $\mathbb{R}^{3}$ in this Euclidean continuation of the light-cone gauge were done previously in [42, [43].

In our analysis below we will encounter divergent integrals that need to be regulated. We choose to regulate all integrals in the scheme of dimensional reduction. More specifically we evaluate all integrals as follows. We evaluate $\gamma$ traces, $\epsilon$ contraction etc in $d=3$. This process leaves us with a set of scalar integrals. We then evaluate the resultant integrals by analytic continuation from $d=3-\epsilon$ dimensions. This regularization scheme is widely employed in the previous literature on Chern-Simons matter theories (see e.g. 20, 21, 6, 7]). It is manifestly Lorentz invariant, and also respects gauge invariance at least up to two-loops (see [21]). We will assume without proof in what follows that our regularization scheme is indeed gauge invariant. If this is indeed the case then the theory defined by this regularization scheme must be Lorentz invariant even though we work in a gauge that breaks Lorentz invariance. We will find some evidence for the Lorentz invariance of our final results giving some a posteriori evidence for our assumption that our regularization scheme respects gauge invariance.

As we will explain below, the finite temperature free energy of our theory is completely determined by the fermion self energy on $\mathbb{R}^{2} \times S^{1}$ (see $(2.52$ ) ). In order to evaluate the free energy we proceed as follows. As a preliminary step to our analysis, we first determine the exact fermion propagator of our theory on $\mathbb{R}^{3}$. We then determine the exact fermion propagator on $\mathbb{R}^{2} \times S^{1}$. Finally, we proceed to use this result to determine the free energy of our theory.

\footnotetext{
${ }^{8}$ This feature is also true of the more straightforward "temporal" or axial gauge $A_{3}=0$. Feynman diagrams in this gauge are, however, plagued by logarithmic divergences that we have found difficult to interpret and deal with. In contrast the divergences in the lightcone gauge employed in this subsection are relatively tame, and are easy to interpret and deal with. We thank S. Bhattacharyya and J. Bhattacharya for extensive discussions on perturbation theory and its divergences in Axial gauge.
} 


\subsection{Exact fermion propagator on $\mathbb{R}^{3}$}

In the planar limit, the exact fermion propagator in light-cone gauge receives contributions from a very simple class of diagrams. Indeed, the gluon vertex vanishes in our gauge, and moreover at large $N$ we can neglect fermion loops since the fermions are in the fundamental representation. Therefore, only rainbow diagrams contribute to the exact propagator. These can be resummed exactly by solving a Schwinger-Dyson equation which may be depicted diagrammatically as

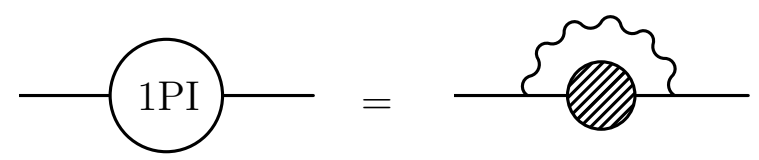

The planar fermion self energy.

This picture may be transcribed into equations as follows. Let the exact fermion propagator be given by

$$
\left\langle\psi(p)_{m} \bar{\psi}(-q)^{n}\right\rangle=\delta_{m}^{n} \frac{1}{i p_{\mu} \gamma_{\mu}+M_{\text {bare }}+\Sigma} \times(2 \pi)^{3} \delta(p-q) .
$$

Then

$$
\Sigma(p)=\frac{N}{2} \int \frac{d^{3} q}{(2 \pi)^{3}}\left(\gamma^{\mu} \frac{1}{i \gamma^{\alpha} q_{\alpha}+M_{\text {bare }}+\Sigma(q)} \gamma^{\nu}\right) G_{\mu \nu}(p-q)
$$

where

$$
\left\langle A_{\mu}^{a}(p) A_{\nu}^{b}(-q)\right\rangle=(2 \pi)^{3} \delta(p-q) G_{\mu \nu}(p) \delta^{a b}
$$

is the bare gluon propagator 9 Here $M_{\text {bare }}$ is the mass term that appears in the bare Lagrangian. In what follows we will adjust $M_{\text {bare }}$ to ensure that the physical fermion mass vanishes (this choice corresponds to tuning the theory to the conformality). Note that with our definition of $\Sigma$ in $(2.2)$, the sum of 1PI diagrams is given by $-\Sigma$.

The equation (2.3) applies in fact in any ghost free gauge in which all gluon interactions vanish. In this section we will solve $(2.3)$ in the lightcone gauge.

\subsubsection{Exact solution of the gap equation}

In the this subsection we will solve the gap equation 2.3 in light-cone gauge (see Appendix A for a detailed listing of our conventions, propagators etc in lightcone

\footnotetext{
${ }^{9}$ The factor of $\frac{1}{2}$ in 2.3 has its origin in the $\frac{1}{2}$ on the RHS of A.3.
} 
gauge):

$\Sigma(p)=-i 2 \pi \lambda \int \frac{d^{3} q}{(2 \pi)^{3}}\left(\gamma^{3} \frac{1}{i \gamma^{\mu} q_{\mu}+M_{\text {bare }}+\Sigma(q)} \gamma^{+}-\gamma^{+} \frac{1}{i \gamma^{\mu} q_{\mu}+M_{\text {bare }}+\Sigma(q)} \gamma^{3}\right) \frac{1}{(p-q)^{+}}$.

Let us first better understand the matrix structure of the self energy $\Sigma$. Let $A$ represent an arbitrary $2 \times 2$ matrix

$$
A=A_{I} I+A_{+} \gamma^{+}+A_{-} \gamma^{-}+A_{3} \gamma^{3}
$$

For use below and in later sections we define the matrix valued functions of $A, H_{+}(A)$ and $H_{-}(A)$ as

$$
\begin{gathered}
H_{+}(A) \equiv \gamma^{3} A \gamma^{+}-\gamma^{+} A \gamma^{3}=2\left(A_{I} \gamma^{+}-A_{-} I\right) \\
H_{-}(A) \equiv \gamma^{3} A \gamma^{-}-\gamma^{-} A \gamma^{3}=2\left(-A_{I} \gamma^{-}+A_{+} I\right)
\end{gathered}
$$

The gap equation (2.4) may be rewritten as

$$
\Sigma(p)=-i 2 \pi \lambda \int \frac{d^{3} q}{(2 \pi)^{3}}\left(H_{+}\left[\frac{1}{i \gamma^{\mu} q_{\mu}+M_{\text {bare }}+\Sigma(q)}\right] \frac{1}{(p-q)^{+}}\right) .
$$

In the discussion which follows we will sometime abbreviate the notation $\Sigma(p)$ to $\Sigma$ for brevity. Now let

$$
\Sigma=i \Sigma_{\mu} \gamma^{\mu}+\Sigma_{I} I-M_{\text {bare }} I
$$

Using

$$
\frac{1}{i \gamma^{\mu}\left(q_{\mu}+\Sigma_{\mu}\right)+\Sigma_{I}}=\frac{-i \gamma^{\mu}\left(p_{\mu}+\Sigma_{\mu}\right)+\Sigma_{I}}{(p+\Sigma)^{2}+\Sigma_{I}^{2}}
$$

together with (2.5), we may rewrite (2.7) as

$$
\Sigma(p)=-i 4 \pi \lambda \int \frac{d^{3} q}{(2 \pi)^{3}} \frac{\gamma^{+} \Sigma_{I}+i I(q+\Sigma(q))_{-}}{\left(q_{\mu}+\Sigma_{\mu}(q)\right)\left(q^{\mu}+\Sigma^{\mu}(q)\right)+\Sigma_{I}(q)^{2}} \frac{1}{(p-q)^{+}}
$$

Plugging (2.8) into the LHS of (2.9) and equating the coefficients of linearly independent matrices, it follows immediately that

$$
\Sigma_{-}=\Sigma_{3}=0 .
$$

And that

$$
\begin{aligned}
\Sigma_{+}(p) & =-4 \pi \lambda \int \frac{d^{3} q}{(2 \pi)^{3}} \frac{\Sigma_{I}}{\left((q+\Sigma(q))^{2}+\Sigma_{I}(q)^{2}\right)} \frac{1}{(p-q)^{+}} \\
\Sigma_{I}(p)-M_{\text {bare }} & =4 \pi \lambda \int \frac{d^{3} q}{(2 \pi)^{3}} \frac{q_{-}}{\left((q+\Sigma(q))^{2}+\Sigma_{I}(q)^{2}\right)} \frac{1}{(p-q)^{+}}
\end{aligned}
$$


What can we say about the dependence of $\Sigma_{+}(p)$ and $\Sigma_{I}(p)$ on $p$ ? First note that the that the RHS of the two equations in (2.11) is independent of $p^{3}$. It follows that $\Sigma$ is a function only of the in plane momenta $p^{1}$ and $p^{2}$ but is independent of $p^{3}$. Rotational invariance in the 12 plane and requirement of conformality (i.e. the requirement that no mass scale enter the physical propagator) then together completely fix the momentum dependence of $\Sigma_{+}$and $\Sigma_{I}$ :

$$
\begin{aligned}
\Sigma_{I}(p) & =f_{0} p_{s} \\
\Sigma_{+}(p) & =p_{+} g_{0}=p^{-} g_{0}
\end{aligned}
$$

where

$$
p_{s}=\sqrt{p_{1}^{2}+p_{2}^{2}}=\sqrt{2}\left|p^{-}\right|=\sqrt{2}\left|p^{+}\right|
$$

and $f_{0}$ and $g_{0}$ are dimensionless numbers (that are functions of the coupling constant $\lambda)$.

Plugging (2.13) and 2.10 into 2.11) we find

$$
\begin{aligned}
g_{0} & =-\frac{4 \pi \lambda}{p^{-}} \int \frac{d^{3} q}{(2 \pi)^{3}} \frac{q_{s} f_{0}}{q_{3}^{2}+q_{s}^{2}\left(1+g_{0}+f_{0}^{2}\right)} \frac{1}{(p-q)^{+}} \\
f_{0}|p|-M_{\text {bare }} & =4 \pi \lambda \int \frac{d^{3} q}{(2 \pi)^{3}} \frac{q^{+}}{q_{3}^{2}+q_{s}^{2}\left(1+g_{0}+f_{0}^{2}\right)} \frac{1}{(p-q)^{+}}
\end{aligned}
$$

We will now proceed to determine the numbers $g_{0}$ and $f_{0}$ as functions of $\lambda$.

We first note that the integrals on the RHS of (2.14) are (power counting) linearly divergent. In order to proceed we need to regulate these divergences. We adopt a regulator that is manifestly Lorentz invariant as well as plausibly gauge invariant. Our regularization procedure is simply the following: we analytically continue all diagrams to $3-\epsilon$ dimensions. Two of these dimensions span the 1-2 plane. We integrate over the remaining $1-\epsilon$ dimensions using the formula

$$
\int_{-\infty}^{\infty} \frac{d^{1-\epsilon} x}{a^{2}+x^{2}}=\frac{A^{\epsilon} \pi}{|a|^{1+\epsilon}}
$$

Here $A$ is a number of order unity which is easily computed. However, as we will see below, none of the integrals we compute in this paper have a $\frac{1}{\epsilon}$ type divergence (this corresponds to an absence of logarithmic divergences, were we to use a momentum cutoff). The only effect of the dimensional regularization cut off procedure, employed in our paper, is to discard linear (and below also cubic) divergences in a gauge and Lorentz invariant manner. For that reason, when we take $\epsilon \rightarrow 0$ at the end of the calculation, we effectively set $A$ to unity. Hence we immediately set $A$ to unity instead of carrying it around in our computation. The regularization procedure we employ here 
is essentially the dimensional reduction scheme used in [21], adapted to our light-cone gauge.

Using (2.15) in 2.14 yields

$$
\begin{aligned}
g_{0} & =-\frac{2 \pi \lambda}{p^{-}} \frac{f_{0}}{\sqrt{1+g_{0}+f_{0}^{2}}} \int \frac{d^{2} q}{(2 \pi)^{2}} \frac{1}{q_{s}^{\epsilon}(p-q)^{+}} \\
f_{0} p_{s}-M_{\text {bare }} & =2 \pi \lambda \frac{1}{\sqrt{1+g_{0}+f_{0}^{2}}} \int \frac{d^{2} q}{(2 \pi)^{2}} \frac{q^{+}}{q_{s}^{1+\epsilon}(p-q)^{+}}
\end{aligned}
$$

In order to do the integrals we move to polar coordinates in the 12 plane. The integrals we need to evaluate are

$$
\begin{aligned}
& \int \frac{d^{2} q}{(2 \pi)^{2}} \frac{q^{+}}{q_{s}^{1+\epsilon}(p-q)^{+}}=\frac{1}{(2 \pi)^{2}} \int_{0}^{\infty} q_{s}^{1-\epsilon} d q \int_{0}^{2 \pi} d \theta \frac{p_{s} \cos \theta-q_{s}}{q_{s}^{2}+p_{s}^{2}-2 p_{s} q_{s} \cos \theta} \\
& \int \frac{d^{2} q}{(2 \pi)^{2} p^{-}} \frac{q_{s}^{1-\epsilon}}{q_{s}^{\epsilon}(p-q)^{+}}=\frac{2}{(2 \pi)^{2} p_{s}} \int_{0}^{\infty} q_{s}^{1-\epsilon} d q \int_{0}^{2 \pi} d \theta \frac{p_{s}-q_{s} \cos \theta}{q_{s}^{2}+p_{s}^{2}-2 p_{s} q_{s} \cos \theta}
\end{aligned}
$$

Using contour techniques it is not difficult to verify that for $q>p$

$$
\begin{aligned}
& \int_{0}^{2 \pi} \frac{d \theta}{q^{2}+p^{2}-2 p q \cos \theta}=\frac{2 \pi}{q^{2}-p^{2}} \\
& \int_{0}^{2 \pi} \frac{d \theta \cos \theta}{q^{2}+p^{2}-2 p q \cos \theta}=\frac{p}{q} \frac{2 \pi}{q^{2}-p^{2}}
\end{aligned}
$$

It follows that

$$
\begin{aligned}
& \int_{0}^{2 \pi} d \theta \frac{p_{s} q_{s} \cos \theta-q_{s}^{2}}{q_{s}^{2}+p_{s}^{2}-2 p_{s} q_{s} \cos \theta}=0 \quad\left(q_{s}<p_{s}\right) \\
& \int_{0}^{2 \pi} d \theta \frac{p_{s} q_{s} \cos \theta-q_{s}^{2}}{q_{s}^{2}+p_{s}^{2}-2 p_{s} q_{s} \cos \theta}=-2 \pi \quad\left(q_{s}>p_{s}\right) \\
& \int_{0}^{2 \pi} d \theta \frac{p_{s} q_{s}-q_{s}^{2} \cos \theta}{q_{s}^{2}+p_{s}^{2}-2 p_{s} q_{s} \cos \theta}=2 \pi \quad\left(q_{s}<p_{s}\right) \\
& \int_{0}^{2 \pi} d \theta \frac{p_{s} q_{s}-q_{s}^{2} \cos \theta}{q_{s}^{2}+p_{s}^{2}-2 p_{s} q_{s} \cos \theta}=0 \quad\left(q_{s}>p_{s}\right) .
\end{aligned}
$$

It follows that 2.16 reduces to

$$
\begin{aligned}
g_{0} & =-\frac{\lambda f_{0}}{\sqrt{1+g_{0}+f_{0}^{2}}} \\
f_{0} p_{s}-M_{\text {bare }} & =-\frac{\lambda}{\sqrt{1+g_{0}+f_{0}^{2}}} \int_{p_{s}}^{\infty} q^{-\epsilon} d q=\frac{\lambda}{\sqrt{1+g_{0}+f_{0}^{2}}} p_{s}
\end{aligned}
$$

It follows from 2.20 that $M_{\text {bare }}=0$ (this is a consequence of our use of dimensional regularization; $M_{\text {bare }}$ is linearly divergent in a cut off regulator, as is clear from the 
second of $(2.20)$ ). The remaining equations reduce to

$$
\begin{aligned}
& g_{0}=-\frac{\lambda f_{0}}{\sqrt{1+g_{0}+f_{0}^{2}}} \\
& f_{0}=\frac{\lambda}{\sqrt{1+g_{0}+f_{0}^{2}}}
\end{aligned}
$$

The solution to 2.21 is remarkably simple

$$
\begin{aligned}
f_{0} & =\lambda \\
g_{0} & =-\lambda^{2} \\
g_{0}+f_{0}^{2} & =0
\end{aligned}
$$

In other words, the self energy receives contributions from one and two loop graphs but not at any higher order in perturbation theory! This completes our solution of the gap equation.

We emphasize that our final result 2.22 depends in a crucial way on our choice of regularization scheme. Were we, for instance to regulate the integrals (2.14) by

modifying the gauge boson propagator with a Gaussian damping factor $e^{-\frac{(p-q)^{2}}{2 \Lambda^{2}}}$, then we would have found $g_{0}=0$ (from the integral over the angle in the vector $p-q$ ). It is of course quite clear that a crude cut off on the gauge boson momentum does not preserve either gauge or Lorentz invariance. We hope on the other hand that the more sophisticated dimensional regularization scheme preserves both these symmetries. This assumption, which is as yet unproved, is the main weakness in the analysis presented in this section. We will return to this point at the end of the section.

In summary, the exact fermion propagator, at leading order in large $N$, is given by

$$
\left\langle\psi(p)_{m} \bar{\psi}(-q)^{n}\right\rangle=\delta_{m}^{n} \frac{1}{i p_{3} \gamma^{3}+i p_{-} \gamma^{-}+i\left(1-\lambda^{2}\right) p_{+} \gamma^{+}+\lambda p_{s}} \times(2 \pi)^{3} \delta(p-q)
$$

(Here $m, n$ are colour indices and we have suppressed the spinor indices).

\subsubsection{Rederivation of the gap equation as a Schwinger-Dyson equation}

The starting point of our analysis in this subsection is the path integral representation of the partition function

$$
Z=\int D \psi D \bar{\psi} D A_{\mu} e^{-S}
$$

where the action $S$ is the Euclidean space lightcone gauge action for our field theory, listed explicitly in A.6. The gauge fields appear quadratically in in A.6 and may 
be integrated out. Integrating out the gauge field from A.6 yields the path integra ${ }^{10}$

$$
Z=\int D \psi D \bar{\psi} e^{-S}
$$

where the action $S$ is now given by

$$
\begin{aligned}
S & =i \int \frac{d^{3} p}{(2 \pi)^{3}} \bar{\psi}(-p) \gamma^{\mu} p_{\mu} \psi(p) \\
& +\frac{2 \pi i}{k} \int \frac{d^{3} p}{(2 \pi)^{3}} \frac{d^{3} r}{(2 \pi)^{3}} \frac{d^{3} q}{(2 \pi)^{3}} \frac{1}{q^{+}} \bar{\psi}^{m}(-p) \gamma^{+} \psi_{n}(p-q) \bar{\psi}^{n}(-r) \gamma^{3} \psi_{m}(r+q)
\end{aligned}
$$

Let us pause to note that, starting from the bilocal action (2.24), one can conveniently derive the Schwinger-Dyson equation for the fermion self-energy (as in [46]) via

$$
\begin{aligned}
0 & =\int D \psi D \bar{\psi} \frac{\delta}{\delta \bar{\psi}^{m}(-p)}\left(e^{-S} \bar{\psi}^{n}\left(p^{\prime}\right)\right) \\
& =\int D \psi D \bar{\psi}\left(\delta_{m}^{n} \delta^{3}\left(p^{\prime}+p\right)-\frac{\delta S}{\delta \bar{\psi}^{m}(-p)} \bar{\psi}^{n}\left(p^{\prime}\right)\right) e^{-S}
\end{aligned}
$$

which gives the following relation involving the exact fermion propagator and four-point functions,

$$
\begin{aligned}
(2 \pi)^{3} \delta^{3}\left(p^{\prime}+p\right)= & i p_{\mu} \gamma^{\mu}\left\langle\psi_{m}(p) \bar{\psi}^{n}\left(p^{\prime}\right)\right\rangle \\
& +\frac{2 \pi i}{k} \int \frac{d^{3} r}{(2 \pi)^{3}} \frac{d^{3} q}{(2 \pi)^{3}} \frac{1}{q^{+}} \gamma^{+}\left\langle\psi_{a}(p-q) \bar{\psi}^{a}(-r) \gamma^{3} \psi_{m}(r+q) \bar{\psi}^{n}\left(p^{\prime}\right)\right\rangle \\
& -\frac{2 \pi i}{k} \int \frac{d^{3} r}{(2 \pi)^{3}} \frac{d^{3} q}{(2 \pi)^{3}} \frac{1}{q^{+}} \gamma^{3}\left\langle\psi_{a}(p-q) \bar{\psi}^{a}(-r) \gamma^{+} \psi_{m}(r+q) \bar{\psi}^{n}\left(p^{\prime}\right)\right\rangle .
\end{aligned}
$$

In the large- $N$ limit, this factorizes to yield

$$
\begin{aligned}
\left\langle\psi_{m}(p) \bar{\psi}^{n}\left(p^{\prime}\right)\right\rangle= & \frac{1}{i p_{\mu} \gamma^{\mu}}(2 \pi)^{3} \delta^{3}\left(p^{\prime}+p\right) \\
& -\frac{1}{i p_{\mu} \gamma^{\mu}} \frac{2 \pi i}{k} \int \frac{d^{3} r}{(2 \pi)^{3}} \frac{d^{3} q}{(2 \pi)^{3}} \frac{1}{q^{+}} \gamma^{+}\left\langle\psi_{a}(p-q) \bar{\psi}^{a}(-r)\right\rangle\left\langle\gamma^{3} \psi_{m}(r+q) \bar{\psi}^{n}\left(p^{\prime}\right)\right\rangle \\
& +\frac{1}{i p_{\mu} \gamma^{\mu}} \frac{2 \pi i}{k} \int \frac{d^{3} r}{(2 \pi)^{3}} \frac{d^{3} q}{(2 \pi)^{3}} \frac{1}{q^{+}} \gamma^{3}\left\langle\psi_{a}(p-q) \bar{\psi}^{a}(-r)\right\rangle \gamma^{+}\left\langle\psi_{m}(r+q) \bar{\psi}^{n}\left(p^{\prime}\right)\right\rangle
\end{aligned}
$$

which, upon substituting 2.2 , gives the gap equation:

$$
\Sigma(p)=-2 \pi i \lambda \int \frac{d^{3} q}{(2 \pi)^{3}} \frac{1}{p^{+}-q^{+}} H_{+}\left(\frac{1}{i\left(q_{\mu}\right) \gamma^{\mu}+\Sigma(q)}\right) .
$$

\footnotetext{
${ }^{10}$ In integrating out the gauge field, we absorb the factor coming from the determinant of the gauge field kinetic operator in the normalization of the path integral. In other words, we normalize the pure Chern-Simons partition function to 1.
} 


\subsubsection{Rewriting the field theory as a path integral over singlet fields}

In this subsection we will reformulate the path integral that evaluates the partition function of our field theory as a path integral over singlest fields. The new path integral is weakly coupled in the large $N$ limit (the action in terms of the new variables is proportional to $N$ ). The gap equation (2.4) follows as the classical equation of motion of this large $N$ action.

While the work out of this subsection is considerably more complicated than that of subsection (2.1.1) it has one significant advantage; it reveals how the solution of the gap equation is related to the value the partition function of the theory. While the value of the partition function is of no physical significance for the theory on $\mathbb{R}^{3}$, it is of great significance on $\mathbb{R}^{2} \times S^{1}$ (as it determines the thermal partition function of the theory on $\mathbb{R}^{2}$ ). For this reason the results of this subsection will prove very useful in our discussion of the finite temperature partition function in the next section.

We now introduce some convenient shorthand notation. Let

$$
M(P, q)=\frac{1}{N} \int \frac{d q^{3}}{2 \pi} \psi_{m}\left(\frac{P}{2}+q\right) \bar{\psi}^{m}\left(\frac{P}{2}-q\right) .
$$

$M$ is a $2 \times 2$ matrix in spinor space but a singlet in colour space. While one of its arguments, $P$, is a 3 momentum, its second argument $q$ is a 2 momentum (in integral on the RHS of $(2.30)$ is over the 3 component of $q)$. (2.24) may be rewritten as

$$
\begin{aligned}
S & =i \int \frac{d^{3} p}{(2 \pi)^{3}} \bar{\psi}(-p) \gamma^{\mu} p_{\mu} \psi(p) \\
& -\frac{2 \pi i N^{2}}{k} \int \frac{d^{3} P}{(2 \pi)^{3}} \frac{d^{2} q}{(2 \pi)^{2}} \frac{d^{2} q^{\prime}}{(2 \pi)^{2}} \frac{1}{\left(q-q^{\prime}\right)^{+}} \operatorname{Tr}\left(M(P, q) \gamma^{+} M\left(-P, q^{\prime}\right) \gamma^{3}\right) \\
& =i \int \frac{d^{3} p}{(2 \pi)^{3}} \bar{\psi}(-p) \gamma^{\mu} p_{\mu} \psi(p) \\
& -\frac{\pi i N^{2}}{k} \int \frac{d^{3} P}{(2 \pi)^{3}} \frac{d^{2} q}{(2 \pi)^{2}} \frac{d^{2} q^{\prime}}{(2 \pi)^{2}} \frac{1}{\left(q-q^{\prime}\right)^{+}} \operatorname{Tr}\left[\left(M(P, q) \gamma^{+} M\left(-P, q^{\prime}\right) \gamma^{3}\right)-\left(M(P, q) \gamma^{3} M\left(-P, q^{\prime}\right) \gamma^{+}\right)\right]
\end{aligned}
$$

(the flip in sign is due to the fact that we had to take one fermionic field through three others). Expanding the matrix $M$ in a complete basis of $2 \times 2$ matrices

$$
M=M_{+} \gamma^{+}+M_{-} \gamma^{-}+M_{3} \gamma^{3}+M_{I} I
$$

we find that 2.31 reduces to

$$
\begin{aligned}
S & =i \int \frac{d^{3} p}{(2 \pi)^{3}} \bar{\psi}(-p) \gamma^{\mu} p_{\mu} \psi(p) \\
& +\frac{8 \pi i N^{2}}{k} \int \frac{d^{3} P}{(2 \pi)^{3}} \frac{d^{2} q}{(2 \pi)^{2}} \frac{d^{2} q^{\prime}}{(2 \pi)^{2}} \frac{1}{\left(q-q^{\prime}\right)^{+}} M_{-}(P, q) M_{I}\left(-P, q^{\prime}\right)
\end{aligned}
$$


where we have used

$$
\operatorname{Tr}\left(\left[\gamma^{-}, \gamma^{+}\right] \gamma^{3}\right)=-4
$$

Note in particular that $M_{+}$and $M_{3}$ drop out of this expression.

We will now rewrite the interaction term (the term quadratic in $M$ ) in (2.33) in terms of a Lagrange multiplier field

$$
\Sigma=\Sigma_{+} \gamma^{+}+\Sigma_{I} I
$$

where $\Sigma$ will turn out to be the self energy of the fermion field. To this end we define the "inverse" Greens function $G^{-1}(p)$ by the requirement that

$$
\int \frac{d^{2} q}{(2 \pi)^{2}} G^{-1}(p-q) \frac{1}{(q-r)^{+}}=(2 \pi)^{2} \delta^{2}(p-r)
$$

Note that $G^{-1}$ is an odd function of its argument. Note also that

$$
\int \frac{d^{2} r}{(2 \pi)^{2}} \frac{1}{(q-r)^{+}} G^{-1}(r-p)=(2 \pi)^{2} \delta^{2}(q-p)
$$

These are the only properties of $G^{-1}$ that we will need in this paper; in particular we will never need the explicit form of the function $G^{-1}$.

Now it is obvious that

$$
Z=\frac{\int D \psi D \Sigma_{-} D \Sigma_{I} e^{-(S+E)}}{\int D \Sigma_{-} D \Sigma_{I} e^{-E}}
$$

where we have chosen

$$
\begin{aligned}
E & =2 \times \frac{N}{4 \pi i \lambda} \int \frac{d^{3} P}{(2 \pi)^{3}} \frac{d^{2} q}{(2 \pi)^{2}} \frac{d^{2} q^{\prime}}{(2 \pi)^{2}} \\
& {\left[\left(\Sigma_{+}(P, q)-4 \pi i \lambda \int \frac{d^{2} r}{(2 \pi)^{2}} M_{I}(P, r) \frac{1}{(r-q)^{+}}\right) \times G^{-1}\left(q-q^{\prime}\right)\right.} \\
& \left.\times\left(\Sigma_{I}\left(-P, q^{\prime}\right)-4 \pi i \lambda \int \frac{d^{2} r^{\prime}}{(2 \pi)^{2}} \frac{1}{\left(q^{\prime}-r^{\prime}\right)^{+}} M_{-}\left(-P, r^{\prime}\right)\right)\right]
\end{aligned}
$$

Note that $E$ is a function of the two new Lagrange multiplier fields $\Sigma_{-}$and $\Sigma_{I}$. The path integral in the denominator in 2.38 is simply a number of order unity and we will omit to write it in the equations that follow. The effective action in the numerator, $S+E$, evaluates to

$$
\begin{aligned}
S & =i \int \frac{d^{3} p}{(2 \pi)^{3}} \bar{\psi}(-p) \gamma^{\mu} p_{\mu} \psi(p) \\
& +\int \frac{d^{3} P}{(2 \pi)^{3}} \frac{d^{3} q}{(2 \pi)^{3}} \bar{\psi}\left(\frac{P}{2}-q\right) \Sigma(-P, q) \psi\left(\frac{P}{2}+q\right) \\
& +\frac{N}{2 \pi i \lambda} \int \frac{d^{3} P}{(2 \pi)^{3}} \frac{d^{2} q}{(2 \pi)^{2}} \frac{d^{2} q^{\prime}}{(2 \pi)^{2}} \Sigma_{+}(P, q) G^{-1}\left(q-q^{\prime}\right) \Sigma_{I}\left(-P, q^{\prime}\right)
\end{aligned}
$$


In the second line above we have used the fact that

$$
-2\left(\Sigma_{+} M_{-}+\Sigma_{I} M_{I}\right)=-\operatorname{Tr} \Sigma M=-\frac{1}{N} \int \frac{d q_{3}}{2 \pi} \operatorname{Tr} \Sigma \psi \bar{\psi}=\frac{1}{N} \int \frac{d q_{3}}{2 \pi} \bar{\psi} \Sigma \psi
$$

Using 2.34 the last line in 2.33 may be rewritten as a trace, yielding

$$
\begin{aligned}
S & =i \int \frac{d^{3} p}{(2 \pi)^{3}} \bar{\psi}(-p) \gamma^{\mu} p_{\mu} \psi(p) \\
& +\int \frac{d^{3} P}{(2 \pi)^{3}} \frac{d^{3} q}{(2 \pi)^{3}} \bar{\psi}\left(\frac{P}{2}-q\right) \Sigma(-P, q) \psi\left(\frac{P}{2}+q\right) \\
& -\frac{N}{8 \pi i \lambda} \int \frac{d^{3} P}{(2 \pi)^{3}} \frac{d^{2} q}{(2 \pi)^{2}} \frac{d^{2} q^{\prime}}{(2 \pi)^{2}} G^{-1}\left(q-q^{\prime}\right) \operatorname{Tr}\left(\gamma^{-} \Sigma(P, q) \gamma^{3} \Sigma\left(-P, q^{\prime}\right)\right)
\end{aligned}
$$

The action (2.41) may be rewritten as

$$
\begin{aligned}
S & =\int \frac{d^{3} P}{(2 \pi)^{3}} \frac{d^{3} q}{(2 \pi)^{3}} \bar{\psi}\left(\frac{P}{2}-q\right)\left((2 \pi)^{3} \delta^{3}(P) i \gamma^{\mu} q_{\mu}+\Sigma(-P, q)\right) \psi\left(\frac{P}{2}+q\right) \\
& -\frac{N}{8 \pi i \lambda} \int \frac{d^{3} P}{(2 \pi)^{3}} \frac{d^{2} q}{(2 \pi)^{2}} \frac{d^{2} q^{\prime}}{(2 \pi)^{2}} G^{-1}\left(q-q^{\prime}\right) \operatorname{Tr}\left(\gamma^{-} \Sigma(P, q) \gamma^{3} \Sigma\left(-P, q^{\prime}\right)\right)
\end{aligned}
$$

The dependence of 2.42 on fermionic fields is quadratic, so the later may be integrated out. Performing this operation yields

$$
Z=\int D \Sigma_{-} D \Sigma_{I} e^{-S}
$$

where

$$
\begin{aligned}
S & =-N \operatorname{Tr} \ln \left((2 \pi)^{3} \delta^{3}(P) i \gamma^{\mu} q_{\mu}+\Sigma(-P, q)\right) \\
& -\frac{N}{8 \pi i \lambda} \int \frac{d^{3} P}{(2 \pi)^{3}} \frac{d^{2} q}{(2 \pi)^{2}} \frac{d^{2} q^{\prime}}{(2 \pi)^{2}} G^{-1}\left(q-q^{\prime}\right) \operatorname{Tr}\left(\gamma^{-} \Sigma(P, q) \gamma^{3} \Sigma\left(-P, q^{\prime}\right)\right)
\end{aligned}
$$

Notice that 2.44) is written purely in terms of singlet fields, and is multiplied by an overall factor of $N$. 2.44) represents an exact rewriting of the partition function of the original theory as a partition function over the singlet fields $\Sigma$; this path integral is weakly coupled in the large $N$ limit.

The action, 2.44, is somewhat formal, as it is written in terms of a determinant over an infinite dimensional matrix. However the equivalent of (2.44) is much simpler for translationally invariant $\Sigma$ configurations of the form

$$
\Sigma(P, q)=(2 \pi)^{3} \delta(P) \Sigma(q)
$$


The form of this action is perhaps most clearly obtained by retreating to 2.42 , which reduces, for translationally $\Sigma$ configurations to

$$
S=\int \frac{d^{3} p}{(2 \pi)^{3}} \bar{\psi}(-p)\left[i \gamma^{\mu} p_{\mu}+\Sigma(p)\right] \psi(p)-\frac{N V}{8 \pi i \lambda} \int \frac{d^{2} q}{(2 \pi)^{2}} \frac{d^{2} q^{\prime}}{(2 \pi)^{2}} G^{-1}\left(q-q^{\prime}\right) \operatorname{Tr}\left(\gamma^{-} \Sigma(q) \gamma^{3} \Sigma\left(q^{\prime}\right)\right)
$$

$V$ here is a factor of the volume of spacetime, and we have used

$$
\left[(2 \pi)^{3} \delta(P)\right]^{2}=V(2 \pi)^{3} \delta(P)
$$

in the last term of the last line.

Integrating out the fermions in 2.45) yields a very explicit special case of 2.44

$S=-N V \int \frac{d^{3} q}{(2 \pi)^{3}} \operatorname{Tr} \ln \left(i \gamma^{\mu} q_{\mu}+\Sigma(p)\right)-\frac{N V}{8 \pi i \lambda} \int \frac{d^{2} q}{(2 \pi)^{2}} \frac{d^{2} q^{\prime}}{(2 \pi)^{2}} G^{-1}\left(q-q^{\prime}\right) \operatorname{Tr}\left(\gamma^{-} \Sigma(q) \gamma^{3} \Sigma\left(q^{\prime}\right)\right)$

While all terms in the action in $(2.46)$ are proportional to $N$, the fields in that action are gauge singlets. At leading order in the large $N$ expansion it follows that the free energy for our theory may evaluated simply by minimizing (2.46) w.r.t. $\Sigma$. The variational equation we encounter in this minimization process is

$$
\int \operatorname{Tr}\left[\frac{d^{2} q}{(2 \pi)^{2}} \delta \Sigma[q] \int\left(\frac{d^{2} q^{\prime}}{(2 \pi)^{2}} \frac{-1}{8 \pi i \lambda} G^{-1}\left(q-q^{\prime}\right)\left(\gamma^{3} \Sigma\left(q^{\prime}\right) \gamma^{-}-\gamma^{-} \Sigma\left(q^{\prime}\right) \gamma^{3}\right)-\int \frac{d q^{3}}{2 \pi} \frac{1}{i \gamma^{\mu} q_{\mu}+\Sigma}\right)\right]=0
$$

In terms of the function $H_{-}$defined 2.6

$$
\int \operatorname{Tr}\left[\frac{d^{2} q}{(2 \pi)^{2}} \delta \Sigma[q] \int\left(\frac{d^{2} q^{\prime}}{(2 \pi)^{2}} \frac{-1}{8 \pi i \lambda} G^{-1}\left(q-q^{\prime}\right)\left(H_{-}\left(\Sigma\left(q^{\prime}\right)\right)\right)-\int \frac{d q^{3}}{2 \pi} \frac{1}{i \gamma^{\mu} q_{\mu}+\Sigma}\right)\right]=0
$$

The equation 2.48) is of the form

$$
\int \operatorname{Tr}\left[\frac{d^{2} q}{(2 \pi)^{2}} \delta \Sigma[q] B(q)\right]=0
$$

where

$$
B(q)=\int\left(\frac{d^{2} q^{\prime}}{(2 \pi)^{2}} \frac{-1}{8 \pi i \lambda} G^{-1}\left(q-q^{\prime}\right)\left(H_{-}\left(\Sigma\left(q^{\prime}\right)\right)\right)-\int \frac{d q^{3}}{2 \pi} \frac{1}{i \gamma^{\mu} q_{\mu}+\Sigma}\right)
$$

As $\delta \Sigma$ is an arbitrary matrix of the form 2.35 it follows that

$$
B_{-}(q)=B_{I}(q)=0
$$

i.e. that

$$
H_{+}(B)=0
$$


(see (2.5)) Using the fact that

$$
H_{+}\left(H_{-}(\Sigma)\right)=4 \Sigma
$$

and integrating both sides of 2.48 against the kernel $\frac{1}{(p-q)^{+}}$and using the defining property of the function $G^{-1}$, it follows from $\left.H_{+}(B)\right)=0$ that

$$
\Sigma(p)=-2 \pi i \lambda \int \frac{d^{3} q}{(2 \pi)^{3}}\left(\gamma^{3} \frac{1}{i \gamma^{\mu} q_{\mu}+\Sigma} \gamma^{+}-\gamma^{+} \frac{1}{i \gamma^{\mu} q_{\mu}+\Sigma} \gamma^{3}\right) \frac{1}{(p-q)^{+}}
$$

in precise agreement with 2.4 .

The value of the Euclidean action on the saddle point, 2.46), may be rewritten as

$$
\begin{aligned}
S & =-N V \int \frac{d^{3} q}{(2 \pi)^{3}} \operatorname{Tr} \ln \left(i \gamma^{\mu} q_{\mu}+\Sigma(q)\right)+\frac{N V}{16 \pi i \lambda} \int \frac{d^{2} q}{(2 \pi)^{2}} \frac{d^{2} q^{\prime}}{(2 \pi)^{2}} G^{-1}\left(q-q^{\prime}\right) \operatorname{Tr}\left(H_{-}[\Sigma(q)] \Sigma\left(q^{\prime}\right)\right) \\
& =-N V \int \frac{d^{3} q}{(2 \pi)^{3}} \operatorname{Tr}\left[\ln \left(i \gamma^{\mu} q_{\mu}+\Sigma(q)\right)+\frac{1}{8} H_{-}(\Sigma(q)) H_{+}\left(\left(\frac{1}{i \gamma^{\mu} q_{\mu}+\Sigma(q)}\right)\right)\right] \\
& =-N V \int \frac{d^{3} q}{(2 \pi)^{3}} \operatorname{Tr}\left[\ln \left[i \gamma^{\mu} q_{\mu}+\Sigma(q)\right]-\frac{1}{2} \Sigma(q)\left(\frac{1}{i \gamma^{\mu} q_{\mu}+\Sigma(q)}\right)\right]
\end{aligned}
$$

where we have used the equation of motion in going from first to the second line. In going from the second to the third line we have used the fact that for an arbitrary matrix $A$

$$
\operatorname{Tr}\left(H_{-}(\Sigma) H_{+}(A)\right)=-4 \operatorname{Tr}(\Sigma A) .
$$

\subsubsection{Diagrammatic expansion for the vacuum energy}

The result (2.51), expressing the vacuum energy in terms of the exact fermion selfenergy $\Sigma(p)$, has a clear diagrammatic interpretation. The perturbative planar diagrammatic expansion takes the form

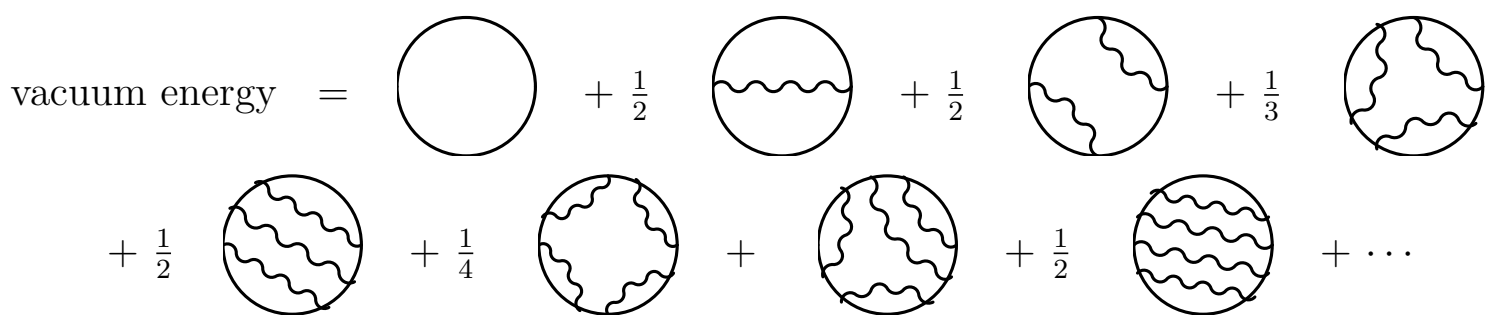

One may verify that, in terms of exact planar fermion self energy, the above expansion can be written as 


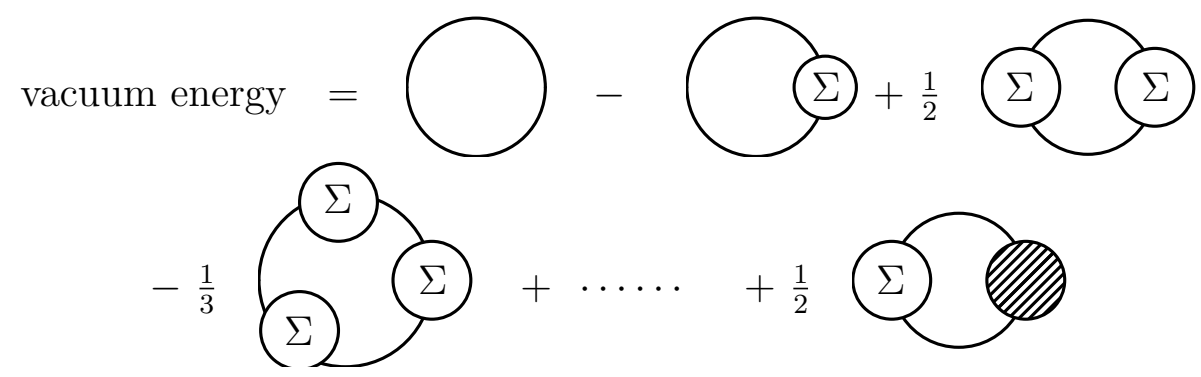

where the shaded blob represents the exact planar fermion propagator $(i \not q+\Sigma)^{-1}$. In particular, the second term in the last line of 2.51$),-\frac{1}{2} \operatorname{Tr}\left[\Sigma(i \not q+\Sigma)^{-1}\right]$ is precisely such that the correct symmetry factors are restored.

\subsection{The finite temperature theory}

In this section we study the logarithm of the path integral of our system on $R^{2} \times S^{1}$ where the circumference of the $S^{1}$ is taken to be $\beta$. This path integral determines the free energy of the field theory at temperature $T=\beta^{-1}$.

The formulas that determine the path integral on $\mathbb{R}^{2} \times S^{1}$ are straightforward generalizations of the formulas on $\mathbb{R}^{3}$. Every equation in subsection 2.1 .3 carries through with the replacement

$$
\begin{aligned}
\int \frac{d p_{3}}{(2 \pi)} f\left(p_{3}\right) & \rightarrow \frac{1}{\beta} \sum_{n \in Z+\frac{1}{2}} f\left(\frac{2 \pi n}{\beta}\right) \\
V & \rightarrow V_{2} \beta
\end{aligned}
$$

so that

$$
V \int \frac{d^{3} p}{(2 \pi)^{3}} \rightarrow V_{2} \int \frac{d^{2} p}{(2 \pi)^{2}} \sum_{n}
$$

In particular the Euclidean action is given by

$$
S=N V_{2} \sum_{n} \int \frac{d^{2} q}{(2 \pi)^{2}} \operatorname{Tr}\left[\ln \left[i \gamma^{\mu} q_{\mu}+\Sigma_{T}(q)\right]-\frac{1}{2} \Sigma_{T}(q)\left(\frac{1}{i \gamma^{\mu} q_{\mu}+\Sigma_{T}(q)}\right)\right]
$$

where $T$, the temperature is $\beta^{-1}$ and the function $\Sigma_{T}(q)$ obeys the gap equation

$$
\Sigma_{T}(p)=-2 \pi i \lambda \frac{1}{\beta} \sum_{n} \int \frac{d^{2} q}{(2 \pi)^{2}}\left(\gamma^{3} \frac{1}{i \gamma^{\mu} q_{\mu}+\Sigma_{T}(q)} \gamma^{+}-\gamma^{+} \frac{1}{i \gamma^{\mu} q_{\mu}+\Sigma_{T}(q)} \gamma^{3}\right) \frac{1}{(p-q)^{+}}
$$

where $n$ is an integer and

$$
q^{3}=\frac{2 \pi\left(n+\frac{1}{2}\right)}{\beta} .
$$


In order to determine the free energy at finite temperature $T$, we need to solve the gap equation 2.53) and plug the solution into 2.52. We take up these exercises in turn.

\subsubsection{The finite temperature gap equation}

As in subsection 2.1.1 it follows immediately that $\Sigma_{T}$ is a linear combination of $\gamma^{+}$ and $I$, and that it is independent of $p_{3}$. Rotational symmetry and the constraints of conformality then imply

$$
\Sigma_{T}(p)+M_{\text {bare }} I=f\left(\beta p_{s}\right) p_{s} I+i g\left(\beta p_{s}\right) p^{-} \gamma^{+}
$$

for some as yet unknown functions $f\left(\beta p_{s}\right)$ and $g\left(\beta p_{s}\right)$. Note that the new dimensionful scale $\beta$, now allows $f$ and $g$ to be functions of $p_{s}$, generalizing the pure numbers $f_{0}$ and $g_{0}$ of the previous section. The zero temperature results of the previous subsection imply that

$$
\begin{aligned}
& \lim _{y \rightarrow \infty} f(y)=f_{0}=\lambda \\
& \lim _{y \rightarrow \infty} g(y)=g_{0}=-\lambda^{2}
\end{aligned}
$$

The analogue of 2.11 is

$$
\begin{gathered}
p_{s} f\left(p_{s} \beta\right)-M_{\text {bare }}=4 \pi \frac{\lambda}{\beta} \int \sum_{n} \frac{d^{2+\epsilon} q}{(2 \pi)^{2}} \frac{q^{+}}{\left(\frac{2 \pi\left(n+\frac{1}{2}\right)}{\beta}\right)^{2}+q_{s}^{2}\left(1+g\left(q_{s} \beta\right)+\left|f\left(q_{s} \beta\right)\right|^{2}\right)} \frac{1}{(p-q)^{+}} \\
g\left(p_{s} \beta\right) p^{-}=-4 \pi \frac{\lambda}{\beta} \int \sum_{n} \frac{d^{2+\epsilon} q}{(2 \pi)^{2}} q_{s} \frac{f\left(q_{S} \beta\right)}{\left(\frac{2 \pi\left(n+\frac{1}{2}\right)}{\beta}\right)^{2}+q_{s}^{2}\left(1+g+f^{2}\left(q_{s} \beta\right)\right)} \frac{1}{(p-q)^{+}} \quad(2.56)
\end{gathered}
$$

The summations in these equations are easily carried out using the formula

$$
\int d q^{\epsilon} \sum_{n=-\infty}^{\infty} \frac{1}{\left(n+\frac{1}{2}\right)^{2}+a^{2}+q^{2}}=\frac{\pi}{|a|^{1-\epsilon}} \tanh (\pi|a|)
$$

(this is the analogue of 2.15 in the previous section - as in the previous section we have set $\epsilon$ to zero in every place where it will be inessential for regularization) yielding

$$
\begin{gathered}
f p_{s}-M_{\text {bare }}=2 \pi \lambda \int \frac{d^{2} q q_{s}^{-\epsilon}}{(2 \pi)^{2}} \tanh \left(\frac{\beta q_{s}}{2} \sqrt{1+g+f^{2}}\right) \frac{q^{+}}{q_{s}(p-q)^{+} \sqrt{1+g+f^{2}}} \\
g p^{-}=-2 \pi \lambda \int \frac{d^{2} q q^{-\epsilon}}{(2 \pi)^{2}} \tanh \left(\frac{\beta q_{s}}{2} \sqrt{1+g+f^{2}}\right) \frac{f}{\sqrt{1+g+f^{2}}} \frac{1}{(p-q)^{+}}
\end{gathered}
$$


where we have left implicit the fact that the $f$ and $g$ are functions of $p_{s}$ on the LHS of 2.58 2.59), but are functions of $q_{s}$ on the RHS of the same equations.

In each of 2.58) and 2.59) we move to polar coordinates and use use (2.18) to perform the angular integrals to obtain

$$
\begin{aligned}
& f p_{s}=-\lambda \int_{p}^{\infty} q^{-\epsilon} d q_{s} \tanh \left(\frac{\beta q}{2} \sqrt{1+g+f^{2}}\right) \frac{1}{\sqrt{1+g+f^{2}}} \\
& g=-2 \lambda \int_{0}^{p} \frac{q_{s}^{1-\epsilon} d q_{s}}{p_{s}^{2}} \tanh \left(\frac{\beta q_{s}}{2} \sqrt{1+g+f^{2}}\right) \frac{f}{\sqrt{1+g+f^{2}}}
\end{aligned}
$$

Adding and subtracting $q^{-\epsilon}$ from the integrand of 2.60 and doing the integral on the trivial piece we find

$$
\begin{gathered}
f=\lambda-\lambda \int_{p}^{\infty} \frac{d q_{s}}{p_{s}}\left(\frac{\tanh \left(\frac{\beta q_{s}}{2} \sqrt{1+g+f^{2}}\right)}{\sqrt{1+g+f^{2}}}-1\right) \\
g=-2 \lambda \int_{0}^{p} \frac{q_{s} d q_{s}}{p_{s}^{2}} \frac{\tanh \left(\frac{\beta q_{s}}{2} \sqrt{1+g+f^{2}}\right) f}{\sqrt{1+g+f^{2}}}
\end{gathered}
$$

In terms of the variable

$$
\begin{gathered}
x=\frac{q}{p} \\
f(y)=\lambda-\lambda \int_{1}^{\infty} d x\left(\frac{\tanh \left(\frac{y x}{2} \sqrt{1+g(y x)+f(y x)^{2}}\right)}{\sqrt{1+g(y x)+f(y x)^{2}}}-1\right) \\
g(y)=-2 \lambda \int_{0}^{1} x d x\left(\frac{\tanh \left(\frac{y x}{2} \sqrt{1+g(y x)+f(y x)^{2}}\right) f(y x)}{\sqrt{1+g(y x)+f(y x)^{2}}}\right)
\end{gathered}
$$

where the variable

$$
y=p \beta .
$$

Equivalently

$$
\begin{aligned}
& f(y)=\lambda-\frac{\lambda}{y} \int_{y}^{\infty} d x\left(\frac{\tanh \left(\frac{x}{2} \sqrt{1+g(x)+f(x)^{2}}\right)}{\sqrt{1+g(x)+f(x)^{2}}}-1\right) \\
& g(y)=-2 \frac{\lambda}{y^{2}} \int_{0}^{y} x d x \frac{\tanh \left(\frac{x}{2} \sqrt{1+g(x)+f(x)^{2}}\right) f(x)}{\sqrt{1+g(x)+f(x)^{2}}}
\end{aligned}
$$




\subsubsection{The exact solution}

Quite remarkably it is possible to find the exact solution to 2.66). We start with 2.66 written in the form

$$
\begin{gathered}
y\left(f(y)-f_{0}\right)=-\lambda \int_{y}^{\infty} d x\left(\frac{\tanh \left(\frac{x}{2} \sqrt{1+g(x)+f(x)^{2}}\right)}{\sqrt{1+g(x)+f(x)^{2}}}-1\right) \\
y^{2} g(y)=-2 \lambda \int_{0}^{y} x d x \frac{\tanh \left(\frac{x}{2} \sqrt{1+g(x)+f(x)^{2}}\right) f(x)}{\sqrt{1+g(x)+f(x)^{2}}} .
\end{gathered}
$$

Differentiating both equations w.r.t. $y$ we obtain

$$
\begin{aligned}
& y f^{\prime}(y)+f(y)=\lambda \frac{\tanh \left(\frac{y}{2} \sqrt{1+g(y)+f(y)^{2}}\right)}{\sqrt{1+g+f^{2}}} \\
& y g^{\prime}(y)+2 g(y)=-2 \lambda f(y) \frac{\tanh \left(\frac{y}{2} \sqrt{1+g(y)+f(y)^{2}}\right)}{\sqrt{1+g+f^{2}}} .
\end{aligned}
$$

Multiplying the first equation by $2 f$ and add it to the second equation, we cancel the RHS and obtain

$$
y \frac{d}{d y}\left(g+f^{2}\right)+2\left(g+f^{2}\right)=0
$$

From this we solve

$$
g(y)+f(y)^{2}=\frac{c}{y^{2}}
$$

where $c$ is a constant. Now the first equation in 2.68 becomes simply

$$
y f^{\prime}(y)+f(y)=\lambda \frac{\tanh \left(\frac{y}{2} \sqrt{1+\frac{c}{y^{2}}}\right)}{\sqrt{1+\frac{c}{y^{2}}}}
$$

Integrating we have

$$
\begin{aligned}
& f(y)=\frac{\lambda}{y} \int_{0}^{y} d z \frac{\tanh \left(\frac{z}{2} \sqrt{1+\frac{c}{z^{2}}}\right)}{\sqrt{1+\frac{c}{z^{2}}}}+\frac{\tilde{c}}{y}=\frac{2 \lambda}{y} \ln \left(\frac{\cosh \left[\frac{1}{2} \sqrt{c+y^{2}}\right]}{\cosh \left[\frac{\sqrt{c}}{2}\right]}\right)+\frac{\tilde{c}}{y} \\
& g(y)=\frac{c}{y^{2}}-f(y)^{2} .
\end{aligned}
$$

Here $\tilde{c}$ is another integration constant.

While the solution to a differential equation depends on integration constants, the solution to an integral equation is unique (it does not have undetermined integration 
constants). The appearance of $c$ and $\tilde{c}$ in our solutions above is an artifact of our having solved by converting the integral equation into a differential equation. The integral equations 2.66$)$ are actually solved by 2.72 only for a particular choice of $c$ and $\tilde{c}$.

We first note that the function $f$ must tend to $f_{0}$ at large $y$. This is automatic in all our solutions (it does not impose any constraints on $c$ or $\tilde{c}$ ). However a further requirement is that the expansion of $f$ about this constant value (at large $y$ ) should start at $\frac{1}{y^{2}}$ rather than $\frac{1}{y}$ (this follows immediately upon plugging (2.70) into the RHS of the first of (2.67); the RHS of that equation is manifestly $\propto \frac{1}{y}$ ). The requirement that

$$
f(y)=f_{0}+\mathcal{O}\left(1 / y^{2}\right)
$$

determines

$$
\tilde{c}=2 \lambda \ln \left(2 \cosh \frac{\sqrt{c}}{2}\right) .
$$

Let us now turn to the small $y$ behaviour of $f$ and $g$. At small $y$

$$
\begin{aligned}
f(y) & =\frac{\tilde{c}}{y}+\mathcal{O}(y), \\
g(y) & =\frac{c-\tilde{c}^{2}}{y^{2}}+\mathcal{O}\left(y^{0}\right) .
\end{aligned}
$$

Plugging 2.70 into the RHS of the second of 2.67), however, we find that the RHS evaluates to $\mathcal{O}\left(y^{2}\right)$ at small $y$ implying that $g(y)=\mathcal{O}\left(y^{0}\right)$ at small $y$. It follows that

$$
c=\tilde{c}^{2}
$$

Plugging this relation into 2.74 yields the following equation for $\tilde{c}$

$$
\frac{\tilde{c}}{2 \lambda}=\ln \left(2 \cosh \frac{\tilde{c}}{2}\right) \text {. }
$$

Note that $\tilde{c}$ is an odd function of $\lambda .|\tilde{c}|$ is a monotonically increasing function of $\lambda$, which diverges at $|\lambda|=1$. 2.77) has no solution for $|\lambda|>1$, indicating that the theory does not exist for $|k|>N$. At leading order in at small $\lambda$ we have

$$
\tilde{c}=2 \lambda \ln 2+\mathcal{O}\left(\lambda^{3}\right)
$$

As $\lambda$ approaches unity we have

$$
\tilde{c}=\ln \frac{2}{(1-\lambda)}-\ln \left(\ln \frac{2}{1-\lambda}\right)+\mathcal{O}\left(\ln \ln \ln \frac{2}{1-\lambda}\right) .
$$


In order to physically interpret the divergence of $\tilde{c}$ as $\lambda \rightarrow 1$ note that the exact thermal propagator has a pole whenever

$$
p^{2}+\tilde{c}^{2} T^{2}=0
$$

In other words $\tilde{c}$ has a simple physical interpretation; it is the thermal mass of the field $\psi$ in units of the temperature. It follows that the fermion thermal mass diverges in this limit $\lambda \rightarrow 1$.

Using (2.77) and (2.76) we may rewrite our solutions for $f$ and $g$ as

$$
\begin{aligned}
& f(y)=\frac{2 \lambda}{y} \ln \left(2 \cosh \frac{\sqrt{\tilde{c}^{2}+y^{2}}}{2}\right), \\
& g(y)=\frac{\tilde{c}^{2}}{y^{2}}-f(y)^{2} .
\end{aligned}
$$

with $\tilde{c}$ given by $(2.77)$. In the large $y$ limit

$$
\begin{aligned}
& f(y)=\lambda \sqrt{1+\frac{\tilde{c}^{2}}{y^{2}}+\mathcal{O}\left(e^{-y}\right),} \\
& g(y)=-\lambda^{2}+\frac{\tilde{c}^{2}\left(1-\lambda^{2}\right)}{y^{2}}+\mathcal{O}\left(e^{-y}\right),
\end{aligned}
$$

while at small $y$

$$
\begin{aligned}
& f(y)=\frac{\tilde{c}}{y}+\frac{\lambda y}{2 \tilde{c}} \tanh \left(\frac{\tilde{c}}{2}\right)+\mathcal{O}\left(y^{3}\right), \\
& g(y)=-\lambda \tanh \left(\frac{\tilde{c}}{2}\right)+\mathcal{O}\left(y^{2}\right) .
\end{aligned}
$$

\subsubsection{Free energy as a function of temperature}

As we have explained above, the path integral of our theory on the manifold $\mathbb{R}^{2} \times S^{1}$, with the circumference of the $S^{1}$ equal to $\beta$, is given by $e^{-S_{T}}$ where

$$
S_{T}=N V_{2} \sum_{n} \int \frac{d^{2} q}{(2 \pi)^{2}} \operatorname{Tr}\left[\ln \left[i \gamma^{\mu} q_{\mu}+\Sigma_{T}(q)\right]-\frac{1}{2} \Sigma_{T}(q)\left(\frac{1}{i \gamma^{\mu} q_{\mu}+\Sigma_{T}(q)}\right)\right]
$$

Let us define

$$
S_{0}=-N V_{2} \beta \int \frac{d^{3} q}{(2 \pi)^{3}} \operatorname{Tr}\left[\ln \left[i \gamma^{\mu} q_{\mu}+\Sigma(q)\right]-\frac{1}{2} \Sigma(q)\left(\frac{1}{i \gamma^{\mu} q_{\mu}+\Sigma(q)}\right)\right] .
$$

Then the partition function

$$
Z=\operatorname{Tr} e^{-\beta H}
$$


of our system in a flat spatial box of volume $V_{2}$ is given by

$$
\ln Z=S_{0}-S_{T},
$$

so that the finite temperature free energy, $F(T)$, of the theory is given by

$$
F(T)=\frac{S_{T}-S_{0}}{\beta}
$$

We will now proceed to use our exact solution to the finite temperature gap equation to compute $S_{T}-S_{0}$.

\subsubsection{Explicit evaluation of the free energy}

In order to find an explicit expression for the free energy, we find it convenient to use the expressions in the second line of 2.83$)$ and (2.84). $S_{T}-S_{0}$ may be written as

$$
\begin{aligned}
& S_{T}-S_{0}=-N V_{2} \sum_{n} \int \frac{d^{2} q}{(2 \pi)^{2}} \operatorname{Tr} \ln \left(i \gamma^{\mu} q_{\mu}+\Sigma_{T}(q)\right)+N V_{2} \int \frac{d^{3} q}{(2 \pi)^{3}} \operatorname{Tr} \ln \left(i \gamma^{\mu} q_{\mu}+\Sigma_{T}(q)\right) \\
& -N V_{2} \beta \int \frac{d^{3} q}{(2 \pi)^{3}} \operatorname{Tr} \ln \frac{\left(i \gamma^{\mu} q_{\mu}+\Sigma_{T}(q)\right)}{\left(i \gamma^{\mu} q_{\mu}+\Sigma(q)\right)} \\
& +\frac{N V_{2}}{2} \sum_{n} \int \frac{d^{2} q}{(2 \pi)^{2}} \operatorname{Tr}\left(\frac{q_{s} f\left(\beta q_{s}\right)+i g\left(\beta q_{s}\right) q_{+} \gamma^{+}}{i \gamma^{\mu} q_{\mu}+\Sigma_{T}(q)}\right)-\frac{N V_{2} \beta}{2} \int \frac{d^{3} q}{(2 \pi)^{3}} \operatorname{Tr}\left(\frac{f_{0} q_{s}+i g_{0} p_{+} \gamma^{+}}{i \gamma^{\mu} q_{\mu}+\Sigma(q)}\right) .
\end{aligned}
$$

The integral on the first line of 2.85 is convergent and evaluates to

$$
\begin{aligned}
& -N V_{2} \sum_{n} \int \frac{d^{2} q}{(2 \pi)^{2}} \ln \left(q_{3}^{2}+q_{s}^{2}+\tilde{c}^{2} T^{2}\right)+N V_{2} \beta \int \frac{d^{3} q}{(2 \pi)^{3}} \ln \left(q_{3}^{2}+q_{s}^{2}+\tilde{c}^{2} T^{2}\right) \\
& =-2 N V_{2} \int \frac{d^{2} q}{(2 \pi)^{2}} \ln \left(1+e^{-\beta \sqrt{\tilde{c}^{2} T^{2}+q_{s}^{2}}}\right) \\
& =-2 N T^{2} V_{2} \int \frac{d^{2} x}{(2 \pi)^{2}} \ln \left(1+e^{-\sqrt{\tilde{c}^{2}+x_{s}^{2}}}\right) \\
& =-\frac{2 N T^{2} V_{2}}{2 \pi} \int_{|\tilde{c}|}^{\infty} d y y \ln \left(1+e^{-y}\right) .
\end{aligned}
$$

The second line of 2.85 has a linear divergence, which however disappears in our dimensional reduction scheme

$$
\begin{aligned}
& -N V_{2} \beta \int \frac{d^{3-\epsilon} q}{(2 \pi)^{3}} \ln \frac{\left(q^{2}+\tilde{c}^{2} T^{2}\right)}{\left(q^{2}\right)} \\
& =-N V_{2} T^{2}|\tilde{c}|^{3} \int \frac{d^{3-\epsilon} y}{(2 \pi)^{3}} \ln \frac{y^{2}+1}{y^{2}} \\
& =-\frac{N V_{2} T^{2}|\tilde{c}|^{3}}{2 \pi^{2}} \int_{0}^{\infty} d y y^{2-\epsilon} \ln \left(1+\frac{1}{y^{2}}\right)=\frac{N V_{2} T^{2}|\tilde{c}|^{3}}{6 \pi}
\end{aligned}
$$


where in the last step we integrated by parts and used

$$
\int_{0}^{\infty} \frac{y^{2-\epsilon}}{1+y^{2}}=-\frac{\pi}{2}
$$

in the limit of small $\epsilon$. Let us now turn to the third line of 2.85 . The second term in the third line simply vanishes under dimensional regularization. The first term in the third line may be evaluated as follows

$$
\begin{aligned}
& \frac{N V_{2}}{2} \sum_{n} \int \frac{d^{2} q}{(2 \pi)^{2}} \frac{2 q_{s}^{2} f^{2}\left(\beta q_{s}\right)+q_{s}^{2} g\left(\beta q_{s}\right)}{q_{3}^{2}+q_{s}^{2}+T^{2} \tilde{c}^{2}} \\
& =\frac{N V_{2}}{4} \int \frac{d^{2} q}{(2 \pi)^{2}} \frac{\left(2 q_{s}^{2} f^{2}\left(\beta q_{s}\right)+q_{s}^{2} g\left(\beta q_{s}\right)\right) \tanh \frac{\sqrt{q^{2}+\tilde{c}^{2}}}{2}}{\sqrt{q_{s}^{2}+T^{2} \tilde{c}^{2}}} \\
& =\frac{N V_{2} T^{2}}{4}\left[\int \frac{d^{2} q}{(2 \pi)^{2}} \frac{\left(2 q_{s}^{2} f^{2}\left(q_{s}\right)+q_{s}^{2} g\left(q_{s}\right)\right) \tanh \frac{\sqrt{q^{2}+\tilde{c}^{2}}}{2}}{\sqrt{q_{s}^{2}+\tilde{c}^{2}}}-\lambda^{2}\left(\tilde{c}^{2}+q^{2}\right)-\tilde{c}^{2}\right. \\
& \left.\quad+\int \frac{d^{2} q}{(2 \pi)^{2}} \frac{\lambda^{2}\left(\tilde{c}^{2}+q^{2}\right)+\tilde{c}^{2}}{\sqrt{q_{s}^{2}+\tilde{c}^{2}}}\right] .
\end{aligned}
$$

The first term in 2.88 is finite and evaluates to

$$
\frac{N V_{2} T^{2}}{4 \pi}|\tilde{c}|^{3}\left(\frac{\lambda^{2}}{6}-\frac{1}{6|\lambda|}-\frac{1}{2|\lambda|}+\frac{1}{2}\right) .
$$

The second term in 2.88 is divergent. In dimensional regularization it evaluates to

$$
-\frac{N V_{2} T^{2}|\tilde{c}|^{3}}{4 \pi}\left(\frac{\lambda^{2}}{6}+\frac{1}{2}\right) \text {. }
$$

The third line of 2.85 is given by the sum of 2.89 and 2.90 and evaluates to

$$
-\frac{N V_{2} T^{2}|\tilde{c}|^{3}}{6 \pi|\lambda|}
$$

Putting it all together we find that the free energy is given by

$$
F=-\frac{N V_{2} T^{3}}{6 \pi}\left[|\tilde{c}|^{3} \frac{1-|\lambda|}{|\lambda|}+6 \int_{|\tilde{c}|}^{\infty} d y y \ln \left(1+e^{-y}\right)\right] .
$$

Although it is not manifest, the free energy can also be written in the form (1.1), and is an analytic function of $\lambda$ in the interval $(-1,1){ }^{11}$ To see this more explicitly, note

\footnotetext{
${ }^{11}$ Theories with massless bosons usually do not have an analytic expansion of their free energy in terms of the coupling constant. Such non-analytic behavior in Chern-Simons-matter theories with massless bosons was observed explicitly e.g. in 7], 38. This non-analyticity has its origin in infrared divergences which are absent in our theory because the only propagating degrees of freedom are the fermions which lack a zero-mode along the thermal circle.
} 


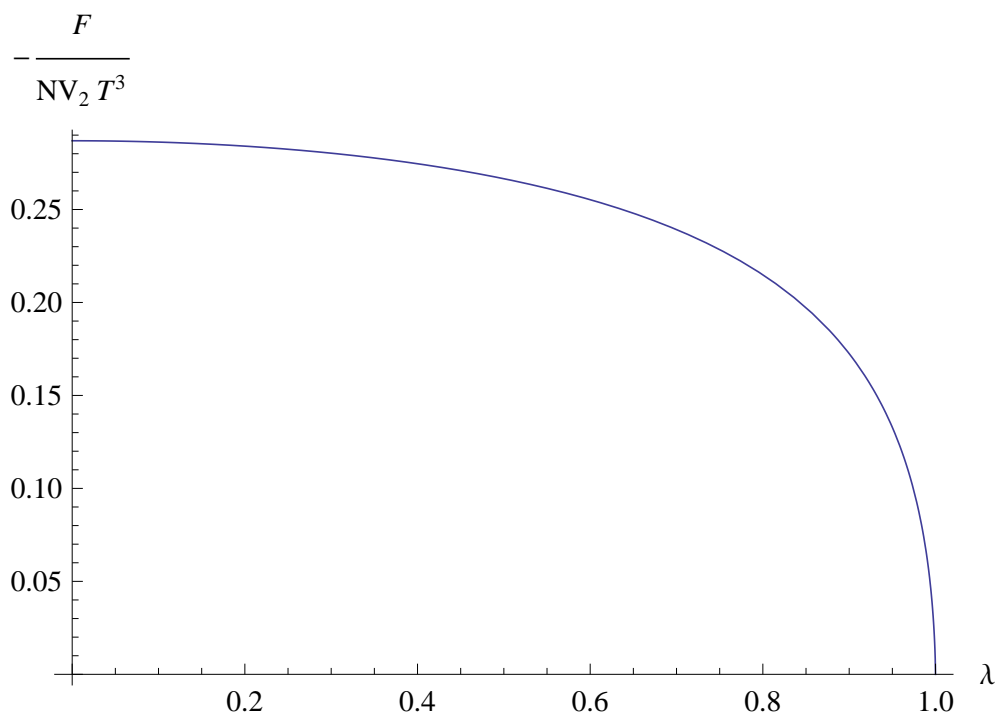

Figure 1: The free energy on $S^{1} \times \mathbb{R}^{2}$ as a function of $\lambda$.

that we can write the term in square brackets in 2.92 as

$$
\begin{aligned}
& \frac{|\tilde{c}|^{3}}{|\lambda|}-|\tilde{c}|^{3}+6 \int_{0}^{\infty} d y y \ln \left(1+e^{-y}\right)+6 \int_{0}^{|\tilde{c}|} d y\left(\frac{y^{2}}{2}-y \ln \left(2 \cosh \frac{y}{2}\right)\right) \\
& =\frac{9}{2} \zeta(3)+\frac{\tilde{c}^{3}}{\lambda}-6 \int_{0}^{|\tilde{c}|} d y y \ln \left(2 \cosh \frac{y}{2}\right),
\end{aligned}
$$

where in the last line we used $|\tilde{c}|^{3} /|\lambda|=\tilde{c}^{3} / \lambda$, as follows from (2.77). Note that the integral in the last term is clearly an even function of $|\tilde{c}|$, and so the absolute value can be omitted. This shows that one may effectively rewrite (2.92) as in (1.1), which is manifestly analytic. Indeed its small $\lambda$ expansion is given by

$$
F=-N V_{2} T^{3}\left[\frac{3 \zeta(3)}{4 \pi}-\frac{2(\log 2)^{3}}{3 \pi} \lambda^{2}-\frac{(\log 2)^{4}}{2 \pi} \lambda^{4}+\mathcal{O}\left(\lambda^{6}\right)\right]
$$

Note that it contains only even powers of $\lambda$, consistently with parity. A plot of the free energy as a function of $\lambda$ is given in Fig. 1. We see that $-F$ decreases monotonically from the free field value $-F=\frac{3 N V_{2} T^{3}}{4 \pi} \zeta(3)$ to zero at $\lambda=1$.

In the limit that $|\lambda| \rightarrow 1,|\tilde{c}|$ is large and the integral in 2.92 may be approximated by

$$
\int_{|\tilde{c}|}^{\infty} d y y \ln \left(1+e^{-y}\right)=|\tilde{c}| e^{-|\tilde{c}|}+e^{-|\tilde{c}|}+\mathcal{O}\left(e^{-2 \tilde{c} \mid}\right) .
$$

At leading nontrivial order this evaluates to

$$
\tilde{c}^{2} \frac{1-|\lambda|}{2},
$$


and the free energy is given by

$$
F=-\frac{N V_{2} T^{3}(1-|\lambda|)}{6 \pi}\left[|\tilde{c}|^{3}+3 \tilde{c}^{2}+\mathcal{O}(\tilde{c})\right], \quad|\lambda| \rightarrow 1
$$

where $\tilde{c}$ is given by 2.79 .

\subsection{Consistency of our gauge and regularization scheme}

As we have emphasized on multiple occasions, we have obtained the beautiful result (2.92) by employing a rather unusual gauge (a lightcone like gauge in Euclidean space) and the regularization scheme of dimensional reduction, which we have assumed preserves the gauge invariance of our theory. In this section we list the independent evidence that the procedure employed in this section defines a sensible and Lorentz invariant theory.

1. The exact fermion propagator $(2.23)$ develops poles only when $p^{2}=0$, a condition that is Lorentz invariant. This is a necessary condition for the Lorentz invariance of fermion scattering processes in our theory. 12 The Lorentz invariance of the poles of the propagator is far from automatic, and is in fact violated in several regularization schemes. In, for instance, the regularization scheme described just below 2.22 we find $g_{0}=0$ but $f_{0} \neq 0$ (in fact $f_{0}$ obeys the equation $f_{0}=-\frac{\lambda}{\sqrt{1+f_{0}^{2}}}$ with this regulator). The poles of the fermion propagator with this regulator occur at $p_{3}^{2}+p_{s}^{2}\left(1+f_{0}\right)$ and are not Lorentz invariant.

2. In Appendix $\mathrm{C}$ we have demonstrated that the expectation value, at one loop, of the gauge invariant Wilson line operator is Lorentz invariant and moreover agrees with the result obtained in the manifestly Lorentz invariant Feynman gauge.

3. Our results above indicate that our theory is infinitely strongly coupled at $\lambda=1$, and that the theory does not exist (at least as a conformally invariant theory) for $\lambda>1$. There is in fact a very simple interpretation of this result. It is well-known that the bare Chern-Simons level can acquire a finite shift at 1-loop order in perturbation theory. This effect is regularization dependent. It was demonstrated by 21] that the Chern-Simons matter theory defined using the

\footnotetext{
${ }^{12}$ Particle scattering is, strictly speaking, not well defined in our theory (or any conformal theory) due to infrared divergences. We could cure these divergences in our theory softly breaking conformal invariance with a fermion mass. Scattering processes in this deformed theory are presumably well defined. The poles of the mass deformed theory are clearly physical and must be Lorentz invariant. As our computation of the propagator of massless theory, presented in this paper, exhibits no IR divergences, it must equal the zero mass limit of the mass deformed propagator, and hence must be Lorentz invariant.
} 
dimensional reduction scheme, employed in this paper, does not acquire a 1-loop shift of $k$. On the other hand, if the theory is regulated by the addition of a small Yang Mills term, the bare level, which we denote $k_{Y M}$, gets shifted by $\operatorname{sign}\left(k_{Y M}\right) N$. Therefore the results of 21 imply that the two regularizations yield the same physical theory provided

$$
|k|=\left|k_{Y M}\right|+N
$$

Let us define $\lambda=\frac{N}{k}$ as we have done in this paper, and $\left|\lambda_{Y M}\right|=\frac{N}{\left|k_{Y M}\right|}$. It follows that

$$
\left|\lambda_{Y M}\right|=\frac{|\lambda|}{1-|\lambda|}
$$

and

$$
|\lambda|=\frac{\left|\lambda_{Y M}\right|}{1+\left|\lambda_{Y M}\right|}
$$

In particular as $|\lambda| \rightarrow 1$, we have $\left|\lambda_{Y M}\right| \rightarrow \infty$. Now a Chern-Simons theory regulated by the addition of a small Yang Mills term clearly exists at all values of $\left|\lambda_{Y M}\right|$. However it follows from the discussion above that this only requires the dimensionally regulated theory to exist for $|\lambda| \leq 1$. Moreover the limit $|\lambda| \rightarrow 1$ should be interpreted as the approach to strong coupling. Our exact result for the finite temperature free energy is perfectly consistent with this interpretation. The theory ceases to exist for $|\lambda|>1$. Moreover the limit $|\lambda| \rightarrow 1$ displays the extreme thinning of degrees of freedom that is plausible in an extreme strong coupling limit.13

4. In Appendix F.3 we have explicitly computed the two loop anomalous dimension, at first subleading order in $\frac{1}{N}$, of the scalar operator $\bar{\psi} \psi$. The result so obtained agrees perfectly with the result of the same computation performed in Feynman gauge.

The points listed above make it at least plausible that our gauge choice is free of problems, and that our regularization scheme preserves gauge invariance and so defines a Lorentz invariant theory. While the direct evidence for these claims is substantial, it is not overwhelming. Significant additional evidence for the Lorentz invariance of our final theory could be obtained by demonstrating the Lorentz invariance of four Fermi scattering at one loop. We will not, however, attempt that consistency check in this paper.

\footnotetext{
${ }^{13}$ Note that 2.95 expressed in terms of $k_{Y M}$ takes the form

$$
F=-\frac{\left|k_{Y M}\right| V_{2} T^{3}}{6 \pi}\left(\ln \frac{N}{\left|k_{Y M}\right|}\right)^{3}+\cdots, \quad \lambda \rightarrow 1 .
$$

This behavior in the $\lambda \rightarrow 1$ limit could be consistent with a weakly coupled boson description (see 7 ).
} 


\section{Operator Spectrum}

In this section, we study the spectrum of gauge invariant operators in our theory. We focus mainly on operators whose dimension is held fixed as $N$ is taken to infinity. By large $N$ factorization, all such operators are products of "single trace" operators. By a single trace we mean an operator that is constructed from the contraction of a fundamental (derivative of $\psi$ ) and an antifundamental (derivative of $\bar{\psi}$ ). We will argue below that the spectrum of single trace operators in our theory includes one scalar and one current of spin $s$ for $s=1 \ldots \infty$. The dimension of the scalar operator is given, at every value of $\lambda$, by $2+\mathcal{O}(1 / N)$, while the dimension of the spin $s$ current $J^{(s)}$ is given by $\Delta=s+1+\mathcal{O}(1 / N)$. We will also argue that the currents $J^{(s)}$ are "almost" conserved; more precisely that these currents obey the anomalous conservation law listed schematically in (3.16). These nonlinear anomalous conservation equations carry a great deal of information, and may eventually prove very useful in "solving" the theory.

\subsection{The free limit}

In this subsection we review several well known properties of Chern-Simons theory coupled to fundamental fermions in the free $(\lambda \rightarrow 0)$ limit. In this limit our theory reduces to a theory of free fundamental fermions subject to a $U(N)$ singlet Gauss law constraint; this limit has, of course, been studied in the previous literature (see e.g. 28]). In this section we gather those results that will help us study the theory at finite $\lambda$.

\subsection{1 "Single trace" conformal representation content of the free theory}

${ }^{14}$ In this subsection we compute the "single trace" operator content of the theory of free fermions. By "single traces" we mean those operators in our theory that are given by contracting a single (but arbitrary) fundamental field with an anti-fundamental field. The results of this subsection were already presented in [28]; however we rederive them here for completeness.

Let $\Delta$ represent the scaling dimension and $J_{3}$ the $z$ component of the angular momentum of any operator. We will now compute the partition function

$$
\operatorname{Tr} x^{\Delta} \mu^{J_{3}}
$$

\footnotetext{
${ }^{14}$ This subsection was worked out in collaboration with J. Bhattacharya.
} 
over all single trace operators in our theory. As "single traces" are obtained by multiplying an arbitrary fundamental field with an arbitrary anti-fundamental, the trace over "single traces" is simply the product of the partition function for elementary fundamental fields with the partition function for elementary antifundamental fields.

The partition function over elementary fundamental fermionic fields (including arbitrary numbers of derivatives but modulo equations of motion) is simply the character of the $\left(1, \frac{1}{2}\right)$ representation of the conformal group (see Appendix $\mathrm{D}$ and is given by

$$
F_{F}(x, \mu)=\frac{x\left(\mu^{\frac{1}{2}}+\mu^{-\frac{1}{2}}\right)}{(1-\mu x)\left(1-\mu^{-1} x\right)}
$$

The partition function over antifundamentals is given by the same formula, and so the "single trace" partition function is given by $F_{F}^{2}$.

It is not difficult to decompose this partition function into the contribution of primary operators and their descendents. In order to accomplish this we note that

$$
\begin{aligned}
& {\left[\frac{x\left(\mu^{\frac{1}{2}}+\mu^{-\frac{1}{2}}\right)}{(1-\mu x)\left(1-\mu^{-1} x\right)}\right]^{2}} \\
& =\frac{1}{(1-\mu x)\left(1-\mu^{-1} x\right)(1-x)}\left[\frac{x^{2}(1-x)\left(2+\mu+\mu^{-1}\right)}{(1-\mu x)\left(1-\mu^{-1} x\right)}\right] \\
& =\frac{1}{(1-\mu x)\left(1-\mu^{-1} x\right)(1-x)}\left[x^{2}+\sum_{s=1}^{\infty}\left(x^{s+1} \chi_{s}(\mu)-x^{s+2} \chi_{s-1}(\mu)\right)\right] \\
& =\chi_{2,0}(x, \mu)+\sum_{s=1}^{\infty} \chi_{s+1, s}(x, \mu)
\end{aligned}
$$

where $\chi_{\Delta, s}(x, \mu)$ is the character of a representation of the conformal algebra with dimension $\Delta$ and spin $s$ and we have used the character formulae of Appendix $\mathrm{D}$. It follows that single trace operators are given by primaries that transform in the representations

$$
(2,0)+\sum_{j=1}^{\infty}(j+1, j)
$$

together with their descendents. It will be important below that the only long operator that appears in this decomposition is $(2,0)$ (see Appendix $\mathrm{D}$ ); all other operators in the list above appear in short representations of the conformal algebra.

\subsubsection{Explicit form of the primary operators}

As we have explained above, the primary field content of the free fermion theory is given by the $(2,0)$ operator $\bar{\psi} \psi$ plus symmetric traceless currents $J_{\mu_{1} \ldots \mu_{s}}^{(s)}$ for all $s$. As 
we have seen above, the currents are primaries that head short representations of the conformal algebra; the shortening condition is simply the statement that the currents obey the conservation equation

$$
\partial^{\mu} J_{\mu \mu_{1} \ldots \mu_{s-1}}^{(s)}=0
$$

Single trace currents $J^{(s)}$ that have dimension $s+1$, spin $s$ and are conserved are unique up to a choice of scale in the free fermion theory. We have found explicit expressions for each of the currents $J^{(s)}$. Following [31] we find it convenient to package these expressions in the form of a generating function $O(x, \epsilon)$ defined by

$$
\mathcal{O}(x ; \epsilon)=\sum J_{\mu_{1} \mu_{2} \ldots \mu_{s}}^{(s)} \epsilon^{\mu_{1}} \ldots \epsilon^{\mu_{s}}
$$

where $\epsilon^{\mu}$ is an arbitrary vector. As all currents are bilinear in the fermions, the generating function is given by an expression of the form

$$
\mathcal{O}(x ; \epsilon)=\bar{\psi} F\left(\vec{\gamma}, \overrightarrow{\partial_{\mu}}, \overleftarrow{\partial_{\mu}}, \vec{\epsilon}\right) \psi
$$

Making a convenient choice for the overall scale of each $J^{(s)}$ we find

$$
F=\vec{\gamma} \cdot \vec{\epsilon} f\left(\vec{\partial}_{\mu}, \overleftarrow{\partial_{\mu}}, \vec{\epsilon}\right)
$$

where

$$
f(\vec{u}, \vec{v}, \vec{\epsilon})=\frac{\exp (\vec{u} \cdot \vec{\epsilon}-\vec{v} \cdot \vec{\epsilon}) \sinh \sqrt{2 \vec{u} \cdot \vec{v} \vec{\epsilon} \cdot \vec{\epsilon}-4 \vec{u} \cdot \vec{\epsilon} \vec{v} \cdot \vec{\epsilon}}}{\sqrt{2 \vec{u} \cdot \vec{v} \vec{\epsilon} \cdot \vec{\epsilon}-4 \vec{u} \cdot \vec{\epsilon} \cdot \vec{\epsilon}}}
$$

The Taylor expansion

$$
\begin{aligned}
f= & 1+\epsilon(u-v)+\frac{1}{6} \epsilon^{2}\left(3 u^{2}-10 u v+3 v^{2}+2 w\right) \\
& +\epsilon^{3}\left(\frac{u^{3}}{6}-\frac{7 u^{2} v}{6}+\frac{7 u v^{2}}{6}+\frac{u w}{3}-\frac{v^{3}}{6}-\frac{v w}{3}\right) \\
& +\frac{1}{120} \epsilon^{4}\left(10(u-v)^{2}(2 w-4 u v)+(4 u v-2 w)^{2}+5(u-v)^{4}\right)+\mathcal{O}\left(\epsilon^{5}\right)
\end{aligned}
$$

(above, $w=\vec{u} \cdot \vec{v}, u=\vec{u} \cdot \vec{\epsilon}, v=\vec{v} \cdot \vec{\epsilon}$.) implies the following explicit expressions for the first four currents

$$
\begin{aligned}
J_{\mu}= & \bar{\psi} \gamma_{\mu} \psi \\
J_{\mu_{1} \mu_{2}}= & \bar{\psi} \gamma_{\mu_{1}}\left(\overrightarrow{\partial_{\mu_{2}}}-\overleftarrow{\partial_{\mu_{2}}}\right) \psi \\
J_{\mu_{1} \mu_{2} \mu_{3}}= & \frac{1}{6} \bar{\psi} \gamma_{\mu_{1}}\left(3 \overleftarrow{\partial_{\mu_{2}}} \overleftarrow{\partial_{\mu_{3}}}-10 \overleftarrow{\partial_{\mu_{2}}} \overrightarrow{\partial_{\mu_{3}}}+3 \overrightarrow{\partial_{\mu_{2}}} \overrightarrow{\partial_{\mu_{3}}}+2\left(\overleftarrow{\partial_{\sigma}} \overrightarrow{\partial^{\sigma}}\right) \eta_{\mu_{2} \mu_{3}}\right) \psi \\
J_{\mu_{1} \mu_{2} \mu_{3} \mu_{4}}= & \frac{1}{6} \bar{\psi} \gamma_{\mu_{1}}\left(\overleftarrow{\partial_{\mu_{2}}} \overleftarrow{\partial_{\mu_{3}}} \overleftarrow{\partial_{\mu_{4}}}-7 \overleftarrow{\partial_{\mu_{2}}} \overleftarrow{\partial_{\mu_{3}}} \overrightarrow{\partial_{\mu_{4}}}+7 \overleftarrow{\partial_{\mu_{2}}} \overrightarrow{\partial_{\mu_{3}}} \overrightarrow{\partial_{\mu_{4}}}-\overrightarrow{\partial_{\mu_{2}}} \overrightarrow{\partial_{\mu_{3}}} \overrightarrow{\partial_{\mu_{4}}}\right. \\
& \left.+2\left(\overleftarrow{\partial_{\sigma}} \overrightarrow{\partial^{\sigma}}\right) \overleftarrow{\partial_{\mu_{2}}} \eta_{\mu_{3} \mu_{4}}-2\left(\overleftarrow{\partial_{\sigma}} \overrightarrow{\partial^{\sigma}}\right) \overrightarrow{\partial_{\mu_{2}}} \eta_{\mu_{3} \mu_{4}}\right) \psi
\end{aligned}
$$

where all indices above are understood to be symmetrized.

The existence of spin $s$ conserved currents gives rise to a large symmetry algebra, called the higher spin algebra, of the free fermion theory. 


\subsubsection{The full multitrace partition function}

In this section we present a computation of the partition function of the free theory on $S^{2} \times S^{1}$, i.e. the free theory on $S^{2}$ at finite temperature, along the lines of [39]. By the state operator map this is the same as the partition function over all operators (single and multi trace) of the free theory.

The exact partition function on $S^{2} \times S^{1}$ is given by 41,40

$$
Z=\int D U \exp \left(-\sum_{n} \frac{N^{2}\left|\rho_{n}\right|^{2}-N(-1)^{n+1} F_{F}\left(x^{n}\right)\left(\rho_{n}+\rho_{n}^{*}\right)}{n}\right)
$$

where $x=e^{-\beta}=e^{-\frac{1}{T}}, F(x)$ is the letter partition function of the fundamental fermions, $U$ is a unitary matrix (the holonomy of $A_{\mu}$ around the time circle) and

$$
\rho_{n}=\frac{1}{N} \operatorname{Tr} U^{n}
$$

Let us first study $(3.9)$ in the limit that $x$ is held fixed as we take the large $N$ limit. In this case the integral (3.9) is very simply evaluated by completing the square in the $\rho_{n}$ variables and yields

$$
Z=e^{\sum_{n} \frac{F_{F}\left(x^{n}\right)^{2}}{n}}
$$

In the high temperature limit $F_{F}(x)=2 T^{2}$ and 3.10 reduces approximately to

$$
\ln Z=4 T^{4} \zeta(5)
$$

(3.10) is simply the Bose exponentiation the single particle partition function $F_{F}(x)^{2}$. However we have already argued above that $F(x)^{2}$ is precisely the single trace operator partition function. It follows that 3.10 represents the partition function of a noninteracting gas of single trace operators (or particles), with single trace partition function $F_{F}(x)^{2}$.

In order to obtain the result 3.10 we shifted the variable $\rho_{n}$ by $\frac{F\left(x^{n}\right)}{N}$. When $x$ is of order unity this shift is of order $\frac{1}{N}$ and is negligible. In the high temperature limit, however,

$$
F_{F}(x) \approx 2 T^{2}
$$

and the large $N$ saddle point value of the integral 3.10$)$ is given by

$$
\rho_{n}=(-1)^{n+1} \frac{2 T^{2}}{N n^{2}}
$$

If we set

$$
T=\sqrt{N} t
$$


and hold $t$ fixed as $N$ is sent to $\infty$, the saddle point value of $\rho_{n}$ is independent of $N$

$$
\rho_{n}=(-1)^{n} \frac{2 t^{2}}{n^{2}}
$$

Let $\rho(\theta)$ denote the density of eigenvalues of the unitary matrix $U$. It follows that

$$
\rho_{n}=\frac{1}{N} \operatorname{Tr} U^{n}=\int d \theta \rho(\theta) e^{i n \theta}
$$

The saddle point eigenvalue distribution may be reconstructed from its Fourier modes via

$$
\rho(\theta)=\frac{1}{2 \pi}\left[1+\sum_{n=1}^{\infty}\left(\rho_{-n} e^{i n \theta}+\rho_{n} e^{-i n \theta}\right)\right]
$$

The eigenvalue distribution that corresponds, in particular, to 3.12 is given by

$$
2 \pi \rho(\theta)=1+4 t^{2} \sum_{n=1}^{\infty} \frac{(-1)^{n+1}}{n^{2}} \cos n \theta .
$$

This eigenvalue distribution takes its minimum value at $\theta=\pi . \rho(\pi)$ is negative when

$$
t \geq \frac{\sqrt{3}}{\sqrt{2} \pi}
$$

Now a negative value for the eigenvalue density function is meaningless; when the inequality (3.13) is obeyed what really happens is that our system undergoes a GrossWitten-Wadia phase transition [29,30] to a gapped phase.

The saddle point solution for $\rho(\theta)$ is quantitatively complicated above the phase transition temperature (3.13). In broad qualitative terms, however, the eigenvalue distribution gets increasingly peaked as $t$ increases. In the limit $t \gg 1$ the eigenvalue distribution tends to a $\delta$ function. In this limit $\rho_{n}=1$ for all $n$, and the logarithm of the partition function is given by

$$
\ln Z=4 N T^{2} \sum_{n=1}^{\infty} \frac{(-1)^{n+1}}{n^{3}}=3 N \zeta(3) T^{2}
$$

In this limit we recover the partition function of the free theory on $\mathbb{R}^{2}$ (the leading term in (2.94), with the volume factor $V_{2}$ replaced by $4 \pi$, the volume of the unit two sphere.

In summary, the partition function of our theory on an $S^{2}$ of unit radius interpolates between the partition function of a gas over single trace operators 3.10 and 3.11) (when $T \ll \sqrt{N}$ ) to the gas of deconfined fermions (when $t \gg \sqrt{N}$ ). Note that when $\ln Z$ is of order $N^{2}$ when $T$ approaches $\sqrt{N}$ both from below (see (3.11)) and from above (see (3.14)). The interpolation between these behaviours is not smooth; it goes through a third order Gross-Witten-Wadia transition. 


\subsection{Non-renormalization of the scaling dimension of the cur- rent operators}

As we have explained above, the single trace operator content, in the free limit, is given in terms of representations of the conformal algebra by

$$
(0,2)+\sum_{j=1}^{\infty}(j+1, j)
$$

As we have explained in Appendix D, representations of the sort $(s+1, s)$ are short. Operators that transform in these representations can develop anomalous dimensions only after combining with a long representation with quantum numbers $(s+2, s-1)$ (see (D.5)).

Now the operators in the $(2,1)$ and $(3,2)$ conformal representations are the conserved currents for the $U(1)$ fermion flavour symmetry and the fermionic stress tensor respectively. These operators are exactly conserved currents so cannot develop anomalous dimensions at any value of $\lambda$. Let us now consider the operator that transforms in the $(4,3)$ representation of the conformal algebra in the free theory. This operator can develop an anomalous dimension only upon combining with an operator in the representation $(5,2)$. However the only spin two single trace operator, the stress tensor, transforms as $(3,2)$ for all values of $\lambda$.

We now make a key assumption that we justify in much more detail below: we assume that, as far as the analysis of leading large $N$ scaling dimensions is concerned, we can simply ignore all mixing of single-trace and multi-trace operators. It follows that, at leading order in $\frac{1}{N}$, the operator in the $(4,3)$ representation cannot develop an anomalous dimension at any value of $\lambda$, simply because at no value of $\lambda$ can there exist a single-trace operator in the $(5,2)$ representation with which the $(4,3)$ operator can combine to form a long representation.

This argument can now be repeated recursively, to demonstrate that the scaling dimensions of all "single trace" spin $s$ operators are exactly protected (at leading order in $N$ ) at all values of $\lambda$. The only way this argument could fail is if the theory underwent a severe phase transition at a finite value of $\lambda$, across which the spectrum of the theory was not continuous.

Note that the non-renormalization theorem relies on the assumption of non mixing of single and multi trace operators (which we will justify in detail below) and the the sparsity of single trace operators in our system. As we will see explicitly below, our non-renormalization theorem will be violated by $\frac{1}{N}$ corrections.

Finally note that nothing in the argument we have presented so far prevents the operator $\bar{\psi} \psi$ from developing an anomalous dimension. However we will proceed to 
argue below that this is also impossible; the scaling dimension of this operator is also protected (as a function of $\lambda$ ) in the interacting theory.

\subsection{Explicit form of the current operators}

In subsection 3.1 .2 above we have determined the explicit form of the primary operators that transform in the $(s+1, s)$ representation of the conformal algebra. In the previous subsection we have argued that the interacting theory has primaries with the same quantum numbers at all values of $\lambda$. In this subsection we will determine the explicit form of these primary operators, in the interacting theory, in terms of the bare fermionic fields $\psi$.

In the interacting theory at finite $\lambda$ let $\hat{J}^{(s)}$ denote the currents obtained from the following procedure: replace all derivatives in the expression for $J^{(s)}$ in the free theory (see subsection 3.1.2) with covariant derivatives. Explicitly, for $s=1 \ldots 4$ we have

$$
\begin{aligned}
\hat{J}_{\mu}^{(1)}= & \bar{\psi} \gamma_{\mu} \psi \\
\hat{J}_{\mu_{1} \mu_{2}}^{(2)}= & \bar{\psi} \gamma_{\mu_{1}}\left(\overrightarrow{D_{\mu_{2}}}-\overleftarrow{D_{\mu_{2}}}\right) \psi \\
\hat{J}_{\mu_{1} \mu_{2} \mu_{3}}^{(3)}= & \frac{1}{6} \bar{\psi} \gamma_{\mu_{1}}\left(3 \overleftarrow{D_{\mu_{2}}} \overleftarrow{D_{\mu_{3}}}-10 \overleftarrow{D_{\mu_{2}}} \overrightarrow{D_{\mu_{3}}}+3 \overrightarrow{D_{\mu_{2}}} \overrightarrow{D_{\mu_{3}}}+2\left(\overleftarrow{D_{\sigma}} \overrightarrow{D^{\digamma}}\right) \eta_{\mu_{2} \mu_{3}}\right) \psi \\
\hat{J}_{\mu_{1} \mu_{2} \mu_{3} \mu_{4}}^{(4)}= & \frac{1}{6} \bar{\psi} \gamma_{\mu_{1}}\left(\overleftarrow{D_{\mu_{2}}} \overleftarrow{D_{\mu_{3}}} \overleftarrow{D_{\mu_{4}}}-7 \overleftarrow{D_{\mu_{2}}} \overleftarrow{D_{\mu_{3}}} \overrightarrow{D_{\mu_{4}}}+7 \overleftarrow{D_{\mu_{2}}} \overrightarrow{D_{\mu_{3}}} \overrightarrow{D_{\mu_{4}}}-\overrightarrow{D_{\mu_{2}}} \overrightarrow{D_{\mu_{3}}} \overrightarrow{D_{\mu_{4}}}\right. \\
& \left.+2 \overleftarrow{D_{\mu_{2}}}\left(\overleftarrow{D_{\sigma}} \overrightarrow{D^{\sigma}}\right) \eta_{\mu_{3} \mu_{4}}-2\left(\overleftarrow{D_{\sigma}} \overrightarrow{D^{\sigma}}\right) \overrightarrow{D_{\mu_{2}}} \eta_{\mu_{3} \mu_{4}}\right) \psi
\end{aligned}
$$

where all indices on the RHS are understood to be symmetrized.

The currents $\hat{J}^{(s)}$ are not automatically tracelessness in their vector indices. The reason for this lack of tracelessness is that covariant derivatives, unlike their ordinary counterparts, do not commute. However the commutator of two covariant derivatives is a field strength. Now according to the classical Chern-Simons equation of motion

$$
\left(F_{\mu \nu}\right)_{j}^{i}=\frac{\pi}{k} \epsilon_{\mu \nu \rho} \bar{\psi}^{i} \gamma^{\rho} \psi_{j}
$$

where the $i$ and $j$ indices are colour indices. Now consider a factor of the field strength $F$ inserted inside a "single trace" fermion bilinear. By the equation of motion cited above ${ }^{15}$, this insertion splits the "single trace" into a "double trace" operator divided by $k$. Further factors of $F$ inside any of the new resultant "traces" repeats this operation. It follows that the spin $s-2, s-4$ etc components of the current $\hat{J}^{(s)}$ is given (via

\footnotetext{
${ }^{15}$ While the equation 3.15 was derived classically, we believe it also applies quantum mechanically, in an appropriate regulator scheme, as the current $J^{(1)}$ is the unique dimension two, spin one operator in the theory.
} 
the equations of motion) by 'multi trace' operators that are schematically take the form $\frac{s^{m}}{k^{m-1}}$ where $s$ represents any "single trace" operator so $s^{m}$ stands for an $m$ trace operator.

The fact that the operators $\hat{J}^{(s)}$ are not traceless means that these currents are not of definite spin; they include components of spin $s$, spin $s-2$ etc. Let us define the interacting currents $J^{(s)}$ as the projection of $\hat{J}^{(s)}$ to its spin $s$ component, i.e. the projection that removes all traces from $\hat{J}^{(s)} . J^{(s)}$ is, by definition, a spin $s$ current, and is of power counting dimension $s+1$. As we have explained above, $J^{(s)}$ and $\hat{J}^{(s)}$ differ only by "multi trace" expressions.

Now the primary operator that transforms in the $(s+1, s)$ representation of the conformal algebra is necessarily an expression of spin $s$ and of power counting dimension $s+1$ (we use a renormalization scheme in which operators can mix only if they have equal classical dimension). The full set of such operators is easily enumerated; in the free theory it consists of those descendents of the primaries $(j+1, j)$ that are of dimension $s+1$ and spin $s$ (clearly this requires $j \leq s$ ). Note that (in the free theory) these are all single trace operators. All spin $s$ multitrace operators in the free theory have dimension greater than or equal to $s+2$.

In the interacting theory, the full set of operators of spin $s$ and classical dimension $s+1$, is given by replacing all derivatives by covariant derivatives in the free answer and then projecting onto the spin $s$ component (i.e. removing all traces). As above, the projection onto spin $s$ leaves the "single trace" part of the operator untouched, but adds "multi trace" operators to the mix.

Now let us compute the divergence of the most general possible spin $s$, classical dimension $s+1$ current listed above in the interacting theory. The computation of this divergence differs from the same computation in the free theory in three ways. First covariant derivatives do not commute, and that results in extra factors of the field strength; as we have explained above such factors modify the multi trace parts of the answer but leave the "single trace" part of the answer untouched. Second, as explained above, the current $J^{(s)}$ has extra multitrace operators as compared to the free current. This additional complication also affects only the multi trace parts of the answer. Finally the fermion equation of motion could be quantum mechanically modified, but any such modification is necessarily in terms of 'multi trace' operators.

In other words the single trace part of the divergence of the general spin $s$ current in the interacting theory is identical to the result of the same computation in the free theory (after replacing derivatives with covariant derivatives). However the interacting divergence includes, in addition, several multi trace contributions that are absent in the free theory. 
Now in subsection 3.1 .2 we computed the unique operator of dimension $s+1$ and spin $s$, in the free theory, that is also conserved. The interacting theory possesses no exactly conserved operator of this dimension. The operator $J^{(s)}$ comes closest to a conserved current, in that it is the unique current that obeys the schematic equation

$$
\partial \cdot J^{(s)} \sim \frac{1}{k} J J+\frac{1}{k^{2}} J J J
$$

(on the RHS of the equation above the symbol $J$ refers either to a current or to a descendent of a current; the important point in this equation is that the RHS contains no single trace pieces). 16 In other words $J^{(s)}$ is the unique spin $s$ and classical dimension $s+1$ field in the interacting theory whose divergence has no single trace component.

As we will see below, the operator $J^{(s)}$ constructed above, may be identified with unique dimension $s+1$ spin $s$ primary of the interacting theory. Before proceeding we will first, however, give an example of how our abstract and exact construction of $J^{(s)}$, (as the projector onto the spin $s$ sector of the current $\tilde{J}^{s}$ ) may actually be practically implemented at leading order in $\lambda$, for the special case of the current $J^{(3)}$. For this purpose in the next subsection we evaluate the current $J^{(3)}$ and its divergence using the classical (but interacting) field equations. In the subsequent subsection we return to the demonstration that the dimension of $J^{(s)}$ is $s+1$.

\subsection{1 $J^{(3)}$ as an example}

The trace of $\hat{J}^{(3)}$ is given by

$$
\hat{J}_{\mu \nu}^{(3) \nu}=\frac{1}{6}\left[\bar{\psi} \gamma_{\mu}\left(\vec{D}^{2}+\overleftarrow{D}^{2}\right) \psi+\bar{\psi} \gamma^{\nu}\left(\left[\vec{D}_{\nu}, \vec{D}_{\mu}\right]+\left[\overleftarrow{D}_{\mu}, \overleftarrow{D}_{\nu}\right]\right) \psi\right]
$$

Using the identities listed in Appendix $\mathrm{E}$

$$
\begin{aligned}
\hat{J}_{\mu \nu}^{(3) \nu} & =-\frac{1}{12} \bar{\psi} F^{\rho \sigma} \epsilon_{\rho \sigma \nu}\left(\gamma^{\nu} \gamma_{\mu}+\gamma_{\mu} \gamma^{\nu}\right) \psi \\
& =-\frac{1}{6} \bar{\psi} F^{\rho \sigma} \epsilon_{\rho \sigma \mu} \psi \\
& =\frac{\pi}{3 k}(\bar{\psi} \psi)\left(\bar{\psi} \gamma^{\mu} \psi\right) .
\end{aligned}
$$

In the last line we used the equation of motion for the field strength $F$ (see Appendix E). Upon projecting out the trace we find

$$
J_{\mu_{1} \mu_{2} \mu_{3}}^{(3)}-\hat{J}_{\mu_{1} \mu_{2} \mu_{3}}^{(3)}=-\frac{\pi}{5 k} \eta_{\left(\mu_{1} \mu_{2}\right.}(\bar{\psi} \psi)\left(\bar{\psi} \gamma_{\left.\mu_{3}\right)} \psi\right)
$$

\footnotetext{
${ }^{16}$ The absence of higher trace operators on the RHS of this equation follows from a consideration of quantum numbers. The is of spin $s-1$ and classical dimension $s+2$. Recall that $\Delta-s \geq 1$ for all single trace operators. It follows that $\Delta-s \geq m$ for $m$ trace operators, and so $m \leq 3$.
} 
As we have explained, the currents $J^{(s)}$ are not expected to be exactly divergence free for $s \geq 3$. On the other hand the divergence of the currents $J^{(1)}$ and $J^{(2)}$ vanishes even in the interacting theory (this is obvious for $J^{(1)}$ and is also true for the stress tensor $T^{\mu \nu}$ as it can be explicitly checked). In Appendix E we have also explicitly computed the divergence of $J^{(3)}$ in the classical but interacting fermion theory. Our result is (see Appendix E.3)

$$
\partial^{\mu} J_{\mu \nu_{1} \nu_{2}}^{(3)}=-\frac{16 \pi}{5 k}\left[\eta_{\nu_{1} \nu_{2}}\left(\partial^{\mu} J^{(0)}\right) J_{\mu}^{(1)}-3\left(\partial_{\left(\nu_{1}\right.} J^{(0)}\right) J_{\left.\nu_{2}\right)}^{(1)}+2 J^{(0)} \partial_{\left(\nu_{1}\right.} J_{\left.\nu_{2}\right)}^{(1)}\right]
$$

We have presented our result in terms of the scalar "current" $J^{(0)}=\bar{\psi} \psi$ and the vector current $J_{\mu}^{(1)}=\bar{\psi} \gamma_{\mu} \psi$.

The equation (3.19) has been derived classically; it could certainly receive quantum corrections. However all such corrections are necessarily of higher order in $\lambda$; at small $\lambda\left(3.19\right.$ gives the leading order contribution to the divergence of $J^{(3)}$.

\subsection{Anomalous dimensions of the current operators}

We will now use the fact that the operators $J^{(s)}$ obey equations of the form 3.16 to argue that the scaling dimension of the operator $J^{(s)}$ is $s+1$ up to corrections of order $\frac{1}{N}$. We will also explain how the knowledge of the precise form of the RHS of 3.16 can immediately be converted into a computation of the anomalous dimensions of $J^{(s)}$ at leading order in $\frac{1}{N}$, and systematically in the $\frac{1}{N}$ expansion.

Let us first review how it follows that a conserved spin $s$ current has $\Delta=s+1$. For this purpose we use the state operator map, and denote the state dual to the operator $O$ by $|O\rangle$. The standard argument takes the schematic form

$$
\langle\partial J \mid \partial J\rangle=\langle J|[K, P]| J\rangle=(\Delta-s-1)\langle J|| J\rangle
$$

(see below for the argument with all indices in place). If $\langle\partial J \mid \partial J\rangle$ vanishes then it follows immediately that $\Delta=s+1$. In the our theory $\langle\partial J \mid \partial J\rangle$ does not quite vanish (see (3.16)). However as we will argue below, (3.16) determines it to be a $\frac{1}{N}$ times a product of known two point functions.

In order to all see this in detail we start with some preliminaries. In radial quantization, $J_{\underline{\mu}}^{(s)}$ inserted at the origin corresponds to a state $\left|J_{\underline{\mu}}^{(s)}\right\rangle$ on the sphere. For convenience we introduce a set of differently normalized currents, $j_{\underline{\mu}}^{(s)}$, whose corresponding states $\left|j_{\underline{\mu}}^{(s)}\right\rangle$ obey

$$
\left\langle j_{\underline{\mu}}^{(s)} \mid j_{\underline{\nu}}^{\left(s^{\prime}\right)}\right\rangle=\delta_{s s^{\prime}} \delta_{\underline{\mu}, \underline{\nu}}
$$

where $\delta_{\mu, \underline{\nu}}$ is 1 if $\underline{\mu}, \underline{\nu}$ are the same set of indices up to permutation and 0 otherwise. $J^{(s)}$ and $j^{(s)}$ are related by $J_{\underline{\mu}}^{(s)}=a_{s} j_{\underline{\mu}}^{(s)}$, where the tree level result for the normalization 
constant $a_{s}$ is computed in Appendix B.2.2. Using the inversion $x_{\mu}^{\prime}=x_{\mu} / x^{2}$, the two point function in position space is related by

$$
\begin{aligned}
\left\langle j_{\underline{\mu}}^{(s)}(x) j_{\underline{\underline{s}}}^{(s)}(0)\right\rangle & =(-)^{s} x^{-2-2 \delta_{s}} \frac{\partial x^{\prime \sigma_{1}}}{\partial x^{\mu_{1}}} \cdots \frac{\partial x^{\prime \sigma_{s}}}{\partial x^{\mu_{s}}}\left\langle j_{\underline{\underline{a}}}^{(s)}\left(x^{\prime}\right) \mid j_{\underline{\underline{p}}}^{(s)}(0)\right\rangle \\
& =(-)^{s} x^{-2 s-2-2 \delta_{s}} \prod_{i=1}^{s}\left(\delta_{\mu_{i}}^{\sigma_{i}}-\frac{2 x^{\sigma_{i}} x^{\mu_{i}}}{x^{2}}\right) \cdot\left\langle j_{\underline{\sigma}}^{(s)} \mid j_{\underline{\nu}}^{(s)}\right\rangle .
\end{aligned}
$$

where $\delta_{s}$ is a possible anomalous dimension for $J^{(s)}$. In the second step we moved $j_{\underline{\sigma}}^{(s)}\left(x^{\prime}\right)$ to $j_{\underline{\sigma}}^{(s)}(0)$ at no cost, since the difference is a conformal descendant, which is orthogonal to $|j(0)\rangle$. One can analogously work out the momentum space two point function.

We can also translate 3.16 into the language of states in radial quantization, schematically of the form

$$
P^{\mu}\left|j_{\mu \mu_{1} \cdots \mu_{s-1}}^{(s)}\right\rangle=\frac{1}{\sqrt{N}} A|j j\rangle+\frac{1}{N} B|j j j\rangle .
$$

Taking its norm (using that $P^{\dagger}=K$ ), we have

$$
\left\langle j_{\mu \mu_{1} \cdots \mu_{s-1}}^{(s)}\left|K^{\mu} P^{\nu}\right| j_{\nu \nu_{1} \cdots \nu_{s-1}}^{(s)}\right\rangle=\frac{1}{N} A^{2}\langle j j \mid j j\rangle+\mathcal{O}\left(\frac{1}{N^{2}}\right)
$$

Since $\left|j_{\nu \nu_{1} \cdots \nu_{s-1}}^{(s)}\right\rangle$ is a conformal primary, it is annihilated by $K_{\mu}$. Using the conformal algebra, the LHS of 3.23 is equal to

$$
\begin{aligned}
& \left\langle j_{\mu \mu_{1} \cdots \mu_{s-1}}^{(s)}\left|\left[K^{\mu}, P^{\nu}\right]\right| j_{\nu \nu_{1} \cdots \nu_{s-1}}^{(s)}\right\rangle=2\left\langle j_{\mu \mu_{1} \cdots \mu_{s-1}}^{(s)}\left|\left(\delta^{\mu \nu} D+M^{\mu \nu}\right)\right| j_{\nu \nu_{1} \cdots \nu_{s-1}}^{(s)}\right\rangle \\
& =2\left[\left(s+1+\delta_{s}\right)\left\langle j_{\mu \mu_{1} \cdots \mu_{s-1}}^{(s)} \mid j_{\mu \nu_{1} \cdots \nu_{s-1}}^{(s)}\right\rangle+\left(\delta_{\mu \nu} \delta_{\nu \rho}-\delta_{\nu \nu} \delta_{\mu \rho}\right)\left\langle j_{\mu \mu_{1} \cdots \mu_{s-1}}^{(s)} \mid j_{\rho \nu_{1} \cdots \nu_{s-1}}^{(s)}\right\rangle\right. \\
& \left.+\sum_{i=1}^{s-1}\left(\delta_{\mu \nu_{i}} \delta_{\nu \rho}-\delta_{\nu \nu_{i}} \delta_{\mu \rho}\right)\left\langle j_{\mu \mu_{1} \cdots \mu_{s-1}}^{(s)} \mid j_{\nu \nu_{1} \cdots \nu_{i-1} \rho \nu_{i+1} \cdots \nu_{s-1}}^{(s)}\right\rangle\right] \\
& =2 \delta_{s}\left\langle j_{\mu \mu_{1} \cdots \mu_{s-1}}^{(s)} \mid j_{\mu \nu_{1} \cdots \nu_{s-1}}^{(s)}\right\rangle \text {. }
\end{aligned}
$$

In the last step we used the fact that $j_{\mu_{1} \cdots \mu_{s}}^{(s)}$ is traceless. The order $1 / N$ contribution to the RHS of (3.23) comes from the disconnected four-point function, i.e. schematically

$$
\langle j j \mid j j\rangle=\langle j \mid j\rangle\langle j \mid j\rangle+\mathcal{O}\left(\frac{1}{N}\right) .
$$

This relates the anomalous dimension $\delta_{s}$ at order $1 / N$ to the product of two two-point functions of $J$ of lower spins in the free theory. Note that this method cannot be used in the $s=0$ case. In Appendix E, an explicit two-loop calculation for the $\mathcal{O}(1 / N)$ anomalous dimension of the scalar operator $\bar{\psi} \psi$ is performed. 
As an explicit example, let us consider the spin 3 current $J_{\mu \nu \rho}^{(3)}$, which obeys the anomalous current conservation relation (3.19). In terms of states in radial quantization, we have the (tree level) relation

$$
\left|\psi_{\nu_{1} \nu_{2}}\right\rangle \equiv P^{\mu}\left|j_{\mu \nu_{1} \nu_{2}}^{(3)}\right\rangle=c\left[\delta_{\nu_{1} \nu_{2}} P^{\mu}\left|j^{(0)}\right\rangle \otimes\left|j_{\mu}^{(1)}\right\rangle-3 P_{\left(\nu_{1}\right.}\left|j^{(0)}\right\rangle \otimes\left|j_{\left.\nu_{2}\right)}^{(1)}\right\rangle+2\left|j^{(0)}\right\rangle \otimes P_{\left(\nu_{1} \mid\right.}\left|j_{\left.\nu_{2}\right)}^{(1)}\right\rangle\right]
$$

where $c=-\frac{16 \pi}{5 k} \frac{a_{0} a_{1}}{a_{3}}$. Taking its norm and using the conformal algebra commutation relations, we find, in particular,

$$
\begin{aligned}
& \left\langle\psi_{\mu \nu} \mid \psi_{\mu \nu}\right\rangle=252|c|^{2} \\
& =2 \delta_{3}\left\langle j_{\mu \nu \rho}^{(3)} \mid j_{\mu \nu \rho}^{(3)}\right\rangle=20 \delta_{3},
\end{aligned}
$$

and so

$$
\delta_{3}=\frac{63}{5}|c|^{2}=\frac{252}{625} \frac{\lambda^{2}}{N}
$$

to the leading nontrivial order, i.e. two-loop order. There are both planar and nonplanar corrections at higher orders in $\lambda$ and in $1 / N$.

A relation of the form 3.23 holds to higher order in $1 / N$ as well; we would however need the connected four-point functions etc., as well as potential $1 / N$ corrections to the relation (3.16), in order to compute the next order contribution to $\delta_{s}$ in $1 / N$.

\subsection{Anomalous current conservation relations within correla- tion functions}

As we have explained above, the primary operators that transform in the $(s+1, s)$ representation (in the large $N$ limit) obey anomalous conservation equations of the form (3.16). The equation obeyed, in particular, by $J^{(3)}$ (in the classical interacting theory) is listed in 3.19 . The nonlinear equations of form 3.19 carry a lot of information, as we will explore in this subsection.

Let us first investigate the implication of anomalous conservation equations on three point functions of spin $J^{(s)}$ operators at leading order in $N .{ }^{17}$. We find the schematic equation

$$
\partial^{\mu}\left\langle J_{\mu \ldots} J J\right\rangle=\frac{1}{k}\langle J J J J\rangle+\frac{1}{k^{2}}\langle J J J J J\rangle
$$

While the leading behaviour of the second term in 3.29 (which comes from factorizing the 5 pt function into the product of a 2 and 3 pt function) is $\mathcal{O}(1)$ (and so subleading in the large $N$ limit) the leading behaviour of the first term on the RHS (this comes from

\footnotetext{
${ }^{17}$ We have seen in the previous section that the operators $J^{(s)}$ are effectively conserved within two point functions at leading order in $N$
} 
factoring the 4 pt function into a product of two point functions) is $\mathcal{O}(N)$ and so of leading order. It follows that our current operators are not, in general, conserved within three point functions even at leading order in the $\mathcal{O}(1 / N)$ expansion. By equating scaling dimensions on the LHS and RHS of $(3.29)$ it follows immediately that the RHS of (3.29) can be nonzero only when $s \geq s_{1}+s_{2}$. In other words the three point function

$$
\left\langle J^{(s)} J^{\left(s_{1}\right)} J^{\left(s_{2}\right)}\right\rangle
$$

can obey anomalous conservation equations (rather than genuine conservation equations) only if $s_{1}, s_{2}$ and $s_{3}$ violate or saturate the triangle inequality. In all the explicit computations we perform below, it will turn out that the RHS of $(3.29)$ is nonzero only when the triangle inequality is explicitly violated (i.e. it vanishes when $s_{1}=s_{2}+s_{3}$ ). It is possible that this is a general exact result that could perhaps be proved by an analysis of allowed structures for 3 point functions, but we will not attempt such an analysis in this paper.

\subsection{Non-renormalization of the scalar operator $J^{(0)}$}

Let us now apply the arguments of the previous subsection to the three point correlator $\left\langle J^{(3)}(x) J^{(1)}(y) J^{(0)}(z)\right\rangle$. According to the arguments of the previous subsection, and (3.19), the divergence of this three point function w.r.t the variable $x$ is schematically proportional (in the large $\mathrm{N}$ limit) to a term proportional $\left\langle\partial J^{(0)} J^{(0)}\right\rangle\left\langle J^{(1)} J^{(1)}\right\rangle$ plus another term proportional to $\left\langle J^{(0)} J^{(0)}\right\rangle\left\langle\partial J^{(1)} J^{(1)}\right\rangle$. The weight under overall scaling of each of these expressions is $5+2 \Delta_{0}$ where $\Delta_{0}$ is the as yet unknown scaling dimension of the current operator $J^{(0)}$. On the other hand the weight under scaling of the divergence of the three pt function is $6+\Delta_{0}$. Equating these weights we find that $\Delta_{0}=2$. We conclude that, just like their spin $s$ counterparts, the scaling dimension of the scalar current $J^{(0)}$ is not renormalized as a function of $\lambda 18$

This non-renormalization result may be argued for more intuitively as follows: the LHS of (3.19) is of dimension 5. The RHS of the same expression is also of dimension

5 if and only $J^{(0)}$ has dimension 2. The slightly technical argument of the previous paragraph simply makes the argument precise.

\footnotetext{
${ }^{18}$ We thank O. Aharony for discussions on this topic.
} 


\section{Three Point Functions}

\subsection{Quantum corrected operators and their 3-point functions}

In the previous section we demonstrated that the dimension $s+1$ spin $s$ operators primary operators $J_{\mu_{1} \ldots \mu_{s}}^{(s)}$ are given by the following construction. First replace all derivatives in (3.5), (3.7) and (3.8) by covariant derivatives. Then project the resultant currents, $\tilde{J}^{s}$, onto the spin $s$ subsector (i.e. we project all lower spins out of the currents). While this procedure is formally exact, a practical implementation of the projection procedure requires use of equations of motion to express $F_{\mu \nu}$ in terms of fermion bilinears. This replacement could receive quantum corrections; moreover it gives rise to quite complicated expressions involving a summation over multi trace contributions.

All complications having to do with the projection operation disappear when we focus on the particular component of $J^{(s)}$ that is obtained by dotting all of its free indices with any constant null vector $\epsilon$. This is because all multitrace contributions to $J^{(s)}$ come multiplied with factors of the metric with free indices and vanish when all these indices are contracted with $\epsilon$ (we use $(\epsilon)^{2}=0$ ). Consequently, in this subsection, we will work exclusively with the operators obtained by contracting all free indices of $J^{(s)}$ with a constant null vector.

In the gauge employed in this paper there is an additional simplification if the null vector in question is chosen to lie in the $x^{-}$direction. In that case the expression for $J^{(s)}$ involves only $D_{-}$derivatives of $\psi$ and $\bar{\psi}$. In the lightcone gauge employed throughout this paper and in much of this subsection, $A_{-}$vanishes, so that $D_{-}=\partial_{-}$. It follows that this particular component of the spin $s$ current is given directly by the expressions (3.5), (3.7) and (3.8) upon setting $\epsilon=\epsilon^{-}$. Explicitly we have

$$
j^{(s)}(p \mid \varepsilon)=\int \frac{d^{3} q}{(2 \pi)^{3}} \bar{\psi}^{i \alpha}(p-q) j_{\alpha \beta}^{(s)}(q ; p \mid \varepsilon) \psi_{i}^{\beta}(q)
$$

where

$$
\begin{aligned}
& j_{\alpha \beta}^{(0)}(q ; p \mid \varepsilon)=\epsilon_{\alpha \beta}, \\
& j_{\alpha \beta}^{(s)}(q ; p \mid \varepsilon)=\left.\left(\gamma^{+}\right)_{\alpha \beta} e^{\varepsilon^{-}\left(2 q^{+}-p^{+}\right)} \frac{\sin \left(2 \varepsilon^{-} \sqrt{q^{+}\left(p^{+}-q^{+}\right)}\right)}{2 \varepsilon^{-} \sqrt{q^{+}\left(p^{+}-q^{+}\right)}}\right|_{\left(\varepsilon^{-}\right)^{s-1}}, \quad s \geq 1 .
\end{aligned}
$$

(see (3.8)). In this particular case, it is easy to define and compute $\mathcal{J}^{(s)}(p \mid \varepsilon)$, the exact quantum corrected version of the operator $j^{(s)}(p \mid \varepsilon)$. We define

$$
\mathcal{J}^{(s)}(p \mid \varepsilon)=\int \frac{d^{3} q}{(2 \pi)^{3}} \bar{\psi}^{i \alpha}(p-q) \mathcal{J}_{\alpha \beta}^{(s)}(q ; p \mid \varepsilon) \psi_{i}^{\beta}(q)
$$


where $\mathcal{J}_{\alpha \beta}^{(s)}(q ; p \mid \varepsilon)$ is defined to be the sum over all the graphs depicted in Fig. 2. Clearly, $\mathcal{J}_{\alpha \beta}^{(s)}(q ; p \mid \varepsilon)$ obeys the Schwinger-Dyson equation

$\mathcal{J}_{\alpha \beta}^{(s)}(q ; p \mid \varepsilon)=j_{\alpha \beta}^{(s)}(q ; p)-\int \frac{d^{3} \ell}{(2 \pi)^{3}}\left[\gamma^{\mu} \frac{1}{i \ell+\Sigma(\ell)}\right]_{\alpha \gamma} \mathcal{J}_{\gamma \delta}^{(s)}(\ell ; p \mid \varepsilon)\left[\frac{1}{i(\ell-\not p)+\Sigma(\ell-p)} \gamma^{\nu}\right]_{\delta \beta} G_{\mu \nu}(\ell-q)$.

where $j_{\alpha \beta}^{(s)}(q ; p \mid \varepsilon)$ is the classical spin- $s$ vertex.

In the special case of $s=1$, it is not difficult to define, and set up an integral equation (analogous to (4.4) ) for the exact quantum corrected operator for arbitrary polarization vector (spin 1 is special because the expression for the current contains no derivatives).

Once we have solved the integral equation (4.4), three point functions of the corresponding operators are determined simply by Wick contraction using the exact fermion propagator. The exact expression for two point function $\left\langle\mathrm{O}_{1} \mathrm{O}_{2}\right\rangle$ may also be obtained by Wick contracting the exact operator $O_{1}$ with the bare operator $O_{2}$ (or vica versa).

We postpone all discussion of the solutions to the integral equations 4.4 , to future work

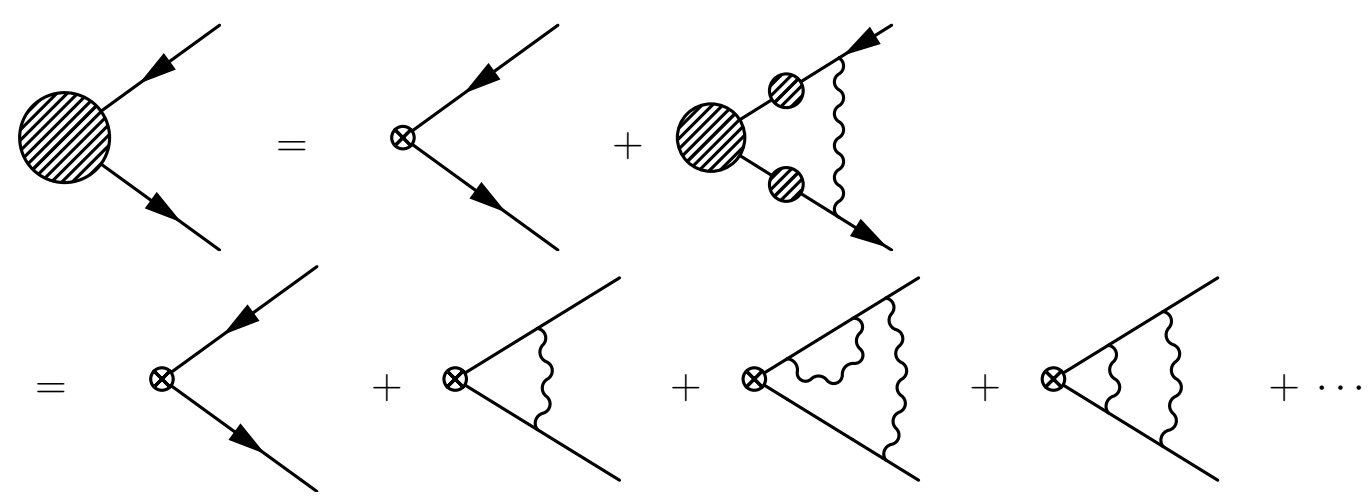

Figure 2: The quantum corrected vertex.

\subsection{Three point functions in perturbation theory}

In the rest of this section, we postpone the attempt to compute correlators exactly in $\lambda$ (at leading order in large $N$ ) but instead turn to study of three point functions of fermion bilinear operators/currents $J^{(s)}$ perturbatively in $\lambda$.

We will begin by writing down the three point functions of currents in the free fermion theory, and describe some general structures of three point functions of higher 
spin currents as constrained by conformal symmetry. While the full tensor structure of the three point functions $\langle J J J\rangle$ of currents of nonzero spin is somewhat complicated, it is constrained by conformal symmetry to be the linear combination of finitely many independent structures. In fact, as shown in [36], for conserved higher spin currents all parity preserving structures of $\left\langle J^{\left(s_{1}\right)} J^{\left(s_{2}\right)} J^{\left(s_{3}\right)}\right\rangle$ can be realized by free scalars and by free fermions, while a parity odd structure of $\left\langle J^{\left(s_{1}\right)} J^{\left(s_{2}\right)} J^{\left(s_{3}\right)}\right\rangle$ exists when the three spins $s_{1}, s_{2}, s_{3}$ obey triangular inequality ${ }^{19}$ The latter cannot be realized with free fields. One of the main results of this section is that the parity odd structure is indeed present in the interacting Chern-Simons-fermion theory.

The parity symmetry of Chern-Simons-fermion theory may be restored by simultaneously taking $k \rightarrow-k$. Therefore, in perturbation theory, the parity invariant tensor structures of $\langle J J J\rangle$ occur at even loop order, while the parity odd tensor structures occur at odd loop order. We will analyze the one-loop contribution to the parity odd structure of $\left\langle J^{(1)} J^{(1)} J^{(2)}\right\rangle$ and $\left\langle J^{(2)} J^{(2)} J^{(2)}\right\rangle$, and the two-loop contribution to the parity even structure of $\left\langle J^{\left(s_{1}\right)} J^{\left(s_{2}\right)} J^{\left(s_{3}\right)}\right\rangle$ for general nonzero $s_{1}, s_{2}, s_{3}$. The results will be summarized in section 4.3, while the details of the computation are left to Appendix $\mathrm{G}$. These results will be very useful in comparing with potentially holographically dual higher spin gauge theories in $A d S_{4}$ (as will be discussed in section 5).

\subsection{Three point functions in the free fermion theory}

As seen before, a generating function for the conserved higher spin currents in the free fermion theory is

$$
J(x, \varepsilon)=\bar{\psi}(x) \gamma \cdot \varepsilon f(\varepsilon \cdot \overleftarrow{\partial}, \varepsilon \cdot \vec{\partial}) \psi(x)
$$

where

$$
f(u, v)=e^{u-v} \frac{\sin (2 \sqrt{u v})}{2 \sqrt{u v}}
$$

and $\varepsilon$ is a null vector. By expanding to $\varepsilon^{s}$ terms, this gives the spin-s currents for all nonzero $s$. The scalar operator $\bar{\psi} \psi$ need to be considered separately. It is useful to write the null polarization vectors in a bispinor form, $\varepsilon_{\alpha \beta}=\lambda_{\alpha} \lambda_{\beta}$. Since $\partial^{2}$ and $\not \partial$ annihilate $\psi$ by the free equation of motion, we can treat $\partial_{\mu}$ as a null vector as well when acting on $\psi$, and make the substitutions

$$
\overleftarrow{\partial}_{\alpha \beta} \rightarrow \xi_{\alpha} \xi_{\beta}, \quad \vec{\partial}_{\alpha \beta} \rightarrow \eta_{\alpha} \eta_{\beta}
$$

where $\xi_{\alpha}, \eta_{\alpha}$ are formal spinorial symbols. We can write the generating current as

$$
J(x, \lambda)=(\bar{\psi} \lambda) \frac{e^{(\lambda(\xi+i \eta))^{2}}-e^{(\lambda(\xi-i \eta))^{2}}}{4(\lambda \xi)(\lambda \eta)}(\lambda \psi) .
$$

\footnotetext{
${ }^{19}$ Recall that when the spins $s_{1}, s_{2}, s_{3}$ obey the triangular inequality, the non-conservation of the currents in our theory does not show up in 3-point functions at leading order at large $N$.
} 
The equation of motion can be implemented by $\eta \psi=0$ and $\bar{\psi} \xi=0$. We may then make a further replacement

$$
\psi_{\alpha} \rightarrow \eta_{\alpha} \phi, \quad \bar{\psi}_{\alpha} \rightarrow \bar{\phi} \xi_{\alpha}
$$

where $\phi$ is a formal object which behaves as a free massless scalar field, while keeping in mind that an extra minus sign comes with a fermion loop. We can then write

$$
J(x, \lambda)=\frac{1}{4} \bar{\phi}\left[e^{(\lambda(\xi+i \eta))^{2}}-e^{(\lambda(\xi-i \eta))^{2}}\right] \phi .
$$

As a simple check, the divergence of the current is obtained from

$$
\left(\partial_{\lambda} \not \partial \partial_{\lambda}\right) J(x, \lambda) \rightarrow\left[\left(\xi \partial_{\lambda}\right)^{2}+\left(\eta \partial_{\lambda}\right)^{2}\right] J(x, \lambda)=\left((\xi+i \eta) \partial_{\lambda}\right)\left((\xi-i \eta) \partial_{\lambda}\right) J(x, \lambda)
$$

which obviously vanishes.

Now let us compute the three point function $\langle J J J\rangle$ for free fermions. The fermion propagator is

$$
\left\langle\psi_{\alpha}(x) \bar{\psi}_{\beta}\left(x^{\prime}\right)\right\rangle=\frac{1}{4 \pi} \frac{\left(\not x-\not x^{\prime}\right)_{\alpha \beta}}{\left|x-x^{\prime}\right|^{3}}=-\not_{\alpha \beta} \frac{1}{4 \pi\left|x-x^{\prime}\right|} \rightarrow i \eta_{\alpha} \xi_{\beta}^{\prime}\left\langle\phi(x) \bar{\phi}\left(x^{\prime}\right)\right\rangle
$$

Here we are making the identification $\xi_{\beta}^{\prime} \sim i \eta_{\beta}$ when acting on $\left\langle\phi(x) \bar{\phi}\left(x^{\prime}\right)\right\rangle$, so as to be consistent with $\overleftarrow{\partial}_{\alpha \beta}^{\prime}=\xi_{\alpha}^{\prime} \xi_{\beta}^{\prime} \sim-\eta_{\alpha} \eta_{\beta}=-\vec{\partial}_{\alpha \beta}$. The three point functions for the generating current, $\left\langle J\left(x_{1}, \lambda_{1}\right) J\left(x_{2}, \lambda_{2}\right) J\left(x_{3}, \lambda_{3}\right)\right\rangle$, can be then written as

$$
\begin{aligned}
& \frac{1}{(4 \pi)^{3}} \prod_{i=1}^{3}\left[e^{\left(\lambda_{i}\left(\xi_{i}+i \eta_{i}\right)\right)^{2}}-e^{\left(\lambda_{i}\left(\xi_{i}-i \eta_{i}\right)\right)^{2}}\right] \frac{1}{\left|x_{i, i+1}\right|}+(1 \leftrightarrow 2) \\
& =\frac{1}{(4 \pi)^{3}} \sum_{r_{i}= \pm 1} \prod_{i=1}^{3} r_{i} \exp \left[\left(\sqrt{-\varepsilon_{i} \cdot \overleftarrow{\partial}_{i}}+r_{i} \sqrt{\varepsilon_{i} \cdot \vec{\partial}_{i}}\right)^{2}\right] \frac{1}{\left|x_{i, i+1}\right|}+(1 \leftrightarrow 2),
\end{aligned}
$$

where $\overleftarrow{\partial}_{i}$ and $\vec{\partial}_{i}$ act cyclically along the product. We can further rescale $\varepsilon_{i} \rightarrow t_{i} \varepsilon_{i}$ expand this generating function in $t_{i}$, and obtain up to normalization the three point functions of three currents of nonzero spins

$$
\sum_{s_{1}, s_{2}, s_{3}} t_{1}^{s_{1}} t_{2}^{s_{2}} t_{3}^{s_{3}}\left\langle J_{s_{1}}\left(x_{1}, \varepsilon_{1}\right) J_{s_{2}}\left(x_{2}, \varepsilon_{2}\right) J_{s_{3}}\left(x_{3}, \varepsilon_{3}\right)\right\rangle .
$$

It follows from essentially the same proof as in the appendix of [32] that an alternative generating function can be written in a simple closed form

$$
\begin{aligned}
& \sum_{s_{1}, s_{2}, s_{3}} \frac{2^{s_{1}+s_{2}+s_{3}} s_{1} ! s_{2} ! s_{3} !}{\left(2 s_{1}\right) !\left(2 s_{2}\right) !\left(2 s_{3}\right) !} t_{1}^{s_{1}} t_{2}^{s_{2}} t_{3}^{s_{3}}\left\langle J_{s_{1}}\left(x_{1}, \varepsilon_{1}\right) J_{s_{2}}\left(x_{2}, \varepsilon_{2}\right) J_{s_{3}}\left(x_{3}, \varepsilon_{3}\right)\right\rangle \\
& =\frac{2}{(4 \pi)^{3}} \frac{1}{\left|x_{12}\right|\left|x_{23}\right|\left|x_{31}\right|} \sinh \frac{Q_{1}+Q_{2}+Q_{3}}{2} \sinh P_{1} \sinh P_{2} \sinh P_{3} .
\end{aligned}
$$

The latter is naturally reproduced from the tree level three point function in the bulk dual type B Vasiliev theory, as shown in 32 . 


\subsection{A few comments on the general structure of three point functions}

Let us begin with an example: the three point function of the stress energy tensor $T_{\mu \nu}$, a quantity of interest in all CFTs. There are three linearly independent tensor structures for $\left\langle T_{\mu_{1} \nu_{1}}\left(x_{1}\right) T_{\mu_{2} \nu_{2}}\left(x_{2}\right) T_{\mu_{3} \nu_{3}}\left(x_{3}\right)\right\rangle$ that are allowed by conformal symmetry (together with current conservation) [34], 35], [36]. In other words,

$$
\langle T T T\rangle=c_{B}\langle T T T\rangle_{B}+c_{F}\langle T T T\rangle_{F}+c_{o d d}\langle T T T\rangle_{o d d}
$$

Here $\langle T T T\rangle_{B}$ stands for the three point function of the stress energy tensor of a free massless scalar, $\langle T T T\rangle_{F}$ that of a free massless fermion, both of which are parity preserving; $\langle T T T\rangle_{\text {odd }}$ is a parity odd structure that is present only in parity violating interacting CFTs.

When the fermions are coupled to Chern-Simons gauge fields, both $\langle T T T\rangle_{B}$ and $\langle T T T\rangle_{\text {odd }}$ show up in the three point function of $T_{\mu \nu}$. As we will see later, $\langle T T T\rangle_{\text {odd }}$ occurs already at one-loop order in perturbation theory, while $\langle T T T\rangle_{B}$ occurs at twoloop order (together with the free fermion structure $\langle T T T\rangle_{F}$ ). The relevant Feynman integrals are difficult to perform directly for generic configuration of operator insertions and polarizations. Instead, we make use of the conformal symmetry, and examine special limits in the configuration space where only a single tensor structure of interest survives; then all we need to do is to compute the overall coefficient of the surviving tensor structure, which is manageable.

We will make use of both conformal symmetry and current conservation to constrain the three point function $\left\langle J_{s_{1}}\left(x_{1}, \varepsilon_{1}\right) J_{s_{2}}\left(x_{2}, \varepsilon_{2}\right) J_{s_{3}}\left(x_{3}, \varepsilon_{3}\right)\right\rangle$. At leading order in $1 / N$, this assumption is valid, up to the anomalous current conservation relation, of the form $\partial \cdot J \sim \frac{1}{k} J J+\cdots$, discussed in section 2 . The relevant part of the divergence of the higher spin currents is expressed in terms of double trace operators, namely product of two currents, of lower spins. This leads to violating of current conservation in the three point function, but only is one of the three spins, $\left(s_{1}, s_{2}, s_{3}\right)$, is greater than the sum of the other two, in other words $\left(s_{1}, s_{2}, s_{3}\right)$ violating the triangular inequality. Also note that this divergence of the three point function is necessarily parity odd (due to the $1 / k$ factor in the anomalous current conservation relation). So for the parity even structures, and for parity odd structures with $\left(s_{1}, s_{2}, s_{3}\right)$ obeying triangular inequality, we may proceed by assuming current conservation. It is shown in [36] under this assumption that $\left\langle J_{s_{1}}\left(x_{1}, \varepsilon_{1}\right) J_{s_{2}}\left(x_{2}, \varepsilon_{2}\right) J_{s_{3}}\left(x_{3}, \varepsilon_{3}\right)\right\rangle$ must be of the form $\left|x_{12}\right|^{-1}\left|x_{23}\right|^{-1}\left|x_{31}\right|^{-1}$ times a polynomial of appropriate degrees in the conformally in- 
variant objects $P_{i}, Q_{i}, S_{i}$. The latter are defined as

$$
\begin{aligned}
& P_{1}=\frac{\lambda_{2} \not_{23} \lambda_{3}}{x_{23}^{2}}, \quad P_{2}=\frac{\lambda_{3} \not_{31} \lambda_{1}}{x_{31}^{2}}, \quad P_{3}=\frac{\lambda_{1} \not_{12} \lambda_{2}}{x_{12}^{2}}, \\
& Q_{1}=-\lambda_{1}\left(\frac{x_{12}}{x_{12}^{2}}+\frac{\not x_{31}}{x_{31}^{2}}\right) \lambda_{1}, \quad Q_{2}=-\lambda_{2}\left(\frac{\not_{23}}{x_{23}^{2}}+\frac{\not x_{12}}{x_{12}^{2}}\right) \lambda_{2}, \quad Q_{3}=-\lambda_{3}\left(\frac{\not_{31}}{x_{31}^{2}}+\frac{\not x_{23}}{x_{23}^{2}}\right) \lambda_{3}, \\
& S_{1}=\frac{i}{4} \frac{\left|x_{12}\right|}{\left|x_{23}\right|\left|x_{31}\right|}\left[\frac{\left(\lambda_{2} \not_{12} \not_{23} \lambda_{2}\right)\left(\lambda_{3} \not_{23} \lambda_{3}\right)}{x_{12}^{2} x_{23}^{2}}+\left(\lambda_{2} \lambda_{3}\right) \lambda_{2}\left(\frac{x_{12}}{x_{12}^{2}}+\frac{\not x_{23}}{x_{23}^{2}}\right) \lambda_{3}\right] \text {, } \\
& S_{2}=\frac{i}{4} \frac{\left|x_{23}\right|}{\left|x_{31}\right|\left|x_{12}\right|}\left[\frac{\left(\lambda_{3} \not_{23} \not_{31} \lambda_{3}\right)\left(\lambda_{1} x_{31} \lambda_{1}\right)}{x_{23}^{2} x_{31}^{2}}+\left(\lambda_{3} \lambda_{1}\right) \lambda_{3}\left(\frac{x_{23}}{x_{23}^{2}}+\frac{x_{31}}{x_{31}^{2}}\right) \lambda_{1}\right] \text {, } \\
& S_{3}=\frac{i}{4} \frac{\left|x_{31}\right|}{\left|x_{12}\right|\left|x_{23}\right|}\left[\frac{\left(\lambda_{1} x_{31} x_{12} \lambda_{1}\right)\left(\lambda_{2} x_{12} \lambda_{2}\right)}{x_{31}^{2} x_{12}^{2}}+\left(\lambda_{1} \lambda_{2}\right) \lambda_{1}\left(\frac{x_{31}}{x_{31}^{2}}+\frac{x_{12}}{x_{12}^{2}}\right) \lambda_{2}\right] \text {. }
\end{aligned}
$$

where again we wrote the null polarization vector $\varepsilon_{i}$ in the bispinor form, in terms of a spinor $\lambda_{i}$. The polynomial in $P, Q, S$ is such that it is homogeneous of degree $2 s_{i}$ in $\lambda_{i}$. Because all quadratic monomials in $S_{i}$ 's can be expressed in terms of $P_{i}, Q_{i}$, we can assume the polynomial to be linear in the $S_{i}$ 's.

Note that under the exchange $\left(x_{1}, \lambda_{1}\right) \leftrightarrow\left(x_{2}, \lambda_{2}\right)$,

$$
\begin{aligned}
& P_{1} \rightarrow-P_{2}, \quad P_{2} \rightarrow-P_{1}, \quad P_{3} \rightarrow-P_{3}, \\
& Q_{1} \rightarrow-Q_{2}, \quad Q_{2} \rightarrow-Q_{1}, \quad Q_{3} \rightarrow-Q_{3} .
\end{aligned}
$$

In case of correlators involving currents of identical spins, the symmetry under exchange of those current should be imposed correspondingly.

The $L$-loop contribution should transform under parity with the sign $(-)^{L}$, namely

$$
\left\langle J_{s_{1}}\left(-x_{1},-\varepsilon_{1}\right) J_{s_{2}}\left(-x_{2},-\varepsilon_{2}\right) J_{s_{3}}\left(-x_{3},-\varepsilon_{3}\right)\right\rangle_{L-\text { loop }}=(-)^{L}\left\langle J_{s_{1}}\left(x_{1}, \varepsilon_{1}\right) J_{s_{2}}\left(x_{2}, \varepsilon_{2}\right) J_{s_{3}}\left(x_{3}, \varepsilon_{3}\right)\right\rangle_{L-\text { loop }}
$$

for $s_{1}, s_{2}, s_{3}>0$. The spin 0 case is special because $J_{0}$ is parity odd unlike $J_{s}$ for $s>0$. We have therefore

$$
\begin{aligned}
& \left\langle J_{s_{1}}\left(-x_{1},-\varepsilon_{1}\right) J_{s_{2}}\left(-x_{2},-\varepsilon_{2}\right) J_{0}\left(-x_{3}\right)\right\rangle_{L}=(-)^{L+1}\left\langle J_{s_{1}}\left(x_{1}, \varepsilon_{1}\right) J_{s_{2}}\left(x_{2}, \varepsilon_{2}\right) J_{0}\left(x_{3}\right)\right\rangle_{L}, \\
& \left\langle J_{s_{1}}\left(-x_{1},-\varepsilon_{1}\right) J_{0}\left(-x_{2}\right) J_{0}\left(-x_{3}\right)\right\rangle_{L}=(-)^{L}\left\langle J_{s_{1}}\left(x_{1}, \varepsilon_{1}\right) J_{0}\left(x_{2}\right) J_{0}\left(x_{3}\right)\right\rangle_{L}, \\
& \left\langle J_{0}\left(-x_{1}\right) J_{0}\left(-x_{2}\right) J_{0}\left(-x_{3}\right)\right\rangle_{L}=(-)^{L+1}\left\langle J_{0}\left(x_{1}\right) J_{0}\left(x_{2}\right) J_{0}\left(x_{3}\right)\right\rangle_{L} .
\end{aligned}
$$

For example, $\left\langle J_{0} J_{0} J_{0}\right\rangle$ can only receive contribution from odd loops. Under $x \rightarrow$ $-x, \varepsilon \rightarrow-\varepsilon, P_{i}, Q_{i}$ are invariant while $S_{i j}$ changes sign. So we expect the $L$-loop 
contribution to the three point function of currents of nonzero spins to be of the form

$$
\begin{aligned}
\left\langle J_{s_{1}}\left(x_{1}, \varepsilon_{1}\right) J_{s_{2}}\left(x_{2}, \varepsilon_{2}\right) J_{s_{3}}\left(x_{3}, \varepsilon_{3}\right)\right\rangle_{L} & =\frac{1}{\left|x_{12}\right|\left|x_{23}\right|\left|x_{31}\right|} F\left(s_{1}, s_{2}, s_{3} \mid P, Q\right), \quad L \text { even, } \\
\left\langle J_{s_{1}}\left(x_{1}, \varepsilon_{1}\right) J_{s_{2}}\left(x_{2}, \varepsilon_{2}\right) J_{s_{3}}\left(x_{3}, \varepsilon_{3}\right)\right\rangle_{L} & =\frac{1}{\left|x_{12}\right|\left|x_{23}\right|\left|x_{31}\right|} \sum_{i} S_{i, i+1} G_{i+2}\left(s_{1}, s_{2}, s_{3} \mid P, Q\right), \quad L \text { odd. }
\end{aligned}
$$

Let us now examine the structure of the three point function of the spin 2 current, i.e. the stress energy tensor $T_{\mu \nu}$, in some detail. $F(2,2,2 \mid P, Q)$ contain two possible structures which are that of the free scalar and free fermion theories. At odd loop level, the contribution to $\langle T T T\rangle$, if nonzero, must be proportional to

$$
\frac{S_{1}\left(P_{1}^{2} Q_{1}^{2}+5 P_{2}^{2} P_{3}^{2}\right)+S_{2}\left(P_{2}^{2} Q_{2}^{2}+5 P_{3}^{2} P_{1}^{2}\right)+S_{3}\left(P_{3}^{2} Q_{3}^{2}+5 P_{1}^{2} P_{2}^{2}\right)}{\left|x_{12}\right|\left|x_{23}\right|\left|x_{31}\right|}
$$

We would like to see whether this structure appears at one loop (at planar level). If such a term is present at one-loop, it would be the entire one-loop contribution to $\langle T T T\rangle$ since it is the only tensor structure allowed by conformal symmetry. Therefore, it suffices to determine the overall coefficient by taking some limit in which the tensor structure simplifies but stays nonzero.

Similarly, there is a parity violating structure for $\langle j j T\rangle$, where $j$ is the spin 1 flavor current. It is proportional to

$$
\frac{2 S_{1} P_{2}^{2}+2 S_{2} P_{1}^{2}+S_{3} Q_{3}^{2}}{\left|x_{12}\right|\left|x_{23}\right|\left|x_{31}\right|} .
$$

Let us consider the special configuration in which all three polarization vectors (or spinors) are equal, namely $\lambda_{i}=\lambda(i=1,2,3)$, so that

$$
Q_{1}=-P_{2}-P_{3}
$$

and

$$
S_{1}=\frac{i}{4} \frac{P_{1} \Delta}{\left|x_{12}\right|\left|x_{23}\right|\left|x_{31}\right|}
$$

etc., where we defined

$$
\Delta \equiv \lambda x_{31} x_{12} \lambda=\lambda x_{12} x_{23} \lambda=\lambda x_{23} x_{31} \lambda .
$$

We can write 4.23 as

$$
\frac{i}{4} \Delta \frac{P_{3}\left(P_{1}+P_{2}\right)^{2}+2 P_{1} P_{2}\left(P_{1}+P_{2}\right)}{x_{12}^{2} x_{23}^{2} x_{31}^{2}}
$$


Now consider the limit in which $x_{1}$ and $x_{2}$ are very close, while $x_{3}$ is kept a finite distance away. For instance, $x_{12}=\delta, x_{31}=x, x_{32}=x+\delta$, and $x$ is kept finite. We have now

$$
P_{1}=-\frac{\varepsilon \cdot(x+\delta)}{(x+\delta)^{2}}, \quad P_{2}=\frac{\varepsilon \cdot x}{x^{2}}, \quad P_{3}=\frac{\varepsilon \cdot \delta}{\delta^{2}}, \quad \Delta=\lambda \not \phi \phi \lambda
$$

To simplify things further, we may choose $x \cdot \varepsilon=0$, say by taking $x=t \hat{e}_{0}$ along Euclidean time direction ( $\hat{e}_{0}$ is the unit vector in Euclidean time direction) and $\delta, \varepsilon$ purely spatial. We can choose $\varepsilon$ to lie along light cone direction $x^{-}$. In this case, 4.28) becomes

$$
P_{1}=-\frac{\delta^{+}}{t^{2}+\delta^{2}}, \quad P_{2}=0, \quad P_{3}=\frac{\delta^{+}}{\delta^{2}}, \quad \Delta=\epsilon_{i j k} x^{i} \delta^{j} \varepsilon^{k}=t \delta^{+},
$$

The parity odd tensor structure for $\left\langle j_{\varepsilon}(0) j_{\varepsilon}(-\delta) T_{\varepsilon}(x)\right\rangle$ then reduces to

$$
\frac{i}{4} \frac{t\left(\delta^{+}\right)^{6}}{\delta^{4} t^{2}\left(t^{2}+\delta^{2}\right)^{3}} \rightarrow \frac{i}{4} \frac{\left(\delta^{+}\right)^{4}}{\delta^{4} t^{7}}
$$

where we took the $\delta \rightarrow 0$ limit on the RHS.

Similarly, we can write the parity odd structure of $\langle T T T\rangle$ in this special case $\lambda_{i}=\lambda$ $(i=1,2,3)$ as

$$
\begin{aligned}
& \frac{i}{4} \Delta \frac{P_{3}\left(P_{3}^{2}\left(P_{1}+P_{2}\right)^{2}+5 P_{1}^{2} P_{2}^{2}\right)+P_{1}\left(P_{1}^{2}\left(P_{2}+P_{3}\right)^{2}+5 P_{2}^{2} P_{3}^{2}\right)+P_{2}\left(P_{2}^{2}\left(P_{3}+P_{1}\right)^{2}+5 P_{3}^{2} P_{1}^{2}\right)}{x_{12}^{2} x_{23}^{2} x_{31}^{2}} \\
& =\frac{i}{4} \frac{\Delta}{x_{12}^{2} x_{23}^{2} x_{31}^{2}}\left(P_{1}+P_{2}+P_{3}\right)\left(P_{1} P_{2}+P_{2} P_{3}+P_{3} P_{1}\right)^{2}
\end{aligned}
$$

In the limit of 4.29 , this becomes

$$
\frac{i}{4} \frac{t\left(\delta^{+}\right)^{6}}{\delta^{8}\left(t^{2}+\delta^{2}\right)^{4}} \rightarrow \frac{i}{4} \frac{\left(\delta^{+}\right)^{6}}{\delta^{8} t^{7}}
$$

where the RHS is the $\delta \rightarrow 0$ limit. Note that if we had taken a generic polarization configuration, in the $\delta \rightarrow 0$ limit we would expect the three point function of $T$ to scale like $\delta^{-3} x^{-6}$. To determine the parity odd structure in our limit, we need to go one order higher in $\delta$ than the naive leading order. In the next subsection, we will compute the coefficient of this term.

\subsection{One-loop and two-loop results}

In this subsection, we summarize the planar one-loop and two-loop perturbative results for the three point functions, leaving details of the computation to Appendix G. 
First we consider the one-loop contribution to the scalar three point function $\left\langle J^{(0)}\left(x_{1}\right) J^{(0)}\left(x_{2}\right) J^{(0)}\left(x_{3}\right)\right\rangle$. Note that $J^{(0)}=\bar{\psi} \psi$ is parity odd, and its three point function can only receive contributions at odd loop order, a priori. We find that it in fact vanishes at one-loop, as shown in Appendix G.1. The computation is performed in both Feynman gauge and lightcone gauge; in Feynman gauge there are nontrivial cancellations between different diagrams, whereas in lightcone gauge each diagram vanishes individually.

In fact, we suspect that $\left\langle J^{(0)}\left(x_{1}\right) J^{(0)}\left(x_{2}\right) J^{(0)}\left(x_{3}\right)\right\rangle$ vanishes identically at planar level. This is suggested by the holographic dual, since $J^{(0)}$ is dual to a bulk scalar of mass -2 in $A d S$ units, and such a scalar should have vanishing cubic coupling, otherwise if one imposes the alternative boundary condition in which the dual scalar operator has dimension 1, the tree level three point function would diverge. This is in particular the case for the general parity violating version of Vasiliev's theory. In field theoretic terms, one may be able to see this by considering the UV fixed point obtained from a double trace deformation by $J^{(0)} J^{(0)}$, and relate $\left\langle J^{(0)} J^{(0)} J^{(0)}\right\rangle$ to the corresponding scalar three point function in the UV fixed point theory.

Next, we analyze the one-loop parity odd structure in two examples, $\langle j j T\rangle$ and $\langle T T T\rangle$, where $j=J^{(1)}$ is the $U(1)$ flavor current. Since there is only a single parity odd tensor structure allowed by conformal symmetry and current conservation, we only need to extract the overall coefficient. The computation is mostly easily done in temporal gauge, and by taking the special limit described in the previous subsection. In both cases, we find the answer to be nonzero. The results, expressed in terms of the conformally invariant objects $P_{i}, Q_{i}, S_{i}$ defined in [36] and in the previous subsection, are

$$
\begin{aligned}
& \left\langle j\left(x_{1}, \varepsilon_{1}\right) j\left(x_{2}, \varepsilon_{2}\right) T\left(x_{3}, \varepsilon_{3}\right)\right\rangle_{1-\text { loop }}=-\frac{2 i \lambda}{\pi^{3}} \cdot \frac{2 S_{1} P_{2}^{2}+2 S_{2} P_{1}^{2}+S_{3} Q_{3}^{2}}{\left|x_{12}\right|\left|x_{23}\right|\left|x_{31}\right|}, \\
& \left\langle T\left(x_{1}, \varepsilon_{1}\right) T\left(x_{2}, \varepsilon_{2}\right) T\left(x_{3}, \varepsilon_{3}\right)\right\rangle_{1-l o o p} \\
& \quad=\frac{18 i \lambda}{\pi^{3}} \cdot \frac{S_{1}\left(P_{1}^{2} Q_{1}^{2}+5 P_{2}^{2} P_{3}^{2}\right)+S_{2}\left(P_{2}^{2} Q_{2}^{2}+5 P_{3}^{2} P_{1}^{2}\right)+S_{3}\left(P_{3}^{2} Q_{3}^{2}+5 P_{1}^{2} P_{2}^{2}\right)}{\left|x_{12}\right|\left|x_{23}\right|\left|x_{31}\right|} .
\end{aligned}
$$

Finally, we compute the two-loop parity even structure for $\left\langle J^{\left(s_{1}\right)} J^{\left(s_{2}\right)} J^{\left(s_{3}\right)}\right\rangle$ with general spins. As remarked earlier, this three point function is conserved to leading order in $1 / N$ if the three spins obey triangular inequality, and when the triangular inequality is not obeyed, the non-conserving structure is necessarily parity odd and does not show up at two-loop. So at two-loop order the result is a linear combination of the free scalar and free fermion answer. The computation in Appendix G.3 focuses on extracting the coefficients of the free scalar structure $\langle J J J\rangle_{B}$, by working in the temporal gauge and taking a special limit in which the free fermion structure $\langle J J J\rangle_{F}$ 
while the free scalar structure survives. Our results may be summarized in the following form:

$$
\begin{aligned}
& \left\langle j^{\left(s_{1}\right)}\left(x_{1}, \varepsilon_{1}\right) j^{\left(s_{2}\right)}\left(x_{2}, \varepsilon_{2}\right) j^{\left(s_{3}\right)}\left(x_{3}, \varepsilon_{3}\right)\right\rangle_{2-l o o p} \\
& =c_{B}\left\langle j^{\left(s_{1}\right)}\left(x_{1}, \varepsilon_{1}\right) j^{\left(s_{2}\right)}\left(x_{2}, \varepsilon_{2}\right) j^{\left(s_{3}\right)}\left(x_{3}, \varepsilon_{3}\right)\right\rangle_{B}+c_{F}^{s_{1} s_{2} s_{3}}\left\langle j^{\left(s_{1}\right)}\left(x_{1}, \varepsilon_{1}\right) j^{\left(s_{2}\right)}\left(x_{2}, \varepsilon_{2}\right) j^{\left(s_{3}\right)}\left(x_{3}, \varepsilon_{3}\right)\right\rangle_{F},
\end{aligned}
$$

where the currents are normalized by their two point functions, with

$$
c_{B}=\frac{\pi^{2}}{4} \frac{\lambda^{2}}{\sqrt{N}} .
$$

A nontrivial feature of this result is that $c_{B}$ is independent of the spins $s_{1}, s_{2}, s_{3}$. As will be discussed in section 5 , this property agrees with what one expects from the tree level three point function in the parity violating version of Vasiliev's higher spin gauge theory in $A d S_{4}$. The agreement also suggests that $c_{F}^{s_{1} s_{2} s_{3}}$ should be independent of the spins as well, though we have not computed this explicitly at two-loop order.

\section{The Formal Solution at Large $N$}

\subsection{Exact effective action for $\psi$ and $\Sigma$}

In the large $N$ limit, the exact quantum effective action for the $\Sigma$ field, obtained after integrating out the fermions, is given by (2.44). The field $\Sigma$ referred to above is an auxiliary field introduced as a computational device. The effective action that we are really interested in, in our problem, is the effective action for $\psi$. This quantum effective action contains all information needed to compute scattering of $\psi$ quanta, and also allows the exact computation of correlators of some operators (see the previous subsection). In this subsection we outline how the exact quantum effective action for the fields $\psi$ and $\Sigma$ may be computed in the large $N$ limit.

All interactions in the action 2.41) are of the form $\bar{\psi} \Sigma \psi$; consequently interactions always involve a $\Sigma$ field. However the propagator of the $\Sigma$ field is $\mathcal{O}\left(\frac{1}{N}\right)$. It follows that the only interactions that survive at leading order in large $N$ are those that compensate for every $\Sigma$ propagator with a fermion loop. With this in mind it is easy to convince oneself that the exact quantum effective action of our theory has the following structure at leading order in large $N$.

- 1. The part of the effective action that involves only $\psi$ and $\bar{\psi}$ (i.e. is independent of $\Sigma$ ) is quadratic in fermion fields. The quadratic form is the inverse of the fermion propagator. 
- 2. The bare $\bar{\psi} \Sigma \psi$ interaction in (2.41) is not renormalized. There are no additional interactions between $\Sigma$ and the fermion fields.

- 3. All $\Sigma$ self interactions are obtained from the determinant in 2.44) (see the previous subsection). The expansion of this determinant in powers of $\Sigma$ yields $\Sigma$ self interactions of the form $\Sigma^{k}$ for all $k \geq 2$. The coefficient of the $\Sigma^{k}$ term is diagrammatically described by a one loop $\psi$ graph with $k$ sigma lines sticking out of it.

The net upshot is that, in order to compute the full quantum effective jointly for the $\psi$ and $\Sigma$ fields, we need only compute the exact fermion propagator (we have already done this) together with the effective action (2.44) o the $\Sigma$ fields.

While the quantum effective action of our system is evaluated rather easily, it is not straightforward to determine the $\Sigma$ propagator by inverting its quadratic action. This process is complicated by the fact that $\Sigma$ is a bilocal field, and relative momentum (the difference between the two momenta dual to the two insertion points of $\Sigma$ ) is not conserved along $\Sigma$ propagators. The quadratic term in the action for $\Sigma$ should be thought of as a "matrix" in relative momentum space, and the inversion of this matrix requires some work. We turn to this problem in the next subsection.

\subsection{Integral equation for the four Fermi vertex}

20

Rather than evaluate the $\Sigma$ propagator through its effective action it is actually more straightforward to set up the Schwinger Dyson equations that govern the off shell four fermion scattering graph. This scattering process proceeds via the exchange of a $\Sigma$ propagator, and so is precisely the $\Sigma$ propagator with two fermions sewn onto each of the legs of this propagator. The four fermion scattering process is, in fact a more useful object to compute than the $\Sigma$ propagator itself. The Schwinger Dyson equation that determines this object is easily derived; we find

$$
\int \frac{d^{3} p d^{3} q d^{3} k}{(2 \pi)^{9}} V_{\alpha \beta \gamma \delta}(p, q ; k) \bar{\psi}^{i \alpha}(-k-p) \psi_{j}{ }^{\beta}(p) \bar{\psi}^{j \gamma}(k-q) \psi_{i}^{\delta}(q) .
$$

20 The axial gauge version of the integral equation described in this section was worked out in collaboration with J. Bhattacharya and S. Bhattacharyya. 

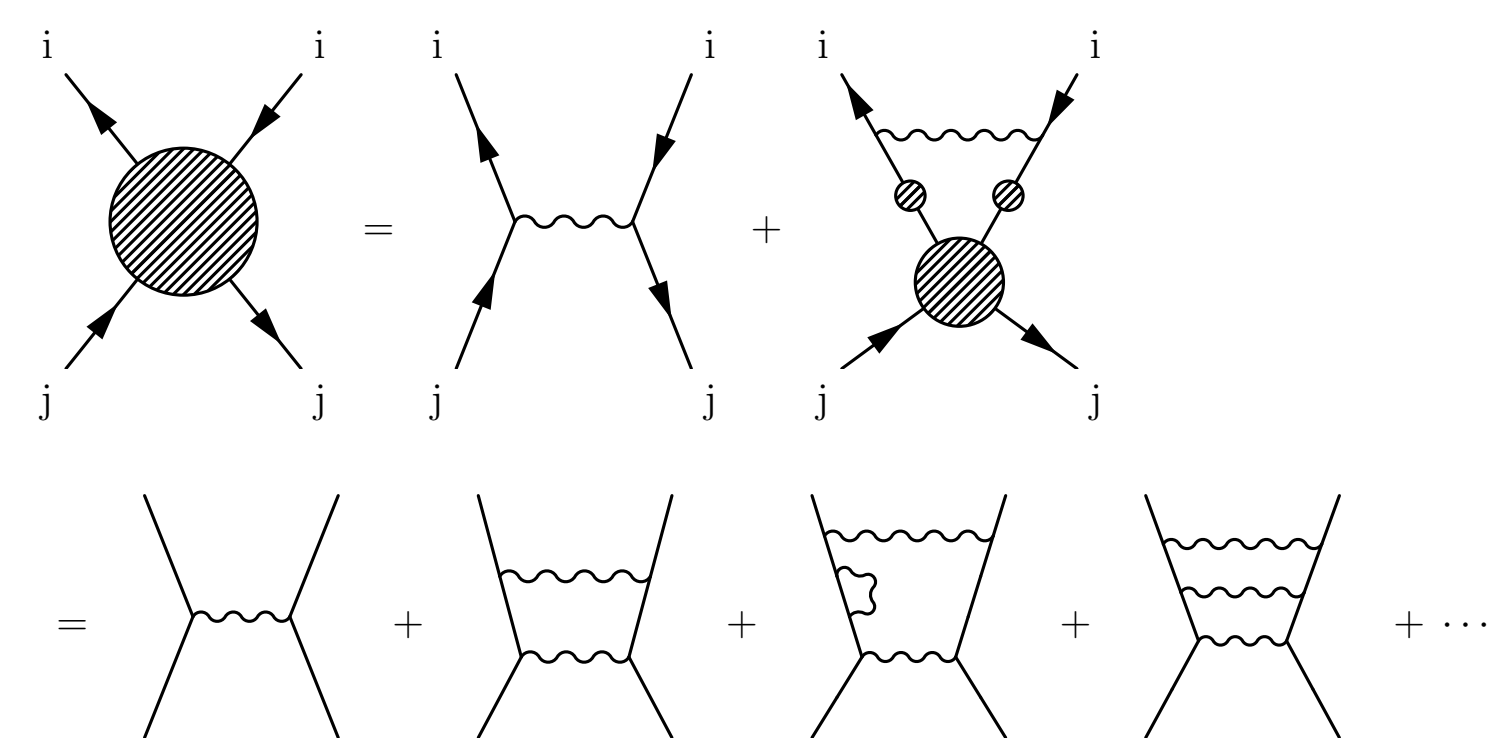

The four-fermion vertex.

$V_{\alpha \beta \gamma \delta}(p, q ; k)$ obeys the Schwinger-Dyson equation

$$
\begin{aligned}
& V_{\alpha \beta \gamma \delta}(p, q ; k)=-\lambda \gamma_{\alpha \beta}^{\mu} \gamma_{\gamma \delta}^{\nu} G_{\nu \mu}(k)-\lambda \int \frac{d^{3} \ell}{(2 \pi)^{3}}\left[\gamma^{\mu} \frac{1}{i(\not p+\not k-\ell)+\Sigma(p+k-\ell)}\right]_{\alpha \sigma} \\
& \quad \times V_{\sigma \beta \gamma \tau}(p, q ; \ell)\left[\gamma^{\nu} \frac{1}{i(\not q-\ell)+\Sigma(q-\ell)}\right]_{\tau \delta} G_{\nu \mu}(\ell) .
\end{aligned}
$$

Substituting in the lightcone gauge gluon propagator, and after a Fierz rearrangement, we obtain the integral equation

$$
\begin{aligned}
& V_{\alpha \beta \gamma \delta}(p, q ; k)=-8 \pi i\left[\delta_{\gamma \beta}\left(\gamma_{-}\right)_{\alpha \delta}-\delta_{\alpha \delta}\left(\gamma_{-}\right)_{\gamma \beta}\right] \frac{k^{-}}{k_{s}^{2}+i \epsilon}-\lambda \int \frac{d^{3} \ell}{(2 \pi)^{3}} V_{\sigma \beta \gamma \tau}(p, q ; \ell) \frac{\ell^{-}}{\ell_{s}^{2}+i \epsilon} \\
& \quad \times\left\{\left[\frac{1}{i(\not p+\not k-\not)+\Sigma(p+k-\ell)}\right]_{\tau \sigma}\left[\gamma_{-} \frac{1}{i(\not l-\ell)+\Sigma(q-\ell)}\right]_{\alpha \delta}\right. \\
& \left.\quad-\left[\gamma_{-} \frac{1}{i(\not p+\not k-\ell)+\Sigma(p+k-\ell)}\right]_{\tau \sigma}\left[\frac{1}{i(\not q-\ell)+\Sigma(q-\ell)}\right]_{\alpha \delta}\right\} .
\end{aligned}
$$

\subsection{Effective action for $\psi$ fields alone}

In this brief subsection we describe an exact way of computing the scattering of an arbitrary number of $\psi$ quanta (or correlators built out of $\psi$ fields) working only with $\psi$ fields alone. The building blocks of our construction are the exact $\psi$ propagator and the exact four Fermi interaction vertex. 
The basic rule is simple. One first draws all the Feynman diagrams that contribute to the process in question using the exact quantum effective action involving both $\Sigma$ field and $\psi$, described above. In computing a $\psi$ scattering process, for instance, we must restrict ourselves to tree graphs with the exact effective action. Graphs for correlators have some loops, involving the fermions that explicitly appear in the operators under study.

The diagrams described in the previous paragraph will in general have some $\Sigma$ internal lines, as well as some $\Sigma 3$ or higher point vertices. We then proceed to blow up every $\Sigma$ interaction vertex into a fermion loop. Once we have done this, the only appearance of $\Sigma$ in graphs is via propagators. Each propagator is sewn, at each end, to two fermion lines. As a consequence each $\Sigma$ internal line carries contributes a factor of the fermion four point function, described in the previous subsection. The exact answer is obtained by enumerating all diagrams that occur in this process, writing down the expressions for these diagrams, and then doing the loop momenta (these loops arise from the blow up of $\Sigma$ interaction vertices, as well as possible loops involving fields inserted in external operators). At every stage, the fermion propagator that participates in this process is the exact propagator.

\subsection{Exact evaluation of correlators}

The effective action of the previous section allows us, quite easily, to compute the correlators singlet operators, each of which is bilinear in the fermion field (see the previous section for an extensive discussion on operators whose correlators we evaluate). In the previous subsection we have already outlined a method to evaluate 3 point functions of the appropriate operators. The methods of this subsection generalize to the evaluation of arbitrary $n$ point functions. We will make no use of the exact operators defined in the previous section, but use the effective action outlined in the previous subsection.

In the rest of this subsection we describe in some detail how we can evaluate the three point functions of fermion bilinear operators using the effective action method (our description generalizes relatively straightforwardly to four and higher point correlators).

In order to perform this computation we only need the fermion effective action expanded to sextic order in the fermions. The exact fermion propagator is determined by (2.2) and (2.3). The 4 Fermi interaction is determined by (5.2) or (5.3). And the six fermion vertex is given by a single fermion loop diagram with three attached fermion bilinears. The fermion propagators to be used in this loop are the exact propagators determined by 2.2)-2.3), while the 4 Fermi vertex factors associated with the attached 
fermion bilinears is the exact Fermi vertex 5.2 .

Given these ingredients, the exact three point function of the three singlet fermionic bilinears is given by the sum of four types of diagrams. The first is a one loop triangle diagram, with the exact fermion propagators, where each vertex in the triangle is one of the external operators.

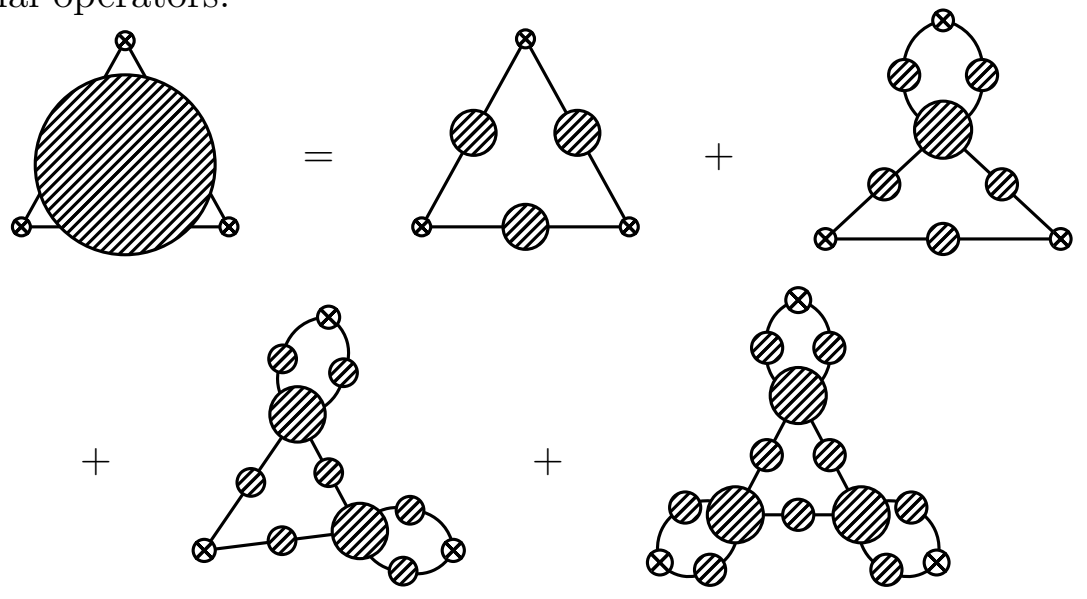

The exact planar three-point function.

The second and third diagrams involve one or two four-fermion vertices. The last diagram involves a single sextic fermion vertex; equivalently, it consists of three fourfermion vertices contracted along a fermion loop using the exact propagator. It follows that, if we could solve both $(2.3)$ and 5.2 we would practically be able to compute all "single trace" three point functions of operators that are quadratic in the fermion fields.

\section{Light-Cone Hamiltonian and $W_{\infty}$ Algebra}

One of the most important features of the Euclidean field theory analysis in section 2.1.3 of this paper is the exact representation of the path integral entirely in terms of the bilocal field $\Sigma$. The use of bilocal fields in field theory and also in the formulation of the large $N$ limit has been discussed by various authors, see e.g. [44], [45], [46], [47]. Also, it naturally appears in discussions of non-commutative field theory [48], [49], $\overline{\mid 50]}$. See also [51], [52], 53] for applications to holography and higher spin theories. These variables, besides highlighting the inherent non-locality of the theory, are natural to discuss the large $N$ limit. The large $N$ saddle point equation is the Schwinger-Dyson equation 2.3 for the propagator. One can then perform a systematic $1 / N$ expansion around the saddle point solution. 
In the following, we will briefly explore the Hamiltonian formulation of the theory in light-cone time and in $A_{+}=0$ gauge. Such a treatment of the path-integral allows one to express the theory in terms of 'equal time' bilocal variables $M(x, y)$ satisfying a $W_{\infty}$ algebra. These operators are also constrained by a quadratic relation $M^{2}=$ $M$, that has appeared before in discussions of non-commutative field theory. The Hamiltonian formulation throws a different light on the structure of this theory, and the $\lambda$ dependence in the exact fermion propagator (2.23) arises in a simple way when evaluated at equal light-cone time.

We will first construct a light-cone Hamiltonian in terms a bilocal field $M(x, y)$ which is defined on a fixed $x^{+}$slice, and then use this Hamiltonian to derive an action for $M(x, y)$ using the method of coadjoint orbits. Here $x^{+}$will serve as a time coordinate and the Hamiltonian that we will construct is conjugate to this variable. Our analysis closely follows [47], applied to the large $N$ limit of $Q C D_{2}$.

We pause to remark that light-cone quantization of any theory has a number of important issues, and (a priori) is not necessarily equivalent to usual time-like quantization. See, e.g., [54]. While we leave careful consideration of these issues for future investigation, we will observe that, applied with appropriate qualification, the lightcone quantization appears to give some simplifying insights into the dynamics of the theory.

We will be working entirely in Minkowski space-time for this section. We use the following explicit representation for gamma-matrices:

$$
\begin{aligned}
& \gamma^{0}=-i \sigma^{2}=\left(\begin{array}{cc}
0 & -1 \\
1 & 0
\end{array}\right), \quad \gamma^{1}=\sigma^{1}=\left(\begin{array}{ll}
0 & 1 \\
1 & 0
\end{array}\right), \quad \gamma^{2}=\sigma^{3}=\left(\begin{array}{cc}
1 & 0 \\
0 & -1
\end{array}\right) \\
& \gamma^{-}=\frac{1}{\sqrt{2}}\left(-\gamma^{0}+\gamma^{1}\right)=\left(\begin{array}{cc}
0 & \sqrt{2} \\
0 & 0
\end{array}\right), \quad \gamma^{-}=\frac{1}{\sqrt{2}}\left(\gamma^{0}+\gamma^{1}\right)=\left(\begin{array}{cc}
0 & 0 \\
\sqrt{2} & 0
\end{array}\right)
\end{aligned}
$$

The fermion is a two-component Dirac spinor which we write as

$$
\psi=\left(\begin{array}{l}
\psi_{-} \\
\psi_{+}
\end{array}\right)
$$

These conventions are similar to the Euclidean conventions with a few minor differences. The lightcone coordinates are a sum of $x^{0}$ and $x^{1}$ and the non-light cone direction is $x^{2}$ (instead of $x_{\text {Eucl. }}^{3}$ ). In Minkowski space, it is natural to define $x^{ \pm}=\left( \pm x^{0}+x^{1}\right) / \sqrt{2}$, which happens to equal $x_{\text {Eucl. }}^{\mp}$, as used elsewhere in the paper.

The action in written in Minkowski space is

$$
S=-\frac{k}{4 \pi} \int \operatorname{tr}\left(A \wedge d A+\frac{2 i}{3} A \wedge A \wedge A\right)-\int d^{3} x\left(i \bar{\psi} \gamma^{\mu} D_{\mu} \psi+i m \bar{\psi} \psi\right)
$$


We obtain the Hamiltonian for the theory in the gauge $A_{+}=0$. As already mentioned $x^{+}$is the 'time' coordinate, and $x^{-}$and $x^{2}$ are the space coordinates. The Gauss constraint arising from the equation of motion of $A_{+}$is:

$$
-\frac{k}{4 \pi}\left(\partial_{-} A_{2}^{c}-\partial_{2} A_{-}^{c}-f^{a b c} A_{-}^{a} A_{2}^{b}\right)+\sqrt{2} \psi_{-}^{\dagger} T^{c} \psi_{-}=0
$$

The momenta conjugate to $\psi_{+}$and $\psi_{+}^{\dagger}$ vanish - this reduction in the number of degrees of freedom is a consequence of the choice of light-cone direction $x^{+}$as the time variable - the corresponding constraints arising from their equations of motion are:

$$
\begin{aligned}
i \sqrt{2} \partial_{-} \psi_{+}+i \partial_{2} \psi_{-}-i m \psi_{-}+\sqrt{2} A_{-}^{a} T^{a} \psi_{+}+A_{2}^{a} T^{a} \psi_{-} & =0 \\
-i \sqrt{2} \partial_{-} \psi_{+}^{\dagger}-i \partial_{2} \psi_{-}^{\dagger}+i m \psi_{-}^{\dagger}+\psi_{+}^{\dagger} \sqrt{2} A_{-}^{a} T^{a}+\psi_{-}^{\dagger} A_{2}^{a} T^{a} & =0
\end{aligned}
$$

The momentum conjugate to $A_{-}^{a}$ is

$$
\pi_{A_{-}}=\frac{\partial \mathcal{L}}{\partial\left(\partial_{+} A_{-}^{a}\right)}=-\frac{k}{4 \pi} A_{2}^{a}
$$

The momentum conjugate to $\psi_{-}$is

$$
\pi_{\psi_{-}^{i}}=\frac{\partial \mathcal{L}}{\partial\left(\partial_{+} \psi_{-}\right)}=i \sqrt{2} \psi_{-}^{\dagger}
$$

The Hamiltonian then takes the following form

$$
\mathcal{H}=i \psi_{-}^{\dagger} \partial_{2} \psi_{+}+i m \psi_{-}^{\dagger} \psi_{-}+\psi_{-}^{\dagger} A_{2}^{a} T^{a} \psi_{+}
$$

Using the residual gauge symmetry to set $A_{-}=0$, we can explicitly solve the constraints to express $A_{2}, \psi_{+}$and $\psi_{+}^{\dagger}$ in terms of $\psi_{-}$and its conjugate momentum $\psi_{-}^{\dagger}$.

The resulting (spatially non-local) Hamiltonian, expressed in momentum space is:

$$
\begin{aligned}
H= & \int \frac{d^{2} p}{4 \pi^{2}}\left(\frac{p_{2}^{2}+m^{2}}{\sqrt{2} p_{+}}\right) \psi_{-}^{\dagger}(-p) \psi_{-}(p) \\
& +\frac{2 \pi}{k} \int \frac{d^{2} p}{4 \pi^{2}} \frac{d^{2} q}{4 \pi^{2}} \frac{d^{2} q^{\prime}}{4 \pi^{2}}\left(\frac{\left(-i p_{2}-m\right)}{p_{-} q_{-}}\right) \psi_{-}^{\dagger}(-p)\left(\psi_{-}^{\dagger}\left(q-q^{\prime}\right) \psi_{-}(p-q)\right) \psi_{-}\left(q^{\prime}\right) \\
& -\frac{2 \pi}{k} \int \frac{d^{2} p}{4 \pi^{2}} \frac{d^{2} q}{4 \pi^{2}} \frac{d^{2} p^{\prime}}{4 \pi^{2}}\left(\frac{i p_{2}-i q_{2}-m}{q_{-}\left(p_{-}-q_{-}\right)}\right) \psi_{-}^{\dagger}(-p)\left(\psi_{-}^{\dagger}\left(q-p^{\prime}\right) \psi_{-}(p-q)\right) \psi_{-}\left(p^{\prime}\right) \\
& +\sqrt{2} \frac{4 \pi^{2}}{k^{2}} \int \frac{d^{2} p}{4 \pi^{2}} \frac{d^{2} q}{4 \pi^{2}} \frac{d^{2} p^{\prime}}{4 \pi^{2}} \frac{d^{2} q^{\prime}}{4 \pi^{2}} \frac{d^{2} q^{\prime \prime}}{4 \pi^{2}} \\
& \left(\frac{1}{p_{-}-q_{-}} \frac{1}{q_{-}^{\prime}} \frac{1}{q_{-}}\right) \psi_{-}^{\dagger}\left(q-p^{\prime}\right)\left(\psi_{-}^{\dagger}(-p) \psi_{-}\left(p^{\prime}\right)\right)\left(\psi_{-}^{\dagger}\left(q^{\prime}-q^{\prime \prime}\right) \psi_{-}\left(p-q-q^{\prime}\right)\right) \psi_{-}\left(q^{\prime \prime}\right)
\end{aligned}
$$


Note that, because we are using light-cone time, and we must choose some prescription to define the inverse of the light-cone momenta when one solves the constraints (6.5) and (6.6) in momentum space. Choosing the principal value prescription of regulating the infra-red divergence effectively eliminates the zero mode of $\psi_{+}$. The only dynamical degree of freedom in the theory is then $\psi_{-}$. As pointed out in [54] in the limit of large $N$, using the principal value prescription in the definition of infra-red divergent quantities like $1 / p_{-}$, in the related theory of two-dimensional $Q C D_{2}$, one can obtain the correct 't Hooft equation that determines the meson spectrum.

\subsection{Bilocal variables satisfying a $W_{\infty}$ algebra}

Let us define the following bilocal operator

$$
M(x, y)=\frac{\sqrt{2}}{N} \psi_{j-}^{\dagger}(x) \psi_{-}^{j}(y)
$$

These operators, which would be the gauge invariant Wilson lines along the $x^{-}$direction in the $A_{-}$gauge if the points $x$ and $y$ were not separated in the 2-direction, constitute the quantum phase space of the light-cone quantized formulation of the theory.

As we will be working in momentum space, it will be more convenient to use

$$
M\left(q, q^{\prime}\right)=\frac{\sqrt{2}}{(2 \pi)^{2} N} \psi_{j-}^{\dagger}(-q) \psi_{-}^{j}\left(q^{\prime}\right)
$$

It is easy to calculate the commutation relations using the basic fermion anticommutation relations:

$$
\left\{\psi_{-j}^{\dagger}(k), \psi_{-}^{i}(k)\right\}=\frac{1}{\sqrt{2}} \delta_{j}^{i}(2 \pi)^{2} \delta^{2}\left(k+k^{\prime}\right) .
$$

These imply that the $M$ 's satisfy the 'quantum' $W_{\infty}$ algebra

$$
\left[M\left(p, p^{\prime}\right), M\left(q, q^{\prime}\right)\right]=\frac{1}{N}\left(M\left(p, q^{\prime}\right) \delta^{2}\left(p^{\prime}-q\right)-M\left(q, p^{\prime}\right) \delta^{2}\left(q^{\prime}-p\right)\right)
$$

Notationally, we may often represent $M$ as an operator acting on a single particle Hilbert space, $\hat{M}$, with

$$
\langle x|\hat{M}| y\rangle=M(x, y)
$$

though $M(x, y)$ is itself an operator acting on the Hilbert space of the quantum field theory, i.e., multi-particle states. Such constructions are familiar from non-commutative field theory. 
In terms of $W_{\infty}$ variables, the Hamiltonian, using the principal value prescription, can be easily expressed as a 'cubic polynomial' in $M$ :

$$
\begin{aligned}
H= & N \int d^{2} p \frac{p_{2}^{2}+m^{2}}{2 p_{-}} M(p, p) \\
& -N \lambda \frac{1}{4 \pi} \int d^{2} p d^{2} q d^{2} q^{\prime} \frac{i p_{2}+m}{p_{-} q_{-}} M\left(p, q^{\prime}\right) M\left(q^{\prime}-q, p-q\right) \\
& -N \lambda \frac{1}{4 \pi} \int d^{2} p d^{2} q d^{2} q^{\prime} \frac{i p_{2}-i q_{2}-m}{q_{-}\left(p_{-}-q_{-}\right)} M\left(p, q^{\prime}\right) M\left(q^{\prime}-q, p-q\right) \\
& -\frac{N \lambda^{2}}{8 \pi^{2} k^{2}} \int d^{2} p d^{2} q d^{2} p^{\prime} d^{2} q^{\prime} d^{2} q^{\prime \prime} \frac{1}{q_{-} q_{-}^{\prime} q_{-}^{\prime \prime}} M\left(p^{\prime}+q^{\prime \prime}, p\right) M\left(q+q^{\prime \prime}, p^{\prime}\right) M\left(p-q^{\prime}, q-q^{\prime}\right)
\end{aligned}
$$

The inverse of $p_{-}$in the above manipulations is assumed to be defined using the principal value prescription.

\subsubsection{Constraint on the bilocal fields}

We now restrict the space of allowed states to be singlets under global gauge transformations i.e. the generators of global $S U(N)$ gauge transformations constrain the wave functions as follows:

$$
\int d x^{-} d x^{2}\left(\psi_{-}^{\dagger b} \psi_{-}^{a}-\frac{1}{N} \delta^{a b} \psi_{-}^{\dagger c} \psi_{-}^{c}\right)|\Psi\rangle=0
$$

We also note that the baryon number operator

$$
B=\frac{\sqrt{2}}{N(2 \pi)^{2}} \int d^{2} p \psi_{-}^{\dagger a}(-p) \psi_{-}^{a}(p)
$$

commutes with the Hamiltonian and hence it is conserved and takes a definite value on physical states. This fact leads to a very important operator constraint in the phase space. From the definition of $M$, it follows that

$$
\begin{aligned}
\hat{M}^{2}\left(p, p^{\prime}\right) & =\int d q M(p, q) M\left(q, p^{\prime}\right)=(1+B) M\left(p, p^{\prime}\right) \\
B & =\int d^{2} p M(p, p)
\end{aligned}
$$

This completes the light-cone Hamiltonian formulation. The (light-cone quantized) phase space operators are the $M$ 's. Their commutation relations satisfy the $W_{\infty}$ Lie algebra. Since the generators of this Lie algebra are represented in terms of the underlying fermions, they are not all independent and satisfy a constraint which is a consequence of gauge invariance and a conserved baryon number. It is clear that we are dealing with a $W_{\infty}$ Hamiltonian system, whose representation is fixed by the constraint $\hat{M}^{2}=(1+B) \hat{M}$. We will restrict ourselves to the $B=0$ sector of this theory. 


\subsection{The path integral and the large- $N$ classical solution}

From the light-cone Hamiltonian one can construct an action (and the path integral) for the theory using standard techniques of geometric quantization viz. the method of coadjoint orbits of the $\left(W_{\infty}\right)$ Lie group. Equivalently the path integral can be derived using a basis of coherent states of the $M$ operators between adjacent time steps. The action one obtains in terms of the 'c-number' bilocal fields is (see [47,49] and references therein),

$$
S_{\text {classical }}=N \frac{i}{4} \int d s d x^{+} \operatorname{tr} \hat{M}\left[\partial_{s} \hat{M}, \partial_{+} \hat{M}\right]-\int d x^{+} H
$$

The first term of the action is the Kirilov-Berry phase. It is written using an auxiliary parameter $s$ besides the time $t$. If $t$ is periodic then $s$ parameterizes a unit disc. We need to impose the boundary condition $M(s=0, t)=0$ and $M(s=1, t)=M(t)$. The classical equations of motion are obtained by varying the action consistent with the $W_{\infty}$ group law and using the above mentioned boundary conditions. These equations are by construction the Hamilton equations of motion: $-i \partial_{+} M=[H, M]$.

The path integral measure over $M$ is constrained by the covariant condition $\hat{M}^{2}=$ $\hat{M}$, which ultimately arises from evaluating the operator constraint in the space of coherent states (see [49]). In particular, to obtain the solution $\langle M\rangle$ that corresponds to the ground state expectation value of $M$, we solve the time-independent equation $[H, M]=0$.

From the commutation relations of the $W_{\infty}$ variables, one can see that any translationally invariant $\langle M\rangle$

$$
\langle M(p, q)\rangle=\delta^{2}\left(p-p^{\prime}\right) f(p)
$$

is a solution provided the constraint $\langle\hat{M}\rangle^{2}=\langle\hat{M}\rangle$ is satisfied. This implies that $f(p)^{2}=$ $f(p)$, which being independent of $\lambda$, is most naturally solved by $f(k)=\theta\left(-\frac{p_{2}^{2}+m^{2}}{p_{-}}\right) \approx=$ $\theta\left(-p_{-}\right){ }^{21}$

It is important to note that this solution is the exact solution in the large- $N$ limit. It allows one to read off the equal-time correlation function for $\psi_{-}$'s, valid to all orders in $\lambda$,

$$
\left\langle\psi_{j-}^{\dagger}\left(q^{\prime}\right) \psi_{-}^{j}(q)\right\rangle=N \frac{1}{\sqrt{2}}(2 \pi)^{2}\left\langle M\left(-q^{\prime}, q\right)\right\rangle
$$

which implies:

$$
\left\langle\psi_{-}^{j}(q) \psi_{j-}^{\dagger}\left(q^{\prime}\right)\right\rangle=N \frac{1}{\sqrt{2}} \theta\left(q_{-}\right)(2 \pi)^{2} \delta^{2}\left(q+q^{\prime}\right)
$$

This is, in fact, one of the components of the equal- "time" fermionic propagator. Using the constraints, we can use this solution to obtain other components of the exact

\footnotetext{
${ }^{21}$ We thank J. David for discussion on this point.
} 
propagator. The fermionic constraints in this large- $N$ solution read:

$$
\begin{aligned}
\psi_{+}(q) & =\frac{i}{\sqrt{2} q_{-}}\left(i q_{2}-\left(m+\lambda I_{*}(-q)\right)\right) \psi_{-}(q) \\
\psi_{+}^{\dagger}\left(q^{\prime}\right) & =\frac{i}{\sqrt{2} q_{-}^{\prime}}\left(i q_{2}^{\prime}-\left(m+\lambda I_{*}\left(q^{\prime}\right)\right)\right) \psi_{-}^{\dagger}\left(q^{\prime}\right)
\end{aligned}
$$

where $I_{*}(-q)=\frac{1}{2 \pi} \int d^{2} p \frac{1}{p_{+}} M(q-p)$.

Let us discuss the regularization of $\left(m+I_{*}(-q)\right)$. First, let us note that if $q_{-} A(q)=$ $J(q)$, then $A(q)=\left(\frac{1}{q_{-}}+h\left(q_{2}\right) \delta\left(q_{-}\right)\right) J(q)$ is a solution for any $h\left(q_{2}\right)$, because $q_{-} \delta\left(q_{-}\right)$ is effectively equal to zero, (assuming $J(q) \neq 1 / q_{-}$at $q_{-}=0$ ). This is ultimately an infrared ambiguity in the inverse of $\partial_{-}$.

Second, note that we will also regulate the vacuum solution $M(q-p)=\lim _{x^{2} \rightarrow 0} \theta(q-$ $p) e^{i k_{2} x^{2}}$ so that $\int d^{2} p M(p, p)=B=0$.

Taking both the infrared ambiguity and the regularization of $M$ into account, we have

$$
\begin{aligned}
I_{*}\left(q_{-}\right) & =\frac{1}{2 \pi} \int d p_{2} d p_{-}\left(\frac{1}{p_{-}}+h\left(p_{2}\right) \delta\left(p_{-}\right)\right) e^{i\left(q_{2}-p_{2}\right) x^{2}} \theta\left(q_{-}-p_{-}\right) \\
& =\frac{1}{2 \pi} \int d p_{2} d p_{-} h\left(p_{2}\right) \delta\left(p_{-}\right) e^{i\left(q_{2}-p_{2}\right) x^{2}} \theta\left(q_{-}-p_{-}\right) \\
& =\frac{1}{2 \pi} \int d p_{2} h\left(p_{2}\right) e^{i\left(q_{2}-p_{2}\right) x^{2}} \theta\left(q_{-}\right) \\
& =\frac{1}{2 \pi} e^{i q_{2} x^{2}} \int d p_{2} h\left(p_{2}\right) e^{-i p_{2} x^{2}} \theta\left(q_{-}\right)
\end{aligned}
$$

Note that although $h\left(p_{2}\right)$ is not specified, it must be dimensionless. Let us take $h\left(p_{2}\right)=$ $h_{0} e^{-\left(p_{2} x^{2}\right)^{2}} \cos \left(p_{2} x^{2}\right)$. Then

$$
\begin{aligned}
I_{*}\left(q_{-}\right) & =\frac{h_{0}}{2 \pi} e^{i q_{2} x^{2}} \int d p_{2} e^{-\left(p_{2} x^{2}\right)^{2}} \cos \left(p_{2} x^{2}\right) e^{-i p_{2} x^{2}} \theta\left(q_{-}\right) \\
& =\frac{h_{0}}{2 \pi} e^{i q_{2} x^{2}} \int d p_{2} e^{-\left(p_{2} x^{2}\right)^{2}} \cos ^{2}\left(p_{2} x^{2}\right) \theta\left(q_{-}\right) \\
& =e^{i q_{2} x^{2}} / x^{2} \theta\left(q_{-}\right) \\
& =\left(1 / x^{2}+i q_{2}\right) \theta\left(q_{-}\right)
\end{aligned}
$$

where, in the last line, we took the limit $x^{2} \rightarrow 0$, and adjusted the numerical constant $h_{0}$.

In the limit $x^{2} \rightarrow 0$ we get a divergent term independent of momentum, which we cancel with the mass counterterm, and a finite term remains, $i q_{2}$; hence $\left(m+I_{*}(-q)\right)=$ $i q_{2}$. Regardless of the choice of $h$, by dimensional analysis the finite part of the integral 
$\lim _{x^{2} \rightarrow 0} e^{i q_{2} x^{2}} \int d p_{2} h\left(p_{2}\right) e^{-i p_{2} x^{2}}$ would have to be proportional to $q_{2}$, so our answer would be the unchanged up to a redefinition of $\lambda$ by a numerical factor, assuming $h\left(p_{2}\right)$ is real and reasonably well-behaved.

Using (6.24) and (6.25), the other components of the exact propagator are:

$$
\begin{aligned}
& \left\langle\psi_{+}(q) \psi_{-}^{\dagger}\left(q^{\prime}\right)\right\rangle=\frac{-q_{2}(1-\lambda)}{2 q_{-}} N \theta\left(q_{-}\right)(2 \pi)^{2} \delta^{2}\left(q+q^{\prime}\right) \\
& \left\langle\psi_{-}(q) \psi_{+}^{\dagger}\left(q^{\prime}\right)\right\rangle=\frac{-q_{2}(1+\lambda)}{2 q_{-}} N \theta\left(q_{-}\right)(2 \pi)^{2} \delta^{2}\left(q+q^{\prime}\right) \\
& \left\langle\psi_{+}(q) \psi_{+}^{\dagger}\left(q^{\prime}\right)\right\rangle=\frac{q_{2}^{2}\left(1-\lambda^{2}\right)}{2 \sqrt{2} q_{-}^{2}} N \theta\left(q_{-}\right)(2 \pi)^{2} \delta^{2}\left(q+q^{\prime}\right)
\end{aligned}
$$

Let us compare with the equal time propagator derived earlier in section 2 , obtained from 2.23$)$ :

$$
\begin{aligned}
& \lim _{x^{+} \rightarrow 0^{+}} \int \frac{d k_{+}}{2 \pi}\left(\langle\psi(k) \bar{\psi}(k)\rangle \gamma^{0} e^{i k_{+} x^{+}}\right) \\
& =\lim _{x^{+} \rightarrow 0^{+}} \int \frac{d k_{+}}{2 \pi} \frac{-i k_{\mu} \gamma^{\mu}-i \Sigma(k)_{+} \gamma^{+}+\Sigma_{I}(k)}{k^{2}+i \epsilon} \gamma^{0}(2 \pi)^{2} \delta^{2}\left(k+k^{\prime}\right)
\end{aligned}
$$

Comparison with $(6.23)-6.28)$, immediately implies that $f_{0}^{2}+g_{0}=0$, and $f_{0} \sim \lambda$. We see that, because of the $\lambda$ independence of the vacuum, all $\lambda$ dependence arises from the fermionic constraints 6.5, 6.6.

Note that in this discussion we did not use dimensional regularization which was used in the Euclidean calculation and hence this agreement is significant.

\subsection{Large $N$ perturbation theory and a formal large $N$ solution}

Now that we have the classical solution at large $N$, we can construct a $W_{\infty}$ covariant perturbation theory by parameterizing

$$
M=e^{-i W / \sqrt{N}} M_{\text {class. }} e^{i W / \sqrt{N}}
$$

in terms of the fluctuations $W$.

The $W$ 's satisfy an algebra inherited from the commutation relations of the $M$ 's, and break up into subalgebras when expanded around $M_{\text {class. }}$ exactly as in [47], where a similar treatment was carried out for $Q C D_{2}$ that gave rise to 't Hooft's well known equation for the spectrum of mesons. 
The fluctuation equation obtained from substituting 6.30 into $-i \partial_{+} M=[H, M]$ is a second order integral equation for $W$. It takes the following schematic form:

$$
\begin{aligned}
\partial_{+} \bar{W}\left(p, p^{\prime} ; x^{+}\right)= & \frac{i}{4}\left(\frac{p_{2}^{\prime 2}+m^{2}}{p_{-}^{\prime}}-\frac{p_{2}^{2}+m^{2}}{p_{-}}\right) \bar{W}\left(p, p^{\prime} ; x^{+}\right) \\
& +\lambda \int d^{2} q(\ldots W)+\lambda^{2} \int d^{2} q d^{2} q^{\prime}(\ldots W)
\end{aligned}
$$

where $\bar{W}\left(p, p^{\prime} ; x^{+}\right)=W\left(p, p^{\prime}\right) \theta\left(p_{-}\right)$

Any single-trace operator $\left(J_{0}, J_{1}, J_{2}\right.$ etc. $)$ can be expressed in terms of $M$ 's using solution to the constraints, 6.4), 6.24) and (6.25), and Hamilton's equation for $\partial_{+} \psi_{-}$; hence, any two-point function of $J$ 's can be expressed in terms of two-point functions of $M$ 's. Because $\langle M(p, p+k)\rangle=0$ for nonzero $k$, the Green's function for the fluctuation equation 6.30 is needed to calculate the leading nonzero contribution to a generic two-point function of $M$ 's in the $1 / N$ expansion. Thus, solving equation 6.31 for $W_{\infty}$-covariant fluctuations around the classical solution would, in principle, enable one to calculate exact two-point functions of all single trace operators in the large $N$ limit (subject to, of course, subtleties associated with the light-cone Hamiltonian formalism.)

\section{Comments on the Holographic Dual}

\subsection{Vector models are dual to higher spin gauge theories}

The non-renormalization of single trace currents in $U(N)$ Chern-Simons-fermion theory at leading order in $1 / N$ indicates that its holographic dual has a free spectrum of purely higher spin gauge fields, of spin $s=0,1,2,3, \cdots$, and their bound states (which are dual to multi-trace operators). The divergences of the single trace currents are mixed with double and possibly triple trace operators, and the mixing is suppressed by $N^{-\frac{1}{2}}$. By comparing two and three point functions of the currents, we see that $N^{-\frac{1}{2}}$ should be identified with the bulk coupling constant.

Classically, the bulk nonlinear equations of motion must therefore preserve the higher spin gauge symmetries as well, for otherwise they would introduce extra longitudinal degrees of freedom for the higher spin fields even in the zero coupling limit. This means that the dual bulk theory is a pure higher spin gauge theory. On the other hand, the higher spin symmetry in the Chern-Simons-fermion theory is broken by $1 / N$ corrections, and we expect the HS gauge symmetry to be broken in the bulk by boundary conditions through loop effects ${ }^{22}$ and the HS gauge fields become massive through

\footnotetext{
${ }^{22}$ This has been shown to occur, for instance, in Vasiliev's A-type theory in $A d S_{4}$ with the $\Delta=2$ boundary condition, dual to the critical $O(N)$ model 33 .
} 
the mixing of its longitudinal mode with bound states.

Our argument of non-renormalization of singlet bilinear currents extends to general vector models coupled to Chern-Simons gauge fields, such as the various supersymmetric extensions of the Chern-Simons-fermion theory discussed so far. We therefore anticipate all such theories to be dual to some higher spin gauge theory in the bulk. The bulk theory is generally not parity invariant.

\subsection{Parity violating Vasiliev theory}

More precisely, we conjecture that the three-dimensional critical $U(N)$ Chern-Simons theory at level $k$ coupled to a single fundamental fermion in the 't Hooft limit $N \gg 1$, $\lambda=N / k$ finite, is dual to a parity-violating higher spin gauge theory in $A d S_{4}$ that contains one gauge field for each non-negative integer spin $s=0,1,2, \cdots$. A class of such theories has indeed been constructed by Vasiliev, generalizing the parity preserving A-type and B-type theories (which have been conjectured to be dual to free/critical $U(N)$ scalar and fermion vector models in the singlet sector). We will refer to them as parity-violating Vasiliev theories in $A d S_{4}$. The bulk interactions of the parity violating Vasiliev theory are governed by a single function

$$
f(X)=1+X \exp (i \theta(X))
$$

where $\theta(X)$ is a real, even function in $X$, but otherwise unconstrained (in the classical theory). Roughly, when expanded in powers of $X$, the order $X^{n}$ term in $f(X)$ governs the $(n+2)$-th order coupling of the bulk theory.

Vasiliev's theory has the feature that the bulk scalar field has mass $m^{2}=-2 / \ell_{A d S}^{2}$, and the dual scalar operator (with one choice of boundary condition) has classical dimension 2. This does not follow from the linearized higher spin gauge symmetry, and is required in order to construct higher spin gauge invariant interactions. This feature is in precise agreement with the non-renormalization of the scalar operator $\bar{\psi} \psi$ at the planar level in the Chern-Simons-fermion vector model, which we have argued using the anomalous current conservation relation and verified explicitly at two-loop.

Now let us discuss the parity transformation in Vasiliev theory. The function $f(X)$ transforms to its complex conjugate $\overline{f(X)}$ under parity. One may either assign even or odd parity to the bulk scalar field, which corresponds to assigning even or odd parity to the variable $X$. In the former case, a parity invariant theory is given by $f(X)=\overline{f(X)}$, or $f(X)=1+X$; this is the A-type theory. In the latter case, where $X$ is parity odd, a parity invariant theory is given by $f(X)=1+i X$; this is the B-type theory. The B-type theory has been conjectured to the dual to the free limit of the $U(N)$ ChernSimons-fermion theory, where the Chern-Simons gauge fields decouple and their only 
effect is to restrict the operator spectrum to gauge singlets; parity is restored in this free limit.

It is thus natural to suspect that the Chern-Simons-fermion theory at large $N$ but finite 't Hooft coupling $\lambda=N / k$, is dual to the parity violating Vasiliev theory governed by a function $f(X)=1+X \exp (i \theta(\lambda, X))$, where

$$
\theta(\lambda, X)=\frac{\pi}{2}+\beta(\lambda, X)
$$

is real, even in $X$, and depends on $\lambda$ in some way. For instance, one should have $\beta(0, X)=0$, so that in the $\lambda=0$ case the conjecture reduces to the duality between $\mathrm{B}$ type theory and free fermions. While the CS-fermion theory violates parity symmetry, parity is restored if we flip the Chern-Simons level $k$ (i.e. $\lambda \rightarrow-\lambda$ ) together with the standard parity action on the fields, under which the scalar operator $\bar{\psi} \psi$ is parity odd. So if the Chern-Simons-fermion theory is dual to the parity violating Vasiliev theory, we must also have $\beta(-\lambda, X)=-\beta(\lambda, X)$.

While there is a priori an infinite family of parity-violating Vasiliev theories in $A d S_{4}$ governed by the function $f(X)$, we may speculate that there is a particular choice of $f(X)$ determined by the 't Hooft coupling $\lambda$ in the above form, that describes the bulk theory dual to critical $U(N)$ CS-fermion theory at large $N$. It is then an interesting question to determine $\theta(\lambda, X)$ as a function of $\lambda$. In principle, this can be done perturbatively by comparing the $n$-point functions of the HS currents in CSfermion theory and the bulk Vasiliev theory. We may expand the function $\beta(\lambda, X)$ as $\sum_{k=0}^{\infty} \beta_{k}(\lambda) X^{2 k}$. Then $\beta_{k}$ may in principle be determined by comparing with the $(2 k+3)$-point function of the higher spin currents in the CFT at leading order in $1 / N$. In particular, the leading phase $\beta_{0}(\lambda)$ can be determined by comparing with the three-point functions of currents.

The simplest check of such a conjecture would be the comparison of bulk tree level three point functions in parity-violating Vasiliev theory, and the planar three point function $\langle J J J\rangle$ in the CS-fermion theory. The former computation, performed along the lines of [32], will be presented elsewhere. The result obtained using the gauge function method of [32] (" $W=0$ gauge") indeed agrees with the parity-even part of $\langle J J J\rangle$ in CS-fermion theory up to two-loop: in particular, the property that $c_{B}$ in (4.34) is independent of the three spins is a prediction from the bulk theory! However, we have failed to produce the parity-odd structure of $\langle J J J\rangle$ from the bulk Vasiliev theory. This could be either due to the singular nature of the $W=0$ gauge in [32 and that the parity-odd contribution is simply missed by the regularization of [32], or it could be that the correct dual bulk theory to the CS-fermion CFT is not described by Vasiliev's system, but rather some other higher spin gauge theory with the same field content in $A d S_{4}$. We shall leave the resolution of this puzzle to future work. 
Let us comment on higher spin symmetry breaking as seen from the correlation functions. While at tree level in the bulk, we expect the higher spin symmetry to be preserved, there can be subtle boundary contributions in computing correlators. Examples of similar nature in the bulk dual of critical $O(N)$ model have been discussed in [33]. Recall the anomalous current conservation relation in the boundary theory, of the form

$$
\partial^{\mu} j_{\mu \cdots}^{(s)} \sim \frac{1}{\sqrt{N}} \sum_{s_{1}+s_{2}<s}\left[j^{\left(s_{1}\right)}\right] \otimes\left[j^{\left(s_{2}\right)}\right]+\frac{1}{N} \sum_{s_{1}+s_{2}+s_{3}<s}\left[j^{\left(s_{1}\right)}\right] \otimes\left[j^{\left(s_{2}\right)}\right] \otimes\left[j^{\left(s_{3}\right)}\right]
$$

On the right hand side, we have listed the conformal families that can appear in the divergence of the current $j^{(s)}$. The dependence on 't Hooft coupling $\lambda$ is omitted in this expression. As discussed in section 3, though these multi-trace operators appear to be suppressed by powers of $1 / N$, they can contribute to the corresponding divergence of correlation functions at the leading nontrivial order in $1 / N$.

For the three-point functions $\left\langle j^{\left(s_{1}\right)} j^{\left(s_{2}\right)} j^{\left(s_{3}\right)}\right\rangle$, the naive current conservation equation at leading order in $1 / N$, namely $\mathcal{O}\left(N^{-\frac{1}{2}}\right)$ when the two point functions are normalized to $\mathcal{O}\left(N^{0}\right)$,

$$
\partial^{\mu_{1}}\left\langle j_{\mu_{1} \cdots}^{\left(s_{1}\right)} j^{\left(s_{2}\right)} j^{\left(s_{3}\right)}\right\rangle=0
$$

is necessarily satisfied if $s_{1}<s_{2}+s_{3}{ }^{23}$

On the other hand, if $s_{1} \geq s_{2}+s_{3}$, then there is the possibility of a nontrivial $\mathcal{O}\left(N^{-\frac{1}{2}}\right)$ contribution,

$$
\partial^{\mu_{1}}\left\langle j_{\mu_{1} \ldots}^{\left(s_{1}\right)} j^{\left(s_{2}\right)} j^{\left(s_{3}\right)}\right\rangle \sim \frac{1}{\sqrt{N}}\left\langle j^{\left(s_{2}\right)} j^{\left(s_{2}\right)}\right\rangle\left\langle j^{\left(s_{3}\right)} j^{\left(s_{3}\right)}\right\rangle
$$

So even at leading order in $1 / N$, the naive current conservation relation is violated by the three point function. Naively, the boundary-to-bulk propagator of the higher spin fields in $A d S_{4}$ is divergence free with respect to the position and polarization of the source current, and it may seem that such a non-conserved three point function cannot result from a tree level bulk computation. As pointed out in [33], however, this is not necessarily the case, due to subtle boundary contributions to the bulk integral.

\footnotetext{
${ }^{23}$ In the explicit computations we have performed for the classical divergence of currents for spins up to 5 we in fact find that this the current is effectively conserved in correlators provided $s_{1}<s_{2}+s_{3}+2$. It is possible that this relation is more generally true and can be proved from an analysis of structures allowed by conformal invariance in 3 point functions. If this is the case the three point function will be divergence free with respect to all three currents if the three spins $s_{1}, s_{2}, s_{3}$ obey this less strict triangular inequality.
} 


\subsection{A higher spin gauge theory as a string theory}

A long standing question is whether the pure higher spin gauge theory in $\operatorname{AdS}$ can be embedded in string theory. We have seen that quite generally vector models coupled to Chern-Simons fields are dual to higher spin gauge theories. On the other hand, a large class of supersymmetric quiver Chern-Simons-matter theories are dual to string theories in $A d S_{4}$. A particularly interesting class of examples is that of the ABJ theory [12]: the $\mathcal{N}=6 U(N)_{k} \times U(M)_{-k}$ Chern-Simons theory coupled to a pair of bifundamental hypermultiplets. It has been conjectured to be dual to type IIA string theory on $A d S_{4} \times \mathbb{C P}^{3}$, with a flat $B$-field obeying

$$
\frac{1}{2 \pi \alpha^{\prime}} \int_{\mathbb{C P}^{1}} B=\frac{N-M}{k}+\frac{1}{2},
$$

when $|N-M|<k$. The need for the shift by $\frac{1}{2}$ in the equation above was explained in 55$]{ }^{24}$

While the dual string theory is weakly coupled in the 't Hooft limit, the duality is believed to hold exactly for all $N, M$ and $k$. In the special case $M=1$ (or generally small $M$ ), ABJ theory reduces to a supersymmetric Chern-Simons vector model, in the following sense. The most straightforward $\mathcal{N}=3$ supersymmetric generalization of the vector model we considered so far is the $\mathcal{N}=3 U(N)$ or $S U(N)$ Chern-Simons theory coupled to a fundamental hypermultiplet. Most features of large $N$ vector models discussed in this paper still hold when a number of fundamental flavors are introduced (the number of flavors does not scale with $N$ in the large $N$ limit here). Now consider $\mathcal{N}=3 U(N)_{k}$ Chern-Simons theory with $2 M$ hypermultiplets. By essentially the same argument as in section 2, at large $N$ this vector model is dual to a higher spin gauge theory in $A d S_{4}$ with $U(2 M)$ Chan-Paton factors ${ }^{25}$ To obtain the $\mathcal{N}=6$ theory, one needs to further gauge the $U(M)$ flavor symmetry with $\mathcal{N}=3$ Chern-Simons coupling at level $-k$ (as opposed to level infinity). This however corresponds to simply modifying the $A d S$ boundary condition on the bulk $U(M)$ spin-1 gauge fields, from the purely magnetic boundary condition to an electric-magnetic mixed boundary condition [62].

Let us comment briefly on the distinction between $U(N)$ and $S U(N)$ gauge groups here. Take the ABJ theory with $M=1$ as an example. The gauge group is $U(N)_{k} \times$ $U(1)_{-k}$. Denote by $a$ and $\tilde{a}$ the diagonal $U(1)$ of the $U(N)_{k}$ and the $U(1)_{-k}$ gauge fields, respectively. Note that $a$ has Chern-Simons level $N k$ as a $U(1)$ gauge field. The

\footnotetext{
${ }^{24}$ We thank O. Aharony for bringing this to our attention.

${ }^{25}$ Note that Vasiliev's system can be generalized to a theory of nonabelian higher spin fields by introducing $U\left(N_{f}\right)$ Chan-Paton factors, i.e. replacing the $*$ algebra by its tensor product with the algebra of $N_{f} \times N_{f}$ matrices.
} 
matter fields couple to $b=a-\tilde{a}$ only. The Chern-Simons action for $a, \tilde{a}$ takes the form

$$
\frac{k}{4 \pi} \int(N a \wedge d a-\tilde{a} \wedge d \tilde{a})=\frac{k}{4 \pi} \int(N a \wedge d a-(a-b) \wedge d(a-b)) .
$$

Since only $b$ couple to the matter fields, we can integrate out $a$ exactly, which sets $a=-\frac{1}{N-1} b$. The resulting Chern-Simons action is that of the $U(1)$ gauge field $b$ at

level $-\frac{N}{N-1} k$. So the same theory can be expressed as $S U(N)_{k} \times U(1)_{-k^{\prime}}$ Chern-Simons coupled to 2 bifundamental hypermultiplets, where $k^{\prime}=\frac{N}{N-1} k$.

We are now naturally led to the conjecture that the $U(N) \times U(M)$ ABJ theory in the vector model limit, i.e. large $N$ and fixed finite $M$, is dual to an $\mathcal{N}=6$ version of the parity violating Vasiliev theory with $U(M)$ Chan-Paton factors. Furthermore, we conjecture that the type IIA string field theory on $A d S_{4} \times \mathbb{C P}^{3}$, with a flat $B$-field as in (7.6), in limit $N \gg M$, is a higher spin gauge theory. Note that in the vector model limit $N \gg M$, the 't Hooft coupling $N / k$ is restricted to be less than 1 , and the bulk IIA string theory is always in the highly stringy regime.

In the ABJ theory, the bulk single string states are dual to single trace operators, of the form

$$
\operatorname{Tr}(A B A B \cdots)
$$

where $A$ and $B$ denote fields in the $(\square, \bar{\square})$ and $(\bar{\square}, \square)$ representations respectively. In the $M=1$ case, the single trace trace breaks into the product of a number of bilinears which are singlets under the $S U(N)$ gauge group. So we learn that the general single string state should correspond a multi-particle state of higher spin fields. It suggests that the higher spin gauge fields are in fact more fundamental than strings in this case, as the latter are composite objects made out of the former! Note that the coupling constant $g$ of the higher spin gauge theory is mapped to $\sim 1 / \sqrt{N}$ of the vector model. Now $\lambda_{\text {bulk }}=g^{2} M \sim M / N$ is the bulk 't Hooft coupling. We see that the vector model limit $N \gg M$ is precisely where the nonabelian higher spin gauge theory is weakly coupled. As $M / N$ becomes of order 1, the higher spin fields interact strongly, and we expect their bound states to form the string excitations. If the dual higher spin gauge theory is indeed described by Vasiliev's system, it would be very interesting to find the explicit map from Vasiliev's master fields to the (closed) string fields, which could allow for a precise formulation of the closed string field theory in this limit.

\section{Discussion}

This paper has been devoted to the study of $U(N)$ Chern-Simons theory coupled to a single massless fundamental fermion. We set out to to solve this theory in the 't 
Hooft large $N$ limit and have been partly successful in this task. In particular, we were able to compute the partition function on $\mathbb{R}^{2}$ of the theory at finite temperature at all values of the 't Hooft coupling. In order to perform this computation we employed a lightcone gauge, and used a dimensional reduction regularization scheme. It would be useful to perform further checks of the consistency of our gauge and regularization scheme.

In the course of our analysis of this theory we have encountered the fact that the higher spin currents obey anomalous conservation equations. In the classical theory we have computed the explicit form of these conservation equation at low values of the spin (see e.g. (3.19)). These nonlinear anomalous conservation equations contain a large amount of information; for example they encode the anomalous dimensions of the spin $s$ currents in a $\frac{1}{N}$ expansion (see subsection 3.4). It would be interesting to determine the explicit form of these anomalous conservation equations, if possible, as a function of $\lambda$.

We have also argued that the spectrum of local gauge invariant operators in our theory, at dimensions of order unity, is not renormalized as a function of the 't Hooft coupling at leading order in large $N$. A class of such operators (those that may be written entirely in terms of $x^{-}$derivatives) are dressed due to interactions only by rainbow graphs in the large $N$ limit. The "quantum dressed" operator obtained in this way is given by the solution to an integral equation both at zero and at finite temperature. Exact expressions for all two and and three point functions are obtained by sewing these quantum dressed operators together using the exact fermion propagator computed in this paper. Such a solution would allow us to find exact expressions, for example, of fermion number current and stress tensor two point functions at finite temperature as a function of $\vec{k}$ and $\omega$. As these quantities are of obvious physical interest, the corresponding integral equations deserve serious attention.

In this paper we have analyzed in detail the spectrum of local operators. But clearly the theory also admits interesting non-local gauge invariant observables, namely the Wilson loop operators specified by a choice of a closed contour in space-time and a representation of the gauge group. In pure Chern-Simons theory, these are topological observables which compute certain knot invariants [3]. When Chern-Simons theory is coupled to matter, the Wilson loops are no longer topological. In a theory with fundamental matter, however, at leading order at large $N$ their expectation values are the same as in pure Chern-Simons theory, since diagrams with fundamental matter loops are suppressed. It may be interesting to explicitly compute the leading $1 / N$ corrections for some Wilson loop in our theory. In standard examples of AdS/CFT, Wilson loop operators are dual to macroscopic open strings ending on the closed contour at the boundary. In this paper we have proposed that our theory should be dual to some 
higher spin gauge theory in $A d S_{4}$. It would be interesting to understand if Vasiliev's theory, or a deformation thereof, may possibly contain extended objects which could be dual to the Wilson loops of the gauge theory.

It has recently been realized that the partition functions of supersymmetric ChernSimons theories on $S^{3}$ are often exactly computable via the techniques of supersymmetric localization [56 59]. This quantity appears to play the role of a $c$ function under the renormalization group flow and so is of clear physical interest. It seems possible that the techniques employed in this paper will allow the exact computation of the $S^{3}$ partition function of our non-supersymmetric theory in the large $N$ limit, as a function of $\lambda$.

As we have explained in subsection 3.1 .3 above, in the limit $\lambda \rightarrow 0$, the partition function of our theory on $S^{2} \times S^{1}$ undergoes an interesting phase transition at a temperature of order $\mathcal{O}(\sqrt{N})[39$. The low temperature phase may be thought of as a gas of single traces. At temperatures that are held fixed when $N$ is taken to infinity, the single trace anomalous dimension non renormalization theorems of this paper demonstrate that the free energy of this phase is not renormalized as a function of $\lambda$. At temperatures at of order $\sqrt{N}$ the free energy of of the low temperature phase presumably does get renormalized as a function of $\lambda$. And the free energy of the high temperature phase of the partition function (which be thought of as a gas of the fundamental fermions rather than their mesonic bound states) certainly does get renormalized a function of $\lambda$. In this paper in particular we have computed the coefficient of the $T^{3}$ growth of the free energy in the very high temperature limit, and have shown that this coefficient, which is nonzero at generic $\lambda$, vanishes in the limit $\lambda \rightarrow 1$. It would be very interesting to understand how the competition between the two phases changes as a function of $\lambda$, especially in the limit $\lambda \rightarrow 1$. Does the vanishing of the free energy at extremely high temperatures, for instance, suggest that the phase transition temperature between the low and high temperature phases diverges as $\lambda \rightarrow 1$ ?

It would also be interesting to investigate transport properties in the high temperature phase. The following argument suggests that the mean free path of any particular fermionic quantum, is of order $N$. Consider, for instance, a fermion of colour $i$ scattering off an anti fermion of colour $i$ to yield a fermion - anti-fermion pair of colour $j$. This scattering amplitude is of order $\frac{1}{N}$, as a consequence of which the scattering probability is of order $\frac{1}{N^{2}}$. The number of potential scattering partners is of order unity in the thermal bath, while there are $N$ possibilities for the colour $j$ of the final fermion pair. It follows that the probability that any given fermionic quantum undergoes a scattering process is of order $\frac{1}{N}$, giving rise to a mean free path of order $N$. This result should apply at every value of $\lambda$. All this very similar to the known behavior of transport properties in the critical $\mathrm{O}(\mathrm{N})$ model (see e.g. [22]) and very 
different from theories with matrix degrees of freedom in the t'Hooft limit where the mean free time between collisions of a single parton is independent of $N$ [60]. The fact that the fermion gas effectively behaves as a free gas as far as transport properties are concerned, suggests that fluctuations about the high temperature phase do not decay over a time of order unity. This discussion suggests that large $N$ vector models in the 't Hooft limit thermalize much less efficiently than their matrix model counterparts. For this reason the dual description of such a high temperature phase has properties that are very different from a conventional classical black hole. ${ }^{26}$

It should also be possible to generalize our discussion of the finite temperature behaviour of our theory to include a chemical potential for fermion number. The finite $\lambda$ behaviour of such a system describes an interacting Fermi sea in three dimensions, and so may be of interest for various condensed matter problems.

It would be interesting to generalize our computation of the finite temperature free energy to Chern-Simons theory interacting with fundamental bosons [37], and also to the supersymmmetric theory with different numbers of supersymmetries.

An outstanding question about the theory studied in this paper as well as the generalizations described above is "what is its bulk dual description?" We have argued above that this dual description is a higher spin theory. However we do not yet have a precise conjecture for the nature of this dual; this is clearly an issue of immediate interest, especially since the maximally supersymmetric version of our theory is embedded into string theory as a limit of the ABJ construction (see subsection 7.3).

In this paper we have studied only the 't Hooft large $N$ limit of our theory. It is however clear from the example of ABJM theory, that large $N$ Chern Simons theories admit a wider class of large $N$ limits (e.g. $N \rightarrow \infty, \lambda \rightarrow \infty, \frac{N}{\lambda}$ fixed in the ABJM context). It would be interesting to investigate whether the theory studied in this paper admits an analogous double scaling limit, in which $\lambda$ is scaled to unity in a manner coordinated with the large $N$ limit 27 . The dimensions of the higher spin currents could be renormalized as a function of coupling in such a limit. The fact that we have been able to obtain exact results as a function of $\lambda$ perhaps makes such an investigation feasible.

To end this discussion we recall we could very easily argue that the spectrum of

\footnotetext{
${ }^{26}$ When the ranks, $N_{1}$ and $N_{2}$ of the ABJ theory are both comparable, the theory undergoes a deconfinement transition, dual to a Hawking Page transition, at a temperature of order unity. The high temperature phase in this transition is a black hole in Einstein gravity. It follows from the discussion above, on the other hand, that when $N_{1}$ is lowered to a number of order unity with $N_{2}$ still large then the deconfinement phase transition temperature is much larger (it scales like $\sqrt{N_{2}}$ ). Moreover the dual black hole appears to change its nature qualitatively; it appears to stop absorbing.

${ }^{27}$ We thank O. Aharony for discussions on this point.
} 
'single trace' operators in our theory is not renormalized as a function of $\lambda$, in the 't Hooft large $N$ limit, by combining conformal representation theory with the sparseness of the single trace spectrum in vector models. The same non-renormalization argument does not apply in theories with adjoint or bifundamental matter fields as the single trace spectrum of these theories is not sparse ${ }^{28}$ On the other hand, as we have explained in the introduction, non supersymmetric effective large $N$ Chern-Simons fixed lines are easily constructed with matter fields in adjoint or bifundamental representations. The scaling dimension of single trace operators in such theories is protected neither by conformal nor supersymmetric representation theory. In these theories all single trace operators baring the stress tensor and currents for global symmetries can, and presumably do get renormalized. It is at least conceivable that some theory with adjoint or bifundamental fermions admits a strong coupling limit in which all but a finite number of single trace operators are infinitely renormalized, and the dual description of the theory is Einstein gravity in $A d S_{4}$, coupled to a minimal number of additional fields. It would clearly be very interesting to identify any theory with this property.

As is clear from the discussion above, the computations presented in this paper have merely scratched the surface of a large and potentially very interesting area of investigation. We hope to report on some of the topics discussed above in the future.

\section{Acknowledgements}

We are grateful to Ofer Aharony and Rajesh Gopakumar for crucial discussions that led to the formulation of some of the questions addressed in this paper. We would like to acknowledge Sayantani Bhattacharyya, Jyotirmoy Bhattacharya and V. Umesh for collaboration at various stages of this project. We thank O. Aharony and J. Bhattacharya for comments on the manuscript. We would also like to acknowledge useful discussions with C.-M. Chang, K. Damle, J. David, A. Dhar, S. Dutta, D. Hofman, N. Iizuka, D. Jafferis, S. Kim, S. S. Lee, G. Mandal, K. Papadodimas, M. Rangamani, S. Sachdev, A. Sen, C. Sonnenshein and E. Witten. S.G. is supported by Perimeter Institute for Theoretical Physics. Research at Perimeter Institute is supported by the Government of Canada through Industry Canada and by the Province of Ontario through the Ministry of Research \& Innovation. The work of S.M. was supported by a Swarnajayanti fellowship. The work of S.M., S.P., S.T. and S.W. was supported by the DAE, government of India. S.G. would like to thank Princeton U., the Simons Center for Geometry and Physics, Mc Gill U., the Weizmann Institute, the organizers of the

\footnotetext{
${ }^{28}$ In supersymmetric theories with such matter content, supersymmetric indices and representation theory give rise to a new source of non-renormalization theorems for single trace operators even in theories with bifundamental and adjoint matter, see e.g. the paper 61].
} 
PhyMSI conference in Cargese and of the Simons Summer Workshop in Mathematics and Physics 2011 for hospitality during completion of this work. S.M. would like to thank the Weizmann Institute, Cambridge University, KIAS, ICTP, the organizers of GR19, PASCOS 2010, and the Indian Strings Meeting (ISM) 2011 for for their hospitality while this work was in progress. S.P. would like to thank the organizers of ISM 2011, and the Centre for High Energy Physics, Indian Institute of Science, Bangalore for their hospitality. X.Y. would like to thank the organizers of GR19 and of ISM 2011, Tata Institute of Fundamental Research, Berkeley Center for Theoretical Physics, Simons Center for Geometry and Physics, Fields Institute, and Aspen Center for Physics for their hospitality. The work of X.Y. was supported by the Fundamental Laws Initiative Fund at Harvard University and in part by NSF Award PHY-0847457. S.M., S.P., S.T. and S.W. would also like to acknowledge our debt to the people of India for their generous and steady support to research in the basic sciences.

\section{Appendices}

\section{A Conventions for Propagators and Gauge Condi- tions}

The Euclidean action for our theory is

$$
S=\frac{i k}{4 \pi} \int \operatorname{Tr}\left(A d A+\frac{2}{3} A^{3}\right)+\int \bar{\psi} \gamma^{\mu} D_{\mu} \psi
$$

where

$$
D_{\mu} \psi=\partial_{\mu} \psi-i A^{a} T^{a} \psi
$$

We have

$$
\begin{aligned}
\epsilon_{123} & =\epsilon^{123}=1, \\
\gamma^{i} & =\sigma^{i}, \quad(i=1 \ldots 3) \\
\bar{\psi}^{\alpha} & =\left(\psi_{\alpha}\right)^{*},
\end{aligned}
$$

where $\sigma^{i}$ are the ordinary Pauli matrices. Note then that all $\gamma^{\mu}$ are Hermitian. This implies that $\bar{\psi} \psi$ and $\bar{\psi} \gamma^{\mu} \psi$ are real, while $\int d x \bar{\psi} \gamma^{\mu} \partial_{\mu} \psi$ is imaginary. The gauge field will be taken to be $A^{a} T_{a}$ where $T_{a}$ is the fundamental generator normalized so that $\operatorname{Tr} T_{a}^{2}=\frac{1}{2}$. Note that

$$
\sum_{a}\left(T^{a}\right)_{m}^{n}\left(T^{a}\right)_{p}^{q}=\frac{1}{2} \delta_{m}^{q} \delta_{p}^{n}
$$




\section{A.1 Lightcone gauge}

Let us define

$$
\begin{aligned}
& x^{ \pm}=\frac{x^{1} \pm i x^{2}}{\sqrt{2}}, \\
& A^{ \pm}=A_{\mp}=\frac{A^{1} \pm i A^{2}}{\sqrt{2}}, \\
& p^{ \pm}=p_{\mp}=\frac{p^{1} \pm i p^{2}}{\sqrt{2}}, \\
& p_{s}^{2} \equiv p_{1}^{2}+p_{2}^{2}=2 p^{+} p^{-} .
\end{aligned}
$$

We define the lightcone gauge in Euclidean signature by the condition $A_{-}=0$. This can be obtained from Wick rotation of the standard lightcone gauge in Lorentzian signature. However, in this paper we often think of $x^{1}, x^{2}$ as purely spatial coordinates. In particular, in the finite temperature calculation, the thermal time direction is orthogonal to the complex lightcone direction.

Note that under a rotation in the 12 plane $A_{-} \rightarrow e^{i \alpha} A_{-}$. Consequently rotations in the 12 plane commute with the condition $A_{-}=0$.

Defining the momentum space fields

$$
\begin{aligned}
A_{\mu}(x) & =\int \frac{d^{3} p}{(2 \pi)^{3}} e^{i p \cdot x} A_{\mu}(p), \\
\psi_{\alpha}(x) & =\int \frac{d^{3} p}{(2 \pi)^{3}} e^{i p \cdot x} \psi_{\mu}(p), \\
\bar{\psi}^{\alpha}(p) & =\left(\psi_{\alpha}(-p)\right)^{*},
\end{aligned}
$$

the momentum space action in lightcone gauge is

$$
\begin{aligned}
S & =\frac{-i k}{2 \pi} \int \frac{d^{3} p}{(2 \pi)^{3}} \operatorname{Tr} A_{3}(-p) p_{-} A_{+}(p)+\int \frac{d^{3} p}{(2 \pi)^{3}} i \bar{\psi}(-p)\left(\gamma^{\mu} p_{\mu}+M_{\text {bare }}\right) \psi(p) \\
& -i \int \frac{d^{3} p}{(2 \pi)^{3}} \int \frac{d^{3} q}{(2 \pi)^{3}} \bar{\psi}(-p)\left(\gamma^{+} A_{+}(-q)+\gamma^{3} A_{3}(-q)\right) \psi(p+q),
\end{aligned}
$$

where $A=A^{a} T_{a}$. 
It follows from this action that 29

$$
\begin{aligned}
& \left\langle\psi(p)_{n} \bar{\psi}^{m}(-q)\right\rangle=\delta_{n}^{m} \frac{-i \gamma^{\mu} p_{\mu}}{p^{2}} \times(2 \pi)^{3} \delta(p-q) \\
& \left\langle A_{3}^{a}(p) A_{+}^{b}(-q)\right\rangle=-\left\langle A_{+}^{b}(p) A_{3}^{a}(-q)\right\rangle=-\frac{4 \pi i}{k} \frac{1}{p^{+}} \times(2 \pi)^{3} \delta(p-q) \delta^{a b} .
\end{aligned}
$$

Adopting the notation

$$
\left\langle A_{\mu}^{a}(p) A_{\nu}^{b}(-q)\right\rangle=(2 \pi)^{3} \delta(p-q) G_{\mu \nu}(p) \delta^{a b},
$$

we have

$$
G_{+3}(p)=-G_{3+}(p)=\frac{4 \pi i}{k p^{+}}
$$

In coordinate space, the gauge propagator reads

$$
\begin{aligned}
& \left\langle A_{3}(x) A_{+}(0)\right\rangle=-\left\langle A_{+}(x) A_{3}(0)\right\rangle=-\frac{8 \pi i}{k} \int \frac{d^{3} p}{(2 \pi)^{3}} \frac{p^{-}}{p_{s}^{2}+i \epsilon} e^{i p \cdot x}=-\frac{8 \pi}{k} \delta\left(x^{3}\right) \partial_{+} \int \frac{d^{2} p_{s}}{(2 \pi)^{2}} \frac{e^{i p_{s} \cdot x}}{p_{s}^{2}+i \epsilon} \\
& =\frac{2}{k} \frac{\delta\left(x^{0}\right)}{x^{+}} .
\end{aligned}
$$

\section{A.2 Temporal gauge}

The temporal gauge is defined by the condition $A_{3}=0$ (Wick rotating the Lorentzian temporal gauge $A_{0}=0$ ). In this gauge, the gauge field propagator is written in position space as

$$
\left\langle A_{i}(x) A_{j}(0)\right\rangle=\frac{2 \pi i}{k} \epsilon_{i j} \operatorname{sign}\left(x^{3}\right) \delta^{2}(\vec{x}),
$$

and in momentum space,

$$
\begin{aligned}
\left\langle A_{i}(p) A_{j}(-q)\right\rangle & =\frac{2 \pi}{k} \epsilon_{i j}\left[\frac{1}{p^{3}+i \epsilon}+\frac{1}{p^{3}-i \epsilon}\right](2 \pi)^{3} \delta^{3}(p-q) \\
& =\frac{4 \pi}{k} \epsilon_{i j} \frac{p^{3}}{\left(p^{3}\right)^{2}+\epsilon^{2}}(2 \pi)^{3} \delta^{3}(p-q) .
\end{aligned}
$$

${ }^{29}$ The propagator for a theory whose Euclidean action is

$$
S=\frac{1}{2} \int \frac{d^{3} p}{(2 \pi)^{3}} \phi_{a}(-p) Q^{a b}(p) \phi_{b}(p)
$$

is given by

$$
\left\langle\phi_{a}(p) \phi_{b}(-q)\right\rangle=(2 \pi)^{3} \delta(p-q) Q_{a b}^{-1}(p) .
$$

This rule is correct for both bosons as well as fermions. In the case of the gauge field we have $Q^{3+}=-Q^{+3}=\frac{-i k p_{-}}{4 \pi}$. In the case of the fermionic field we have $Q^{\bar{\psi} \psi}=i p_{\mu} \gamma^{\mu}+M_{b a r e}$ and $Q^{\psi \bar{\psi}}=i p_{\mu}\left(\gamma^{\mu}\right)^{T}+M_{\text {bare }}$. 


\section{A.3 Feynman gauge}

We may add to the action a covariant gauge fixing term of the form

$$
S_{F}=\frac{k}{4 \pi} \int d^{3} x \xi \operatorname{Tr}\left(\partial_{\mu} A^{\mu}\right)^{2} .
$$

The Feynman gauge is obtained in the limit $\xi \rightarrow \infty$, in which case the propagator for $A_{\mu}$ becomes simply

$$
\left\langle A_{\mu}^{a}(p) A_{\nu}^{b}(-q)\right\rangle=-\frac{4 \pi}{k} \delta^{a b} \epsilon_{\mu \nu \rho} \frac{p^{\rho}}{p^{2}}(2 \pi)^{3} \delta^{3}(p-q) .
$$

\section{B Perturbative Analysis of Fermion Self Energy}

In this Appendix we give some details on the perturbative computation of the fermion self-energy in the three different gauge choices defined in A: the Feynman gauge, temporal gauge, and lightcone gauge. The Feynman gauge has the advantage of being Lorentz covariant, but the cubic Chern-Simons interaction makes computations beyond oneloop complicated. The cubic coupling of the gauge field disappears in temporal and light cone gauges. The temporal gauge has the advantage of having a very simple form of the gauge field propagator, which makes explicit perturbative contributions easy. On the other hand, we will see below that it introduces unphysical logarithmic divergences in the fermion self energy at two loops (which should disappear in gauge invariant correlators). The light cone gauge does not suffer from this log divergence problem. While explicit perturbative calculations of correlation functions of gauge invariant operators are sometimes more conveniently done in temporal gauge, the lightcone gauge allowed us to partially solve the planar limit of the Chern-Simons-fermion theory (to all loop order).

\section{B.1 Feynman gauge}

In Feynman gauge, the 1-loop free energy is

$$
\begin{aligned}
& \Sigma(p)=2 \pi \lambda \int \frac{d^{3} q}{(2 \pi)^{3}} \epsilon_{\mu \nu \rho} \frac{q^{\rho}}{q^{2}} \gamma^{\mu} \frac{-i(\not p+\not q)}{(p+q)^{2}} \gamma^{\nu} \\
& =-i 2 \pi \lambda \int \frac{d^{3} q}{(2 \pi)^{3}} \frac{q^{\rho}(p+q)^{\sigma}}{q^{2}(p+q)^{2}} \epsilon_{\mu \nu \rho} \gamma^{\mu} \gamma_{\sigma} \gamma^{\nu} \\
& =-4 \pi \lambda \int \frac{d^{3} q}{(2 \pi)^{3}} \frac{q \cdot(p+q)}{q^{2}(p+q)^{2}}=-2 \pi \lambda \int \frac{d^{3} q}{(2 \pi)^{3}} \frac{(p+q)^{2}+q^{2}-p^{2}}{q^{2}(p+q)^{2}} \\
& =\frac{\pi}{4} \lambda|p|
\end{aligned}
$$


The 1-loop corrected fermion propagator $\left\langle\psi_{i \alpha}(p) \bar{\psi}_{\beta}^{j}(p)\right\rangle$ is then proportional to $\lambda \delta_{i}^{j} \epsilon_{\alpha \beta} /|p|$.

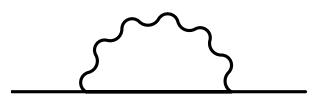

\section{B.2 Temporal gauge}

In the temporal gauge $A_{3}=0$, the one-loop fermion self energy is

$$
\begin{aligned}
& \Sigma(p)=2 \pi \lambda \int \frac{d^{3} \vec{q}}{(2 \pi)^{3}} \epsilon_{i j} \gamma^{i} \frac{p^{3}-q^{3}}{\left(p^{3}-q^{3}\right)^{2}+\epsilon^{2}} \frac{-i \not q}{q^{2}} \gamma^{j} \\
& =-i 2 \pi \lambda \int \frac{d^{3} \vec{q}}{(2 \pi)^{3}} \epsilon_{i j} \gamma^{i} \frac{p^{3}-q^{3}}{\left(p^{3}-q^{3}\right)^{2}+\epsilon^{2}} \frac{q^{3} \gamma^{3}}{q^{2}} \gamma^{j} \\
& =-\frac{8 \pi^{2} \lambda}{(2 \pi)^{3}} \int_{0}^{\infty} d Q Q \int d q^{3} \frac{p^{3}-q^{3}}{\left(p^{3}-q^{3}\right)^{2}+\epsilon^{2}} \frac{q^{3}}{\left(q^{3}\right)^{2}+Q^{2}} \\
& =\lambda \int_{0}^{\infty} d Q \frac{Q^{2}}{\left(p^{3}\right)^{2}+Q^{2}} \\
& =\lambda\left(\Lambda-\frac{\pi}{2}\left|p^{3}\right|\right)
\end{aligned}
$$

After subtracting off the linear divergence by tuning the bare mass of the fermion, we end up with the renormalized 1-loop self energy

$$
\Sigma_{1}(p)=-\frac{\pi}{2} \lambda\left|p^{3}\right| .
$$

We can carry on to the two-loop self energy in temporal gauge, which only receives the contribution from the rainbow diagram. It is given by

$$
\begin{aligned}
& \Sigma_{2}(p)=-2 \pi \lambda \int \frac{d^{3} q}{(2 \pi)^{3}} \frac{p^{3}+q^{3}}{\left(p^{3}+q^{3}\right)^{2}+\epsilon^{2}} \epsilon_{i j} \gamma^{i} \frac{1}{q^{2}} \Sigma_{1}(p) \gamma^{j} \\
& =i \frac{(4 \pi \lambda)^{2}}{4} \gamma^{3} \int \frac{d^{3} q}{(2 \pi)^{3}} \frac{p^{3}+q^{3}}{\left(p^{3}+q^{3}\right)^{2}+\epsilon^{2}} \frac{\left|q^{3}\right|}{q^{2}} \\
& =i \lambda^{2} \gamma^{3} \int_{0}^{\infty} d Q Q \frac{p^{3} \ln \frac{\left(p^{3}\right)^{2}}{Q^{2}}}{\left(p^{3}\right)^{2}+Q^{2}} \\
& =i \lambda^{2} p^{3} \gamma^{3}\left[\frac{\pi^{2}}{12}-\left(\ln \frac{\Lambda}{\left|p^{3}\right|}\right)^{2}\right]
\end{aligned}
$$

We believe that the $\log ^{2}$ divergence is an artifact of the temporal gauge and should drop out in gauge invariant observables. A systematic treatment of regularization in the 
temporal gauge appears to be rather complicated. In the one and two loop corrections to three point functions of gauge invariant operators that we will perform later, such divergences are avoided by special choices of position and polarization configurations of the operator insertions.

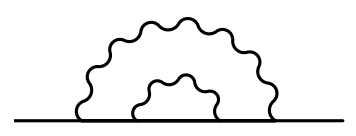

\section{B.3 Light cone gauge}

The 1-loop fermion self energy in the light cone gauge is (note the Gamma/Pauli matrix identities $\gamma^{+} \gamma^{-}=1+\gamma^{3}, \gamma^{-} \gamma^{+}=1-\gamma^{3}$ )

$$
\begin{aligned}
& -4 \pi i \lambda \int \frac{d^{3} q}{(2 \pi)^{3}}\left(\gamma^{3} \frac{-i \not q}{q^{2}+i \epsilon} \gamma^{+}-\gamma^{+} \frac{-i \not q}{q^{2}+i \epsilon} \gamma^{3}\right) \frac{(p-q)^{-}}{(p-q)_{s}^{2}+i \epsilon} \\
& =8 \pi \lambda \int \frac{d^{3} q}{(2 \pi)^{3}} \frac{q^{+}}{q^{2}+i \epsilon} \frac{(p-q)^{-}}{(p-q)_{s}^{2}+i \epsilon}
\end{aligned}
$$

We will now use dimensional reduction to regularize the integral, which will turn out to be convenient at two loops and higher. The integral can be done by separating out two lightcone directions $q^{+}, q^{-}$, and the remaining $1-\epsilon$ dimension. After performing the $1-\epsilon$ dimensional integral, we obtain (constant factors of the form $1+\mathcal{O}(\epsilon)$ are ignored, as there is no logarithmic divergence here)

$$
\begin{aligned}
& 4 \pi \lambda \int \frac{d^{2} q_{s}}{(2 \pi)^{2}} \frac{q^{+}}{\left|q_{s}\right|^{1+\epsilon}} \frac{(p-q)^{-}}{(p-q)_{s}^{2}+i \epsilon} \\
& =-2 \pi \lambda(1+\epsilon) \int_{0}^{1} d x(1-x)^{-\frac{1}{2}+\frac{\epsilon}{2}} \int \frac{d^{2} q_{s}}{(2 \pi)^{2}} \frac{(q+x p)^{+}(q-(1-x) p)^{-}}{\left[q_{s}^{2}+p_{s}^{2} x(1-x)+i \epsilon\right]^{\frac{3}{2}+\epsilon}} \\
& =-\pi \lambda(1+\epsilon) \int_{0}^{1} d x(1-x)^{-\frac{1}{2}+\frac{\epsilon}{2}} \int \frac{d^{2} q_{s}}{(2 \pi)^{2}} \frac{q_{s}^{2}-x(1-x) p_{s}^{2}}{\left[q_{s}^{2}+p_{s}^{2} x(1-x)+i \epsilon\right]^{\frac{3}{2}+\frac{\epsilon}{2}}} \\
& =-\frac{\lambda}{2} \int_{0}^{1} d x(1-x)^{-\frac{1}{2}}\left[-3\left|p_{s}\right| x^{\frac{1}{2}}(1-x)^{\frac{1}{2}}\right] \\
& =\lambda\left|p_{s}\right|
\end{aligned}
$$

So with dimensional reduction, the bare fermion mass is zero, and we find the renormalized 1-loop self energy

$$
\Sigma_{1}(p)=\lambda\left|p_{s}\right| .
$$


It is useful to write the 1-loop correction to the fermion propagator in position space,

$$
-\lambda \int \frac{d^{3} p}{(2 \pi)^{3}} \frac{\left|p_{s}\right|}{p^{2}+i \epsilon} e^{i p \cdot x}=-\frac{\lambda}{4 \pi} \frac{\left|x^{0}\right|}{|x|^{3}}
$$

The only potentially nontrivial planar contribution to the 2-loop fermion self energy comes from the rainbow diagram. It is given by

$$
\begin{aligned}
& -i 4 \pi \lambda^{2} \int \frac{d^{3-\epsilon} q}{(2 \pi)^{3-\epsilon}}\left[\gamma^{3}, \gamma^{+}\right] \frac{\left|q_{s}\right|}{q^{2}+i \epsilon} \frac{(p-q)^{-}}{(p-q)_{s}^{2}+i \epsilon} \\
& \longrightarrow-i 4 \pi \lambda^{2} \gamma^{+} \int \frac{d^{2} q_{s}}{(2 \pi)^{2}} \frac{1}{\left|q_{s}\right|} \frac{(p-q)^{-}}{(p-q)_{s}^{2}+i \epsilon}=-i \lambda^{2} p^{-} \gamma^{+},
\end{aligned}
$$

where the integral in the last step is performed as in section 2.1.1. From this we find the two loop contribution to the fermion self energy,

$$
\Sigma_{2}(p)=-i \lambda^{2} p_{+} \gamma^{+} .
$$

As shown in section 2, the planar fermion self energy is in fact two-loop exact in the dimensional reduction scheme.

\section{Lorentz invariance of the Wilson line at one loop}

30

The exact fermion propagator computed earlier in this paper in lightcone gauge is not Lorentz invariant. As the fermion propagator by is not gauge invariant, it is not physically observable. In this section we will compute a gauge invariant physical observable that is closely related to the fermion propagator, and demonstrate that this observable is Lorentz invariant (at one loop), and moreover takes the same value in lightcone gauge as in Feynman gauge. Our results may be taken as evidence that the lightcone gauge employed in this paper defines a Lorentz invariant theory, atleast at first order in $\lambda$.

Consider the Wilson line

$$
W=\frac{1}{N}\left\langle 0\left|\left[P e^{i \int_{x_{i}}^{x_{f}} \vec{A} \cdot d \vec{l}}\right]_{m}^{n} \psi\left(x_{i}\right)_{n} \bar{\psi}\left(x_{f}\right)^{m}\right| 0\right\rangle
$$

where $m, n$ are colour indices, we do not indicate the spinor indices of $\psi\left(x_{i}\right), \bar{\psi}\left(x_{f}\right)$ explicitly (the Wilson line is a matrix in spinor space, in the same way that the propagator of the previous section is). The expectation value of the Wilson line operator described above is Lorentz invariant if and only if it takes the form

$$
\left(x_{f}-x_{i}\right)_{\mu} \gamma^{\mu} A\left(\left|x_{f}-x_{i}\right|\right)+I B\left(\left|x_{f}-x_{i}\right|\right) .
$$

\footnotetext{
${ }^{30}$ This appendix was worked out in collaboration with Sayantani Bhattacharyya.
} 
In this subsection we will demonstrate, at the one loop level, that the expectation value of the Wilson loop in lightcone gauge really does take this form (at one loop we find that $A$ vanishes while $B$ is nonzero).

At leading order, the Wilson line defined above is simply given by

$$
\int \frac{d^{3} p}{(2 \pi)^{3}} e^{i\left(x_{i}-x_{f}\right) \cdot p} \frac{1}{i p_{\mu} \gamma^{\mu}}
$$

and is manifestly rotationally invariant.

At next to leading order (or leading non-trivial order in $\lambda$ ) the Wilson loop receives contributions from two graphs. The first arises from the leading self energy correction to the $\psi \bar{\psi}$ propagator. In the lightcone gauge it is given by

$$
W_{1}=(4 \pi \lambda) \int \frac{d^{3} q}{(2 \pi)^{3}} \frac{d^{3} p}{(2 \pi)^{3}} \frac{e^{i p \cdot\left(x_{i}-x_{f}\right)}}{p^{2} q^{2}} \frac{q^{+}}{(p-q)^{+}} .
$$

As we have seen above this diagram is easily evaluated, however we will find it useful to leave the integral unevaluated at this stage.

The second graph has one insertion of the "Wilson line factor" $\int_{x_{i}}^{x^{f}} \vec{A} \cdot d \vec{l}$ along the $\hat{y}$ direction (direction from $x_{i}$ to $x_{f}$ ) ; this graph evaluates to

$$
W_{2}=\frac{i N}{2} \int \frac{d^{3} p}{(2 \pi)^{3}} \frac{d^{3} p^{\prime}}{(2 \pi)^{3}}\left(e^{i p \cdot\left(x_{i}-x_{f}\right)}-e^{i p^{\prime} \cdot\left(x_{i}-x_{f}\right)}\right) \frac{p^{\rho} \gamma_{\rho} \gamma^{\mu} p^{\prime \sigma} \gamma_{\sigma}}{p^{2} p^{\prime 2}\left(\left(p-p^{\prime}\right) \cdot \hat{y}\right)} G_{\mu \hat{y}}\left(p-p^{\prime}\right)
$$

(this result is correct both in lightcone gauge and in the Lorentz invariant gauge we will employ below). In lightcone gauge, it is possible to show that this diagram evaluates to

$$
W_{2}=-4 \pi \lambda \int \frac{d^{3} q}{(2 \pi)^{3}} \frac{d^{3} p}{(2 \pi)^{3}} \frac{e^{i p \cdot\left(x_{i}-x_{f}\right)}}{p^{2} q^{2}}\left(\frac{q^{+}}{(p-q)^{+}}-\frac{q \cdot \hat{y}}{(p-q) \cdot \hat{y}}\right)
$$

In order to obtain (C.4 we have assumed that our regulator respect the 3 to -3 flip symmetry as well as rotational invariance in the 12 plane. These properties are both obviously true of the dimensional regulator employed in this paper.

It is obvious that neither $W_{1}$ nor $W_{2}$ is rotationally invariant by itself. However

$$
W=W_{1}+W_{2}=4 \pi \lambda \int \frac{d^{3} q}{(2 \pi)^{3}} \frac{d^{3} p}{(2 \pi)^{3}} \frac{e^{i p \cdot\left(x_{i}-x_{f}\right)}}{p^{2} q^{2}} \frac{q \cdot \hat{y}}{(p-q) \cdot \hat{y}}
$$

Note that a rotation of $x_{f}-x_{i}$ can be undone by a rotation of the dummy variables $p$ and $q$ establishing that the Wilson line depends only on $\left|x_{f}-x_{i}\right|$, establishing rotational invariance in any regularization scheme - like the dimensional reduction scheme employed in this paper - that respects rotational invariance. 
It is interesting that the calculation of the Wilson loop proceeds along very similar lines and yields exactly the same answer in Feynman gauge. In this gauge, once again, we receive contributions from two graphs. The self energy graph gives

$$
W_{1}^{\prime}=(4 \pi \lambda) \int \frac{d^{3} q}{(2 \pi)^{3}} \frac{d^{3} p}{(2 \pi)^{3}} \frac{e^{i p \cdot\left(x_{i}-x_{f}\right)}}{p^{2} q^{2}} \frac{q \cdot(p-q)}{(p-q)^{2}}
$$

The second graph is given by (C.3) in which we must use the gauge propagator appropriate to the Feynman gauge, and evaluates to

$$
W_{2}^{\prime}=-4 \pi \lambda \int \frac{d^{3} q}{(2 \pi)^{3}} \frac{d^{3} p}{(2 \pi)^{3}} \frac{e^{i p \cdot\left(x_{i}-x_{f}\right)}}{p^{2} q^{2}}\left(\frac{q \cdot(p-q)}{(p-q)^{2}}-\frac{q \cdot \hat{y}}{(p-q) \cdot \hat{y}}\right)
$$

Clearly $W_{1}^{\prime}+W_{2}^{\prime}=W_{1}+W_{2}=W$ where $W$ is listed in C.5.

\section{Unitary Representations of the $d=3$ Conformal Group}

Unitary representations of the conformal group are labeled by the spin $s$ and a scaling dimension $\Delta$ of their primary states. When $s \geq 1$ these labels are subject to the inequality $\Delta \geq s+1$. In this case representations that saturate the inequality are short; the null states in this representation fall into a (long) representation with $\Delta=s+2$ and $\operatorname{spin}=s-1$.

In the special case $s=\frac{1}{2}$ it turns out that $\Delta \geq 1$. The representation with $\Delta=1$ is short and its null states fall into a (long) representation with $\Delta=2$ and spin $=\frac{1}{2}$. The later is the representation of a free fermionic field, and the character (3.1) $F_{F}(x, \mu)$ of this field is given by

$$
F_{F}(x, \mu)=\frac{x\left(\mu^{\frac{1}{2}}+\mu^{-\frac{1}{2}}\right)}{(1-\mu x)\left(1-\mu^{-1} x\right)}
$$

Finally, when $s=0$ we have $\Delta=0$ or $\Delta \geq \frac{1}{2}$. The representation with $\Delta=0$ has a single state. The representation with $\Delta=\frac{1}{2}$ has null states in a representation with $\Delta=\frac{5}{2}$ and $s=0$. The states of a free scalar field fall into this representation; the character $F_{S}(x, \mu)$ of this representation is given by

$$
F_{S}(x, \mu)=x^{\frac{1}{2}} \frac{(1+x)}{(1-\mu x)\left(1-\mu^{-1} x\right)}
$$

We now present character formulae for all unitary representations of the $3 \mathrm{~d}$ conformal algebra. Let us define

$$
\chi_{s}(\mu)=\sum_{j=-s}^{s} \mu^{j}
$$


$\chi_{s}(\mu)$ is, of course, the $S U(2)$ character at spin $s$ (here $s$ is a positive integer or half integer). Let us also define

$$
G_{\Delta, s}=\frac{x^{\delta} \chi_{s}(\mu)}{(1-x)(1-\mu x)\left(1-\mu^{-1} x\right)}
$$

$G_{\Delta, s}$ is the partition function over states that are obtained by acting on an $S U(2)$ primary of spin $s$ with an arbitrary number of derivatives, and so yields the character of any long representation of the conformal algebra (i.e. any representation with $\Delta>s+1$ for $s \geq 1$ or $\Delta>1$ for $s=\frac{1}{2}$ or $\Delta>\frac{1}{2}$ for $s=0$ ).

Characters $\chi(x, \mu)$ of short representations of the conformal algebra are obtained by evaluating the character of a hypothetical long representation of that algebra and then subtracting out the character of its null states. It follows that, for $s \geq 1$

$$
\chi_{s+1, s}(x, \mu)=G_{s+1, s}(x, \mu)-G_{s+2, s-1}(x, \mu)
$$

For $s=\frac{1}{2}$

$$
\chi_{1, \frac{1}{2}}(x, \mu)=G_{1, \frac{1}{2}}(x, \mu)-G_{2, \frac{1}{2}}(x, \mu)=F_{F}(x, \mu)
$$

while for $s=0$

$$
\chi_{\frac{1}{2}, 0}(x, \mu)=G_{\frac{1}{2}, 0}(x, \mu)-G_{\frac{5}{2}, 0}(x, \mu)=F_{S}(x, \mu)
$$

\section{E Primary Operators in Free and Interacting Fermion Theories}

In this appendix we present the details of some slightly tedious computations involving free and interacting fermions.

\section{E.1 The generating function of conserved currents for free fermions}

As we have explained in section 3 above, the single-trace primary operators of the free fermion theory satisfy the condition that their scaling dimension, $\epsilon$ and spin $s$ satisfy the relation $\epsilon=s+1$, with the exception of $\bar{\psi} \psi$, which has scaling dimension 2 and spin 0 . Here, we will determine explicit expressions for the corresponding primary operators.

How can we produce an operator of spin $s$ and dimension $s+1$ in a theory of free fermions in $d=3$ ? We are interested in operators built out of fermion bilinears (with colour indices contracted). As $\psi$ and $\bar{\psi}$ each have unit scaling dimension, the operator 
of interest must contain exactly $s-1$ derivatives. As $s-1$ derivatives can give rise to at most $s-1$ free traceless vector indices the remaining index (to make our operator spin $s$ ) must come from a $\gamma$ matrix. ${ }^{31}$ Consequently if we define the generating function $F$ such that

$$
\mathcal{O}(x ; \epsilon)=\bar{\psi} F\left(\vec{\gamma}, \overrightarrow{\partial_{\mu}}, \overleftarrow{\partial_{\mu}}, \vec{\epsilon}\right) \psi=\sum J_{\mu_{1} \mu_{2} \ldots \mu_{s}}^{(s)} \epsilon^{\mu_{1}} \ldots \epsilon^{\mu_{s}}
$$

then $F$ must take the form

$$
F=\vec{\gamma} \cdot \vec{\epsilon} f\left(\overrightarrow{\partial_{\mu}}, \overleftarrow{\partial_{\mu}}, \vec{\epsilon}\right)
$$

Following 31 we denote the arguments of $f$ as vectors $u$ and $v$, so that $\vec{u}=\overleftarrow{\partial}$ and $\vec{v}=\vec{\partial}$. The fermion equation of motion gives

$$
\vec{u} \cdot \vec{\gamma}=\vec{v} \cdot \gamma=u^{2}=v^{2}=0
$$

It is convenient to change variables to $\vec{y} \equiv \vec{u}-\vec{v}$ and $\vec{z} \equiv \vec{u}+\vec{v}$. Then we have:

$$
\vec{z} \cdot \vec{\gamma}=\vec{y} \cdot \vec{\gamma}=\vec{y} \cdot \vec{z}=0, \quad-\vec{y}^{2}=\vec{z}^{2}=2 \vec{u} \cdot \vec{v} \neq 0
$$

Terms in $f$ will be of the form:

$$
f=A+B \vec{\epsilon} \cdot \vec{y}+C \vec{\epsilon} \cdot \vec{z}+D \vec{u} \cdot \vec{v} \vec{\epsilon} \cdot \vec{\epsilon}+\ldots
$$

where each coefficient is a number.

If we define $w \equiv(\vec{u} \cdot \vec{v}) \vec{\epsilon} \cdot \vec{\epsilon}, z \equiv \vec{\epsilon} \cdot \vec{z}$, and $y=\vec{\epsilon} \cdot \vec{y}$, then $f$ can be thought of as a function of three variables $f(z, y, w)$.

The condition that each current is conserved can be expressed as:

$$
\left(\overleftarrow{\partial_{\mu}}+\overrightarrow{\partial_{\mu}}\right) \frac{\partial}{\partial \epsilon_{\mu}} F=0
$$

which translates into the following condition on $f$ :

$$
z \partial_{w} f+\partial_{z} f=0
$$

The condition that each current is traceless can be expressed as:

$$
\frac{\partial}{\partial \epsilon^{\mu}} \frac{\partial}{\partial \epsilon_{\mu}} F=0
$$

\footnotetext{
${ }^{31} \mathrm{As} \gamma_{(\mu} \gamma_{\nu)}=\eta_{\mu \nu}$ we cannot have more than one of the current indices come from a $\gamma$ matrix. Also, the equations of motion tell us that a $\gamma$ matrix contracted with a derivative vanishes in its action on a fermion. The contraction of a derivative with a $\gamma$ matrix, sandwiched by other $\gamma$ matrices, can also be reduced to a form with a single $\gamma$ matrix and derivatives using the equations of motion, (e.g. $\gamma_{(\mu} \gamma_{\rho} \partial^{\rho} \gamma_{\nu)}=-\gamma_{(\mu} \partial^{\rho} \gamma_{\nu)} \gamma_{\rho}+\gamma_{(\mu} \partial^{\rho} \eta_{\nu) \rho}$ where the first term on the RHS vanishes by the equations of motion and the last term has a single $\gamma$ matrix).
} 
which translates into:

$$
\left(5 \partial_{w}+2 w \partial_{w}^{2}-\partial_{y}^{2}+\partial_{z}^{2}+2 z \partial_{z} \partial_{w}+2 y \partial_{y} \partial_{w}\right) f=0
$$

The solution to E.7) is $f(w, y, z)=g\left(y, w-\frac{z^{2}}{2}\right)$. If we define $t=w-\frac{z^{2}}{2}$, the equation for $g(y, t)$ is

$$
\left(4 \partial_{t}+2 t \partial_{t}^{2}-\partial_{y}^{2}+2 y \partial_{y} \partial_{t}\right) g(y, t)=0
$$

The general solution satisfying $g=1$ at $t=y=0$ is:

$$
g=e^{2 k y} \frac{\sinh 2 k \sqrt{2 t+y^{2}}}{2 k \sqrt{2 t+y^{2}}}
$$

where $k$ is any constant, which we take to be $1 / 2$.

The final form for $f$ is thus

$$
f(\vec{u}, \vec{v}, \vec{\epsilon})=\frac{\exp (\vec{u} \cdot \vec{\epsilon}-\vec{v} \cdot \vec{\epsilon}) \sinh \sqrt{2 \vec{u} \cdot \vec{v} \vec{\epsilon} \cdot \vec{\epsilon}-4 \vec{u} \cdot \vec{\epsilon} \vec{v} \cdot \vec{\epsilon}}}{\sqrt{2 \vec{u} \cdot \vec{v} \vec{\epsilon} \cdot \vec{\epsilon}-4 \vec{u} \cdot \vec{\epsilon} \vec{v} \cdot \vec{\epsilon}}}
$$

Expanding the above expression in a power series around $\vec{\epsilon}$, we obtain:

$$
\begin{aligned}
f= & 1+\epsilon(u-v)+\frac{1}{6} \epsilon^{2}\left(3 u^{2}-10 u v+3 v^{2}+2 w\right) \\
& +\epsilon^{3}\left(\frac{u^{3}}{6}-\frac{7 u^{2} v}{6}+\frac{7 u v^{2}}{6}+\frac{u w}{3}-\frac{v^{3}}{6}-\frac{v w}{3}\right) \\
& +\frac{1}{120} \epsilon^{4}\left(10(u-v)^{2}(2 w-4 u v)+(4 u v-2 w)^{2}+5(u-v)^{4}\right)
\end{aligned}
$$

(above, $w=\vec{u} \cdot \vec{v}, u=\vec{u} \cdot \vec{\epsilon}, v=\vec{v} \cdot \vec{\epsilon}$.) which yields the currents reported in (3.9).

\section{E.2 Two-point functions of primary operators in the free the- ory}

In this subsection we explicitly compute the two-point function of conserved currents

$$
\left\langle\mathcal{O}\left(\vec{x} ; \vec{\epsilon}_{1}\right) \mathcal{O}\left(0 ; \vec{\epsilon}_{2}\right)\right\rangle
$$

determined in the previous subsection, and demonstrate that the two point functions of currents of different spin are orthonormal. We take the two-point function of the basic fermionic fields to be given by

$$
\langle\psi(x) \bar{\psi}(0)\rangle=C_{\psi \psi} \frac{\gamma^{\mu} x_{\mu}}{x^{3}} .
$$




\section{E.2.1 Simple examples}

To set up notation and get intuition we first work out some simple examples.

As a first example, consider the two-point function of two scalar currents:

$$
\langle\bar{\psi}(x) \psi(x) \bar{\psi}(0) \psi(0)\rangle
$$

Using Wick's theorem we rewrite this as:

$$
\operatorname{Tr}\langle-\psi(0) \bar{\psi}(x)\rangle\langle\psi(x) \bar{\psi}(0)\rangle
$$

where the trace is over gamma matrix indices. We then compute it to be:

$$
\begin{aligned}
& C_{\psi \psi}^{2} \operatorname{Tr} \frac{\gamma^{\mu} x_{\mu}}{x^{3}} \frac{\gamma^{\nu} x_{\nu}}{x^{3}}=C_{\psi \psi}^{2} \frac{x_{\nu} x_{\mu}}{x^{6}} \operatorname{Tr} \gamma^{\mu} \gamma^{\nu} \\
& =C_{\psi \psi}^{2} \frac{x_{\nu} x_{\mu}}{x^{6}} 2 \eta^{\mu \nu}=C_{\psi \psi}^{2} \frac{2}{x^{4}} .
\end{aligned}
$$

To evaluate more complicated two-point functions, we make use of the identity

$$
\operatorname{Tr} \gamma^{\mu} \gamma^{\rho} \gamma^{\nu} \gamma^{\sigma}=2\left(\eta^{\rho \nu} \eta^{\sigma \mu}+\eta^{\rho \mu} \eta^{\sigma \nu}-\eta^{\rho \sigma} \eta^{\mu \nu}\right)
$$

Note that (E.17) is symmetric under interchange of $\rho$ and $\sigma$.

We next consider the two-point function of two spin-1 currents:

$$
\left\langle\bar{\psi}(x) \gamma^{\nu} \psi(x) \bar{\psi}(y) \gamma^{\mu} \psi(y)\right\rangle
$$

We have:

$$
\begin{aligned}
\left\langle\bar{\psi}(x) \gamma^{\mu} \psi(x) \bar{\psi}(y) \gamma^{\nu} \psi(y)\right\rangle & =\operatorname{Tr}\langle-\psi(y) \bar{\psi}(x)\rangle \gamma^{\mu}\langle\psi(x) \bar{\psi}(y)\rangle \gamma^{\nu} \\
& =C_{\psi \psi}^{2} \frac{x_{\rho}}{x^{3}} \frac{x_{\sigma}}{x^{3}} \operatorname{Tr} \gamma^{\rho} \gamma^{\nu} \gamma^{\sigma} \gamma^{\mu} \\
& =C_{\psi \psi}^{2} \frac{x_{\rho}}{x^{3}} \frac{x_{\sigma}}{x^{3}} 2\left(\eta^{\rho \nu} \eta^{\sigma \mu}+\eta^{\rho \mu} \eta^{\sigma \nu}-\eta^{\rho \sigma} \eta^{\mu \nu}\right) \\
& =2 C_{\psi \psi}^{2}\left(\frac{2 x^{\mu} x^{\nu}}{x^{6}}-\frac{\eta^{\mu \nu}}{x^{4}}\right)
\end{aligned}
$$

As above, we will often set $y=0$ in the last line.

\section{E.2.2 Results for all spins}

Let $\varepsilon$ be a null polarization vector. The two point function of the generating operator $\mathcal{O}(x ; \varepsilon)=\sum J_{\mu_{1} \cdots \mu_{s}}^{(s)} \varepsilon^{\mu_{1}} \cdots \varepsilon^{\mu_{s}}$ is evaluated in the theory of $N$ free complex fermions to be

$$
\langle\mathcal{O}(x ; \varepsilon) \mathcal{O}(0 ; \varepsilon)\rangle=\frac{N}{32 \pi^{2} x^{2}}\left\{\left[1-\left(\frac{4 \varepsilon \cdot x}{x^{2}}\right)^{2}\right]^{-\frac{1}{2}}-1\right\} .
$$


Expanding this in $\varepsilon$, we have

$$
\left\langle J^{(s)}(x ; \varepsilon) J^{(s)}(0 ; \varepsilon)\right\rangle=\frac{N}{32 \pi^{\frac{5}{2}}} \frac{2^{4 s} \Gamma\left(s+\frac{1}{2}\right)}{s !} \frac{(\varepsilon \cdot x)^{2 s}}{\left(x^{2}\right)^{2 s+1}},
$$

where $J^{(s)}(x ; \varepsilon)$ is the spin-s part of $\mathcal{O}(x ; \varepsilon)$. The spin 0 case is special, where we have $\left\langle J^{(0)}(x) J^{(0)}(0)\right\rangle=\frac{N}{8 \pi^{2}}|x|^{-4}$.

We have defined the set of currents $j^{(s)}$ with a different normalization convention, namely normalizing the norm of the corresponding state in radial quantization. The relative normalization between $J^{(s)}$ and $j^{(s)}$ can be determined as follows. If we define $j^{(s)}(x ; \varepsilon)=j_{\mu_{1} \cdots \mu_{s}}^{(s)}(x) \varepsilon^{\mu_{1}} \cdots \varepsilon^{\mu_{s}}$, then

$$
\left\langle j^{(s)}(x ; \varepsilon) j^{(s)}(0 ; \varepsilon)\right\rangle=2^{s} \frac{(\varepsilon \cdot x)^{2 s}}{\left(x^{2}\right)^{2 s+1+\delta_{s}}},
$$

for $s>0$. In the spin 0 case, we have $\left\langle j^{(0)}(x) j^{(0)}(0)\right\rangle=|x|^{-4}$. From this we deduce

$$
J_{\underline{\mu}}^{(s)}(x)=a_{s} j_{\underline{\mu}}^{(s)}(x), \quad a_{s}=\left[\frac{N}{32 \pi^{\frac{5}{2}}} \frac{2^{3 s} \Gamma\left(s+\frac{1}{2}\right)}{s !}\right]^{\frac{1}{2}},
$$

for $s>0$. In the spin 0 case, $a_{0}=\frac{\sqrt{2 N}}{4 \pi}$. In the interacting Chern-Simons-fermion theory, $a_{s}$ receives quantum corrections.

\section{E.3 Explicit computation of the divergence of $J^{(3)}$}

In carrying out all our manipulations below, we use the fermion equation of motion

$$
D_{\mu} \gamma^{\mu} \psi=D_{\mu} \bar{\psi} \gamma^{\mu}=0
$$

Some useful identities are:

$$
\begin{aligned}
& \gamma^{\mu} D_{\mu} D_{\nu} \psi=\gamma^{\mu}\left(D_{\nu} D_{\mu}-i F_{\mu \nu}\right) \psi=-i \gamma^{\mu} F_{\mu \nu} \psi \\
& D_{\mu} D_{\nu} \bar{\psi} \gamma^{\mu}=\left(D_{\nu} D_{\mu}+i F_{\mu \nu}\right) \bar{\psi} \gamma^{\mu}=i \bar{\psi} \gamma^{\mu} F_{\mu \nu} \\
& \gamma^{\mu} D_{\mu} \gamma^{\nu} D_{\nu} \psi=0 \\
& \left(\eta^{\mu \nu}+\gamma^{\mu \nu}\right) D_{\mu} D_{\nu} \psi=0 \\
& D_{\mu} D^{\mu} \psi=\frac{i}{2} \gamma^{\mu \nu} F_{\mu \nu} \psi \\
& D_{\mu} D_{\nu} \bar{\psi} \gamma^{\nu} \gamma^{\mu}=0 \\
& D_{\mu} D^{\mu} \bar{\psi}=\frac{i}{2} \bar{\psi} \gamma^{\mu \nu} F_{\mu \nu}
\end{aligned}
$$


Note our convention is such that $\left[\vec{D}_{\mu}, \vec{D}_{\nu}\right] \psi=-i F_{\mu \nu} \psi$. We now use the equation of motion for $F_{\mu \nu}=F_{\mu \nu}^{a} T^{a}$, namely

$$
\epsilon^{\mu \nu \rho} F_{\nu \rho}=\frac{4 \pi}{k} J^{\mu}, \quad \text { or } \quad F_{\mu \nu}^{a}=\frac{2 \pi}{k} \epsilon_{\mu \nu \rho} J^{a \rho},
$$

where $J_{\mu}=J_{\mu}^{a} T^{a}, J_{\mu}^{a}=\bar{\psi} \gamma_{\mu} T^{a} \psi$. It is also useful to have

$$
\begin{aligned}
J_{\rho}^{a} T^{a} \psi & =\left(\bar{\psi} \gamma_{\rho} T^{a} \psi\right) T^{a} \psi \\
& =-\frac{1}{4}\left(\gamma_{\rho} \psi(\bar{\psi} \psi)+\gamma^{\mu} \gamma^{\rho} \psi\left(\bar{\psi} \gamma_{\mu} \psi\right)\right) \\
& =-\frac{1}{4}\left(\gamma_{\rho} \psi J^{(0)}+\gamma^{\mu} \gamma_{\rho} \psi J_{\mu}^{(1)}\right)
\end{aligned}
$$

and

$$
\bar{\psi} J_{\rho}^{a} T^{a}=-\frac{1}{4}\left(J^{(0)} \bar{\psi} \gamma_{\rho}+J_{\mu}^{(1)} \bar{\psi} \gamma_{\rho} \gamma^{\mu}\right) .
$$

To derive these relations, we used $\left(T^{a}\right)_{j}^{i}\left(T^{a}\right)_{m}^{l}=\frac{1}{2} \delta_{m}^{i} \delta_{j}^{l}$ and the $3 \mathrm{~d}$ Fierz identity

$$
\chi \bar{\lambda}=-\frac{1}{2} \bar{\lambda} \chi-\frac{1}{2} \bar{\lambda} \gamma_{\mu} \chi \gamma^{\mu}
$$

We can now proceed to explicitly compute $\partial^{\mu} J_{\mu \nu_{1} \nu_{2}}^{(3)}$. First, consider the current $\hat{J}^{(3)}$ which is not traceless,

$$
\hat{J}_{\mu_{1} \mu_{2} \mu_{3}}^{(3)}=\frac{1}{6} \bar{\psi} \gamma_{\mu_{1}}\left(3 \overleftarrow{D_{\mu_{2}}} \overleftarrow{D_{\mu_{3}}}-10 \overleftarrow{D_{\mu_{2}}} \overrightarrow{D_{\mu_{3}}}+3 \overrightarrow{D_{\mu_{2}}} \overrightarrow{D_{\mu_{3}}}+2\left(\overleftarrow{D_{\sigma}} \overrightarrow{D^{\sigma}}\right) \eta_{\mu_{2} \mu_{3}}\right) \psi
$$

Using the identities above we find that (before subtracting the trace) the divergence is given by:

$$
\begin{aligned}
6 \partial^{\mu} \hat{J}_{\mu \nu_{1} \nu_{2}}^{(3)}= & -i \bar{\psi} \gamma^{\mu}\left(16 \overleftarrow{D}_{\nu_{1}} F_{\nu_{2} \mu}+16 F_{\mu \nu_{1}} \vec{D}_{\nu_{2}}+2 \eta_{\nu_{1} \nu_{2}}\left(\overleftarrow{D}_{\lambda} F_{\mu \lambda}+F_{\lambda \mu} \vec{D}_{\lambda}\right)\right) \psi \\
& -i \bar{\psi} \gamma^{\nu_{1}}\left(16\left(\overleftarrow{D}^{\mu} F_{\nu_{2} \mu}+F_{\mu \nu_{2}} \vec{D}^{\mu}\right)\right) \psi \\
& +\bar{\psi} \gamma^{\nu_{1}}\left(6\left(\overleftarrow{D}^{2} \overleftarrow{D}_{\nu_{2}}+\vec{D}_{\nu_{2}} \vec{D}^{2}\right)-10\left(\overleftarrow{D}^{2} \vec{D}_{\nu_{2}}+\overleftarrow{D}_{\nu_{2}} \vec{D}^{2}\right)\right) \psi
\end{aligned}
$$

We now substitute for $D^{2}$ and further simplify:

$$
\begin{aligned}
6 \partial^{\mu} & \hat{J}_{\mu \nu_{1} \nu_{2}}^{(3)}=-i \bar{\psi} \gamma^{\mu}\left(32 \overleftarrow{D}_{\nu_{1}} F_{\nu_{2} \mu}+32 F_{\mu \nu_{1}} \vec{D}_{\nu_{2}}+2 \eta_{\nu_{1} \nu_{2}}\left(\overleftarrow{D}_{\lambda} F_{\mu \lambda}+F_{\lambda \mu} \vec{D}_{\lambda}\right)\right) \psi \\
& -i \bar{\psi} \gamma^{\nu_{1}}\left(16\left(\overleftarrow{D}^{\mu} F_{\nu_{2} \mu}+F_{\mu \nu_{2}} \vec{D}^{\mu}\right)\right) \psi-i \bar{\psi}\left(2 \overleftarrow{D}_{\nu_{1}} \tilde{F}_{\nu_{2}}+2 \tilde{F}_{\nu_{1}} \vec{D}_{\nu_{2}}-6\left(D_{\nu_{1}} \tilde{F}_{\nu_{2}}\right)\right) \psi
\end{aligned}
$$

where $\tilde{F}_{\mu}=\epsilon_{\mu \nu \rho} F^{\nu \rho}$. 
Now substituting in $F$ and using Fierz identity, we have

$$
\begin{aligned}
& -32 i \bar{\psi} \gamma^{\mu}\left(\overleftarrow{D}_{\nu_{1}} F_{\nu_{2} \mu}+F_{\mu \nu_{1}} \vec{D}_{\nu_{2}}\right) \psi=\frac{32 \pi}{k}\left[\partial_{\nu_{1}}(\bar{\psi} \psi) \bar{\psi} \gamma_{\nu_{2}} \psi-\partial_{\nu_{1}}\left(\bar{\psi} \gamma_{\nu_{2}} \psi\right) \bar{\psi} \psi\right] \\
& -2 i \eta_{\nu_{1} \nu_{2}} \bar{\psi} \gamma^{\mu}\left(\overleftarrow{D}_{\lambda} F_{\mu \lambda}+F_{\lambda \mu} \vec{D}_{\lambda}\right) \psi=-\frac{2 \pi}{k} \eta_{\nu_{1} \nu_{2}} \partial_{\mu}(\bar{\psi} \psi) \bar{\psi} \gamma^{\mu} \psi \\
& -i \bar{\psi} \gamma^{\nu_{1}}\left(16\left(\overleftarrow{D}^{\mu} F_{\nu_{2} \mu}+F_{\mu \nu_{2}} \vec{D}^{\mu}\right)\right) \psi=\frac{8 \pi}{k}\left[-\eta_{\nu_{1} \nu_{2}} \partial_{\mu}(\bar{\psi} \psi) \bar{\psi} \gamma^{\mu} \psi+2 \partial_{\nu_{1}}(\bar{\psi} \psi) \bar{\psi} \gamma_{\nu_{2}} \psi\right. \\
& \left.\quad-\partial_{\nu_{1}}\left(\bar{\psi} \gamma_{\nu_{2}} \psi\right) \bar{\psi} \psi+\epsilon_{\nu_{2} \lambda \mu}\left(\bar{\psi} \overleftrightarrow{D}^{\mu} \gamma_{\nu_{1}} \psi\right)\left(\bar{\psi} \gamma^{\lambda} \psi\right)\right] \\
& -i \bar{\psi}\left(2 \overleftarrow{D}_{\nu_{1}} \tilde{F}_{\nu_{2}}+2 \tilde{F}_{\nu_{1}} \vec{D}_{\nu_{2}}\right) \psi=-\frac{2 \pi}{k}\left[\partial_{\nu_{1}}\left(\bar{\psi} \gamma_{\nu_{2}} \psi\right) \bar{\psi} \psi+\left(\bar{\psi} \gamma_{\nu_{2}} \psi\right) \partial_{\nu_{1}}(\bar{\psi} \psi)+\epsilon_{\nu_{2} \lambda \mu}\left(\bar{\psi}_{\nu_{\nu_{1}}} \gamma^{\mu} \psi\right)\left(\bar{\psi} \gamma^{\lambda} \psi\right)\right] \\
& -6 i \bar{\psi}\left(D_{\nu_{1}} \tilde{F}_{\nu_{2}}\right) \psi=\frac{6 \pi}{k}\left[\left(\bar{\psi} \gamma_{\nu_{2}} \psi\right) \partial_{\nu_{1}}(\bar{\psi} \psi)+\partial_{\nu_{1}}\left(\bar{\psi} \gamma_{\nu_{2}} \psi\right) \bar{\psi} \psi-\epsilon_{\nu_{2} \lambda \mu}\left(\bar{\psi} \overleftrightarrow{D}_{\nu_{1}} \gamma^{\mu} \psi\right)\left(\bar{\psi} \gamma^{\lambda} \psi\right)\right]
\end{aligned}
$$

We now use the identity

$$
\epsilon_{\nu_{2} \lambda \mu} \bar{\psi}\left(-\overleftrightarrow{D}_{\nu_{1}} \gamma^{\mu}+\overleftrightarrow{D}^{\mu} \gamma_{\nu_{1}}\right) \psi\left(\bar{\psi} \gamma^{\lambda} \psi\right)=-\eta_{\nu_{1} \nu_{2}} \partial^{\lambda}(\bar{\psi} \psi)\left(\bar{\psi} \gamma_{\lambda} \psi\right)+\partial_{\nu_{1}}(\bar{\psi} \psi) \bar{\psi} \gamma_{\nu_{2}} \psi
$$

to obtain the following total for the above sum:

$$
6 \partial^{\mu} \hat{J}_{\mu \nu_{1} \nu_{2}}^{(3)}=\frac{2 \pi}{k}\left[-9 \eta_{\nu_{1} \nu_{2}} \partial_{\mu}(\bar{\psi} \psi)\left(\bar{\psi} \gamma^{\mu} \psi\right)+30 \partial_{\nu_{1}}(\bar{\psi} \psi) \bar{\psi} \gamma_{\nu_{2}} \psi-18 \partial_{\nu_{1}}\left(\bar{\psi} \gamma_{\nu_{2}} \psi\right) \bar{\psi} \psi\right]
$$

Subtracting the trace we obtain:

$$
\partial^{\mu} J_{\mu \nu_{1} \nu_{2}}^{(3)}=\partial^{\mu} \hat{J}_{\mu \nu_{1} \nu_{2}}^{(3)}-\frac{\pi}{5 k}\left[\eta_{\nu_{1} \nu_{2}}\left(\bar{\psi} \gamma^{\mu} \psi\right) \partial_{\mu}(\bar{\psi} \psi)+2 \partial_{\nu_{1}}\left(\bar{\psi} \gamma_{\nu_{2}} \psi\right) \bar{\psi} \psi+2\left(\bar{\psi} \gamma_{\nu_{2}} \psi\right) \partial_{\nu_{1}}(\bar{\psi} \psi)\right]
$$

The indices on the RHS are understood to be symmetrized.

\section{E.4 Higher spin examples}

While we do not have a general explicit formula expressing the divergence of the current in terms of double and triple trace operators, a straightforward but tedious computation was carried out using Mathematica to determine the double trace terms. We find that $\partial^{\mu} J_{\mu \mu_{1} \cdots \mu_{s-1}}^{(s)}$ involves the product of two currents of spins $s_{1}$ and $s_{2}$, with $s_{1}+s_{2} \equiv$ $s(\bmod 2)$ and $s_{1}+s_{2} \leq s-2$. It is convenient to write the results in spinorial notation. For instance, the anomalous current conservation relation for $J^{(3)}$ takes the form (up to an overall normalization factor)

$$
\partial^{\mu} J_{\mu \alpha_{1} \cdots \alpha_{4}}^{(3)} \sim \frac{1}{k}\left[3 \partial_{\left(\alpha_{1} \alpha_{2}\right.} J^{(0)} J_{\left.\alpha_{3} \alpha_{4}\right)}^{(1)}-2 J^{(0)} \partial_{\left(\alpha_{1} \alpha_{2}\right.} J_{\left.\alpha_{3} \alpha_{4}\right)}^{(1)}\right]
$$


For spins $s=4$ and 5, we find:

$$
\begin{aligned}
& \partial^{\mu} J_{\mu \alpha_{1} \cdots \alpha_{6}}^{(4)} \sim \frac{1}{k}\left[8 \partial_{\left(\alpha_{1}\right.}{ }^{\beta} J_{\beta \alpha_{2}}^{(1)} \partial_{\alpha_{3} \alpha_{4}} J_{\left.\alpha_{5} \alpha_{6}\right)}^{(1)}+5 J_{\left(\alpha_{1} \alpha_{2}\right.}^{(1)} \partial_{\alpha_{3} \alpha_{4}} \partial_{\alpha_{5}}{ }^{\beta} J_{\left.\alpha_{6}\right) \beta}^{(1)}+J_{\left(\alpha_{1}\right.}^{(1) \beta} \partial_{\alpha_{2} \beta} \partial_{\alpha_{3} \alpha_{4}} J_{\left.\alpha_{5} \alpha_{6}\right)}^{(1)}\right. \\
& \left.+5 \partial_{\left(\alpha_{1} \alpha_{2}\right.} J^{(0)} J_{\left.\alpha_{3} \alpha_{4} \alpha_{5} \alpha_{6}\right)}^{(2)}-2 J^{(0)} \partial_{\left(\alpha_{1} \alpha_{2}\right.} J_{\left.\alpha_{3} \alpha_{4} \alpha_{5} \alpha_{6}\right)}^{(2)}\right],
\end{aligned}
$$

and

$$
\begin{aligned}
& \partial^{\mu} J_{\mu \alpha_{1} \cdots \alpha_{8}}^{(5)} \sim \frac{1}{k}\left[-160 \partial_{\left(\alpha_{1} \alpha_{2}\right.} \partial_{\alpha_{3}}{ }^{\beta} J_{\beta \alpha_{4}}^{(1)} J_{\left.\alpha_{5} \cdots \alpha_{8}\right)}^{(2)}-40 \partial_{\left(\alpha_{1} \alpha_{2}\right.} \partial_{\alpha_{3} \alpha_{4}} J_{\alpha_{5}}^{(1) \beta} J_{\left.\alpha_{6} \alpha_{7} \alpha_{8}\right) \beta}^{(2)}\right.
\end{aligned}
$$

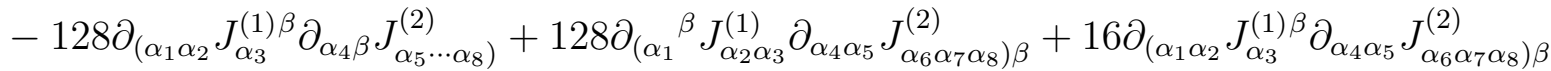

$$
\begin{aligned}
& +64 J_{\left(\alpha_{1}\right.}^{(1) \beta} \partial_{\beta \alpha_{2}} \partial_{\alpha_{3} \alpha_{4}} J_{\left.\alpha_{5} \cdots \alpha_{8}\right)}^{(2)}-56 J_{\left(\alpha_{1}\right.}^{(1) \beta} \partial_{\alpha_{2} \alpha_{3}} \partial_{\alpha_{4} \alpha_{5}} J_{\left.\alpha_{6} \alpha_{7} \alpha_{8}\right) \beta}^{(2)}+25 \partial_{\left(\alpha_{1} \alpha_{2}\right.} \partial_{\alpha_{3} \alpha_{4}} \partial_{\alpha_{5} \alpha_{6}} J^{(0)} J_{\left.\alpha_{7} \alpha_{8}\right)}^{(1)} \\
& -100 \partial_{\left(\alpha_{1} \alpha_{2}\right.} \partial_{\alpha_{3} \alpha_{4}} J^{(0)} \partial_{\alpha_{5} \alpha_{6}} J_{\left.\alpha_{7} \alpha_{8}\right)}^{(1)}+75 \partial_{\left(\alpha_{1} \alpha_{2}\right.} J^{(0)} \partial_{\alpha_{3} \alpha_{4}} \partial_{\alpha_{5} \alpha_{6}} J_{\left.\alpha_{7} \alpha_{8}\right)}^{(1)}-10 J^{(0)} \partial_{\left(\alpha_{1} \alpha_{2}\right.} \partial_{\alpha_{3} \alpha_{4}} \partial_{\alpha_{5} \alpha_{6}} J_{\left.\alpha_{7} \alpha_{8}\right)}^{(1)} \\
& \left.+140 \partial_{\left(\alpha_{1} \alpha_{2}\right.} J^{(0)} J_{\left.\alpha_{3} \cdots \alpha_{8}\right)}^{(3)}-40 J^{(0)} \partial_{\left(\alpha_{1} \alpha_{2}\right.} J_{\left.\alpha_{3} \cdots \alpha_{8}\right)}^{(3)}\right]
\end{aligned}
$$

In writing the above expressions, we have omitted an overall normalization constant as well as possible triple trace terms.

\section{F Perturbative Analysis of Anomalous Dimensions}

\section{F.1 Vanishing anomalous dimension of the scalar primary at planar level}

In this subsection, we check explicitly the vanishing of the anomalous dimension of the scalar operator $\bar{\psi} \psi$ at two-loop planar level, as previously argued based on the operator identities relating divergences of currents to double trace operators. The simplest way to do this computation is in the lightcone gauge, where there is no cubic gauge interaction vertex, and the planar two-loop fermion self-energy is finite. There are two diagrams that contribute to the logarithmic divergence.
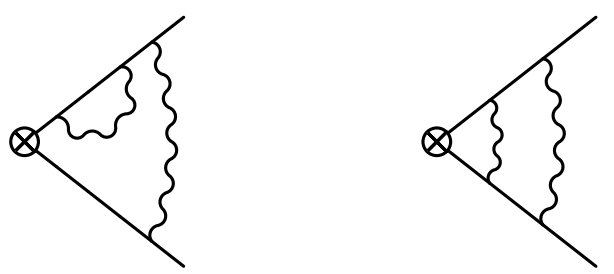
The first one (which comes with an additional factor of 2) is computed as

$$
\begin{aligned}
& -4 \pi \lambda^{2} \int \frac{d^{3} p}{(2 \pi)^{3}} \frac{\left|p_{s}\right|\left(\gamma^{3} p \gamma^{+}-\gamma^{+} p \gamma^{3}\right)}{\left(p^{2}\right)^{2}} \frac{p^{-}}{p_{s}^{2}}=4 \pi \lambda^{2} \int \frac{d^{3} p}{(2 \pi)^{3}} \frac{\left|p_{s}\right|}{\left(p^{2}\right)^{2}} \\
& =4 \pi \lambda^{2} \int_{0}^{1} d x \frac{x(1-x)^{-\frac{3}{2}}}{B\left(2,-\frac{1}{2}\right)} \int \frac{d^{3} p}{(2 \pi)^{3}} \frac{1}{\left(p_{s}^{2}+x p_{3}^{2}\right)^{\frac{3}{2}}} \\
& =4 \pi \lambda^{2} \frac{B\left(\frac{3}{2},-\frac{1}{2}\right)}{B\left(2,-\frac{1}{2}\right)} \frac{\ln \Lambda}{2 \pi^{2}} \\
& =\frac{\lambda^{2}}{2} \ln \Lambda .
\end{aligned}
$$

The second diagram (the rainbow correction to the vertex) is computed as

$$
\begin{aligned}
& -(4 \pi \lambda)^{2} \int \frac{d^{3} p d^{3} q}{(2 \pi)^{6}} \frac{2 p^{-} q^{-}\left(\gamma^{+} \not p \gamma^{+} \gamma^{3} \not p \gamma^{3}-\gamma^{3} \not p \gamma^{+} \gamma^{3} \not p \gamma^{+}\right)}{(p+q)^{2}\left(p^{2}\right)^{2} p_{s}^{2} q_{s}^{2}} \\
& =(4 \pi \lambda)^{2} \int \frac{d^{3} p d^{3} q}{(2 \pi)^{6}} \frac{4 p^{+} q^{-}}{(p+q)^{2}\left(p^{2}\right)^{2} q_{s}^{2}} \\
& =(4 \pi \lambda)^{2} \int_{0}^{1} d x \int \frac{d^{3} p d^{3} q}{(2 \pi)^{6}} \frac{4 p^{+}\left(q^{-}-x p^{-}\right)}{\left(p^{2}\right)^{2}\left[q_{s}^{2}+p_{s}^{2} x(1-x)+q_{3}^{2} x\right]^{2}} \\
& =-8 \pi \lambda^{2} \int \frac{d^{3} p}{(2 \pi)^{3}} \frac{\left|p_{s}\right|}{\left(p^{2}\right)^{2}}
\end{aligned}
$$

It indeed cancels twice of the previous diagram.

\section{F.2 Anomalous dimension of currents at order $1 / N$}

It has been argued that the higher spin currents $J_{s}(x)$ as well as the single trace scalar operator $\bar{\psi} \psi$ receives no anomalous dimension in the infinite $N$ limit. They do acquire anomalous dimension at subleading orders in $1 / N$. For the currents of nonzero spins, this can be understood in terms of the mixing of the divergence of the current with double trace operators. In this subsection, we perform a direct two-loop computation of the anomalous dimension of these currents at order $\lambda^{2} / N$. There are three diagrams that contribute, all of which involve a matter-loop-corrected gauge field propagator (which is suppressed by $1 / N$ because the matter is in the fundamental representation). These diagrams are listed below: 


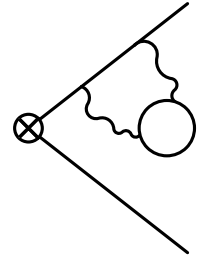

(a)

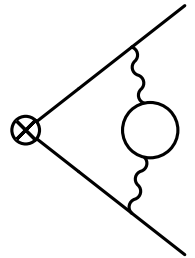

(b)

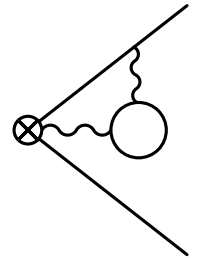

$(c)$

In the case of the scalar operator $\bar{\psi} \psi$, only the first two diagrams $(a),(b)$ contribute.

To begin, let us compute the matter loop correction to the gauge field self energy. This is given by

$$
\begin{aligned}
\Sigma_{G}^{\mu \nu}(q) & =-\frac{1}{2}(-i)^{2} \int \frac{d^{3} q}{(2 \pi)^{3}} \frac{\operatorname{Tr}\left(\gamma^{\mu} \not k \gamma^{\nu}(\not k+\not q)\right)}{k^{2}(k+q)^{2}} \\
& =\frac{q^{2} \delta^{\mu \nu}-q^{\mu} q^{\nu}}{32 q} .
\end{aligned}
$$

The factor $\frac{1}{2}$ is due to our normalization convention for the gauge group generators. The contribution to the fermion self energy by the matter-loop-corrected gauge propagator, involved in diagram $(a)$, is given by

$$
\Sigma^{\prime}(p)=\frac{N}{2} \int \frac{d^{3} q}{(2 \pi)^{3}} \frac{-i \gamma^{\mu}(\not p+\not q) \gamma^{\nu}}{(p+q)^{2}} D_{\mu \rho}(q) \Sigma_{G}^{\rho \sigma}(q) D_{\sigma \nu}(q)=i \frac{\lambda}{k} a_{1} \not p \ln \Lambda+\text { finite }
$$

where $D_{\mu \nu}(q)$ is the classical gauge propagator. Define the matter loop contribution to the gauge propagator as

$$
G_{\mu \nu}^{\prime}(q)=D_{\mu \rho}(q) \Sigma_{G}^{\rho \sigma}(q) D_{\sigma \nu}(q)
$$

Let us consider for now the anomalous dimension of $\bar{\psi} \psi$. The diagram $(b)$ is computed as

$$
-\frac{N}{2} \int \frac{d^{3} q}{(2 \pi)^{3}} \frac{D_{\mu \rho}(q) \Sigma_{G}^{\rho \sigma}(q) D_{\sigma}{ }^{\mu}(q)}{q^{2}}=-\frac{N}{2} \int \frac{d^{3} q}{(2 \pi)^{3}} \frac{1}{q^{2}} G_{\mu}^{\prime}{ }^{\mu}(q)=\frac{\lambda}{k} a_{2} \ln \Lambda
$$

The anomalous dimension is then given by

$$
\delta(\bar{\psi} \psi)=\frac{\lambda^{2}}{N}\left(a_{1}+a_{2}\right)
$$

The details of the computation of $a_{1}, a_{2}$ is given in F.3 (using Feynman gauge, temporal gauge, and lightcone gauge respectively). The result is $a_{1}+a_{2}=\frac{1}{3}$. 


\section{F.3 Two-loop non-planar anomalous dimension of the scalar primary}

\section{F.3.1 Feynman gauge}

In Feynman gauge, we have

$$
D_{\mu \nu}(q)=-\frac{4 \pi}{k} \epsilon_{\mu \nu \rho} \frac{q^{\rho}}{q^{2}},
$$

and so

$$
G_{\mu \nu}^{\prime}(q)=-\frac{\pi^{2}}{k^{2}} \frac{q^{2} \delta_{\mu \nu}-q_{\mu} q_{\nu}}{2 q^{3}} .
$$

Its contribution to the fermion self energy is

$$
\begin{aligned}
\Sigma^{\prime}(p) & =i \frac{N \pi^{2}}{4 k^{2}} \int \frac{d^{3} q}{(2 \pi)^{3}} \frac{q^{2} \gamma^{\mu}(\not p+\not 1) \gamma_{\mu}-\not \phi(p+\not) \phi}{q^{3}(p+q)^{2}} \\
& =i \frac{N \pi^{2}}{4 k^{2}} \int \frac{d^{3} q}{(2 \pi)^{3}} \frac{-2 q \cdot(p+q) \phi}{q^{3}(p+q)^{2}} \\
& =i \frac{N \pi^{2}}{4 k^{2}} \int \frac{d^{3} q}{(2 \pi)^{3}} \frac{p^{2}-q^{2}}{q^{3}(p+q)^{2}} \not
\end{aligned}
$$

The logarithmic divergent part is

$$
\begin{aligned}
& -i \frac{N \pi^{2}}{4 k^{2}} \int \frac{d^{3} q}{(2 \pi)^{3}} \frac{\not 1}{q(p+q)^{2}} \\
& =-i \frac{N \pi^{2}}{4 k^{2}} \frac{1}{B\left(1, \frac{1}{2}\right)} \int_{0}^{1} d x(1-x)^{-\frac{1}{2}} \int \frac{d^{3} q}{(2 \pi)^{3}} \frac{\not 1-x \not p}{\left[q^{2}+p^{2} x(1-x)\right]^{\frac{3}{2}}} \\
& \sim \frac{i N}{12 k^{2}} \not p \ln \Lambda .
\end{aligned}
$$

The diagram $(b)$ is computed as

$$
\frac{N \pi^{2}}{4 k^{2}} \int \frac{d^{3} q}{(2 \pi)^{3}} \frac{2}{q^{3}}=\frac{N}{4 k^{2}} \ln \Lambda .
$$

Therefore we find

$$
a_{1}+a_{2}=\frac{1}{12}+\frac{1}{4}=\frac{1}{3}
$$

\section{F.3.2 Temporal gauge}

In temporal gauge, we have

$$
D_{i j}(q)=\frac{4 \pi}{k} \epsilon_{i j} \frac{q^{3}}{\left(q^{3}\right)^{2}+\epsilon^{2}},
$$


and

$$
G_{i j}^{\prime}(q)=-\frac{\pi^{2}}{2 k^{2}} \frac{\left(q^{3}\right)^{2} \delta_{i j}+q_{i} q_{j}}{q} \frac{1}{\left(q^{3}\right)^{2}+\epsilon^{2}} .
$$

Its contribution to the fermion self energy is

$$
\begin{aligned}
\Sigma^{\prime}(p)= & i \frac{N \pi^{2}}{4 k^{2}} \int \frac{d^{3} q}{(2 \pi)^{3}} \frac{\left(q^{3}\right)^{2} \gamma_{i}(\not p+\not q) \gamma_{i}+\not q_{s}(\not p+\not q) \phi_{s}}{(p+q)^{2} q\left[\left(q^{3}\right)^{2}+\epsilon^{2}\right]} \\
= & i \frac{N \pi^{2}}{4 k^{2}} \int \frac{d^{3} q}{(2 \pi)^{3}} \frac{-2\left(q^{3}\right)^{2}\left(p^{3}+q^{3}\right) \gamma^{3}+2 q_{s} \cdot\left(p_{s}+q_{s}\right) \not_{s}-q_{s}^{2}(\not p+\not q)}{(p+q)^{2} q\left[\left(q^{3}\right)^{2}+\epsilon^{2}\right]} \\
= & i \frac{N \pi^{2}}{4 k^{2}} \int \frac{d^{3} q}{(2 \pi)^{3}} \frac{\left((p+q)^{2}-\left(p_{3}+q_{3}\right)^{2}-p_{s}^{2}\right) \phi_{s}-\left(q^{2}-q_{3}^{2}\right)\left(\not p_{s}+(p+q)^{0} \gamma^{3}\right)-2\left(q^{3}\right)^{2}\left(p^{3}+q^{3}\right) \gamma^{3}}{(p+q)^{2} q\left[\left(q^{3}\right)^{2}+\epsilon^{2}\right]} \\
= & i \frac{N \pi^{2}}{4 k^{2}} \int \frac{d^{3} q}{(2 \pi)^{3}}\left[\frac{\not q_{s}}{q\left(\left(q^{3}\right)^{2}+\epsilon^{2}\right)}+\frac{\not p_{s}-(p+q)^{0} \gamma^{3}}{(p+q)^{2} q}\right. \\
& \left.\quad-\frac{\not_{s}}{(p+q)^{2} q} \frac{\left(p_{3}+q_{3}\right)^{2}+p_{s}^{2}}{\left(q^{3}\right)^{2}+\epsilon^{2}}-\frac{|q|}{(p+q)^{2}} \frac{\not p_{s}+(p+q)^{0} \gamma^{3}}{\left(q^{3}\right)^{2}+\epsilon^{2}}\right] .
\end{aligned}
$$

The first term gives zero. The integrals of the remaining three terms give logarithmic divergences

$$
\left(\frac{\not p_{s}}{2}-\frac{p_{3} \gamma_{3}}{6}\right) \frac{\ln \Lambda}{\pi^{2}}, \quad \frac{\not p_{s}}{3 \pi^{2}} \ln \Lambda, \quad\left(\frac{\not p_{s}}{2}+\frac{3}{2} p_{3} \gamma_{3}\right) \frac{\ln \Lambda}{\pi^{2}} .
$$

Adding these together, we find

$$
\Sigma^{\prime}(p)=i \frac{N \ln \Lambda}{3 k^{2}} \not p+\text { finite. }
$$

The diagram $(b)$ is computed as

$$
\frac{N \pi^{2}}{4 k^{2}} \int \frac{d^{3} q}{(2 \pi)^{3}} \frac{\left(q^{3}\right)^{2}+q^{2}}{q^{3}} \frac{1}{\left(q^{3}\right)^{2}+\epsilon^{2}} \rightarrow 0 .
$$

The result agrees with the Feynman gauge computation.

\section{F.3.3 Lightcone gauge}

In lightcone gauge, we have

$$
D_{0+}(q)=-D_{+0}(q)=\frac{8 \pi i}{k} \frac{q^{-}}{q_{s}^{2}+i \epsilon}
$$

and

$$
\left(\begin{array}{cc}
G_{00}^{\prime}(q) & G_{0+}^{\prime}(q) \\
G_{+0}^{\prime}(q) & G_{++}^{\prime}(q)
\end{array}\right)=\frac{2 \pi^{2}}{k^{2}} \frac{\left(q^{-}\right)^{2}}{q\left(q_{s}^{2}+i \epsilon\right)^{2}}\left(\begin{array}{cc}
-\left(q^{+}\right)^{2} & q^{3} q^{+} \\
q^{3} q^{+} & q_{s}^{2}
\end{array}\right)
$$


Its contribution to the fermion self energy is

$$
\begin{aligned}
& \Sigma^{\prime}(p)=-i \frac{N \pi^{2}}{k^{2}} \int \frac{d^{3} q}{(2 \pi)^{3}}\left(q^{-}\right)^{2} \\
& \times \frac{-\left(q^{+}\right)^{2} \gamma^{3}(\not p+\not q) \gamma^{3}+q^{3} q^{+} \gamma^{3}(\not p+\not q) \gamma^{+}+q^{3} q^{+} \gamma^{+}(\not p+\not q) \gamma^{3}+q_{s}^{2} \gamma^{+}(\not p+\not q) \gamma^{+}}{(p+q)^{2} q\left(q_{s}^{2}+i \epsilon\right)^{2}} \\
&=-i \frac{N \pi^{2}}{2 k^{2}} \int \frac{d^{3} q}{(2 \pi)^{3}} q^{-} \frac{q^{+}\left[\not p+\not q-2(p+q)^{0} \gamma^{3}\right]+2 q^{3}\left[(p+q)^{+} \gamma^{3}+(p+q)^{0} \gamma^{+}\right]+4 q^{-}(p+q)^{+} \gamma^{+}}{(p+q)^{2} q\left(q_{s}^{2}+i \epsilon\right)^{2}}
\end{aligned}
$$

With a little manipulation, this integral can be written as

$$
\begin{aligned}
\Sigma^{\prime}(p)=-i \frac{N \pi^{2}}{4 k^{2}} \int \frac{d^{3} q}{(2 \pi)^{3}}\left[\frac{\not p+\not q-2 p^{3} \gamma^{3}}{(p+q)^{2} q}+\frac{4|q| q^{-} \gamma^{+}}{(p+q)^{2} q_{s}^{2}}\right] \\
=-i \frac{N \pi^{2}}{4 k^{2}} \int_{0}^{1} d x \int \frac{d^{3} q}{(2 \pi)^{3}}\left[\frac{(1-x)^{-\frac{1}{2}}}{B\left(1, \frac{1}{2}\right)} \frac{(1-x) \not p-2 p^{3} \gamma^{3}}{\left[q^{2}+x(1-x) p^{2}\right]^{\frac{3}{2}}}\right. \\
\left.+\frac{(1-x)^{-\frac{3}{2}}}{B\left(1,-\frac{1}{2}\right)} \frac{4 q^{-} \gamma^{+}}{\left[q_{s}^{2}+2 x p_{s} \cdot q_{s}+x p_{s}^{2}+q_{3}^{2}+x(1-x) p_{3}^{2}\right]^{\frac{1}{2}} q_{s}^{2}}\right] \\
=-i \frac{N \pi^{2}}{4 k^{2}}\left[\left(\frac{1}{6 \pi^{2}} \not p-\frac{1}{\pi^{2}} p^{3} \gamma^{3}\right) \ln \Lambda\right. \\
\left.+\int_{0}^{1} d x \int \frac{d^{2} q}{4 \pi^{3}} \frac{(1-x)^{-\frac{3}{2}}}{B\left(1,-\frac{1}{2}\right)} \frac{4 q^{-} \gamma^{+}}{q_{s}^{2}} \ln \frac{\left[q_{s}^{2}+2 x p_{s} \cdot q_{s}+x p_{s}^{2}+x(1-x) p_{3}^{2}\right]^{\frac{1}{2}}}{\left[\frac{1}{x^{2}}\right.}\right] \\
=-i \frac{N \pi^{2}}{4 k^{2}}\left(\frac{1}{6 \pi^{2}} \not p-\frac{1}{\pi^{2}} p^{3} \gamma^{3}-\frac{2}{\pi^{2}} p^{-} \gamma^{+}\right) \ln \Lambda
\end{aligned}
$$

While this expression is not Lorentz invariant, it is not a gauge invariant quantity. Inserting it into a two-point function computation gives

$$
a_{1}=\frac{1}{4}\left(-\frac{1}{6}+\frac{1}{3}+\frac{2}{3}\right)=\frac{5}{24} .
$$

The diagram $(b)$ is computed as

$$
\frac{N \pi^{2}}{k^{2}} \int \frac{d^{3} q}{(2 \pi)^{3}} \frac{\left(q^{+} q^{-}\right)^{2}}{q^{3}\left(q_{s}^{2}+i \epsilon\right)^{2}}=\frac{N}{8 k^{2}} \ln \Lambda .
$$

We have

$$
a_{1}+a_{2}=\frac{5}{24}+\frac{1}{8}=\frac{1}{3} .
$$

Once again, this agrees with the result we found in Feynman and temporal gauge. 


\section{G Perturbative Analysis of Three Point Functions}

\section{G.1 Vanishing one-loop contribution to the three point func- tions of scalar operators}

In this subsection, we consider the three point function of the scalar operator $J_{0}(x)=$ $\frac{1}{\sqrt{N}} \bar{\psi}(x) \psi(x)$. The normalization is such that its two-point function does not scale with $N$. By conformal symmetry,

$$
\left\langle J_{0}\left(x_{1}\right) J_{0}\left(x_{2}\right) J_{0}\left(x_{3}\right)\right\rangle=\frac{1}{\sqrt{N}} \frac{C(\lambda)+\mathcal{O}(1 / N)}{x_{12}^{2} x_{23}^{2} x_{31}^{2}}
$$

Parity requires $C(\lambda)$ to be an odd function of $\lambda$. So if the three point function of $J_{0}$ is nonzero, it can only receive contribution from odd loop order. A priori, for instance, there could be a one-loop contribution of order $\mathcal{O}(\lambda)$ to $\left\langle J_{0} J_{0} J_{0}\right\rangle$. We will perform this computation explicitly and see that it is in fact zero.

It suffices to take the limit $x_{12} \rightarrow 0$ while keeping $x_{13}$ finite. In this limit, we expect

$$
\left\langle J_{0}\left(x_{1}\right) J_{0}\left(x_{2}\right) J_{0}\left(x_{3}\right)\right\rangle \rightarrow \frac{1}{\sqrt{N}} \frac{C(\lambda)}{x_{12}^{2} x_{13}^{4}} .
$$

There are four distinct diagrams to consider (as shown in Fig. 3):

(A1) 1-loop self-energy correction to the fermion propagator $\left\langle\psi\left(x_{1}\right) \bar{\psi}\left(x_{2}\right)\right\rangle$.

(A2) 1-loop self-energy correction to the fermion propagator $\left\langle\psi\left(x_{1}\right) \bar{\psi}\left(x_{3}\right)\right\rangle$ (or $\left.\left\langle\psi\left(x_{2}\right) \bar{\psi}\left(x_{3}\right)\right\rangle\right)$. Such diagrams do not contribute to terms like $x_{12}^{-2} x_{13}^{-4}$, and will henceforth be ignored.

(B1) a gauge propagator correcting the vertex of $J_{0}\left(x_{3}\right)$.

(B2) a gauge propagator correcting the vertex of $J_{0}\left(x_{1}\right)\left(\right.$ or $\left.J_{0}\left(x_{1}\right)\right)$.

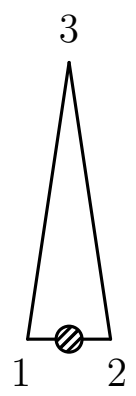

$A 1$

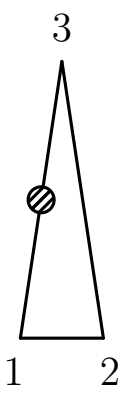

$A 2$

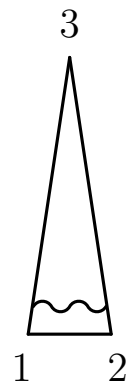

$B 1$

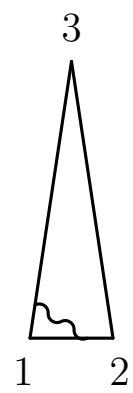

$B 2$

Figure 3

We will work in position space. Let $y_{1}, y_{2}$ be the two ends of the gauge propagator. In the limit $x_{12} \rightarrow 0$, the relevant contribution comes from $y_{1}, y_{2} \sim \mathcal{O}\left(x_{12}\right)$. From now 
we will set $x_{1}=0$ and $x_{2}=x$, while taking $x_{3}$ to be large. The contribution from diagrams (A1), (B1) and (B2) (note that (B2) contribution should be multiplied by a factor of 2 since there are two diagrams giving identical contributions) are proportional to (the overall $x_{3}$ dependent factor $\left|x_{3}\right|^{-4}$ is factored out)

$$
\begin{aligned}
& I(A 1)=\int d^{3} y_{1} d^{3} y_{2}\left\langle A_{\mu}\left(y_{1}\right) A_{\nu}\left(y_{2}\right)\right\rangle \operatorname{Tr}\left[\left\langle\psi(0) \bar{\psi}\left(y_{1}\right)\right\rangle \gamma^{\mu}\left\langle\psi\left(y_{1}\right) \bar{\psi}\left(y_{2}\right)\right\rangle \gamma^{\nu}\left\langle\psi\left(y_{2}\right) \bar{\psi}(x)\right\rangle\right], \\
& I(B 1)=\int d^{3} y_{1} d^{3} y_{2}\left\langle A_{\mu}\left(y_{1}\right) A_{\nu}\left(y_{2}\right)\right\rangle \operatorname{Tr}\left[\gamma^{\mu}\left\langle\psi\left(y_{1}\right) \bar{\psi}(0)\right\rangle\langle\psi(0) \bar{\psi}(x)\rangle\left\langle\psi(x) \bar{\psi}\left(y_{2}\right)\right\rangle \gamma^{\nu}\right], \\
& I(B 2)=\int d^{3} y_{1} d^{3} y_{2}\left\langle A_{\mu}\left(y_{1}\right) A_{\nu}\left(y_{2}\right)\right\rangle \operatorname{Tr}\left[\gamma^{\mu}\left\langle\psi\left(y_{1}\right) \bar{\psi}(0)\right\rangle\left\langle\psi(0) \bar{\psi}\left(y_{2}\right)\right\rangle \gamma^{\nu}\left\langle\psi\left(y_{2}\right) \bar{\psi}(x)\right\rangle\right] .
\end{aligned}
$$

In the following two subsubsections, we demonstrate the computation in Feynman gauge and in light cone gauge, respectively.

\section{G.1.1 Feynman gauge}

The propagators are

$$
\left\langle A_{\mu}(x) A_{\nu}(0)\right\rangle=-\frac{i}{k} \epsilon_{\mu \nu \rho} \frac{x^{\rho}}{|x|^{3}}, \quad\langle\psi(x) \bar{\psi}(0)\rangle=\frac{\not}{4 \pi|x|^{3}} .
$$

The results for the loop integrals are

$$
I(A 1)=-\frac{\lambda}{2 \pi x^{2}}, \quad I(B 1)=\frac{\lambda}{2 \pi x^{2}}, \quad I(B 2)=0 .
$$

Indeed they cancel, as claimed.

\section{G.1.2 Light cone gauge}

We have seen that the 1-loop correction to fermion propagator in light cone gauge is proportional to $\left|x^{0}\right| /|x|^{3}$. Without loss of generality, we can take the Euclidean time direction to be orthogonal to the plane spanned by $x_{1}, x_{2}, x_{3}$, and so diagrams (A1), 
(A2) simply vanish in the light cone gauge. (B1) and (B2) are then computed as

$$
\begin{aligned}
& I(B 1)=-\frac{\lambda}{(4 \pi)^{3}} \int d^{3} y_{1} d^{3} y_{2} \frac{\delta\left(y_{12}^{0}\right) y_{12}^{-}}{y_{12}^{2}} \frac{\operatorname{Tr}\left\{y_{1} \not \partial\left(y_{2}-\not x\right)\left[\gamma^{+}, \gamma^{3}\right]\right\}}{\left|y_{1}\right|^{3}\left|y_{2}-x\right|^{3}|x|^{3}} \\
& =-\frac{\lambda}{(4 \pi)^{3}} \int d^{3} y_{1} d^{3} y_{2} \frac{\delta\left(y_{12}^{0}\right)}{y_{12}^{2}} \frac{\operatorname{Tr}\left\{y_{1} \not \partial\left(y_{2}-\not x\right) y_{12}\right\}}{\left|y_{1}\right|^{3}\left|y_{2}-x\right|^{3}|x|^{3}} \\
& =-\frac{\lambda}{(4 \pi)^{3}} 2 \int d^{2} z_{1} d^{2} z_{2} d t \frac{1}{z_{12}^{2}} \frac{z_{1} \cdot x z_{12} \cdot\left(z_{1}-x\right)+z_{1} \cdot z_{12} x \cdot\left(z_{2}-x\right)-z_{12} \cdot x\left[z_{1} \cdot\left(z_{2}-x\right)+t^{2}\right]}{\left(z_{1}^{2}+t^{2}\right)^{\frac{3}{2}}\left(\left(z_{2}-x\right)^{2}+t^{2}\right)^{\frac{3}{2}}|x|^{3}} \\
& =-\frac{\lambda}{(4 \pi)^{3}} 2 \pi \int_{0}^{1} d a \int d^{2} z_{2} d t \frac{\left(a z_{2}^{2}+3 t^{2}\right) x \cdot\left(z_{2}-x\right)+t^{2} x^{2}}{\left(a z_{2}^{2}+t^{2}\right)^{\frac{3}{2}}\left(\left(z_{2}-x\right)^{2}+t^{2}\right)^{\frac{3}{2}}|x|^{3}} \\
& =0 \text {; } \\
& I(B 2)=\frac{\lambda}{(4 \pi)^{3}} \int d^{3} y_{1} d^{3} y_{2} \frac{\delta\left(y_{12}^{0}\right) y_{12}^{-}}{y_{12}^{2}} \frac{\operatorname{Tr}\left\{\left(\gamma^{3} y_{1} y_{2} \gamma^{+}-\gamma^{+} y_{1} y_{2} \gamma^{3}\right)\left(y_{2}-\not x\right)\right\}}{\left|y_{1}\right|^{3}\left|y_{2}\right|^{3}\left|y_{2}-x\right|^{3}} \\
& =-\frac{\lambda}{(4 \pi)^{3}} 2 \int d^{3} y_{1} d^{3} y_{2} \frac{\delta\left(y_{12}^{0}\right)}{y_{12}^{2}} \frac{\left(y_{1} \cdot y_{2}\right) y_{12} \cdot\left(y_{2}-x\right)}{\left|y_{1}\right|^{3}\left|y_{2}\right|^{3}\left|y_{2}-x\right|^{3}} \\
& =-\frac{\lambda}{(4 \pi)^{3}} 2 \int d^{2} z_{1} d^{2} z_{2} d t \frac{1}{z_{12}^{2}} \frac{\left(z_{1} \cdot z_{2}+t^{2}\right) z_{12} \cdot\left(z_{2}-x\right)}{\left(z_{1}^{2}+t^{2}\right)^{\frac{3}{2}}\left(z_{2}^{2}+t^{2}\right)^{\frac{3}{2}}\left(\left(z_{2}-x\right)^{2}+t^{2}\right)^{\frac{3}{2}}} \\
& =0 \text {. }
\end{aligned}
$$

We see that the contribution from each diagram vanishes individually in the light cone gauge.

\section{G.2 One-loop parity violating structure of $\langle J J J\rangle$}

We will work in temporal gauge, which is particularly convenient for this computation since the position space gauge field propagator is very simple. With our special choice of null polarization vectors $\varepsilon_{1}=\varepsilon_{2}=\varepsilon_{3}=\varepsilon$, all diagrams involving a one-loop fermion self-energy vanish (Figure 4(a)). Similarly, the diagrams with a gauge propagator coming out of a vertex and ends on an adjacent fermion line also vanish (Figure 4(b)). We further set $x_{3}=x=t \hat{e}_{0}, \notin=\gamma^{+}, x_{1}=0, x_{2}=-\delta$ purely spatial, as explained earlier. There are three diagrams that a priori contribute to $\langle J J J\rangle$ in this special case ( $J$ is assumed to be a current of nonzero spin):

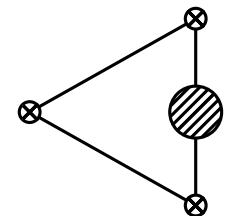

(a)

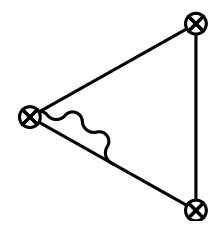

(b) 
Figure 4: diagrams that do not contribute to $\langle J J J\rangle$ in the special limit.

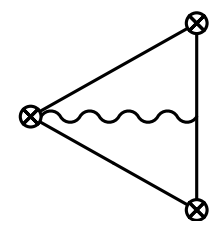

(a)

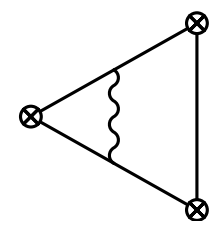

(b)

Figure 5: type of diagrams that contribute to $\langle J J J\rangle$ in the special limit.

(I) A gauge propagator coming out of the vertex $J\left(x_{2}\right)$ and ends on the fermion line between $x_{1}$ and $x_{3}$. This is shown in figure 5(a). Two analogous diagrams, in which the gauge field propagator comes out of $J\left(x_{1}\right)$ or $J\left(x_{3}\right)$, vanish identically because one encounters the structure $\notin k_{31} \notin=\not t \gamma^{3} \notin=0$ in the loop integral, for the special configuration of positions and polarizations we are considering here.

(II) A gauge propagator correcting the vertex $J\left(x_{3}\right)$. This is shown in figure $5(\mathrm{~b})$, where the vertex on the left is $J\left(x_{3}\right)$.

(III) a gauge propagator correcting the vertex $J\left(x_{1}\right)$. The diagram is the same as in figure $5(\mathrm{~b})$ except that the vertex on the left is $J\left(x_{1}\right)$ in this case. Note that the analogous diagram with the gauge propagator correcting $J\left(x_{2}\right)$ vanishes identically, because once again it involves the structure $\notin x_{31} \notin=\notin t \gamma^{3} \notin=0$.

The diagrams I,II,III are exhibited more explicitly in the limit $|\delta| \ll|t|$ in figure 6 .
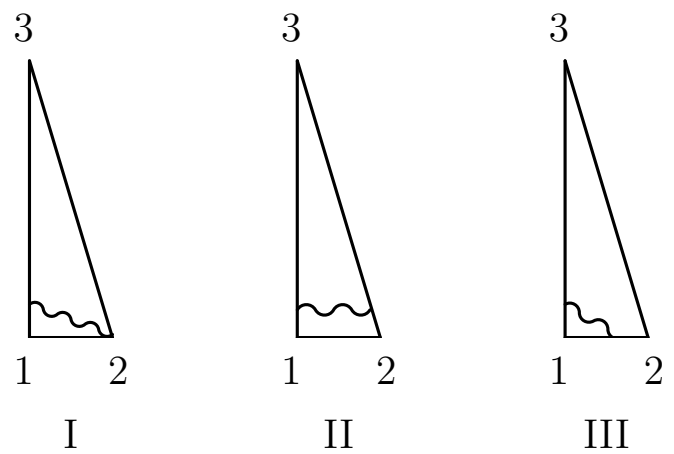

Figure 6

\section{G.2.1 $\langle j j T\rangle$}

To begin, let us consider the one-loop contribution to $\left\langle j_{\varepsilon}\left(x_{1}\right) j_{\varepsilon}\left(x_{2}\right) T_{\varepsilon}\left(x_{3}\right)\right\rangle$. For this correlator, diagram I does not contribute as $A_{\mu}$ does not appear explicitly in $j\left(x_{2}\right)$. 
Diagram II is given by

$$
\begin{aligned}
& \int d^{3} y_{1} d^{3} y_{2}\left\langle A_{i}\left(y_{1}\right) A_{j}\left(y_{2}\right)\right\rangle \operatorname{Tr}\left[\gamma^{j}\left\langle\psi\left(y_{2}\right) \bar{\psi}\left(x_{3}\right)\right\rangle \notin\left(\varepsilon \cdot \overleftrightarrow{\partial}_{x_{3}}\right)\left\langle\psi\left(x_{3}\right) \bar{\psi}\left(y_{1}\right)\right\rangle \gamma^{i}\left\langle\psi\left(y_{1}\right) \bar{\psi}\left(x_{1}\right)\right\rangle\right. \\
& \left.\quad \times \notin\left\langle\psi\left(x_{1}\right) \bar{\psi}\left(x_{2}\right)\right\rangle \notin\left\langle\psi\left(x_{2}\right) \bar{\psi}\left(y_{2}\right)\right\rangle\right]
\end{aligned}
$$

At our special configuration $x_{1}=0, x_{2}=-\delta, x_{3}=x=t \hat{e}_{0}(\delta$ purely spatial $), \notin=\gamma^{+}$, the loop integral is proportional to

$$
\begin{aligned}
& \int d^{3} y_{1} d^{3} y_{2} \epsilon\left(y_{12}^{0}\right) \delta^{2}\left(\vec{y}_{12}\right) \epsilon_{i j} \operatorname{Tr}\left[\gamma^{j}\left(y_{2}-\not x\right) \gamma^{+}\left(\not x-y_{1}\right) \gamma^{i} y_{1} \gamma^{+} \not \gamma^{+}\left(-y_{2}-\not \varnothing\right)\right] \\
& \quad \times \frac{1}{\left|y_{1}\right|^{3}|\delta|^{3}\left|\delta+y_{2}\right|^{3}}\left(\frac{1}{\left|x-y_{2}\right|^{3}} \overleftrightarrow{\partial}_{x^{-}} \frac{1}{\left|x-y_{1}\right|^{3}}\right) \\
& =-16 i \frac{\delta^{+}}{|\delta|^{3}} \int d^{2} z d t_{1} d t_{2} \epsilon\left(t_{12}\right)\left[\left(z^{+}\right)^{2} t_{2}\left(t-t_{2}\right)-z^{+}(z+\delta)^{+} t_{1}\left(t-t_{1}\right)\right] \\
& \quad \times \frac{1}{\left(z^{2}+t_{1}^{2}\right)^{\frac{3}{2}}\left[(\delta+z)^{2}+t_{2}^{2}\right]^{\frac{3}{2}}}\left\{\frac{1}{\left[\left(t-t_{2}\right)^{2}+z^{2}\right]^{\frac{3}{2}}} \overleftrightarrow{\partial}_{z^{-}} \frac{1}{\left[\left(t-t_{1}\right)^{2}+z^{2}\right]^{\frac{3}{2}}}\right\}
\end{aligned}
$$

In the small $\delta$ limit, the leading contribution to the integral comes from two domains:

$$
\begin{aligned}
& D_{1}: t_{1}, t_{2} \sim \mathcal{O}(\delta), \\
& D_{2}:\left(t_{1}-t\right), t_{2} \sim \mathcal{O}(\delta) .
\end{aligned}
$$


The contribution from region $D_{1}$ is easily seen from G.11) to vanish at order $\mathcal{O}\left(t^{-7}\right)$. The contribution from $D_{2}$ is

$$
\begin{aligned}
- & 16 i \frac{\delta^{+} \epsilon(t)}{|\delta|^{3}|t|^{3}} \int d^{2} z d t_{1}^{\prime} d t_{2} \frac{1}{\left[(\delta+z)^{2}+t_{2}^{2}\right]^{\frac{3}{2}}} \\
& \times\left\{\left[-\left(z^{+}\right)^{2} t_{2}^{2}+z^{+}(z+\delta)^{+} t_{1}^{\prime 2}-3 z^{+}(z+\delta)^{+} t_{1}^{\prime 2}\right]\left[\frac{1}{\left(t^{2}+z^{2}\right)^{\frac{3}{2}}} \overleftrightarrow{\partial}_{z^{-}} \frac{1}{\left(t_{1}^{\prime 2}+z^{2}\right)^{\frac{3}{2}}}\right]\right. \\
& \left.+\left[\left(z^{+}\right)^{2} t_{2} t-z^{+}(z+\delta)^{+} t_{1}^{\prime} t\right]\left[\frac{3 t t_{2}}{\left(t^{2}+z^{2}\right)^{\frac{5}{2}}} \overleftrightarrow{\partial}_{z^{-}} \frac{1}{\left(t_{1}^{\prime 2}+z^{2}\right)^{\frac{3}{2}}}\right]\right\} \cdot\left[1+\mathcal{O}\left(\frac{\delta^{2}}{t^{2}}\right)\right] \\
= & -16 i \frac{\delta^{+} \epsilon(t)}{|\delta|^{3} t^{6}} \int d^{2} z d t_{1}^{\prime} d t_{2} \frac{1}{\left[(\delta+z)^{2}+t_{2}^{2}\right]^{\frac{3}{2}}} \frac{-3 z^{+}}{\left(t_{1}^{\prime 2}+z^{2}\right)^{\frac{5}{2}}} \\
& \times\left\{\left[-\left(z^{+}\right)^{2} t_{2}^{2}-2 z^{+}(z+\delta)^{+} t_{1}^{\prime 2}\right]+3\left(z^{+}\right)^{2} t_{2}^{2}\right\} \cdot\left[1+\mathcal{O}\left(\frac{\delta^{2}}{t^{2}}\right)\right] \\
= & 32 i \frac{\delta^{+} \epsilon(t)}{|\delta|^{3} t^{6}} \int d^{2} z d t_{2} \frac{\left(z^{+}\right)^{2}\left[4 t_{2}^{2} z^{+}-2 z^{2}(z+\delta)^{+}\right]}{\left[(\delta+z)^{2}+t_{2}^{2}\right]^{\frac{3}{2}}|z|^{4}}\left[1+\mathcal{O}\left(\frac{\delta^{2}}{t^{2}}\right)\right] \\
= & 32 i \frac{\left(\delta^{+}\right)^{4} \epsilon(t)}{|\delta|^{3} t^{6}}\left\{\frac{1}{B\left(\frac{3}{2}, 2\right)} \int_{0}^{1} d a a^{\frac{1}{2}}(1-a) \int d^{2} z d t_{2} \frac{a^{2}\left[4 t_{2}^{2}(-a)\right]}{\left[z^{2}+a(1-a) \delta^{2}+a t_{2}^{2}\right]^{\frac{7}{2}}}\right. \\
& \left.+\frac{1}{B\left(\frac{3}{2}, 1\right)} \int_{0}^{1} d a a^{\frac{1}{2}} \int d^{2} z d t_{2} \frac{-2 a^{2}(1-a)}{\left[z^{2}+a(1-a) \delta^{2}+a t_{2}^{2}\right]^{\frac{5}{2}}}\right\}\left[1+\mathcal{O}\left(\frac{\delta^{2}}{t^{2}}\right)\right] \\
= & -128 \pi i \frac{\left(\delta^{+}\right)^{4} \epsilon(t)}{|\delta|^{5} t^{6}}\left[1+\mathcal{O}\left(\frac{\delta^{2}}{t^{2}}\right)\right] .
\end{aligned}
$$

Diagram III is given by

$$
\begin{aligned}
\int d^{3} y_{1} d^{3} y_{2}\left\langle A_{i}\left(y_{1}\right) A_{j}\left(y_{2}\right)\right\rangle \operatorname{Tr}\left[\gamma^{i}\left\langle\psi\left(y_{1}\right) \bar{\psi}\left(x_{1}\right)\right\rangle \notin\left\langle\psi\left(x_{1}\right) \bar{\psi}\left(y_{2}\right)\right\rangle \gamma^{j}\left\langle\psi\left(y_{2}\right) \bar{\psi}\left(x_{2}\right)\right\rangle\right. \\
\left.\quad \times \notin\left\langle\psi\left(x_{2}\right) \bar{\psi}\left(x_{3}\right)\right\rangle \notin\left(\varepsilon \cdot \overleftrightarrow{\partial}_{x_{3}}\right)\left\langle\psi\left(x_{3}\right) \bar{\psi}\left(y_{1}\right)\right\rangle\right]
\end{aligned}
$$

The loop integral is evaluated as

$$
\begin{aligned}
\int & d^{3} y_{1} d^{3} y_{2} \epsilon\left(y_{12}^{0}\right) \delta^{2}\left(\vec{y}_{12}\right) \epsilon_{i j} \operatorname{Tr}\left[\gamma^{i} \ddot{y}_{1} \gamma^{+}\left(-y_{2}\right) \gamma^{j}\left(y_{2}+\not\right) \gamma^{+}(-\not x-\not) \gamma^{+}\left(\not x-y_{1}\right)\right] \\
& \times \frac{1}{\left|y_{1}\right|^{3}\left|y_{2}\right|^{3}\left|\delta+y_{2}\right|^{3}}\left(\frac{1}{\left|x-y_{1}\right|^{3}} \overleftrightarrow{\partial}_{x^{-}} \frac{1}{|x+\delta|^{3}}\right) \\
= & -16 i \delta^{+} \int d^{2} z d t_{1} d t_{2} \epsilon\left(t_{12}\right)\left[\left(z^{+}\right)^{2} t_{2}^{2}+z^{+}(z+\delta)^{+} t_{1}\left(t-t_{1}\right)\right] \\
& \times \frac{1}{\left(z^{2}+t_{1}^{2}\right)^{\frac{3}{2}}\left(z^{2}+t_{2}^{2}\right)^{\frac{3}{2}}\left[(z+\delta)^{2}+t_{2}^{2}\right]^{\frac{3}{2}}}\left\{\frac{1}{\left[\left(t-t_{1}\right)^{2}+z^{2}\right]^{\frac{3}{2}}}\left(\overleftarrow{\partial}_{z^{-}}+\vec{\partial}_{\delta^{-}}\right) \frac{1}{\left(t^{2}+\delta^{2}\right)^{\frac{3}{2}}}\right\}
\end{aligned}
$$


In the small $\delta$ limit, the integral is again dominated by the contribution from the regions $D_{1}$ and $D_{2}$. The contribution from $D_{1}$ is

$$
\begin{aligned}
& -16 i \frac{\delta^{+}}{t^{7}} \int d^{2} z d t_{1} d t_{2} \epsilon\left(t_{12}\right) t_{1} z^{+}(z+\delta)^{+} \frac{-3(z+\delta)^{+}}{\left(z^{2}+t_{1}^{2}\right)^{\frac{3}{2}}\left(z^{2}+t_{2}^{2}\right)^{\frac{3}{2}}\left[(z+\delta)^{2}+t_{2}^{2}\right]^{\frac{3}{2}}} \\
& =96 i \frac{\delta^{+}}{t^{7}} \int d^{2} z d t_{2} \frac{z^{+}\left((z+\delta)^{+}\right)^{2}}{\left(z^{2}+t_{2}^{2}\right)^{2}\left[(z+\delta)^{2}+t_{2}^{2}\right]^{\frac{3}{2}}}=96 i \frac{\delta^{+}}{t^{7}} \int d^{3} y \frac{y^{+}\left((y+\delta)^{+}\right)^{2}}{|y|^{4}|y+\delta|^{3}} \\
& =96 i \frac{\left(\delta^{+}\right)^{4}}{t^{7}} \frac{1}{B\left(2, \frac{3}{2}\right)} \int_{0}^{1} d a a^{\frac{1}{2}}(1-a) \int d^{3} y \frac{(-a)(1-a)^{2}}{\left[y^{2}+\delta^{2} a(1-a)\right]^{\frac{7}{2}}} \\
& =-256 \pi i \frac{\left(\delta^{+}\right)^{4}}{\delta^{4} t^{7}}
\end{aligned}
$$

The contribution from $D_{2}$ is

$$
\begin{aligned}
& -16 i \frac{\delta^{+} \epsilon(t)}{|t|^{3}} \int d^{2} z d t_{1}^{\prime} d t_{2} \frac{\left(z^{+}\right)^{2} t_{2}^{2}+z^{+}(z+\delta)^{+} t_{1}^{\prime}\left(t-t_{1}^{\prime}\right)}{\left(z^{2}+\left(t-t_{1}^{\prime}\right)^{2}\right)^{\frac{3}{2}}\left(z^{2}+t_{2}^{2}\right)^{\frac{3}{2}}\left[(z+\delta)^{2}+t_{2}^{2}\right)^{\frac{3}{2}}} \frac{-3 z^{+}}{\left(t_{1}^{\prime 2}+z^{2}\right)^{\frac{5}{2}}}\left[1+\mathcal{O}\left(\frac{\delta^{2}}{t^{2}}\right)\right] \\
& =-16 i \frac{\delta^{+} \epsilon(t)}{t^{6}} \int d^{2} z d t_{1}^{\prime} d t_{2} \frac{\left(z^{+}\right)^{2} t_{2}^{2}-z^{+}(z+\delta)^{+} t_{1}^{\prime 2}+3 z^{+}(z+\delta)^{+} t_{1}^{\prime 2}}{\left(z^{2}+t_{2}^{2}\right)^{\frac{3}{2}}\left[(z+\delta)^{2}+t_{2}^{2}\right]^{\frac{3}{2}}} \frac{-3 z^{+}}{\left(t_{1}^{\prime 2}+z^{2}\right)^{\frac{5}{2}}}\left[1+\mathcal{O}\left(\frac{\delta^{2}}{t^{2}}\right)\right] \\
& =64 i \frac{\delta^{+} \epsilon(t)}{t^{6}} \int d^{2} z d t_{2}\left(z^{+}\right)^{2} \frac{z^{+} t_{2}^{2}+z^{2}(z+\delta)^{+}}{\left(z^{2}+t_{2}^{2}\right)^{\frac{3}{2}}\left[(z+\delta)^{2}+t_{2}^{2}\right]^{\frac{3}{2}}|z|^{4}}\left[1+\mathcal{O}\left(\frac{\delta^{2}}{t^{2}}\right)\right] \\
& =64 i \frac{\delta^{+} \epsilon(t)}{t^{6}} \frac{1}{B\left(\frac{3}{2}, \frac{3}{2}\right)} \int_{0}^{1} d a a^{\frac{1}{2}}(1-a)^{\frac{1}{2}} \int d^{2} z d t_{2}\left(z^{+}\right)^{2} \frac{z^{+} t_{2}^{2}+z^{2}(z+\delta)^{+}}{\left[(1-a) z^{2}+a(z+\delta)^{2}+t_{2}^{2}\right]^{3}|z|^{4}}\left[1+\mathcal{O}\left(\frac{\delta^{2}}{t^{2}}\right)\right] \\
& =8 \pi i \frac{\delta^{+} \epsilon(t)}{t^{6}} \frac{1}{B\left(\frac{3}{2}, \frac{3}{2}\right)} \int_{0}^{1} d a a^{\frac{1}{2}}(1-a)^{\frac{1}{2}} \\
& \times \int d^{2} z\left\{\frac{\left(z^{+}\right)^{3}}{|z|^{4}\left(z^{2}+2 a \delta \cdot z+a \delta^{2}\right)^{\frac{3}{2}}}+\frac{3\left(z^{+}\right)^{2}(z+\delta)^{+}}{z^{2}\left(z^{2}+2 a \delta \cdot z+a \delta^{2}\right)^{\frac{5}{2}}}\right\}\left[1+\mathcal{O}\left(\frac{\delta^{2}}{t^{2}}\right)\right] \\
& =8 \pi i \frac{\delta^{+} \epsilon(t)}{t^{6}} \frac{1}{B\left(\frac{3}{2}, \frac{3}{2}\right)} \int_{0}^{1} d a a^{\frac{1}{2}}(1-a)^{\frac{1}{2}} \int_{0}^{1} d b \int d^{2} z\left\{\frac{b^{\frac{1}{2}}(1-b)}{B\left(\frac{3}{2}, 2\right)}\left(z^{+}\right)^{3}+\frac{b^{\frac{3}{2}}}{B\left(\frac{5}{2}, 1\right)}\left[3\left(z^{+}\right)^{2}(z+\delta)^{+}\right]\right\} \\
& \times \frac{1}{\left[b\left(z^{2}+2 a \delta \cdot z+a \delta^{2}\right)+(1-b) z^{2}\right]^{\frac{7}{2}}}\left[1+\mathcal{O}\left(\frac{\delta^{2}}{t^{2}}\right)\right] \\
& =8 \pi i \frac{\left(\delta^{+}\right)^{4} \epsilon(t)}{t^{6}} \frac{1}{B\left(\frac{3}{2}, \frac{3}{2}\right)} \int_{0}^{1} d a a^{\frac{1}{2}}(1-a)^{\frac{1}{2}} \int_{0}^{1} d b\left\{\frac{b^{\frac{1}{2}}(1-b)}{B\left(\frac{3}{2}, 2\right)}(-a b)^{3}+\frac{b^{\frac{3}{2}}}{B\left(\frac{5}{2}, 1\right)}\left[3(a b)^{2}(1-a b)\right]\right\} \\
& \times \int d^{2} z \frac{1}{\left[z^{2}+a b(1-a b) \delta^{2}\right]^{\frac{7}{2}}}\left[1+\mathcal{O}\left(\frac{\delta^{2}}{t^{2}}\right)\right] \\
& =128 \pi i \frac{\left(\delta^{+}\right)^{4} \epsilon(t)}{|\delta|^{5} t^{6}}\left[1+\mathcal{O}\left(\frac{\delta^{2}}{t^{2}}\right)\right] \text {. }
\end{aligned}
$$


Putting back in normalization factors associated with the propagators, we obtain

$$
\langle j j T\rangle_{1-\text { loop }} \longrightarrow \frac{N \lambda}{2 \pi^{3}} \frac{\left(\delta^{+}\right)^{4}}{\delta^{4} t^{7}}
$$

in our limit.

\section{G.2.2 $\langle T T T\rangle$}

Next, we consider the one-loop contribution to $\left\langle T_{\varepsilon}\left(x_{1}\right) T_{\varepsilon}\left(x_{2}\right) T_{\varepsilon}\left(x_{3}\right)\right\rangle$. Diagram I is given by

$$
\begin{aligned}
& \int d^{3} y\left\langle A_{i}\left(x_{2}\right) A_{j}(y)\right\rangle \\
& \times \operatorname{Tr}\left[\gamma^{i}\left\langle\psi(y) \bar{\psi}\left(x_{1}\right)\right\rangle \notin\left(\varepsilon \cdot \overleftrightarrow{\partial}_{x_{1}}\right)\left\langle\psi\left(x_{1}\right) \bar{\psi}\left(x_{2}\right)\right\rangle \notin \varepsilon j\left\langle\psi\left(x_{2}\right) \bar{\psi}\left(x_{3}\right)\right\rangle \notin\left(\varepsilon \cdot \overleftrightarrow{\partial}_{x_{3}}\right)\left\langle\psi\left(x_{3}\right) \bar{\psi}(y)\right\rangle\right] .
\end{aligned}
$$

At our special configuration $x_{1}=0, x_{2}=-\delta, x_{3}=x=t \hat{e}_{0}(\delta$ purely spatial $), \notin=\gamma^{+}$, the loop integral is proportional to

$$
\begin{aligned}
& \int d^{3} y \epsilon\left(y^{0}\right) \delta^{2}\left(\vec{y}-\vec{x}_{2}\right) \epsilon_{i j} \xi^{j} \operatorname{Tr}\left[\gamma^{i}\left(\not y-\not x_{1}\right) \gamma^{+} \not x_{12} \gamma^{+} \not x_{23} \gamma^{+}\left(\not x_{3}-\not y\right)\right] \\
& \times\left(\frac{1}{\left|y-x_{1}\right|^{3}} \overleftrightarrow{\partial}_{x_{1}^{-}} \frac{1}{\left|x_{12}\right|^{3}}\right)\left(\frac{1}{\left|x_{23}\right|^{3}} \overleftrightarrow{\partial}_{x_{3}^{-}} \frac{1}{\left|x_{3}-y\right|^{3}}\right) \\
&=-\int d^{3} y \epsilon\left(y^{0}\right) \delta^{2}(\vec{y}+\vec{\delta}) \epsilon_{i j} \varepsilon^{j} \operatorname{Tr}\left[\gamma^{i} y \gamma^{+} \not \gamma^{+}(-\not b-\not x) \gamma^{+}(\not x-y)\right] \\
& \times \frac{9}{|y|^{3}|\delta|^{3}|x-y|^{3}|x+\delta|^{3}}\left(\frac{y^{+}}{y^{2}}+\frac{\delta^{+}}{\delta^{2}}\right)\left(\frac{y^{+}}{(x-y)^{2}}+\frac{\delta^{+}}{(x+\delta)^{2}}\right) \\
&=-9 \cdot 16 i \frac{\left(\delta^{+}\right)^{6}}{|\delta|^{3}|x+\delta|^{3}} \int d y^{0} \frac{\epsilon\left(y^{0}\right)}{\left(\delta^{2}+\left(y^{0}\right)^{2}\right)^{\frac{3}{2}}\left(\delta^{2}+\left(x^{0}-y^{0}\right)^{2}\right)^{\frac{3}{2}}}\left(\frac{1}{\delta^{2}+\left(y^{0}\right)^{2}}-\frac{1}{\delta^{2}}\right) \\
& \quad \times\left(\frac{1}{\left(x^{0}-y^{0}\right)^{2}+\delta^{2}}-\frac{1}{\left(x^{0}\right)^{2}+\delta^{2}}\right) \\
&=9 \cdot 16 i \frac{\left(\delta^{+}\right)^{6}}{|\delta|^{5}|x+\delta|^{5}} \int d y^{0} \frac{\epsilon\left(y^{0}\right)\left(y^{0}\right)^{3}\left(2 x^{0}-y^{0}\right)}{\left(\delta^{2}+\left(y^{0}\right)^{2}\right)^{\frac{5}{2}}\left(\delta^{2}+\left(x^{0}-y^{0}\right)^{2}\right)^{\frac{5}{2}}} .
\end{aligned}
$$

In the small $\delta$ limit, the integral is dominated by the contribution from $\left(x^{0}-y^{0}\right) \sim \mathcal{O}(\delta)$,

$$
\sim 9 \cdot 16 i \frac{\left(\delta^{+}\right)^{6}}{|\delta|^{5}|x|^{6}} \int d y^{0} \frac{\epsilon\left(x^{0}\right)}{\left(\delta^{2}+\left(x^{0}-y^{0}\right)^{2}\right)^{\frac{5}{2}}}\left[1+\mathcal{O}\left(\frac{\delta^{2}}{t^{2}}\right)\right]=-192 i \frac{\left(\delta^{+}\right)^{6} \epsilon(t)}{|\delta|^{9} t^{6}}\left[1+\mathcal{O}\left(\frac{\delta^{2}}{t^{2}}\right)\right]
$$

and from $y^{0} \sim \mathcal{O}(\delta)$,

$$
\sim 9 \cdot 16 i \frac{\left(\delta^{+}\right)^{6} 2 x^{0}}{|\delta|^{5}|x|^{10}} \int d y^{0} \frac{\epsilon\left(y^{0}\right)\left(y^{0}\right)^{3}}{\left(\delta^{2}+\left(y^{0}\right)^{2}\right)^{\frac{5}{2}}}=-192 i \frac{\left(\delta^{+}\right)^{6}}{|\delta|^{6} t^{9}} .
$$


The former is an artifact of temporal gauge, and will cancel against diagrams II, III. The latter is subleading and does not contribute to the overall coefficient of the parity odd $\langle T T T\rangle$ structure.

Diagram II is given by

$$
\begin{aligned}
\int d^{3} y_{1} d^{3} y_{2}\left\langle A_{i}\left(y_{1}\right) A_{j}\left(y_{2}\right)\right\rangle \operatorname{Tr}\left[\gamma^{j}\left\langle\psi\left(y_{2}\right) \bar{\psi}\left(x_{3}\right)\right\rangle \notin\left(\varepsilon \cdot \overleftrightarrow{\partial}_{x_{3}}\right)\left\langle\psi\left(x_{3}\right) \bar{\psi}\left(y_{1}\right)\right\rangle \gamma^{i}\left\langle\psi\left(y_{1}\right) \bar{\psi}\left(x_{1}\right)\right\rangle\right. \\
\left.\quad \times \notin\left(\varepsilon \cdot \overleftrightarrow{\partial}_{x_{1}}\right)\left\langle\psi\left(x_{1}\right) \bar{\psi}\left(x_{2}\right)\right\rangle \notin\left(\varepsilon \cdot \overleftrightarrow{\partial}_{x_{2}}\right)\left\langle\psi\left(x_{2}\right) \bar{\psi}\left(y_{2}\right)\right\rangle\right] .
\end{aligned}
$$

The loop integral is computed as

$$
\begin{aligned}
\int & d^{3} y_{1} d^{3} y_{2} \epsilon\left(y_{12}^{0}\right) \delta^{2}\left(\vec{y}_{12}\right) \epsilon_{i j} \operatorname{Tr}\left[\gamma^{j}\left(y_{2}-\not x\right) \gamma^{+}\left(\not x-y_{1}\right) \gamma^{i} y_{1} \gamma^{+} \not \gamma^{+}\left(-y_{2}-\not\right)\right] \\
& \times\left(\frac{1}{\left|x-y_{2}\right|^{3}} \overleftrightarrow{\partial}_{x^{-}} \frac{1}{\left|x-y_{1}\right|^{3}}\right)\left[\frac{1}{\left|y_{1}\right|^{3}}\left(\overleftarrow{\partial}_{y_{1}^{-}}+\vec{\partial}_{\delta^{-}}\right) \frac{1}{|\delta|^{3}}\left(\overleftarrow{\partial}_{\delta^{-}}-\vec{\partial}_{y_{2}^{-}}\right) \frac{1}{\left|\delta^{\prime}+y_{2}\right|^{3}}\right]_{\delta^{\prime}=\delta} \\
= & -16 i \delta^{+} \int d^{2} z d t_{1} d t_{2} \epsilon\left(t_{12}\right)\left[\left(z^{+}\right)^{2} t_{2}\left(t-t_{2}\right)-z^{+}(z+\delta)^{+} t_{1}\left(t-t_{1}\right)\right] \\
& \times\left\{\frac{1}{\left[\left(t-t_{2}\right)^{2}+z^{2}\right]^{3}}\left(\overleftarrow{\partial}_{z^{-}}-\vec{\partial}_{z^{-}}\right) \frac{1}{\left[\left(t-t_{1}\right)^{2}+z^{2}\right]^{3}}\right\} \\
& \times\left\{\frac{1}{\left(t_{1}^{2}+z^{2}\right)^{\frac{3}{2}}}\left(\overleftarrow{\partial}_{z^{-}}+\vec{\partial}_{\delta^{-}}\right) \frac{1}{|\delta|^{3}}\left(\overleftarrow{\partial}_{\delta^{-}}-\vec{\partial}_{z^{-}}\right) \frac{1}{\left[t_{2}^{2}+\left(z+\delta^{\prime}\right)^{2}\right]^{\frac{3}{2}}}\right\}_{\delta^{\prime}=\delta}
\end{aligned}
$$

In the small $\delta$ limit, the contribution is again dominated by regions $D_{1}$ and $D_{2}$ as in G.12). The contribution from $D_{2}$ is of the form $\frac{\left(\delta^{+}\right)^{6} \epsilon(t)}{\delta^{9} t^{6}}\left[1+\mathcal{O}\left(\frac{\delta^{2}}{t^{2}}\right)\right]$, and should cancel in the end just as in the $\langle j j T\rangle$ case. The contribution from $D_{1}$ is easily seen to vanish at order $\frac{\left(\delta^{+}\right)^{6}}{\delta^{8} t^{7}}$, and does not contribute to the one-loop coefficient of the parity odd $\langle T T T\rangle$ structure.

Diagram III is given by

$$
\begin{aligned}
\int d^{3} y_{1} d^{3} y_{2}\left\langle A_{i}\left(y_{1}\right) A_{j}\left(y_{2}\right)\right\rangle \operatorname{Tr}\left[\gamma^{j}\left\langle\psi\left(y_{1}\right) \bar{\psi}\left(x_{1}\right)\right\rangle \notin\left(\varepsilon \cdot \overleftrightarrow{\partial}_{x_{1}}\right)\left\langle\psi\left(x_{1}\right) \bar{\psi}\left(y_{2}\right)\right\rangle \gamma^{i}\left\langle\psi\left(y_{2}\right) \bar{\psi}\left(x_{2}\right)\right\rangle\right. \\
\left.\times \notin\left(\varepsilon \cdot \overleftrightarrow{\partial}_{x_{2}}\right)\left\langle\psi\left(x_{2}\right) \bar{\psi}\left(x_{3}\right)\right\rangle \notin\left(\varepsilon \cdot \overleftrightarrow{\partial}_{x_{3}}\right)\left\langle\psi\left(x_{3}\right) \bar{\psi}\left(y_{1}\right)\right\rangle\right]
\end{aligned}
$$


The loop integral is computed by

$$
\begin{aligned}
& \int d^{3} y_{1} d^{3} y_{2} \epsilon\left(y_{12}^{0}\right) \delta^{2}\left(\vec{y}_{12}\right) \epsilon_{i j} \operatorname{Tr}\left[\gamma^{i} y_{1} \gamma^{+}\left(-y_{2}\right) \gamma^{j}\left(y_{2}+\not\right) \gamma^{+}(-\not x-\not) \gamma^{+}\left(\not x-y_{1}\right)\right] \\
& \quad \times\left(\frac{1}{\left|y_{1}\right|^{3}} \overleftrightarrow{\partial}_{-} \frac{1}{\left|y_{2}\right|^{3}}\right)\left[\frac{1}{\left|x-y_{1}\right|^{3}} \overleftrightarrow{\partial}_{x^{-}} \frac{1}{|x+\delta|^{3}} \overleftrightarrow{\partial}_{\delta^{-}} \frac{1}{\left|\delta+y_{2}\right|^{3}}\right] \\
& =-16 i \delta^{+} \int d^{2} z d t_{1} d t_{2} \epsilon\left(t_{12}\right)\left[\left(z^{+}\right)^{2} t_{2}^{2}+z^{+}(z+\delta)^{+} t_{1}\left(t-t_{1}\right)\right]\left[\frac{1}{\left(t_{1}^{2}+z_{1}^{2}\right)^{\frac{3}{2}}} \overleftrightarrow{\partial}_{z^{-}} \frac{1}{\left(t_{2}^{2}+z^{2}\right)^{\frac{3}{2}}}\right] \\
& \quad \times\left\{\frac{1}{\left[\left(t-t_{1}\right)^{2}+z^{2}\right]^{\frac{3}{2}}}\left(\overleftarrow{\partial}_{z^{-}}+\vec{\partial}_{\delta^{-}}\right) \frac{1}{\left(t^{2}+\delta^{2}\right)^{\frac{3}{2}}}\left(-\overleftarrow{\partial}_{\delta^{-}}+\vec{\partial}_{z^{-}}\right) \frac{1}{\left[t_{2}^{2}+\left(z+\delta^{\prime}\right)^{2}\right]^{\frac{3}{2}}}\right\}_{\delta^{\prime}=\delta} .
\end{aligned}
$$

In the small $\delta$ limit, the contribution from region $D_{2}$ should cancel against diagrams I,II. We only need to compute the contribution from region $D_{1}$, given by

$$
\begin{aligned}
& \sim-16 i \delta^{+} \int d^{2} z d t_{1} d t_{2} \epsilon\left(t_{12}\right) z^{+}(z+\delta)^{+} t_{1} t\left[\frac{1}{\left(t_{1}^{2}+z^{2}\right)^{\frac{3}{2}}} \overleftrightarrow{\partial}_{z^{-}} \frac{1}{\left(t_{2}^{2}+z^{2}\right)^{\frac{3}{2}}}\right] \\
& \quad \times\left\{\frac{1}{\left(t^{2}+z^{2}\right)^{\frac{3}{2}}}\left(\overleftarrow{\partial}_{z^{-}}+\vec{\partial}_{\delta^{-}}\right) \frac{1}{\left(t^{2}+\delta^{2}\right)^{\frac{3}{2}}}\right\} \frac{-3(z+\delta)^{+}}{\left[t_{2}^{2}+(z+\delta)^{2}\right]^{\frac{5}{2}}} \\
& \sim-16 i \frac{\delta^{+}}{t^{7}} \int d^{2} z d t_{1} d t_{2} \epsilon\left(t_{12}\right) z^{+}(z+\delta)^{+} t_{1}\left[\frac{-3 z^{+}\left(t_{1}^{2}-t_{2}^{2}\right)}{\left(t_{1}^{2}+z^{2}\right)^{\frac{5}{2}}\left(t_{2}^{2}+z^{2}\right)^{\frac{5}{2}}}\right] \frac{9\left((z+\delta)^{+}\right)^{2}}{\left[t_{2}^{2}+(z+\delta)^{2}\right]^{\frac{5}{2}}} \\
& \sim 27 \cdot 16 i \frac{\delta^{+}}{t^{7}} \int d^{2} z d t_{1} d t_{2} \epsilon\left(t_{12}\right) \frac{\left(z^{+}\right)^{2}\left((z+\delta)^{+}\right)^{3} t_{1}\left(t_{1}^{2}-t_{2}^{2}\right)}{\left(t_{1}^{2}+z^{2}\right)^{\frac{5}{2}}\left(t_{2}^{2}+z^{2}\right)^{\frac{5}{2}}\left[t_{2}^{2}+(z+\delta)^{2}\right]^{\frac{5}{2}}} .
\end{aligned}
$$

Using

$$
\int d t_{1} \epsilon\left(t_{12}\right) \frac{t_{1}\left(t_{1}^{2}-t_{2}^{2}\right)}{\left(z^{2}+t_{1}^{2}\right)^{\frac{5}{2}}}=\frac{4}{3} \frac{1}{\left(z^{2}+t_{2}^{2}\right)^{\frac{1}{2}}},
$$

We can write the RHS of (G.27) in a Lorentz invariant form

$$
27 \cdot 16 i \frac{\delta^{+}}{t^{7}} \int d^{3} y \frac{\left(y^{+}\right)^{2}\left((y+\delta)^{+}\right)^{3}}{|y|^{6}|y+\delta|^{5}}=-9 \cdot 256 \pi i \frac{\left(\delta^{+}\right)^{6}}{\delta^{8} t^{7}} .
$$

This is the only nontrivial one-loop contribution to the parity odd $\langle T T T\rangle$ structure. Putting back in the normalization factors, we obtain

$$
\langle T T T\rangle_{1-\text { loop }} \longrightarrow-\frac{9 N \lambda}{2 \pi^{3}} \frac{\left(\delta^{+}\right)^{6}}{\delta^{8} t^{7}}
$$




\section{G.3 Two-loop parity preserving structure of the three point functions}

The goal of this section is to compute the (parity-preserving) two-loop contribution to three point functions $\langle J J J\rangle$. In particular, we will demonstrate that the "free scalar tensor structure" of $\langle J J J\rangle$ receives a nontrivial contribution at two-loop.

\section{G.3.1 $\langle T T T\rangle$ at two-loop}

Let us begin with three point function of the stress-energy tensor $\langle T T T\rangle . T_{\mu \nu}(x)$ is given by

$$
T_{\mu \nu}=\bar{\psi} \gamma_{(\mu} \overleftrightarrow{D}_{\nu)} \psi
$$

or when contracted with a null polarization vector $\varepsilon$,

$$
T_{\varepsilon}=\bar{\psi} \phi(\varepsilon \cdot \overleftrightarrow{D}) \psi
$$

The three point function $\left\langle T_{\varepsilon_{1}}\left(x_{1}\right) T_{\varepsilon_{2}}\left(x_{2}\right) T_{\varepsilon_{3}}\left(x_{3}\right)\right\rangle$ takes the form

$$
\left\langle T_{\varepsilon_{1}}\left(x_{1}\right) T_{\varepsilon_{2}}\left(x_{2}\right) T_{\varepsilon_{3}}\left(x_{3}\right)\right\rangle=\tilde{c}_{B} F_{222}^{B}\left(\left\{x_{i}, \varepsilon_{i}\right\}\right)+\tilde{c}_{F} F_{222}^{F}\left(\left\{x_{i}, \varepsilon_{i}\right\}\right)+\text { parity violating term. }
$$

where $F_{222}^{B}\left(\left\{x_{i}, \varepsilon_{i}\right\}\right)$ and $F_{222}^{F}\left(\left\{x_{i}, \varepsilon_{i}\right\}\right)$ are proportional to the three point function of stress-energy tensors for a free scalar and a free fermion, $\langle T T T\rangle_{B}$ and $\langle T T T\rangle_{F}$. Our normalization convention is

$$
\begin{aligned}
& F_{222}^{F}\left(\left\{x_{i}, \varepsilon_{i}\right\}\right)=\left.\frac{\sinh \left(\frac{Q_{1}+Q_{2}+Q_{3}}{2}\right) \sinh P_{1} \sinh P_{3} \sinh P_{3}}{\left|x_{12}\right|\left|x_{23}\right| x_{31} \mid}\right|_{\lambda_{1}^{4} \lambda_{2}^{4} \lambda_{3}^{4}}, \\
& F_{222}^{B}\left(\left\{x_{i}, \varepsilon_{i}\right\}\right)=\left.\frac{\cosh \left(\frac{Q_{1}+Q_{2}+Q_{3}}{2}\right) \cosh P_{1} \cosh P_{3} \cosh P_{3}}{\left|x_{12}\right|\left|x_{23}\right| x_{31} \mid}\right|_{\lambda_{1}^{4} \lambda_{2}^{4} \lambda_{3}^{4}} .
\end{aligned}
$$

The parity-violating term in G.33 has been described in previous subsections; it only receives contribution at odd loop order, and its one-loop contribution has been evaluated explicitly.

The constant coefficients $c_{B}$ and $c_{F}$ in G.33 have the following perturbative expansions in $\lambda$ in the planar limit:

$$
\tilde{c}_{B}=g_{0} N\left(c_{B}^{(2)} \lambda^{2}+\cdots\right), \quad \tilde{c}_{F}=g_{0} N\left(1+c_{F}^{(2)} \lambda^{2}+\cdots\right)
$$

where $g_{0}$ is an overall constant having to do with the normalization convention on the stress We will now compute the two loop contribution $c_{B}^{(2)}$. Since we are only interested 
in extracting the coefficient of $\langle T T T\rangle_{B}$ tensor structure, it suffices to consider a special configuration of $\left(x_{i}, \varepsilon_{i}\right)$ at which $\langle T T T\rangle_{F}$ vanishes and $\langle T T T\rangle_{B}$ simplifies.

The stress-energy tensors of a free complex scalar $\phi$ and a free complex fermion $\psi$ are

$$
T_{\varepsilon}^{B}=2|\varepsilon \cdot \partial \phi|^{2}-\frac{1}{4}(\varepsilon \cdot \partial)^{2}|\phi|^{2}, \quad T_{\varepsilon}^{F}=\bar{\psi} \ddagger \varepsilon \cdot \overleftrightarrow{\partial} \psi
$$

With this normalization, the three point functions are related to $F_{222}^{B}$ and $F_{222}^{F}$ G.34 by

$$
\begin{aligned}
\left\langle T_{\varepsilon_{1}}^{B}\left(x_{1}\right) T_{\varepsilon_{2}}^{B}\left(x_{2}\right) T_{\varepsilon_{3}}^{B}\left(x_{3}\right)\right\rangle & =-\frac{432}{\pi^{3}} F_{222}^{B}\left(\left\{x_{i}, \varepsilon_{i}\right\}\right), \\
\left\langle T_{\varepsilon_{1}}^{F}\left(x_{1}\right) T_{\varepsilon_{2}}^{F}\left(x_{2}\right) T_{\varepsilon_{3}}^{F}\left(x_{3}\right)\right\rangle & =-\frac{432}{\pi^{3}} F_{222}^{F}\left(\left\{x_{i}, \varepsilon_{i}\right\}\right) .
\end{aligned}
$$

Now, restrict to the special case $\varepsilon_{1}=\varepsilon_{2}=\varepsilon_{3}=\varepsilon$. Further, at the special configuration $x_{13} \cdot \varepsilon=0$ (the labeling of the three operators is an arbitrary choice), it can be seen that $\left\langle T_{\varepsilon}^{F} T_{\varepsilon}^{F} T_{\varepsilon}^{F}\right\rangle$ vanishes, while $\left\langle T_{\varepsilon}^{B} T_{\varepsilon}^{B} T_{\varepsilon}^{B}\right\rangle$ does not vanish.

To extract $c_{B}$, it suffices to take the limit $\vec{x}_{12}=\vec{\delta} \rightarrow 0$, while keeping $\vec{x}_{13}$ finite and subject to the condition $x_{13} \cdot \varepsilon=0$. In this limit, we have

$$
\left\langle T_{\varepsilon}^{B}\left(x_{1}\right) T_{\varepsilon}^{B}\left(x_{2}\right) T_{\varepsilon}^{B}\left(x_{3}\right)\right\rangle=-\frac{315}{32 \pi^{3}} \frac{(\delta \cdot \varepsilon)^{6}}{\left|x_{13}\right|^{6}|\delta|^{9}}, \quad\left\langle T_{\varepsilon}^{F}\left(x_{1}\right) T_{\varepsilon}^{F}\left(x_{2}\right) T_{\varepsilon}^{F}\left(x_{3}\right)\right\rangle=0 .
$$

We find it the simplest to work in temporal gauge $A_{0}=0$, when the CS cubic coupling disappears and the gauge field propagator is

$$
\left\langle A_{i}(x) A_{j}(0)\right\rangle=\frac{2 \pi i}{k} \epsilon_{i j} \operatorname{sign}\left(x^{0}\right) \delta^{2}(\vec{x})
$$

Without loss of generality, we will choose the temporal direction to be orthogonal to $\varepsilon$; in other words, $\varepsilon$ is assumed to be a purely spatial complex null vector. Note however we can not assume all of $x_{1,2,3}$ to be purely spatial. We can proceed by assuming that $x_{1}, x_{2}$ are purely spatial, while $x_{3}$ is a general vector. As we have seen in the two-loop fermion self-energy, there can be unphysical divergences in the temporal gauge, which are expected to drop out in gauge invariant correlators.

There are potentially many two-loop diagrams that could contribute, but most of them do not contribute to terms of order

$$
\frac{(\delta \cdot \varepsilon)^{6}}{\left|x_{13}\right|^{6}|\delta|^{9}}
$$

in the limit

$$
\varepsilon_{i}=\varepsilon, \quad \varepsilon^{2}=\varepsilon \cdot e_{0}=\varepsilon \cdot x_{13}=0, \quad \delta=x_{12} \rightarrow 0, \quad x_{13} \text { finite. }
$$


For instance, any diagram with a bare propagator $\left\langle\psi\left(x_{1}\right) \bar{\psi}\left(x_{3}\right)\right\rangle$ vanishes, because this propagator is inserted in a trace $\operatorname{Tr}\left[\notin\left\langle\psi\left(x_{1}\right) \bar{\psi}\left(x_{3}\right)\right\rangle \notin \cdots\right] \propto x_{12} \cdot \varepsilon \operatorname{Tr}[\notin \cdots]=0$. Slightly less obvious is that diagrams involving bare propagators $\left\langle\psi\left(x_{2}\right) \bar{\psi}\left(x_{1}\right)\right\rangle$ or $\left\langle\psi\left(x_{3}\right) \bar{\psi}\left(x_{2}\right)\right\rangle$, thought not identically zero, do not contribute to G.40 in the limit G.41 either. The former comes with at most two derives acting on $\varepsilon \cdot \delta /|\delta|^{3}$ from the structure $\operatorname{Tr}\left[\notin\left\langle\psi\left(x_{2}\right) \bar{\psi}\left(x_{1}\right)\right\rangle \notin \cdots\right]$, which at most goes like $|\delta|^{-7}$ in the small $\delta$ limit. The latter involves at least one derivative $\varepsilon \cdot \partial_{x_{3}}$ acting on the factor $\sim 1 /\left|x_{13}\right|^{6}$ from the denominators in the two fermion propagators connected to $x_{3}$; in this case there are no additional factors that scales like $x_{13}$ in the numerator, and so the result cannot contribute in the large $x_{13}$ limit at order $\mathcal{O}\left(\left|x_{13}\right|^{-6}\right)$ as in G.40). Further, if one of the fermion propagators between two $T$ vertices, $\left\langle\psi\left(x_{i}\right) \bar{\psi}\left(x_{j}\right)\right\rangle$, is corrected by a one-loop self-energy diagram, such a diagram vanishes in the configuration (G.41) because one encounters $\operatorname{Tr}(\notin \mathbb{I} \notin \cdots)$ in the trace over Gamma matrices. Therefore, the only diagrams we need to consider are of the form shown in figure 7 . In fact, there is only one such diagram that contributes in the limit G.41), namely the one with $T\left(x_{3}\right)$ being the vertex on the left, in figure 7 .

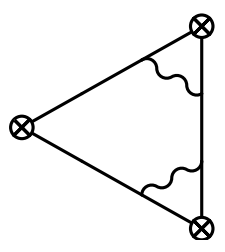

Figure 7

This diagram is computed as

$$
\begin{aligned}
- & \frac{1}{2}(2 \pi i \lambda)^{2} \int d^{3} y_{1} d^{3} y_{2} d^{3} z_{1} d^{3} z_{2} \prod_{i=1}^{2} \operatorname{sign}\left(y_{i}^{0}-z_{i}^{0}\right) \delta^{2}\left(\vec{y}_{i}-\vec{z}_{i}\right) \\
\times & \operatorname{Tr}\left[\left\langle\psi\left(z_{1}\right) \bar{\psi}\left(x_{3}\right)\right\rangle \notin \varepsilon \cdot \overleftrightarrow{\partial}_{x_{3}}\left\langle\psi\left(x_{3}\right) \bar{\psi}\left(z_{2}\right)\right\rangle \gamma_{k}\left\langle\psi\left(z_{2}\right) \bar{\psi}\left(x_{2}\right)\right\rangle \notin \varepsilon \cdot \overleftrightarrow{\partial}_{x_{2}}\left\langle\psi\left(x_{2}\right) \bar{\psi}\left(y_{2}\right)\right\rangle \gamma_{\ell} \epsilon^{k \ell}\right. \\
& \left.\cdot\left\langle\psi\left(y_{2}\right) \bar{\psi}\left(y_{1}\right)\right\rangle \gamma_{i}\left\langle\psi\left(y_{1}\right) \bar{\psi}\left(x_{1}\right)\right\rangle \notin \varepsilon \cdot \overleftrightarrow{\partial}_{x_{1}}\left\langle\psi\left(x_{1}\right) \bar{\psi}\left(z_{1}\right)\right\rangle \gamma_{j} \epsilon^{i j}\right]
\end{aligned}
$$

In the limit G.41, G.42 is given by the following loop integral multiplied by a normalization factor $-\frac{1}{2}(2 \pi i \lambda)^{2}(4 \pi)^{-7}$,

$$
\begin{aligned}
- & \frac{3}{\left|x_{13}\right|^{6}} \int d^{3} y_{1} d^{3} y_{2} d^{3} z_{1} d^{3} z_{2} \frac{\varepsilon \cdot z_{12}}{\left|y_{12}\right|^{3}} \prod_{i=1}^{2} \operatorname{sign}\left(y_{i}^{0}-z_{i}^{0}\right) \delta^{2}\left(\vec{y}_{i}-\vec{z}_{i}\right)\left[\frac{1}{\left|x_{i}-z_{i}\right|^{3}} \varepsilon \cdot \overleftrightarrow{\partial} x_{x_{i}} \frac{1}{\left|x_{i}-y_{i}\right|^{3}}\right] \\
& \times \operatorname{Tr}\left[\not \gamma_{k}\left(\not_{2}-\not \not_{2}\right) \notin\left(\not x_{2}-y_{2}\right) \gamma_{\ell} \epsilon^{k \ell} y_{21} \gamma_{i}\left(\not y_{1}-\not x_{1}\right) \notin\left(\not x_{1}-\not \not_{1}\right) \gamma_{j} \epsilon^{i j}\right]
\end{aligned}
$$


Using the assumption that $\varepsilon$ is purely spatial, we have $\gamma_{i} \notin \gamma_{j} \epsilon^{i j}=0$. The trace of product of Gamma matrices in the integrand can be simplified as as

$$
\begin{aligned}
& \operatorname{Tr}\left[\notin \gamma_{k}\left(\not_{2}-\not x_{2}\right) \notin\left(\not_{2}-y_{2}\right) \gamma_{\ell} \epsilon^{k \ell} y_{21} \gamma_{i}\left(y_{1}-\not x_{1}\right) \notin\left(\not x_{1}-\not x_{1}\right) \gamma_{j} \epsilon^{i j}\right] \\
& =\operatorname{Tr}\left[\notin\left(\not_{2}-\not x_{2}\right) \gamma_{k} \notin\left(\not \not_{2}-y_{2}\right) \gamma_{\ell} \epsilon^{k \ell} y_{21} \gamma_{i}\left(y_{1}-\not x_{1}\right) \notin \gamma_{j}\left(\not_{1}-\not k_{1}\right) \epsilon^{i j}\right] \\
& =4\left(x_{2}-y_{2}\right)_{\ell}\left(y_{1}-x_{1}\right)_{i} \operatorname{Tr}\left[\notin\left(\not_{2}-\not x_{2}\right) \gamma_{k} \notin \epsilon^{k \ell} y_{21} \notin \gamma_{j}\left(\not_{1}-\not k_{1}\right) \epsilon^{i j}\right] \\
& =4 \operatorname{Tr}\left[\notin\left(\not_{2}-\not \not_{2}\right) \gamma^{3}\left(\not x_{2}^{s}-y_{2}^{s}\right) \notin y_{21} \notin \gamma^{3}\left(y_{1}^{s}-\not x_{1}^{s}\right)\left(\not_{1}-\not k_{1}\right)\right]
\end{aligned}
$$

where $\not_{i}^{s}, y_{i}^{s}$ denotes the spatial part $\vec{x}_{i} \cdot \vec{\gamma}, \vec{y}_{i} \cdot \vec{\gamma}$. Define $\check{y}_{i}=\left(-y_{i}^{0}, \vec{y}_{i}\right)$, i.e. the vector with the time component flipped. Taking into account the restriction $z_{i}^{s}=y_{i}^{s}$ due to the delta functions in the temporal gauge propagators, we can write the above trace as

$$
\begin{aligned}
& 4 \operatorname{Tr}\left[\notin\left(z_{2}^{0} \gamma^{3}\right)\left(\not x_{2}^{s}-y_{2}^{s}\right) \check{y}_{21} \notin\left(y_{1}^{s}-\not x_{1}^{s}\right)\left(-z_{1}^{0} \gamma^{3}\right)\right] \\
& =4 z_{1}^{0} z_{2}^{0} \operatorname{Tr}\left[\notin\left(\not \not_{2}^{s}-y_{2}^{s}\right) \notin \check{y}_{21} \notin\left(y_{1}^{s}-\not x_{1}^{s}\right)\right] \\
& =32 z_{1}^{0} z_{2}^{0} \varepsilon \cdot y_{21} \varepsilon \cdot\left(x_{1}-y_{1}\right) \varepsilon \cdot\left(x_{2}-y_{2}\right) .
\end{aligned}
$$

The loop integral G.43 can be then written as

$$
\begin{aligned}
& -\frac{27 \cdot 32}{\left|x_{13}\right|^{6}} \int d^{3} y_{1} d^{3} y_{2} d^{3} z_{1} d^{3} z_{2} \frac{\left[\varepsilon \cdot y_{12} \varepsilon \cdot\left(x_{1}-y_{1}\right) \varepsilon \cdot\left(x_{2}-y_{2}\right)\right]^{2}}{\left|y_{12}\right|^{3}} \\
& \quad \times \prod_{i=1}^{2} \operatorname{sign}\left(y_{i}^{0}-z_{i}^{0}\right) \delta^{2}\left(\vec{y}_{i}-\vec{z}_{i}\right) \frac{z_{i}^{0}\left[\left(y_{i}^{0}\right)^{2}-\left(z_{i}^{0}\right)^{2}\right]}{\left|y_{i}-x_{i}\right|^{5}\left|z_{i}-x_{i}\right|^{5}}
\end{aligned}
$$

After performing the integral over $z_{1}^{0}, z_{2}^{0}$, which turn out to give very simple results, G.46) reduces to

$$
\begin{aligned}
- & \frac{3 \cdot 2^{9}}{\left|x_{13}\right|^{6}} \int d^{3} y_{1} d^{3} y_{2} \frac{\left[\varepsilon \cdot y_{12} \varepsilon \cdot\left(x_{1}-y_{1}\right) \varepsilon \cdot\left(x_{2}-y_{2}\right)\right]^{2}}{\left|y_{12}\right|^{3}} \frac{1}{\left|y_{1}-x_{1}\right|^{6}\left|y_{2}-x_{2}\right|^{6}} \\
=- & \frac{3 \cdot 2^{9}}{\left|x_{13}\right|^{6}} \frac{\Gamma\left(\frac{15}{2}\right)}{\Gamma(3)^{2} \Gamma\left(\frac{3}{2}\right)} \int_{0}^{1} d a \int_{0}^{1-a} d b a^{2} b^{2}(1-a-b)^{\frac{1}{2}} \\
& \times \int d^{3} y_{1} d^{3} y_{2} \frac{\left[\varepsilon \cdot\left(y_{12}+\delta\right) \varepsilon \cdot y_{1} \varepsilon \cdot y_{2}\right]^{2}}{\left[a y_{1}^{2}+b y_{2}^{2}+(1-a-b)\left(y_{12}+\delta\right)^{2}\right]^{\frac{15}{2}}} \\
=- & \frac{3 \cdot 2^{9}}{\left|x_{13}\right|^{6}} \frac{\Gamma\left(\frac{15}{2}\right)}{\Gamma(3)^{2} \Gamma\left(\frac{3}{2}\right)} \int_{0}^{1} d a \int_{0}^{1-a} d b a^{2} b^{2}(1-a-b)^{\frac{1}{2}} \\
& \times \int d^{3} y_{1} d^{3} y_{2} \frac{(u / a)^{2}(u / b)^{2}(1-u / a-u / b)^{2}(\varepsilon \cdot \delta)^{6}}{\left[a y_{1}^{2}+b y_{2}^{2}+(1-a-b) y_{12}^{2}+\delta^{2} u\right]^{\frac{15}{2}}}
\end{aligned}
$$

where in the last line we defined

$$
u \equiv \frac{a b(1-a-b)}{a(1-a)+b(1-b)-a b} .
$$


We can further shift $y_{2}$, and calculate the integral (G.47) as

$$
\begin{aligned}
&- \frac{3 \cdot 2^{9}}{\left|x_{13}\right|^{6}} \frac{\Gamma\left(\frac{15}{2}\right)}{\Gamma(3)^{2} \Gamma\left(\frac{3}{2}\right)} \int_{0}^{1} d a \int_{0}^{1-a} d b a^{2} b^{2}(1-a-b)^{\frac{1}{2}} \\
& \times \int d^{3} y_{1} d^{3} y_{2} \frac{(u / a)^{2}(u / b)^{2}(1-u / a-u / b)^{2}(\varepsilon \cdot \delta)^{6}}{\left[a y_{1}^{2}+b y_{2}^{2}+(1-a-b) y_{12}^{2}+\delta^{2} u\right]^{\frac{15}{2}}} \\
&=-\frac{3 \cdot 2^{9}(\varepsilon \cdot \delta)^{6}}{\left|x_{13}\right|^{6}|\delta|^{9}} \frac{\Gamma\left(\frac{15}{2}\right)}{\Gamma(3)^{2} \Gamma\left(\frac{3}{2}\right)} \frac{8 \pi^{3}}{1287} \int_{0}^{1} d a \int_{0}^{1-a} d b \frac{a^{2} b^{2}(1-a-b)^{\frac{1}{2}}(u / a)^{2}(u / b)^{2}(1-u / a-u / b)^{2}}{u^{\frac{9}{2}}\left[\frac{a b(1-a-b)}{u}\right]^{\frac{3}{2}}} \\
&=- \frac{3 \cdot 2^{9}(\varepsilon \cdot \delta)^{6}}{\left|x_{13}\right|^{6}|\delta|^{9}} \frac{\Gamma\left(\frac{15}{2}\right)}{\Gamma(3)^{2} \Gamma\left(\frac{3}{2}\right)} \frac{8 \pi^{3}}{1287} \int_{0}^{1} d a \int_{0}^{1-a} d b \frac{a^{\frac{3}{2}}}{[a(1-a)+b(1-b)-a b]^{3}} \\
&=-2520 \pi^{4} \frac{(\varepsilon \cdot \delta)^{6}}{\left|x_{13}\right|^{6}|\delta|^{9}} .
\end{aligned}
$$

Putting back in the normalization factors, we found

$$
\begin{aligned}
& \left\langle T_{\varepsilon}\left(x_{1}\right) T_{\varepsilon}\left(x_{2}\right) T_{\varepsilon}\left(x_{3}\right)\right\rangle_{2-l o o p} \rightarrow-\frac{315 N \lambda^{2}}{2^{10} \pi} \frac{(\varepsilon \cdot \delta)^{6}}{\left|x_{13}\right|^{6}|\delta|^{9}} . \\
& \quad=N \frac{\pi^{2} \lambda^{2}}{32}\left\langle T_{\varepsilon}^{B}\left(x_{1}\right) T_{\varepsilon}^{B}\left(x_{2}\right) T_{\varepsilon}^{B}\left(x_{3}\right)\right\rangle_{x_{13} \cdot \varepsilon=0, x_{12}=\delta \rightarrow 0}
\end{aligned}
$$

This determines the two-loop coefficient $c_{B}^{(2)}$ in $\mathrm{G} .35$

$$
c_{B}^{(2)}=\frac{\pi^{2}}{32}
$$

\section{G.3.2 General spins}

Now let us consider the two-loop contribution to the three point function of higher spin currents, of the form $\left\langle J_{s_{1}} J_{s_{2}} J_{s_{3}}\right\rangle$. Once again we will take all $\varepsilon_{i}$ 's equal to $\varepsilon$, restrict to the case $\varepsilon \cdot x_{13}=0$, and take the limit $x_{12}=\delta \rightarrow 0$. As before, the free fermion $\langle J J J\rangle$ tensor structure vanishes in this case, and we can extract the coefficient of the free scalar $\langle J J J\rangle$ tensor structure by taking the limit G.41). More precisely, we expect

$$
\left\langle J_{s_{1}}\left(x_{1} ; \varepsilon\right) J_{s_{2}}\left(x_{2} ; \varepsilon\right) J_{s_{3}}\left(x_{3} ; \varepsilon\right)\right\rangle \rightarrow C_{s_{1} s_{2} s_{3}}^{B} \frac{(\varepsilon \cdot \delta)^{s_{1}+s_{2}+s_{3}}}{\left|x_{13}\right|^{2 s_{3}+2}|\delta|^{2 s_{1}+2 s_{2}+1}}
$$

in the limit of $\mathrm{G.41}$, and shall now determine the coefficients $C_{s_{1} s_{2} s_{3}}^{B}$. A generating function for the higher spin currents in CS-fermion theory (with a null polarization vector $\varepsilon$ ) is

$$
J(\varepsilon)=\bar{\psi} \notin f(\varepsilon \cdot \overleftarrow{D}, \varepsilon \cdot \vec{D}) \psi
$$


where

$$
f(u, v)=e^{u-v} \frac{\sin (2 \sqrt{u v})}{2 \sqrt{u v}} .
$$

Define $f_{n}(u, v)$ to be the part of $f(u, v)$ with total degree $n$ in $(u, v)$.

As in the discussion for the correlator of stress-energy tensor, the only two-loop diagram that contributes to $C_{s_{1} s_{2} s_{3}}^{B}$ in the limit (G.41) will be the one with two gluon lines connecting fermion propagators adjacent to $J_{s_{1}}$ and $J_{s_{2}}$. We can then effectively replace $D_{\mu}$ by $\partial_{\mu}$ in the current insertions. The loop integral associated with the diagram in figure 7 is

$$
\begin{aligned}
& \int d^{3} y_{1} d^{3} y_{2} d^{3} z_{1} d^{3} z_{2} \prod_{i=1}^{2} \operatorname{sign}\left(y_{i}^{0}-z_{i}^{0}\right) \delta^{2}\left(\vec{y}_{i}-\vec{z}_{i}\right) \\
& \times \operatorname{Tr}\left[\left\langle\psi\left(z_{1}\right) \bar{\psi}\left(x_{3}\right)\right\rangle \notin f_{s_{3}-1}\left(\varepsilon \cdot \overleftarrow{\partial}_{x_{3}}, \varepsilon \cdot \vec{\partial}_{x_{3}}\right)\left\langle\psi\left(x_{3}\right) \bar{\psi}\left(z_{2}\right)\right\rangle \gamma_{k}\left\langle\psi\left(z_{2}\right) \bar{\psi}\left(x_{2}\right)\right\rangle \notin f_{s_{2}-1}\left(\varepsilon \cdot \overleftarrow{\partial}_{x_{2}}, \varepsilon \cdot \vec{\partial}_{x_{2}}\right)\right. \\
& \left.\cdot\left\langle\psi\left(x_{2}\right) \bar{\psi}\left(y_{2}\right)\right\rangle \gamma_{\ell} \epsilon^{k \ell}\left\langle\psi\left(y_{2}\right) \bar{\psi}\left(y_{1}\right)\right\rangle \gamma_{i}\left\langle\psi\left(y_{1}\right) \bar{\psi}\left(x_{1}\right)\right\rangle \notin f_{s_{1}-1}\left(\varepsilon \cdot \overleftarrow{\partial}_{x_{1}}, \varepsilon \cdot \vec{\partial}_{x_{1}}\right)\left\langle\psi\left(x_{1}\right) \bar{\psi}\left(z_{1}\right)\right\rangle \gamma_{j} \epsilon^{i j}\right]
\end{aligned}
$$

Using the following relation involving the generating function $f(u, v)$,

$$
\begin{aligned}
& \frac{1}{|x-z|^{3}} f\left(\varepsilon \cdot \overleftarrow{\partial}_{x}, \varepsilon \cdot \vec{\partial}_{x}\right) \frac{1}{|x-y|^{3}} \\
& =\frac{1}{|x-z+\varepsilon|^{3}} \sum_{n=0}^{\infty} \frac{(-)^{n} 4^{n}}{(2 n+1) !}\left(\varepsilon \cdot \overleftarrow{\partial}_{x}\right)^{n}\left(\varepsilon \cdot \vec{\partial}_{x}\right)^{n} \frac{1}{|x-y-\varepsilon|^{3}} \\
& =\sum_{n=0}^{\infty} \frac{(-)^{n} 4^{n}}{(2 n+1) !}\left[\frac{(2 n+1) !}{2^{n} n !}\right]^{2} \frac{(\varepsilon \cdot(x-z))^{n}}{|x-z+\varepsilon|^{3+2 n}} \frac{(\varepsilon \cdot(x-y))^{n}}{|x-y-\varepsilon|^{3+2 n}} \\
& =\frac{1}{|x-y-\varepsilon|^{3}|x-z+\varepsilon|^{3}}\left[1+\frac{4 \varepsilon \cdot(x-y) \varepsilon \cdot(x-z)}{|x-y-\varepsilon|^{2}|x-z+\varepsilon|^{2}}\right]^{-\frac{3}{2}}
\end{aligned}
$$

we have

$$
\frac{1}{|x-z|^{3}} f_{s-1}\left(\varepsilon \cdot \overleftarrow{\partial}_{x}, \varepsilon \cdot \vec{\partial}_{x}\right) \frac{1}{|x-y|^{3}}=2^{s-1}\left(\begin{array}{c}
-\frac{3}{2} \\
s-1
\end{array}\right) \frac{1}{|x-z|^{3}|x-y|^{3}}\left[\frac{\varepsilon \cdot(x-z)}{|x-z|^{2}}-\frac{\varepsilon \cdot(x-y)}{|x-y|^{2}}\right]^{s-1}
$$

and in particular

$$
\begin{aligned}
& \frac{1}{\left|x_{3}-z_{1}\right|^{3}} f_{s_{3}-1}\left(\varepsilon \cdot \overleftarrow{\partial}_{x_{3}}, \varepsilon \cdot \vec{\partial}_{x_{3}}\right) \frac{1}{\left|x_{3}-z_{2}\right|^{3}} \rightarrow 2^{s_{3}-1}\left(\begin{array}{c}
-\frac{3}{2} \\
s_{3}-1
\end{array}\right) \frac{\left(\varepsilon \cdot z_{21}\right)^{s_{3}-1}}{|x|^{2 s_{3}+4}}, \quad\left|x_{3}\right| \gg\left|z_{1}\right|,\left|z_{2}\right| ; \\
& \frac{1}{\left|x_{i}-z_{i}\right|^{3}} f_{s_{i}-1}\left(\varepsilon \cdot \overleftarrow{\partial}_{x_{i}}, \varepsilon \cdot \vec{\partial}_{x_{i}}\right) \frac{1}{\left|x_{i}-y_{i}\right|^{3}} \\
& =2^{s_{i}-1}\left(\begin{array}{c}
-\frac{3}{2} \\
s_{i}-1
\end{array}\right) \frac{\left(\varepsilon \cdot\left(x_{i}-y_{i}\right)\right)^{s_{i}-1}\left[\left(y_{i}^{0}\right)^{2}-\left(z_{i}^{0}\right)^{2}\right]^{s_{i}-1}}{\left|x_{i}-z_{i}\right|^{2 s_{i}+1}\left|x_{i}-y_{i}\right|^{2 s_{i}+1}}, \quad\left(\vec{y}_{i}=\vec{z}_{i}\right) .
\end{aligned}
$$


The integral G.55) then reduces to

$$
\begin{aligned}
& \frac{\mathcal{N}_{s_{1}} \mathcal{N}_{s_{2}} \mathcal{N}_{s_{3}}}{\left|x_{13}\right|^{2 s_{3}+2}} \int d^{3} y_{1} d^{3} y_{2} d^{3} z_{1} d^{3} z_{2} \frac{\left(\varepsilon \cdot z_{21}\right)^{s_{3}-1}}{\left|y_{12}\right|^{3}} \\
& \quad \times \prod_{i=1}^{2} \operatorname{sign}\left(y_{i}^{0}-z_{i}^{0}\right) \delta^{2}\left(\vec{y}_{i}-\vec{z}_{i}\right) \frac{\left(\varepsilon \cdot\left(x_{i}-y_{i}\right)\right)^{s_{i}-1}\left[\left(y_{i}^{0}\right)^{2}-\left(z_{i}^{0}\right)^{2}\right]^{s_{i}-1}}{\left|x_{i}-z_{i}\right|^{2 s_{i}+1}\left|x_{i}-y_{i}\right|^{2 s_{i}+1}} \\
& \quad \times \operatorname{Tr}\left[\notin\left(\not z_{2}-\not x_{2}\right) \gamma_{k} \notin\left(\not x_{2}-y_{2}\right) \gamma_{\ell} \epsilon^{k \ell} y_{21} \gamma_{i}\left(y_{1}-\not x_{1}\right) \notin \gamma_{j}\left(\not x_{1}-\not x_{1}\right) \epsilon^{i j}\right] \\
& =(-)^{s_{3}-1} \frac{32 \mathcal{N}_{s_{1}} \mathcal{N}_{s_{2}} \mathcal{N}_{s_{3}}}{\left|x_{13}\right|^{2 s_{3}+2}} \int d^{3} y_{1} d^{3} y_{2} d^{3} z_{1} d^{3} z_{2} \frac{\left(\varepsilon \cdot y_{12}\right)^{s_{3}}\left(\varepsilon \cdot\left(x_{1}-y_{1}\right)\right)^{s_{1}}\left(\varepsilon \cdot\left(x_{2}-y_{2}\right)\right)^{s_{2}}}{\left|y_{12}\right|^{3}} \\
& \quad \times \prod_{i=1}^{2} \operatorname{sign}\left(y_{i}^{0}-z_{i}^{0}\right) \delta^{2}\left(\vec{y}_{i}-\vec{z}_{i}\right) \frac{z_{i}^{0}\left[\left(y_{i}^{0}\right)^{2}-\left(z_{i}^{0}\right)^{2}\right]^{s_{i}-1}}{\left|x_{i}-z_{i}\right|^{2 s_{i}+1}\left|x_{i}-y_{i}\right|^{2 s_{i}+1}}
\end{aligned}
$$

where

$$
\mathcal{N}_{s} \equiv 2^{s-1}\left(\begin{array}{c}
-\frac{3}{2} \\
s-1
\end{array}\right) .
$$

Integrating out $z_{i}^{0}$ in G.59 now gives

$$
\begin{aligned}
(- & )^{s_{1}+s_{2}+s_{3}+1} \frac{32 \pi \Gamma\left(s_{1}\right) \Gamma\left(s_{2}\right) \mathcal{N}_{s_{1}} \mathcal{N}_{s_{2}} \mathcal{N}_{s_{3}}}{\Gamma\left(s_{1}+\frac{1}{2}\right) \Gamma\left(s_{2}+\frac{1}{2}\right)\left|x_{13}\right|^{2 s_{3}+2}} \int d^{3} y_{1} d^{3} y_{2} \frac{\left(\varepsilon \cdot y_{12}\right)^{s_{3}}\left(\varepsilon \cdot\left(x_{1}-y_{1}\right)\right)^{s_{1}}\left(\varepsilon \cdot\left(x_{2}-y_{2}\right)\right)^{s_{2}}}{\left|y_{12}\right|^{3}\left|x_{1}-y_{1}\right|^{2 s_{1}+2}\left|x_{2}-y_{2}\right|^{2 s_{2}+2}} \\
= & (-)^{s_{1}+s_{2}+s_{3}+1} \frac{32 \pi \mathcal{N}_{s_{1}} \mathcal{N}_{s_{2}} \mathcal{N}_{s_{3}}}{\Gamma\left(s_{1}+\frac{1}{2}\right) \Gamma\left(s_{2}+\frac{1}{2}\right)\left|x_{13}\right|^{2 s_{3}+2}} \frac{\Gamma\left(s_{1}+s_{2}+\frac{7}{2}\right)}{\Gamma\left(\frac{3}{2}\right) s_{1} s_{2}} \int_{0}^{1} d a \int_{0}^{1-a} d b a^{s_{1}} b^{s_{2}}(1-a-b)^{\frac{1}{2}} \\
& \times \int d^{3} y_{1} d^{3} y_{2} \frac{\left(\varepsilon \cdot\left(y_{12}+\delta\right)\right)^{s_{3}}\left(\varepsilon \cdot y_{1}\right)^{s_{1}}\left(\varepsilon \cdot y_{2}\right)^{s_{2}}}{\left[a y_{1}^{2}+b y_{2}^{2}+(1-a-b)\left(y_{12}+\delta\right)^{2}\right]^{s_{1}+s_{2}+\frac{7}{2}}} \\
= & (-)^{s_{1}+s_{2}+s_{3}+1} \frac{32 \pi \mathcal{N}_{s_{1}} \mathcal{N}_{s_{2}} \mathcal{N}_{s_{3}}}{\Gamma\left(s_{1}+\frac{1}{2}\right) \Gamma\left(s_{2}+\frac{1}{2}\right)\left|x_{13}\right|^{2 s_{3}+2} \frac{\Gamma\left(s_{1}+s_{2}+\frac{7}{2}\right)}{\Gamma\left(\frac{3}{2}\right) s_{1} s_{2}}} \int_{0}^{1} d a \int_{0}^{1-a} d b a^{s_{1}} b^{s_{2}}(1-a-b)^{\frac{1}{2}} \\
& \times \int d^{3} y_{1} d^{3} y_{2} \frac{(u / a)^{s_{1}}(u / b)^{s_{2}}(1-u / a-u / b)^{s_{3}}(\varepsilon \cdot \delta)^{s_{1}+s_{2}+s_{3}}}{\left[a y_{1}^{2}+b y_{2}^{2}+(1-a-b) y_{12}^{2}+\delta^{2} u\right]^{s_{1}+s_{2}+\frac{7}{2}}}
\end{aligned}
$$


Putting back in the normalization factors, we obtain

$$
\begin{aligned}
C_{s_{1} s_{2} s_{3}}^{B}= & -\frac{N(2 \pi i \lambda)^{2}}{2(4 \pi)^{7}}(-)^{s_{1}+s_{2}+s_{3}+1} \frac{32 \pi \mathcal{N}_{s_{1}} \mathcal{N}_{s_{2}} \mathcal{N}_{s_{3}}}{\Gamma\left(s_{1}+\frac{1}{2}\right) \Gamma\left(s_{2}+\frac{1}{2}\right)} \frac{\Gamma\left(s_{1}+s_{2}+\frac{7}{2}\right)}{\Gamma\left(\frac{3}{2}\right) s_{1} s_{2}} \\
& \times \int_{0}^{1} d a \int_{0}^{1-a} d b a^{s_{1}} b^{s_{2}}(1-a-b)^{\frac{1}{2}} \int d^{3} y_{1} d^{3} y_{2} \frac{(u / a)^{s_{1}}(u / b)^{s_{2}}(1-u / a-u / b)^{s_{3}}}{\left[a y_{1}^{2}+b y_{2}^{2}+(1-a-b) y_{12}^{2}+u\right]^{s_{1}+s_{2}+\frac{7}{2}}} \\
= & \frac{N \lambda^{2}}{2^{13} \pi^{5}}(-)^{s_{1}+s_{2}+s_{3}+1} \frac{32 \pi^{4} \mathcal{N}_{s_{1}} \mathcal{N}_{s_{2}} \mathcal{N}_{s_{3}}}{\Gamma\left(s_{1}+\frac{1}{2}\right) \Gamma\left(s_{2}+\frac{1}{2}\right)} \frac{\Gamma\left(s_{1}+s_{2}+\frac{1}{2}\right)}{\Gamma\left(\frac{3}{2}\right) s_{1} s_{2}} \\
& \times \int_{0}^{1} d a \int_{0}^{1-a} d b a^{s_{1}} b^{s_{2}}(1-a-b)^{\frac{1}{2}} \frac{(u / a)^{s_{1}}(u / b)^{s_{2}}(1-u / a-u / b)^{s_{3}}}{u^{s_{1}+s_{2}+\frac{1}{2}}\left[\frac{a b(1-a-b)}{u}\right]^{\frac{3}{2}}} \\
= & \frac{N \lambda^{2}}{2^{13} \pi^{5}}(-)^{s_{1}+s_{2}} \frac{32 \pi^{4} \mathcal{N}_{s_{1}} \mathcal{N}_{s_{2}} \mathcal{N}_{s_{3}}}{\Gamma\left(s_{1}+\frac{1}{2}\right) \Gamma\left(s_{2}+\frac{1}{2}\right)} \frac{\Gamma\left(s_{1}+s_{2}+\frac{1}{2}\right)}{\Gamma\left(\frac{3}{2}\right) s_{1} s_{2}} \\
& \times \int_{0}^{1} d a \int_{0}^{1-a} d b \frac{u}{1-a-b}(a b)^{-\frac{3}{2}}(1-u / a-u / b)^{s_{3}} \\
= & \frac{N \lambda^{2}}{2^{13} \pi^{5}}(-)^{s_{1}+s_{2}+s_{3}+1} \frac{32 \pi^{5} \mathcal{N}_{s_{1}} \mathcal{N}_{s_{2}} \mathcal{N}_{s_{3}}}{\Gamma\left(s_{1}+\frac{1}{2}\right) \Gamma\left(s_{2}+\frac{1}{2}\right)} \frac{\Gamma\left(s_{1}+s_{2}+\frac{1}{2}\right)}{\Gamma\left(\frac{3}{2}\right) s_{1} s_{2} s_{3}} \\
= & \frac{N \lambda^{2}}{2^{7} \pi^{2}} 2^{s_{1}+s_{2}+s_{3}} \Gamma\left(s_{3}+\frac{1}{2}\right) \frac{\Gamma\left(s_{1}+s_{2}+\frac{1}{2}\right)}{s_{1} ! s_{2} ! s_{3} !} .
\end{aligned}
$$

Our normalization for the higher spin currents is such that the two point functions of currents for a single free complex scalar and a free complex fermion are given by

$$
\frac{1}{4}\left\langle J_{s}^{B}(x, \varepsilon) J_{s}^{B}(0, \varepsilon)\right\rangle=\left\langle J_{s}^{F}(x, \varepsilon) J_{s}^{F}(0, \varepsilon)\right\rangle=\frac{1}{(4 \pi)^{2}} \frac{2^{4 s-1} \pi^{-\frac{1}{2}} \Gamma\left(s+\frac{1}{2}\right)}{s !} \frac{(\varepsilon \cdot x)^{2 s}}{|x|^{4 s+2}} .
$$

With this convention, the three point function coefficient of the currents for a free complex scalar in the limit of (G.52) is 31]

$$
C_{s_{1} s_{2} s_{3}}^{\text {free scalar }}=\frac{2^{s_{1}+s_{2}+s_{3}}}{4 \pi^{4}} \frac{\Gamma\left(s_{1}+s_{2}+\frac{1}{2}\right) \Gamma\left(s_{3}+\frac{1}{2}\right)}{\left(s_{1}\right) !\left(s_{2}\right) !\left(s_{3}\right) !}
$$

We see that $C_{s_{1} s_{2} s_{3}}^{B}$ is $C_{s_{1} s_{2} s_{3}}^{\text {free scalar }}$ times an $s_{i}$-independent constant. This constant has been computed in the previous subsection in the spin-2 case.

\section{References}

[1] S. Deser, R. Jackiw and S. Templeton, Phys. Rev. Lett. 48, 975 (1982).

[2] S. Deser, R. Jackiw and S. Templeton, Annals Phys. 140, 372 (1982) [Erratum-ibid. 185, 406 (1988)] [Annals Phys. 185, 406 (1988)] [Annals Phys. 281, 409 (2000)]. 
[3] E. Witten, "Quantum Field Theory and the Jones Polynomial," Commun. Math. Phys. 121, 351 (1989).

[4] G. W. Moore, N. Seiberg, "Lectures On Rcft,"

[5] J. Frohlich, T. Kerler, "Universality in quantum Hall systems," Nucl. Phys. B354, 369-417 (1991); J. Frohlich, A. Zee, "Large scale physics of the quantum Hall fluid," Nucl. Phys. B364, 517-540 (1991).

[6] E. A. Ivanov, "Chern-Simons matter systems with manifest N=2 supersymmetry," Phys. Lett. B268, 203-208 (1991); L. V. Avdeev, G. V. Grigorev, D. I. Kazakov, "Renormalizations in Abelian Chern-Simons field theories with matter," Nucl. Phys. B382, 561-580 (1992); L. V. Avdeev, D. I. Kazakov, I. N. Kondrashuk, "Renormalizations in supersymmetric and nonsupersymmetric nonAbelian Chern-Simons field theories with matter," Nucl. Phys. B391, 333-357 (1993); J. H. Schwarz, "Superconformal Chern-Simons theories," JHEP 0411, 078 (2004). hep-th/0411077); K. Hosomichi, K. -M. Lee, S. Lee, S. Lee, J. Park, "N=4 Superconformal ChernSimons Theories with Hyper and Twisted Hyper Multiplets," JHEP 0807, 091 (2008). arXiv:0805.3662 [hep-th]]. D. Gaiotto, E. Witten, "Janus Configurations, Chern-Simons Couplings, And The theta-Angle in N=4 Super Yang-Mills Theory," JHEP 1006, 097 (2010). arXiv:0804.2907 [hep-th]].

[7] D. Gaiotto, X. Yin, "Notes on superconformal Chern-Simons-Matter theories," JHEP 0708, 056 (2007). arXiv:0704.3740 [hep-th]].

[8] O. Aharony, O. Bergman, D. L. Jafferis, J. Maldacena, " $\mathrm{N}=6$ superconformal Chern-Simons-matter theories, M2-branes and their gravity duals," JHEP 0810, 091 (2008). arXiv:0806.1218 [hep-th]].

[9] J. M. Maldacena, "The large N limit of superconformal field theories and supergravity," Adv. Theor. Math. Phys. 2, 231 (1998) [Int. J. Theor. Phys. 38, 1113 (1999)] arXiv:hep-th/9711200; S. S. Gubser, I. R. Klebanov and A. M. Polyakov, "Gauge theory correlators from non-critical string theory," Phys. Lett. B 428, 105 (1998) arXiv:hep-th/9802109]; E. Witten, "Anti-de Sitter space and holography," Adv. Theor. Math. Phys. 2, 253 (1998) arXiv:hep-th/9802150.

[10] M. Benna, I. Klebanov, T. Klose, M. Smedback, "Superconformal ChernSimons Theories and AdS(4)/CFT(3) Correspondence," JHEP 0809, 072 (2008). arXiv:0806.1519 [hep-th]].

[11] K. Hosomichi, K. -M. Lee, S. Lee, S. Lee, J. Park, "N=5,6 Superconformal Chern-Simons Theories and M2-branes on Orbifolds," JHEP 0809, 002 (2008). arXiv:0806.4977[hep-th]].

[12] O. Aharony, O. Bergman, D. L. Jafferis, "Fractional M2-branes," JHEP 0811, 043 (2008). arXiv:0807.4924 [hep-th]]. 
[13] G. 't Hooft, "A Two-Dimensional Model for Mesons," Nucl. Phys. B75, 461 (1974).

[14] M. R. Gaberdiel, R. Gopakumar, "An $A d S_{3}$ Dual for Minimal Model CFTs," Phys. Rev. D83, 066007 (2011). [arXiv:1011.2986 [hep-th]].

[15] M. R. Gaberdiel, T. Hartman, "Symmetries of Holographic Minimal Models," JHEP 1105, 031 (2011). [arXiv:1101.2910 [hep-th]].

[16] C. Ahn, "The Large N 't Hooft Limit of Coset Minimal Models," arXiv:1106.0351 [hep-th]].

[17] M. R. Gaberdiel, R. Gopakumar, T. Hartman, S. Raju, "Partition Functions of Holographic Minimal Models," JHEP 1108, 077 (2011). [arXiv:1106.1897 [hep-th]].

[18] C. -M. Chang, X. Yin, "Higher Spin Gravity with Matter in $A d S_{3}$ and Its CFT Dual," arXiv:1106.2580 [hep-th]].

[19] K. Papadodimas, S. Raju, "Correlation Functions in Holographic Minimal Models," arXiv:1108.3077 [hep-th]].

[20] W. Siegel, "Supersymmetric Dimensional Regularization via Dimensional Reduction," Phys. Lett. B84, 193 (1979); W. Siegel, "Inconsistency of Supersymmetric Dimensional Regularization," Phys. Lett. B94, 37 (1980); L. V. Avdeev, A. A. Vladimirov, "Dimensional Regularization And Supersymmetry," Nucl. Phys. B219, 262 (1983).

[21] W. Chen, G. W. Semenoff, Y. -S. Wu, "Two loop analysis of nonAbelian ChernSimons theory," Phys. Rev. D46, $5521-5539$ (1992). hep-th/9209005.

[22] S. Sachdev, "Quantum Phase Transitions, " Cambridge University Press.

[23] P. Nozierres, "Theory of Interacting Fermi Systems, " Addison Wesley Reading Press.

[24] M. A. Vasiliev, "More On Equations Of Motion For Interacting Massless Fields Of All Spins In (3+1)-Dimensions," Phys. Lett. B 285, 225 (1992); M. A. Vasiliev, "Higher-spin gauge theories in four, three and two dimensions," Int. J. Mod. Phys. D 5, 763 (1996) arXiv:hep-th/9611024]; M. A. Vasiliev, "Higher spin gauge theories: Star-product and AdS space," arXiv:hep-th/9910096; M. A. Vasiliev, "Nonlinear equations for symmetric massless higher spin fields in (A)dS(d)," Phys. Lett. B 567, 139 (2003) arXiv:hep-th/0304049.

[25] I. R. Klebanov and A. M. Polyakov, "AdS dual of the critical O(N) vector model," Phys. Lett. B 550, 213 (2002) arXiv:hep-th/0210114.

[26] E. Sezgin and P. Sundell, "Massless higher spins and holography," Nucl. Phys. B 644, 303 (2002) [Erratum-ibid. B 660, 403 (2003)] arXiv:hep-th/0205131. 
[27] E. Sezgin, P. Sundell, "Holography in 4D (super) higher spin theories and a test via cubic scalar couplings," JHEP 0507, 044 (2005). hep-th/0305040].

[28] R. G. Leigh, A. C. Petkou, "Holography of the $\mathrm{N}=1$ higher spin theory on AdS(4)," JHEP 0306, 011 (2003). hep-th/0304217.

[29] D. J. Gross, E. Witten, "Possible Third Order Phase Transition in the Large N Lattice Gauge Theory," Phys. Rev. D21, 446-453 (1980).

[30] S. R. Wadia, "N = infinity Phase Transition in a Class of Exactly Soluble Model Lattice Gauge Theories," Phys. Lett. B93, 403 (1980).

[31] S. Giombi and X. Yin, "Higher Spin Gauge Theory and Holography: The ThreePoint Functions," arXiv:0912.3462 [hep-th].

[32] S. Giombi and X. Yin, "Higher Spins in AdS and Twistorial Holography," arXiv:1004.3736 [hep-th].

[33] S. Giombi, X. Yin, "On Higher Spin Gauge Theory and the Critical O(N) Model," arXiv:1105.4011 [hep-th]].

[34] H. Osborn and A. C. Petkou, "Implications of Conformal Invariance in Field Theories for General Dimensions," Annals Phys. 231, 311 (1994) arXiv:hep-th/9307010].

[35] J. M. Maldacena, G. L. Pimentel, "On graviton non-Gaussianities during inflation," JHEP 1109, 045 (2011). [arXiv:1104.2846 [hep-th]].

[36] S. Giombi, S. Prakash, X. Yin, "A Note on CFT Correlators in Three Dimensions," arXiv:1104.4317 [hep-th]].

[37] O. Aharony, G. Gur-Ari, R. Yacoby, "d=3 Vector Models Coupled to ChernSimons Gauge Theories," to appear.

[38] M. Smedback, "On thermodynamics of $\mathrm{N}=6$ superconformal Chern-Simons theory," JHEP 1004, 002 (2010). arXiv:1002.0841 [hep-th]].

[39] S. H. Shenker, X. Yin, "Vector Models in the Singlet Sector at Finite Temperature," arXiv:1109.3519 [hep-th]].

[40] O. Aharony, J. Marsano, S. Minwalla, K. Papadodimas and M. Van Raamsdonk, "The Hagedorn - deconfinement phase transition in weakly coupled large N gauge theories," Adv. Theor. Math. Phys. 8, 603 (2004) arXiv:hep-th/0310285.

[41] B. Sundborg, "The Hagedorn transition, deconfinement and N=4 SYM theory," Nucl. Phys. B573 (2000) 349-363. hep-th/9908001.

[42] J. Frohlich, C. King, "The Chern-simons Theory And Knot Polynomials," Commun. Math. Phys. 126, 167 (1989).

[43] J. M. F. Labastida, E. Perez, "Kontsevich integral for Vassiliev invariants from Chern-Simons perturbation theory in the light cone gauge," J. Math. Phys. 39, 5183-5198 (1998). hep-th/9710176. 
[44] H. Yukawa, "Quantum Theory of Nonlocal Fields. 1. Free Fields," Phys. Rev. 77, 219-226 (1950); "Quantum Theory Of Nonlocal Fields. 2: Irreducible Fields And Their Interaction," Phys. Rev. 80, 1047-1052 (1950).

[45] C. Itzykson, J. B. Zuber, "The Planar Approximation. 2.," J. Math. Phys. 21, 411 (1980).

[46] S. R. Wadia, "On The Dyson-schwinger Equations Approach To The Large N Limit: Model Systems And String Representation Of Yang-mills Theory," Phys. Rev. D24, 970 (1981).

[47] A. Dhar, G. Mandal, S. R. Wadia, "String field theory of two-dimensional QCD: A Realization of W(infinity) algebra," Phys. Lett. B329, 15-26 (1994). hepth/9403050].

[48] S. R. Das, A. Dhar, G. Mandal, S. R. Wadia, "Gauge theory formulation of the $\mathrm{C}=1$ matrix model: Symmetries and discrete states," Int. J. Mod. Phys. A7, 5165-5192 (1992). hep-th/9110021.

[49] A. Dhar, G. Mandal, S. R. Wadia, "Nonrelativistic fermions, coadjoint orbits of W(infinity) and string field theory at c $=1$," Mod. Phys. Lett. A7, 3129-3146 (1992). [hep-th/9207011].

[50] R. Gopakumar, S. Minwalla, A. Strominger, "Noncommutative solitons," JHEP 0005, 020 (2000). hep-th/0003160.

[51] S. R. Das, A. Jevicki, "Large N collective fields and holography," Phys. Rev. D68, 044011 (2003). hep-th/0304093.

[52] R. d. M. Koch, A. Jevicki, K. Jin, J. P. Rodrigues, " $A d S_{4} / C F T_{3}$ Construction from Collective Fields," Phys. Rev. D83, 025006 (2011). arXiv:1008.0633 [hep-th]].

[53] A. Jevicki, K. Jin, Q. Ye, "Collective Dipole Model of AdS/CFT and Higher Spin Gravity," arXiv:1106.3983 [hep-th]].

[54] F. Lenz, M. Thies, K. Yazaki, S. Levit, "Hamiltonian formulation of twodimensional gauge theories on the light cone," Annals Phys. 208, 1-89 (1991).

[55] O. Aharony, A. Hashimoto, S. Hirano and P. Ouyang, "D-brane Charges in Gravitational Duals of 2+1 Dimensional Gauge Theories and Duality Cascades," JHEP 1001, 072 (2010) arXiv:0906.2390 [hep-th]].

[56] V. Pestun, "Localization of gauge theory on a four-sphere and supersymmetric Wilson loops," arXiv:0712.2824 [hep-th]].

[57] A. Kapustin, B. Willett, I. Yaakov, "Exact Results for Wilson Loops in Superconformal Chern-Simons Theories with Matter," JHEP 1003, 089 (2010). arXiv:0909.4559 [hep-th]]. 
[58] N. Drukker, M. Marino, P. Putrov, "From weak to strong coupling in ABJM theory," Commun. Math. Phys. 306, 511-563 (2011). [arXiv:1007.3837 [hep-th]].

[59] D. L. Jafferis, "The Exact Superconformal R-Symmetry Extremizes Z," arXiv:1012.3210 [hep-th]]

[60] O. Aharony, S. Minwalla and T. Wiseman, "Plasma-balls in large N gauge theories and localized black holes," Class. Quant. Grav. 23, 2171 (2006) arXiv:hepth/0507219].

[61] S. Minwalla, P. Narayan, T. Sharma, V. Umesh and X. Yin, "Supersymmetric States in Large N Chern-Simons-Matter Theories," arXiv:1104.0680 [hep-th].

[62] E. Witten, "SL $(2, Z)$ action on three-dimensional conformal field theories with Abelian symmetry," In *Shifman, M. (ed.) et al.: From fields to strings, vol. 2* 1173-1200 [hep-th/0307041]. 